\title{
DEVELOPMENT OF A REMOTE SENSING BASED CONIFEROUS REGENERATION MONITORING SYSTEM
}

\author{
By \\ Darren Pouliot, HBScF \\ A thesis submitted to \\ The Faculty of Graduate Studies and Research \\ in partial fulfillment of \\ the requirements for the degree of
}

\section{Doctor of Philosophy}

Department of Geography and Environmental Studies

Carleton University

Ottawa, Ontario

(C) May, 2006, Darren Pouliot 


$\begin{array}{ll}\begin{array}{l}\text { Library and } \\ \text { Archives Canada }\end{array} & \begin{array}{l}\text { Bibliothèque et } \\ \text { Archives Canada }\end{array} \\ \begin{array}{l}\text { Published Heritage } \\ \text { Branch }\end{array} & \begin{array}{l}\text { Direction du } \\ \text { Patrimoine de l'édition }\end{array} \\ \begin{array}{l}\text { 395 Wellington Street } \\ \text { Ottawa ON K1A ON4 }\end{array} & \begin{array}{l}\text { 395, rue Wellington } \\ \text { Ottawa ON K1A ON4 } \\ \text { Canada }\end{array}\end{array}$

Your file Votre référence ISBN: 978-0-494-18231-4 Our file Notre référence ISBN: 978-0-494-18231-4

NOTICE:

The author has granted a nonexclusive license allowing Library and Archives Canada to reproduce, publish, archive, preserve, conserve, communicate to the public by telecommunication or on the Internet, loan, distribute and sell theses worldwide, for commercial or noncommercial purposes, in microform, paper, electronic and/or any other formats.

The author retains copyright ownership and moral rights in this thesis. Neither the thesis nor substantial extracts from it may be printed or otherwise reproduced without the author's permission.
AVIS:

L'auteur a accordé une licence non exclusive permettant à la Bibliothèque et Archives Canada de reproduire, publier, archiver, sauvegarder, conserver, transmettre au public par télécommunication ou par l'Internet, prêter, distribuer et vendre des thèses partout dans le monde, à des fins commerciales ou autres, sur support microforme, papier, électronique et/ou autres formats.

L'auteur conserve la propriété du droit d'auteur et des droits moraux qui protège cette thèse. $\mathrm{Ni}$ la thèse ni des extraits substantiels de celle-ci ne doivent être imprimés ou autrement reproduits sans son autorisation.
In compliance with the Canadian

Privacy Act some supporting forms may have been removed from this thesis.

While these forms may be included in the document page count, their removal does not represent any loss of content from the thesis.
Conformément à la loi canadienne sur la protection de la vie privée, quelques formulaires secondaires ont été enlevés de cette thèse.

Bien que ces formulaires aient inclus dans la pagination, il n'y aura aucun contenu manquant.

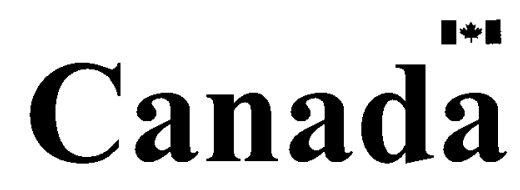




\begin{abstract}
Sustainable forest management depends on timely regeneration of disturbed forests. During the regenerating stage many factors can substantially alter the composition and structure of the developing forest. Monitoring at this time is critical to ensure that desirable forests are established and provide for future needs. This research evaluates the potential of digital remote sensing for monitoring regeneration. Automated methods for detecting and delineating individual conifer crowns and for estimating the abundance of woody deciduous competing vegetation are developed using leaf-off imagery (i.e. when leaves have not flushed). Detection and delineation were tested on several sites with different regeneration characteristics. Results show that errors less than $20 \%$ can be achieved if viewing geometry is controlled (view angles $<15^{\circ}$ ) and the minimum tree size (i.e. crown diameter) to be detected is greater than $30 \mathrm{~cm}$ with $\sim 6 \mathrm{~cm}$ resolution imagery. Modeling of deciduous competition abundance showed that a useful relation existed between extracted image features and measurements made in the field. With the methods developed, a automated processing system for regeneration monitoring is possible.
\end{abstract}




\section{ACKNOWLEDGEMENTS}

There are many people and organizations that have supported me in this work. Research and personnel funding was provided by the National Sciences and Engineering Research Council. Other contributions for the research came from the Ontario Ministry of Natural Resources, the Canadian Forest Service, Wiskair Ltd., Duncantech Ltd. (now Redklake Inc.), and Buchanan Forest Products Ltd.

I have been fortunate to have had the opportunity to work with an excellent supervisor Dr. Doug King who recognized my potential and provided opportunities, encouragement, knowledge, and friendship. I hope that if I find myself in a similar situation I can provide such exceptional guidance. It has also been a privilege to have had access to Dr. Doug Pitt and Wayne Bell who helped with the research focus, design, analysis and provided keen insight into current forest management issues and practices. I would also like to mention the efforts of Mark Lindsay, who aided in the field work for this research.

Finally, this undertaking has been a considerable challenge and I'd like to extend my gratitude to my family Don, Eleanor, Chris, Andrea, and Andrew who have each helped me in their own ways. I'd especially like to thank to my wife Tracey whose encouragement and support was greatly needed and appreciated. 


\section{TABLE OF CONTENTS}

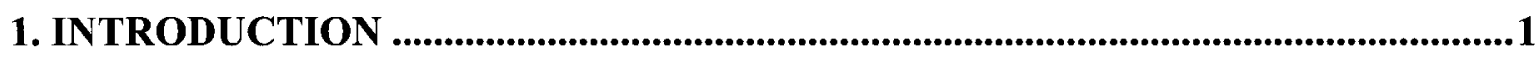

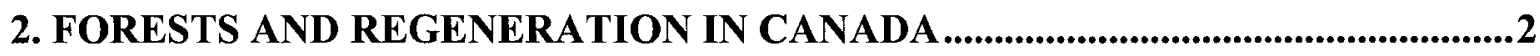

2.1 Distribution OF Forest TyPES AND STRUCTURE............................................

2.2 ECONOMIC, SOCIETAL AND ECOLOGICAL FOREST VALUES ...................................... 4

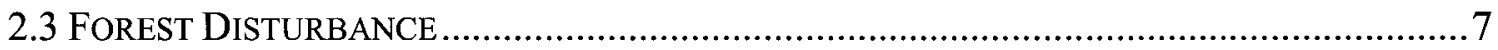

2.4 FOREST REGENERATION.................................................................................

2.4.1 Factors Limiting Regeneration Establishment .............................................. 9

2.4.2 Controlling Competing Vegetation ............................................................. 10

3. REGENERATION ASSESSMENT .....................................................................13

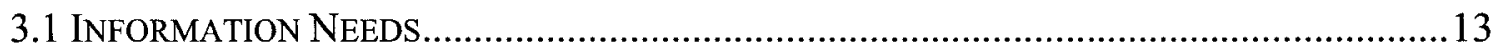

3.2 APPROACHES TO REGENERATION ASSESSMENT .................................................. 14

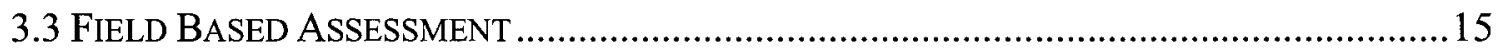

3.4 POTENTIAL FOR REMOTE SENSING IN REGENERATION ASSESSMENT ........................16

3.4.1 Justification of a Remote Sensing Based Approach........................................... 16

3.4.2 Spectral Properties of Remote Sensing Imagery .......................................... 17

3.4.3 Spatial Properties of Remote Sensing Imagery ...............................................20

3.4.4 Air-Photo Interpretation for Regeneration Assessment....................................22

3.4.5 Medium Resolution Approaches to Regeneration Assessment ...........................25

3.4.6 High Resolution Approaches to Regeneration Assessment ...............................26

4. OVERVIEW OF RESEARCH TOWARDS THE DEVELOPMENT OF A REMOTE SENSING BASED REGENERATION MONITORING SYSTEM...........27

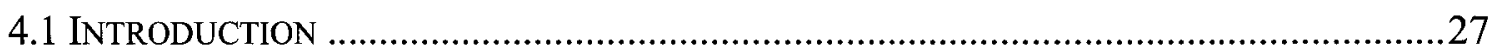

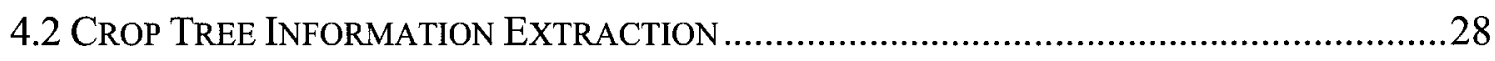

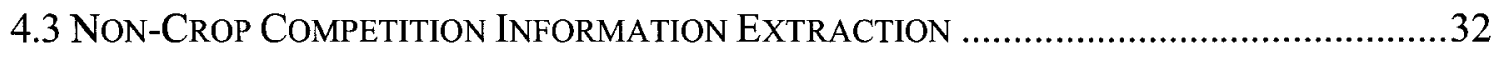

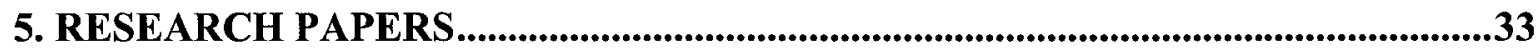

5.1 Development and Evaluation of an Automated Tree Detection ANd DELINEATION ALGORITHM IN CONTROLLED EXPERIMENTAL CONDITIONS ......................33

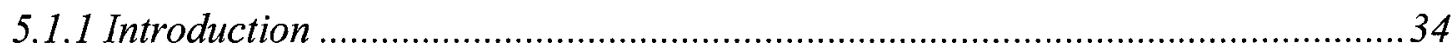

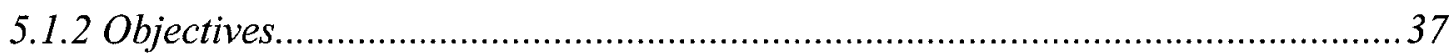

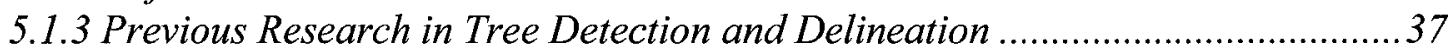

5.1.3.1 Tree Detection and Delineation Algorithms ..............................................37

5.1.3.2 Tree Detection and Delineation Accuracy Assessment ...............................39

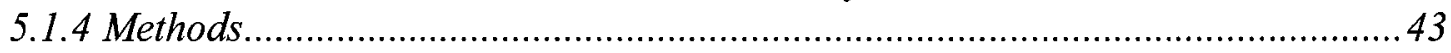

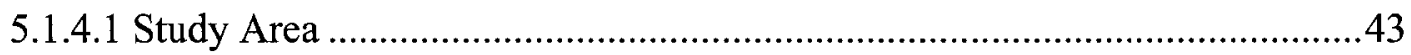

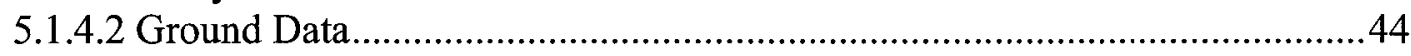

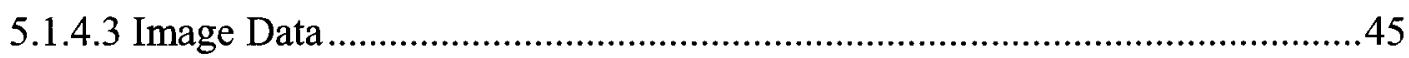

5.1.4.4 Tree Detection and Delineation Algorithm .........................................45

5.1.4.5 Detection and Delineation Algorithm Evaluation ....................................53

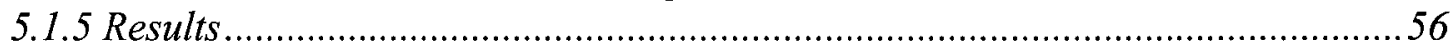

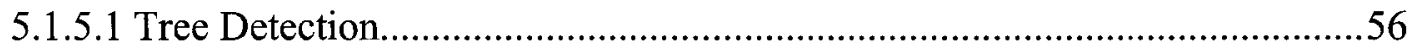

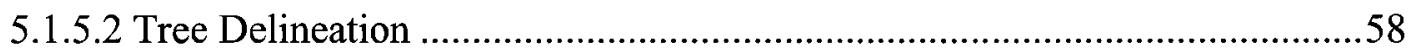




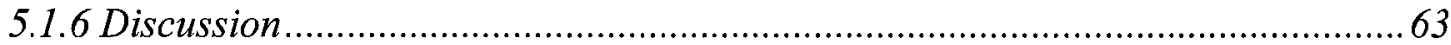

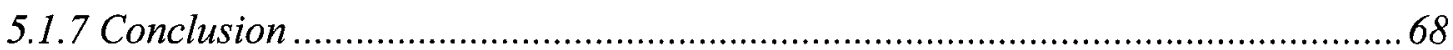

5.2 Determing AN OPtimal APPROACH FOR Automated Tree DeteCtion .................69

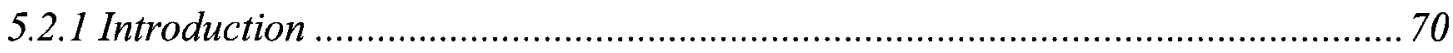

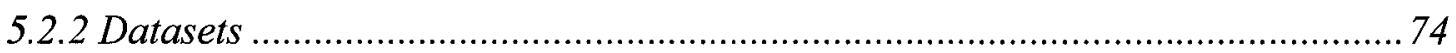

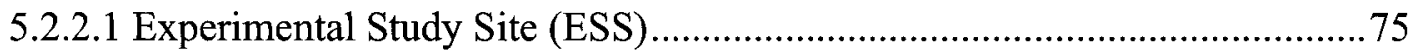

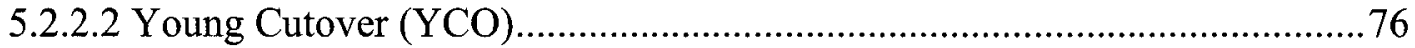

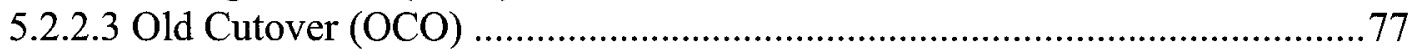

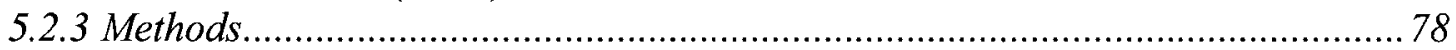

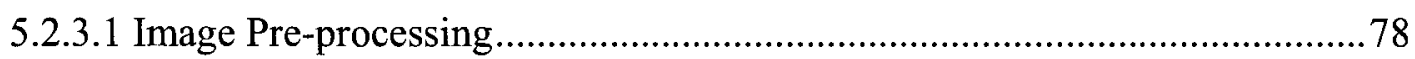

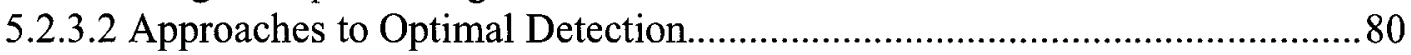

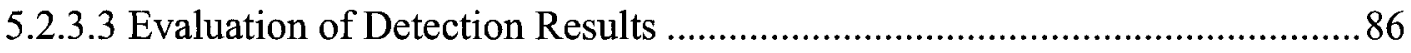

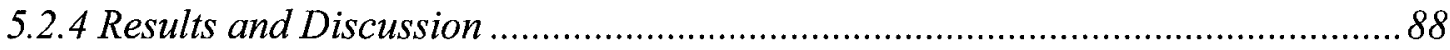

5.2.4.1 Empirically Derived Optimal Smoothing ............................................ 88

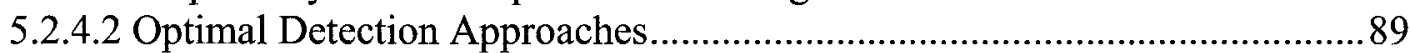

5.2.4.3 Comparison with Other Research ........................................................96

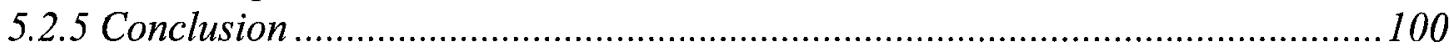

5.3 DeVElopment and EVAluation of AN Automated Tree Detection-Delineation ALGORITHM FOR OPERATIONAL MONITORING OF REGENERATING CONIFEROUS FORESTS

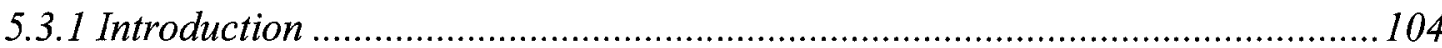

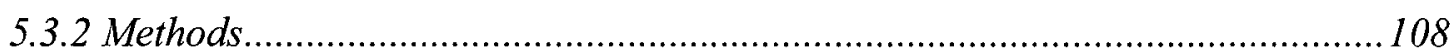

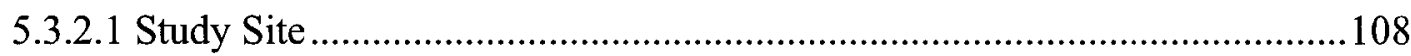

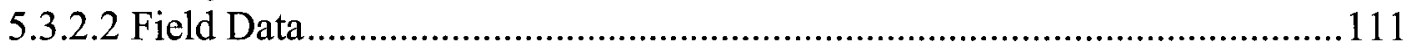

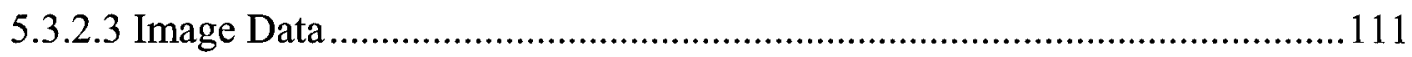

5.3.2.4 Tree Detection-Delineation Algorithm ................................................. 112

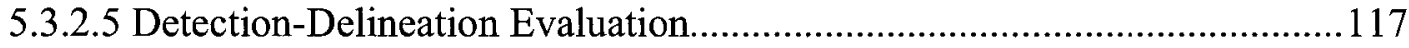

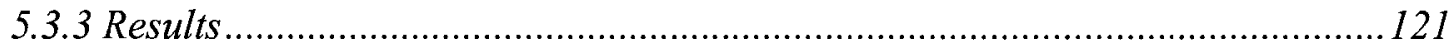

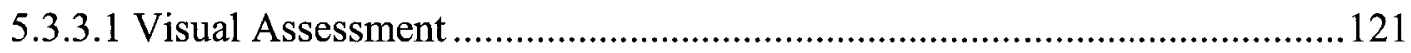

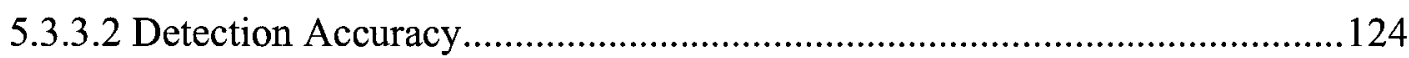

5.3.3.3 Delineation Accuracy ........................................................................... 128

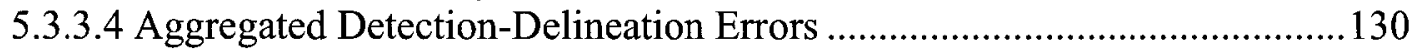

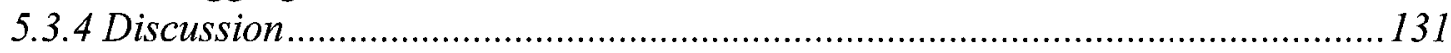

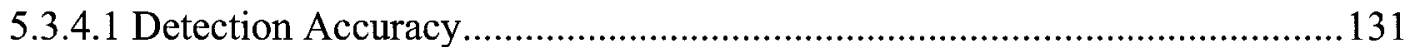

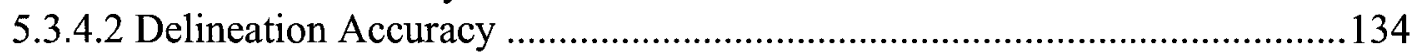

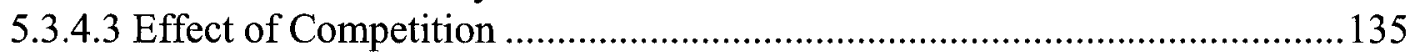

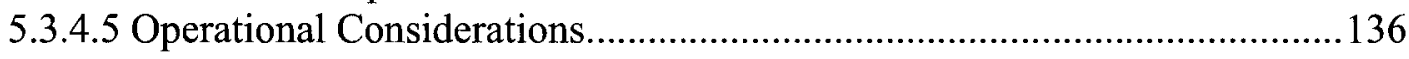

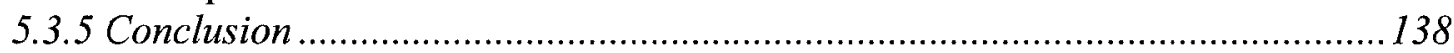

5.4 Large Area Operational EVAluation of Automated Tree Detection AND

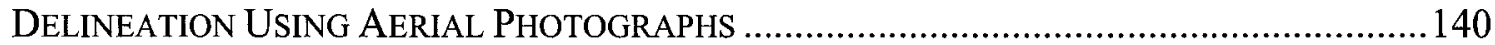

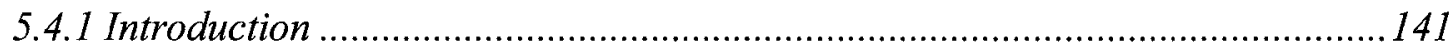

5.4.1.1 Automated Tree Detection and Delineation ............................................ 142

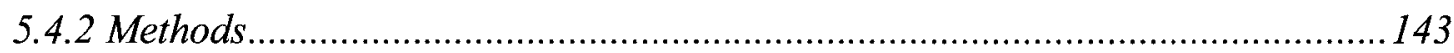

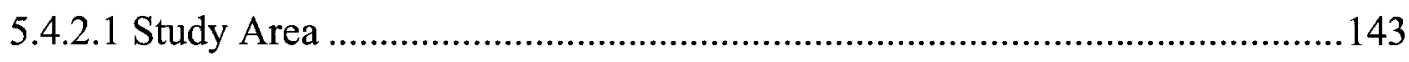

5.4.2.2 Leaf-Off Aerial Photography ............................................................. 144

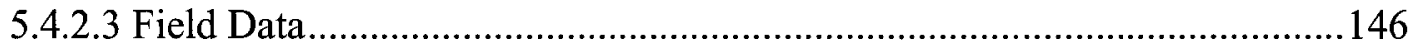


5.4.2.4 Image-Based Conifer Detection-Delineation and Measurement 147

5.4.2.5 Detection and Delineation Accuracy Evaluation........................................ 148

5.4.3 Results ................................................................................................ 149

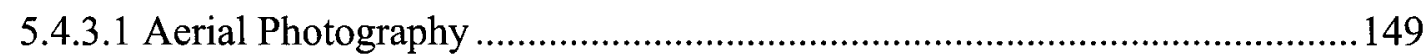

5.4.3.2 Automated Conifer Detection Results .................................................. 149

5.4.3.3 Automated Conifer Crown Delineation Results ........................................ 153

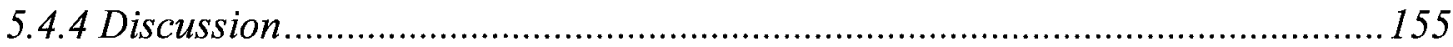

5.5 DEVELOPMENT AND EVALUATION OF AN AUTOMATED APPROACH FOR NON-CROP COMPETITION ASSESSMENT USING LEAF-OFF IMAGERY .............................................. 159

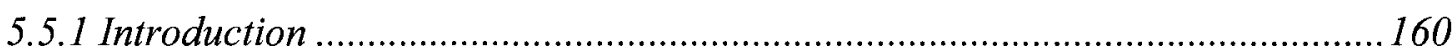

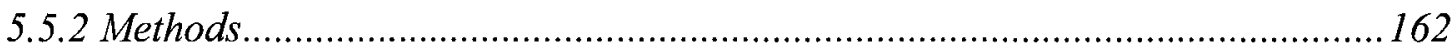

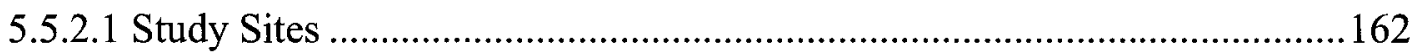

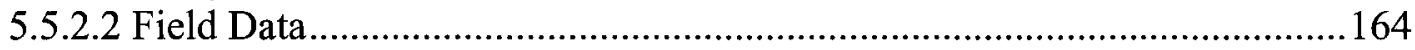

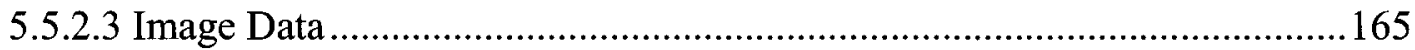

5.5.2.4 Distinctive Image Properties Linked to Competition Abundance...............165

5.5.2.5 Extraction of Hardwood and Shrub Competition Abundance Measures.... 167

5.5.2.6 Modeling Hardwood and Shrub Competition Abundance ........................175

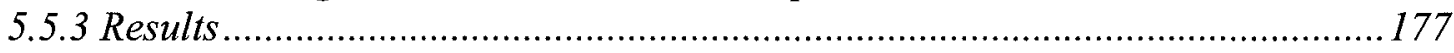

5.5.3.1. Extraction of Hardwood and Shrub Abundance Measures....................... 177

5.5.3.2 Modeling Hardwood and Shrub Competition Abundance .......................... 181

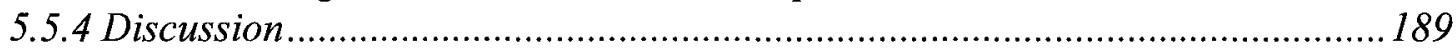

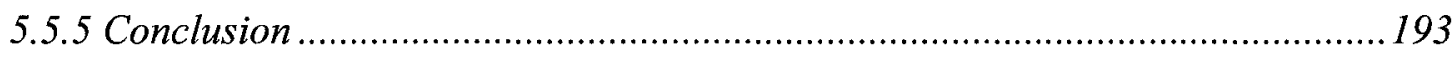

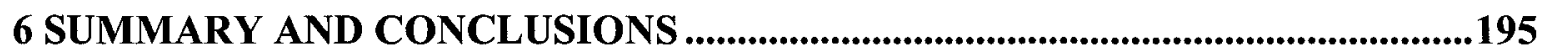

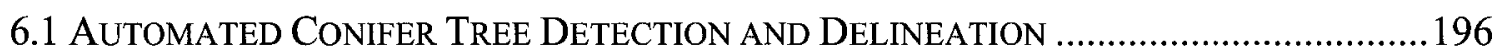

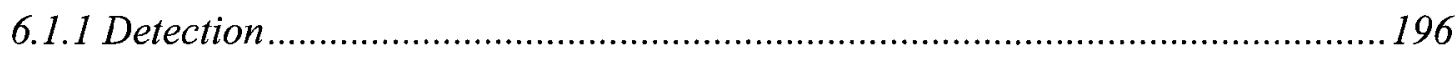

6.1.2 Delineation.................................................................................................. 198

6.1.2 Effects of Imaging Conditions on Detection and Delineation ..........................199

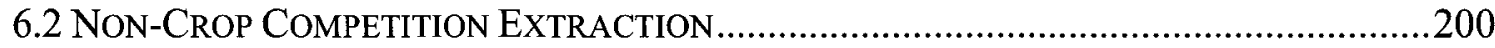

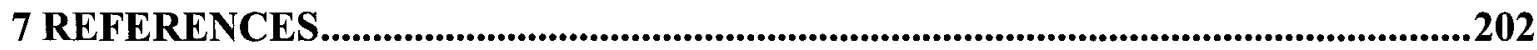




\section{LIST OF TABLES}

Table 5.1.1: Results of tree detection for several ordinary local maximum filters with fixed window sizes $(15 \times 15$ to $30 \times 30$ pixels $)$ and for the LMRDA at each of the pixel spacings studied $(5$ to $30 \mathrm{~cm})$.

Table 5.1.2: Error in average crown diameter measured from automated delineations at four image pixel spacings and for manual delineation at the $5 \mathrm{~cm}$ pixel spacing 59

Table 5.1.3: Delineation accuracy for crown diameter range of $30-100 \mathrm{~cm}$ with outliers caused by short ground vegetation removed................................................................62

Table 5.2.1: Summary of selected characteristics for datasets used in the study. ................75

Table 5.2.2: Empirically derived tree detection accuracy for different Gaussian smoothing factors.

Table 5.2.3: Detection error and accuracy for the four optimal detection approaches........90

Table 5.3.1: Detection accuracy with increasing Gaussian smoothing intensity

Table 5.3.2: Detection accuracy for all trees and for trees with crown diameters greater than 30 and $60 \mathrm{~cm}$

Table 5.3.3: Counts of detection error category and source ...........................................127

Table 5.3.4: Summary statistics for delineation accuracy ................................................128

Table 5.3.5: Transect mean and percent difference measures between image and field measured tree density, crown diameter, and height...................................................................131

Table 5.4.1: Comparison of field and automated tree counts per plot...............................150

Table 5.4.2: Comparison of the number of trees counted in each plot for field, automated

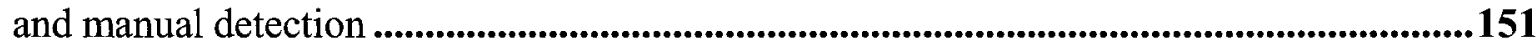

Table 5.4.3: Automated tree count error (\# trees) for each view angle category ...............152

Table 5.4.4: Comparison of the average crown diameter $(\mathrm{cm})$ measured in the field to that from automated crown delineation.

Table 5.4.5: Comparison of average crown diameter measured using the three methods for a subset of 21 of 44 randomly selected plots: field measurement, measurement from automated delineations, and measurement from manual delineations. ................................154

Table 5.4.6: Delineation error $(\mathrm{cm})$ for each view angle category.................................154

Table 5.5.1: Classification accuracy for selected feature combinations.............................181

Table 5.5.2: Model results for the competition index (CI) measured in meters. ................186

Table 5.5.3: Model results for competition stem density $\left(\right.$ stems $\mathrm{ha}^{-1}$ )...............................188 


\section{LIST OF FIGURES}

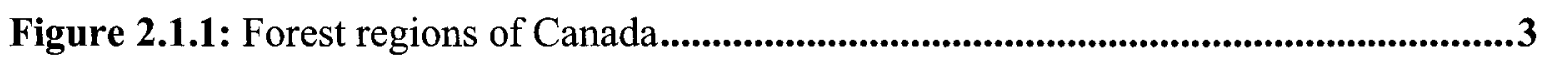

Figure 2.3.1: Annual forest area impacted by insects, fire, and harvesting..........................7

Figure 3.2.1: Conceptual diagram of key characteristics of several approaches to regeneration assessment......................................................................................................................15

Figure 3.4.1: Spectral curves for common land cover features .........................................18

Figure 5.1.1: Three dimensional view of large and small tree crowns in the near infrared band for pixel sizes of $5 \mathrm{~cm}$ and $15 \mathrm{~cm}$................................................................42

Figure 5.1.2: Histogram showing the distribution of field measured crown diameters.... .45

Figure 5.1.3: Overview of tree detection algorithm (LMRDA) process ...............................47

Figure 5.1.4: Example images showing image pre-processing for optimal detection and delineation.......................................................................................................................47

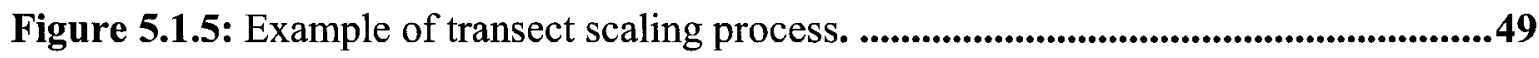

Figure 5.1.6: Effect of removing points that cause extreme internal polygon angles in delineated crowns. .......................................................................................................................53

Figure 5.1.7: Example images showing the results of resampling to pixel spacings of 10 $\mathrm{cm}, 15 \mathrm{~cm}$, and $30 \mathrm{~cm}$ from the original $5 \mathrm{~cm}$ pixel spacing.................................................56

Figure 5.1.8: Detection results using $5 \mathrm{~cm}$ pixel spacing imagery ...................................58

Figure 5.1.9: The relation between absolute error in crown diameter measured from LMRDA delineated crowns and ground measured crown diameter.................................60

Figure 5.1.10: Example delineation results of LMRDA and the manual method using $5 \mathrm{~cm}$

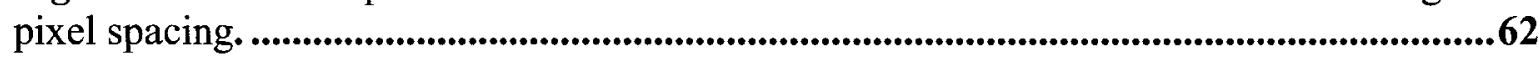

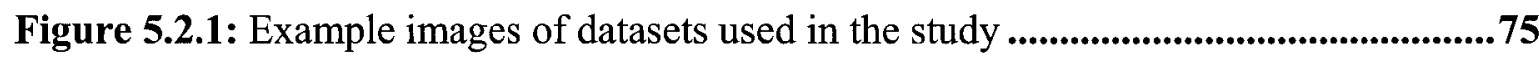

Figure 5.2.2: Example local maxima smoothing relation......................................................881

Figure 5.2.3: Example of original image, smoothing factor image, and locally smoothed

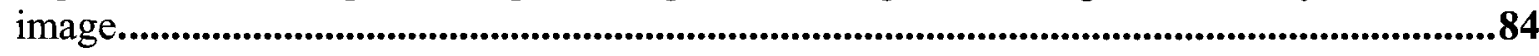

Figure 5.2.4. Relation between mean local edge estimate for a crown and degree of Gaussian smoothing required to detect only one local maximum for the crown object.......85

Figure 5.2.5: Diagram of post detection merging algorithm approach...............................86

Figure 5.2.6: Example detection results for the three datasets, reference tree locations, and four detection methods compared............................................................................................91

Figure 5.2.7: Local maxima smoothing relations for the three datasets used in the study ..92

Figure 5.3.1: Study site locations. ....................................................................................109

Figure 5.3.2: Flow chart showing stages involved in algorithm implementation ..............112

Figure 5.3.3: Example local maxima smoothing relation................................................115 
Figure 5.3.4: Example detection-delineation results for selected plots..

Figure 5.3.5: Example overview images of detection-delineation results for the three

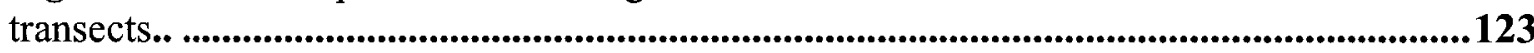

Figure 5.3.6: Examples of detection error sources ......................................................126

Figure 5.3.7: Scatterplots of field measured vs. image measured average crown diameter for trees matched as one automated segment to one reference ellipse 129

Figure 5.3.8: Scatterplots of the difference between image and field measured mean plot crown diameter and the stem density of woody competition.

Figure 5.4.1: Example ground conditions.

Figure 5.4.2: An area imaged at view angles of 15, 24, and 33 degrees in different photos.

Figure 5.4.3: Example aerial photograph. ......................................................................150

Figure 5.4.4: Automated tree detection error for sampled view angles..............................152

Figure 5.4.5: Mosaic of block 4 showing bounds of the $0-10,0-15$, and $0-20^{\circ}$ view angles.

Figure 5.4.6: Overlay of automated delineation results on image data.

Figure 5.4.7: Error in average crown diameter measured from automated crown delineations.

Figure 5.5.1: Example ground conditions at each site.

Figure 5.5.2. Characteristics of competition in leaf-off imagery.

Figure 5.5.3: Hardwood and shrub class separability results for the original RGB bands with an additional texture measure 180

Figure 5.5.4: Example results of hardwood stem detection 182

Figure 5.5.5: Scatterplot of manual and automated standardized total stem length of hardwood stems per plot

Figure 5.5.6: Scatterplot of manual and automated fuzzy pixel counts per plot for the shrub class.

Figure 5.5.7: Scatterplots of hardwood and shrub competition indices estimated from imagery using manual and automated methods versus the field measured competition index for competition $>0.5 \mathrm{~m}$ in height

Figure 5.5.8: Scatterplots of hardwood and shrub competition stem density estimated from imagery using manual and automated methods versus field measured competition stem density for competition $>1 \mathrm{~m}$ in height 


\section{INTRODUCTION}

The focus of this research is directed towards the development of an automated approach to regeneration monitoring using high-resolution airborne remote sensing. Monitoring regeneration is critical for sustainable forest management to ensure that desired and productive forest ecosystems are established on disturbed areas. During the establishment stage, many factors can affect the resulting forest structure and the time that is required for forest development. Through timely monitoring, the need for intervention can be identified and an up to date record of forest conditions can be compiled and evaluated in regards to wood supply, wildlife habitat requirements, and other societal needs. A remote sensing approach was taken because of the potential for development of low cost monitoring capabilities that allow for increased spatial and temporal coverage in monitoring efforts. Leaf-off image data is used (i.e. when deciduous vegetation is not green) for conifer crop tree assessment and woody deciduous competition assessment. This allows a single imaging campaign to collect the required data for assessment and is critical for the operational feasibility of a remote sensing based monitoring system.

The thesis is structured as a set of five research papers with additional background information on forests, regeneration, regeneration monitoring and the potential of remote sensing for regeneration monitoring in Canada (Chapters 2 and 3). Specific background information relevant to each paper is not duplicated in this section. A research overview

section (Chapter 4) follows that describes the general objectives and the main findings of each paper. Chapter 5 is comprised of the research papers and includes four published journal articles and a technical report produced for the Ontario Ministry of Natural 
Resources. Four papers are directed towards conifer crop tree assessment using automated image processing techniques, specifically tree crown detection and delineation (sections 5.1-5.4). For competition assessment, a single paper evaluates the potential of leaf-off image data using line detection, classification and modeling (Section 5.5). Chapter 6 serves as a summary and conclusion and was adapted from Pouliot and King (2005b). References have been removed from the papers and compiled into a single reference section in Chapter 7.

\section{FORESTS AND REGENERATION IN CANADA}

\subsection{DISTRIBUTION OF FOREST TYPES AND STRUCTURE}

Forests cover 417.6 million hectares (ha) of Canada's 921.5 million ha landmass and account for $10 \%$ of forests globally. Conifer dominated forests are the most abundant, accounting for $67 \%$ of forest area, followed by mixed coniferous and deciduous forests at $18 \%$, and deciduous dominated forests at $15 \%$ (Natural Resources Canada, 2004). The structure and composition of forests in Canada are most strongly influenced by the north-south climate gradient. The largest trees, thickest canopy cover, and highest component of deciduous tree species occurs in the south and becomes increasing smaller, less thick, and less deciduous dominated towards the north (Archibald, 1995). However, at finer scales forest structure and composition is largely defined by local climate, soil properties, topography, and site history (Smith et al., 1997).

There are several stratification schemes used to describe the distribution of forests in Canada. Rowe (1972) defines 10 regions based on dominant tree species and physical 
characteristics of the forest that are appropriate for developing an understanding of the distribution of major forest types (Figure 2.1.1).

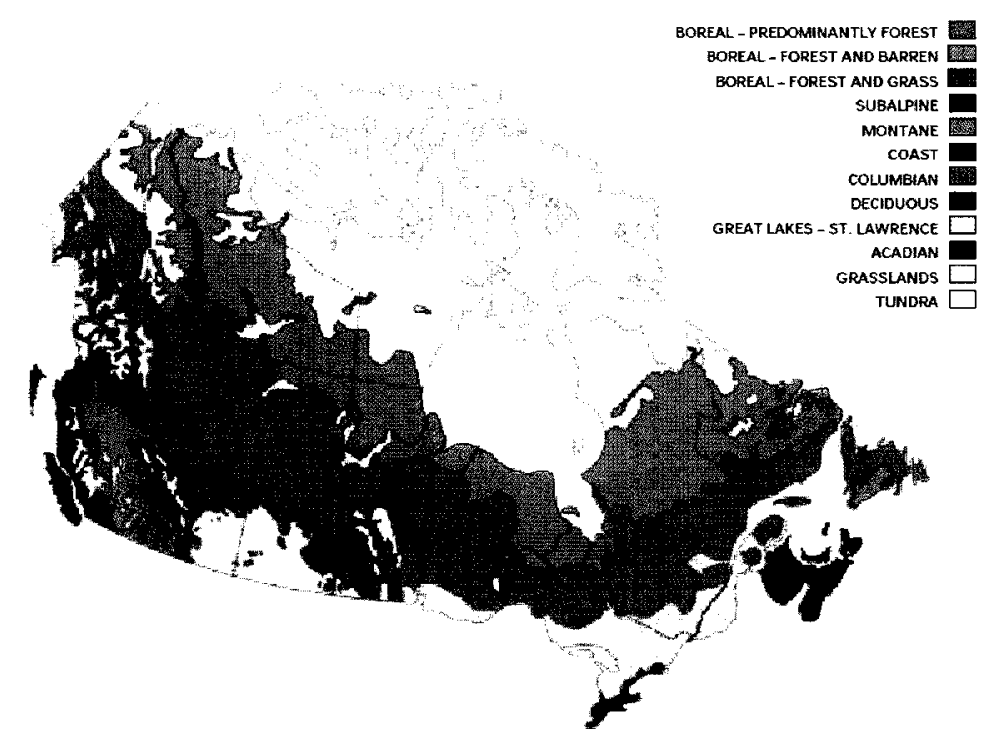

Figure 2.1.1: Forest regions of Canada from Farrar (1995).

The boreal forest is the largest of the forest regions dominated by coniferous species; white spruce (Picea glauca), black spruce (Picea mariana), and tamarack (Larix Laricina; Farrar, 1995). Black spruce becomes more abundant towards the north marked by small or stunted tree growth and lower tree densities. In this area trees greater than 300 years of age often only reach heights of 2-3 meters (Larsen, 1980). To the west, pure stands of lodgepole pine (Pinus contorta) are found, whereas in Central and Eastern Canada, lodgepole pine is replaced by jack pine (Pinus banksiana; Archibold, 1995). To the south, in Central Canada, boreal deciduous species trembling aspen (Populus tremuloides), balsam poplar (Populus balsamifera) and willow (Salix spp.) become increasingly abundant (Farrar, 1995). 
Deciduous dominated forest regions include the Deciduous, Great Lakes St. Lawrence, and the Acadian. These regions are closely related with principal species consisting of sugar maple (Acer saccharum), white oak (Quercus alba), white birch (Betula papyrifera), yellow birch (Betula alleghaniensis), eastern hemlock (Tsuga canadensis), white pine (Pinus strobus), and red pine (Pinus resinosa). In addition to these deciduous species, other characteristic species of the Deciduous region include hickory (Carya spp.), black walnut (Juglans nigra), and American beech (Fagus grandifolia; Farrar, 1995).

In Western Canada, principally British Columbia, four different regions characterize the different forest types resulting from the strong influence of the costal environment and rugged topography, in addition to the north-south climate gradient that is dominant in the rest of Canada. Douglas fir (Pseudotsuga menziesii) is a characteristic species of the Coast, Montane, and Columbian forest regions. In the subalpine region the higher elevation favours species such as Engleman spruce (Picea englemanni) and subalpine fir (Abies Lasiocarpa; Farrar, 1995).

\subsection{ECONOMIC, SOCIETAL AND ECOLOGICAL FOREST VALUES}

Due the vast extent of forests in Canada, forests have become a vital resource to Canada's social, ecological and economic stability. The forest enriches the lives of Canadians and is an important part of the Canadian identity. This is reflected by the incorporation of native tree species into national and provincial symbols such as the maple leaf on the 
national flag. Forest use, management, and sustainability are important issues to Canadians.

Traditionally, the forest has been considered mostly as a source of fiber for the wood products industry. This view of the forest has developed in the past due to the economic gains derived from forest products and the large forest resource (Apsey et al., 2000). Today, the wood products industry contributes significantly to the Canadian economy providing 361,100 direct and an estimated 268,100 indirect forestry jobs (Natural Resources Canada, 2004). In 1999, the forest industry was valued at 44.2 billion dollars in export revenue. It is of particular importance to rural communities who derive much of their employment from the forest industry (Taylor, 1999).

Non-wood based products generate a modest annual revenue of $\$ 241$ million for rural and first nations communities. Products include maple sap, wild mushrooms, wild fruits, medicinal plants, ornamentals and essential oils (Duchesne et al., 2000). Recreation is also substantial. In 1987 hunters spent \$ 139 million to hunt in Ontario. Anglers also spent a substantial amount, \$ 981 million (Ontario Environmental Assessment Board, 1994). Other forest uses such as birding, hiking, biking, and cross-country skiing are difficult to value economically, but are an important part of a satisfactory life for many Canadians. Spiritual and historical forest uses are similar in this respect. Many people visit forests as a means of reducing stress. Getting away from the frustrations of urban life and experiencing nature can be a powerful psychological uplift (Beckley, 1999). 
Historical values provide a sense of place and familiarity with one's surroundings. Examples of historical values include spirit rocks and burial grounds (Grose, 1995).

The ecological services that forests provide typically have no directly measurable economic value, but in many cases are essential to human survival. Forests play a key role in freshwater supplies, by helping to maintain soil permeability allowing for groundwater recharge (Environment Canada, 2004). They prevent erosion by intercepting incoming precipitation and restricting surface water flow (Chang, 2003). Forests sequester carbon in the atmosphere storing it in tree tissues and soil. Estimates for Canada suggest that over the last century forests have been a net sink of atmospheric carbon helping to reduce the human impact on climate change (Kurz and Apps, 1996; Auclair and Bedford, 1997; Chen et al., 2000). Forests are also key contributors to the global supply of oxygen (Kimmins, 1997). They help to maintain biodiversity, enhancing the resilience of forest ecosystems to change (Kimmins, 1997). Additionally, biodiversity is regarded as an important attribute because biologically diverse environments store chemicals and genetic material that may become beneficial to humans as they are discovered (Tokeshi, 1997). Wildlife also depends on plant and ecosystem diversity for survival. The link between suitable habitat and healthy wildlife has long been known to biologists along with the vital role that wildlife play in creating healthy ecosystems (Caza and Neave, 2000). 


\subsection{FOREST DISTURBANCE}

Disturbance in the boreal forest results from either human or natural causes such as harvesting, road building, mining, agricultural expansion, flooding, blowdown, fire, insects and disease. These disturbances result in a significant amount of annual forest removal and change to the forest structure. Under the current national reporting system, only disturbances due to fire, insects and harvesting are made available on an annual basis making it difficult to evaluate the full magnitude of forest disturbance in a given year (Gillis and Leckie, 1996). Figure 2.3.1 shows the area depleted by these disturbances from 1970-2001.
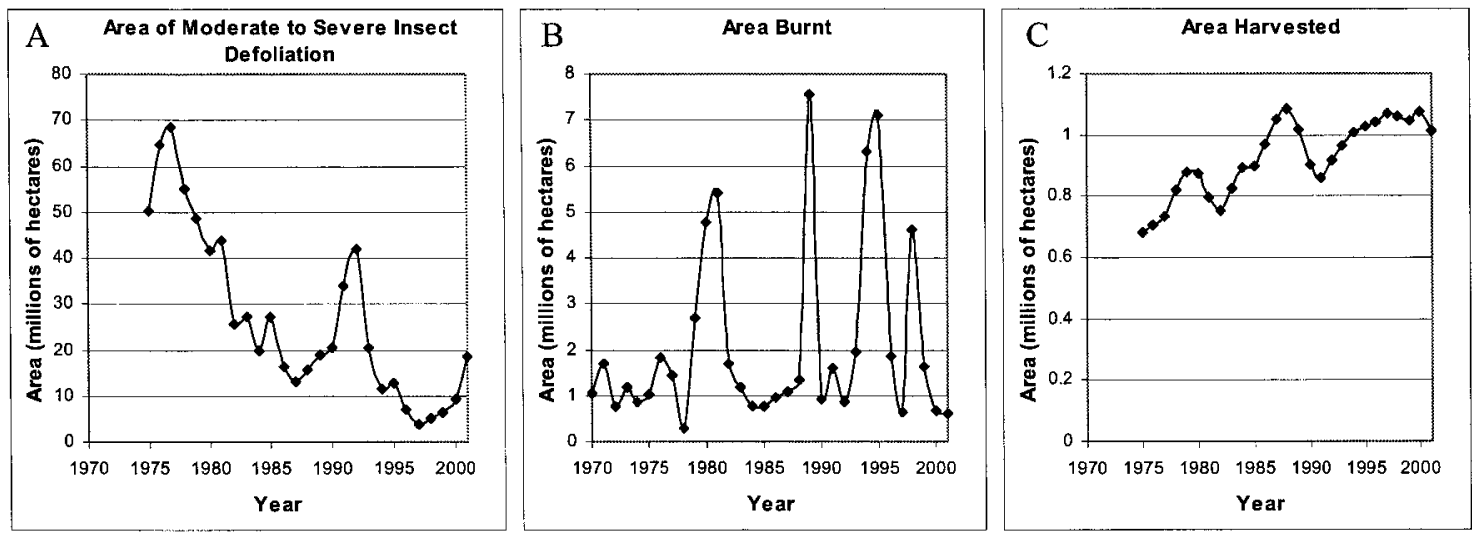

Figure 2.3.1: Annual forest area impacted by insects, fire, and harvesting. Values taken from the National Forestry Database Program (2004). The values reported in Figure 2.3.1A are compiled on an insect by insect basis and, therefore, the same area can be counted more than once exaggerating the total area affected.

Fire and insect damage are the two largest disturbance types in Canada not associated with direct forest removal by humans. Disease damage can also be substantial and can have a greater impact on forest volume growth than insects (Manion, 1991; Callan, 2001). Forest harvesting is the largest direct human disturbance. However, it may not be the most significant in regards to severity. Other forms of human disturbance such as 
road building, mining, and agriculture result in more drastic changes to local vegetation, soil, and hydrology than harvesting. In the boreal forest, $80 \%$ of harvesting is clearcutting. Other harvesting systems based on partial stand removal make up the remainder (Natural Resources Canada, 2004). Forest companies are required by license agreements with provincial governments to reforest harvested areas. Establishment of forests in harvested areas is governed by economic and political factors in addition to ecology. Often re-establishment is focused on developing forests with high wood volume and economically valuable tree species for future wood supplies. However, public concerns to maintain sustainable and diverse forest ecosystems are also taken into consideration.

\subsection{FOREST REGENERATION}

Natural and artificial regeneration are two categories of approaches for forest reestablishment. Natural regeneration involves re-establishing forest cover on depleted lands utilizing local on site resources such as residual trees, seed, and sprouting from roots and stumps. It may often include a modification to the harvest procedure, such as careful logging to maximize the survival of the understory trees, retention of trees as a future seed source or the removal of branches from the tree at the stump to maximize seed dispersal (Smith et al., 1997). However, it is difficult to determine if natural regeneration will produce the desired future forest condition (Greene et al., 1999). For this reason, artificial regeneration is often preferred for areas disturbed by human activity because of greater control (i.e. species and planting/seeding pattern) over the resulting forest structure. Artificial regeneration typically involves planting or seeding treatments to re-establish the new forest (Smith et al., 1997). In Canada, artificial regeneration is 
used to reforest half of the annually harvested area, with the remainder being regenerated by natural means (Natural Resources Canada, 2004).

\subsubsection{Factors Limiting Regeneration Establishment}

The growth of trees established through natural or artificial methods can be substantially limited by both abiotic and biotic factors. Abiotic factors include light, nutrients and moisture availability, as well as temperature requirements. Biotic factors include living organisms such as competing vegetation, insects, disease and wildlife. Considering that the abiotic factors for a site are largely controlled by long term by climate, geology, and history, the major factor that influences the availability of resources at a site in the short term is competing vegetation. Other biotic factors such as insect, disease, and animal damage are typically not as significant as competing vegetation, often only being problematic in local areas (Sullivan et al., 1990; Scarr et al., 2001; Whitney et al., 2001).

Competing vegetation reduces the availability of light, moisture, nutrients, and promotes conditions for biotic damage to desired trees. Competing vegetation in this case is known as inter-specific or non-crop vegetation, because it competes for on-site resources with crop trees (desired tree species to be re-established on a site). Removal of competition greatly increases growth and survival of crop trees (Walstad and Kuch, 1987). Similarly, crop vegetation can compete against itself when densities are high (referred to as intraspecific competition), resulting in a phenomenon known as self-thinning. Self-thinning results in the death of less established crop trees, freeing site resources to those remaining (Nyland, 1996). For shade intolerant tree species, overtopping shrub and hardwood (i.e. 
woody) competition can result in substantial mortality within 2-3 years. Shade tolerant species can survive in overtopped conditions, but growth is drastically reduced (Wagner et al., 2001). Herbaceous or low growing vegetation such as grasses are a particular problem for crop tree survival due to the crushing effect it can have when large amounts lean $\backslash$ fall on crop trees. This occurs annually and is amplified by snow, increasing the load on the tree often causing mortality (Wagner et al., 2001). Herbaceous vegetation also causes significant growth reduction and for some species can be more significant than woody competition. The combined presence of both woody and herbaceous competition has the greatest overall impact (Wagner et al., 2001).

\subsubsection{Controlling Competing Vegetation}

Because vegetative competition can result in substantial mortality and growth reduction, methods to control it have been developed and are an important part of forest reestablishment. Methods can be categorized as chemical, mechanical and biological. Chemical control has been the most widely used because it is the most cost effective and results in a high degree of control (Nyland et al., 1996). However, concerns over the safety of releasing chemicals into the environment has led to public disapproval of chemical treatments. More socially acceptable methods include mechanical removal, grazing, cover crops and natural plant toxins (Wagner and Mclaughlan, 1996).

Mechanical treatments involve cutting, breaking, or girdling (i.e. removing bark around the trunk damaging the phloem) of woody competition stems using hand held tools, motorized saws or larger machines. These treatments are not as effective as chemical 
treatments because of the sprouting and suckering capacity of many competing species, which results in their rapid redevelopment (Nyland, 1996). McDonald and Fiddler (1993) reported improved crop tree diameter gains in Douglas fir and ponderosa pine with chemical treatments over manual treatments during a ten-year period. Multiple manual treatments were also applied and resulted in slight diameter gains compared to the chemical treatment. These small gains do not warrant the use of mechanical treatments when costs are considered. The one time application of chemicals to the entire area was $\$ 100$ US ha $^{-1}$, whereas manually clearing a five foot radius around each crop tree three times in ten years cost approximately $\$ 1700 \mathrm{US} \mathrm{ha}^{-1}$.

Biological control methods have evolved from the need to find an effective and socially acceptable treatment. Biological controls involve using naturally occurring organisms to control competing vegetation. These include introducing insects or disease agents that are highly specialized to the competition species, replacing competing vegetation with a vegetation that is less competitive, and introducing grazing animals such as cows and sheep that feed on the competition species. Chondrostereum purpureum is a fungal agent that has been identified as a potential tool to improve the control of competing vegetation after mechanical competition removal. Formulations of the fungus are applied by spray bottle to the freshly cut competition stumps to prevent vegetative reproduction. Pitt et al. (1999) reported that Chondrostereum purpureum applications showed promising results with speckled alder, but red maple and trembling aspen had a more variable response to treatment. Studies with substitute vegetation have been less successful. Willoughby (1999) reviewed the results of using 19 different ground covers to reduce the competitive 
effects of naturally occurring competition on Douglas fir trees. Almost all of the ground treatments proved difficult to establish and all treatments reduced crop tree growth more than that of the naturally occurring vegetation. Of the possible biological control treatments, animal grazing appears to hold the most potential. Sheep, cattle, goats and geese have all been tested as useful grazers to control competing vegetation. In a study reported by Foster (1998), sheep grazing reduced the cover of fireweed, dandelion, and grass to a greater degree than chemical treatments. However, the cost $\left(\$ 205-\$ 705 \mathrm{ha}^{-1}\right)$, complexity involved in effective sheep grazing, and potential for disease transmission to livestock and wildlife are serious limitations to grazing methods.

It is important to point out that the objective of competition control is not to completely remove this vegetation from the site. Some non-crop vegetation provides several benefits to crop tree growth, as well as meets the requirements for some non-timber forest uses. The benefits of non-crop vegetation include (Walstad and Kuch, 1987):

1. Prevention of soil erosion through root and canopy systems;

2. Uptake and recycle site nutrients;

3. Improved soil physical properties by the incorporation of organic matter;

4. Some non-crop competition fixes nitrogen, making it available to crop trees;

5. Reduction in the effects of temperature and humidity extremes through shading and mulching underlying vegetation;

6. Remove browsing pressure on crop trees, as non-crop vegetation is often preferred. 
Thus, balancing the abundance of crop and non-crop species can allow sites to be maintained in a condition of adequate growth and provide for long term site sustainability by reducing soil erosion, providing forage for animals, and enhancing soil productivity.

\section{REGENERATION ASSESSMENT}

\subsection{INFORMATION NEEDS}

For regeneration monitoring, timely information regarding the abundance, distribution, size and health of crop trees and non-crop competitors is needed to ensure that regenerating forests develop according to defined management objectives and to identify where treatment interventions are required. Potential treatments include: fill planting in areas where crop tree abundance is low, removal of competing vegetation in areas where it is significantly impeding crop tree growth, crop tree removal to optimize growth conditions for residual crop trees, and in some cases, fertilizer applications to improve growth limited by a given nutrient. The exact information requirements will vary depending on compromises between information needs, costs associated with data collection, and other operational constraints. The following information is typically required (Pitt et al., 1997):

\section{1) crop species}
a) stem density measured in stems $\mathrm{ha}^{-1}$;
b) average height to the nearest $0.5 \mathrm{~m}$; 
c) stocking (a measure of density relative to a defined standard) to the nearest $10 \%$ of the well spaced (trees separated by a given minimum distance) distribution of trees;

d) health condition, visually assessed for signs of damage.

\section{2) dominant non-crop species}
a) percent cover to the nearest $10 \%$;
b) average height to the nearest $0.5 \mathrm{~m}$;
c) shrub and tree stocking.

In addition to these data, qualitative observations regarding soil types, topography, water bodies and other notable features relevant to treatment options are also recommended.

\subsection{APPROACHES TO REGENERATION ASSESSMENT}

Various methods have been used to collect the needed information, ranging from visual assessment in the field or from the air, to statistically based field sampling and direct measurement. Figure 3.2.1 provides a conceptual diagram of the relative precision/accuracy and cost/time characteristics of the common approaches. Visually based methods tend to be low cost and can be carried out quickly, but the precision and accuracy may be low. Thus, this type of assessment is only recommended for sites where regeneration success or failure is strongly evident. In conditions where it is more difficult to make such an assessment, field sampling and measurement is required (Ontario Ministry of Natural Resources, 2004). Field sampling methods are costly, but are statistically based and have high precision and accuracy relative to other approaches. In between these are air-photo based approaches, which generally consist of visual 
interpretation and mapping. They are typically more costly to perform than field or airborne visual inspection, provide greater coverage than field samples, but do not provide the same precision/accuracy as sample-based field methods.

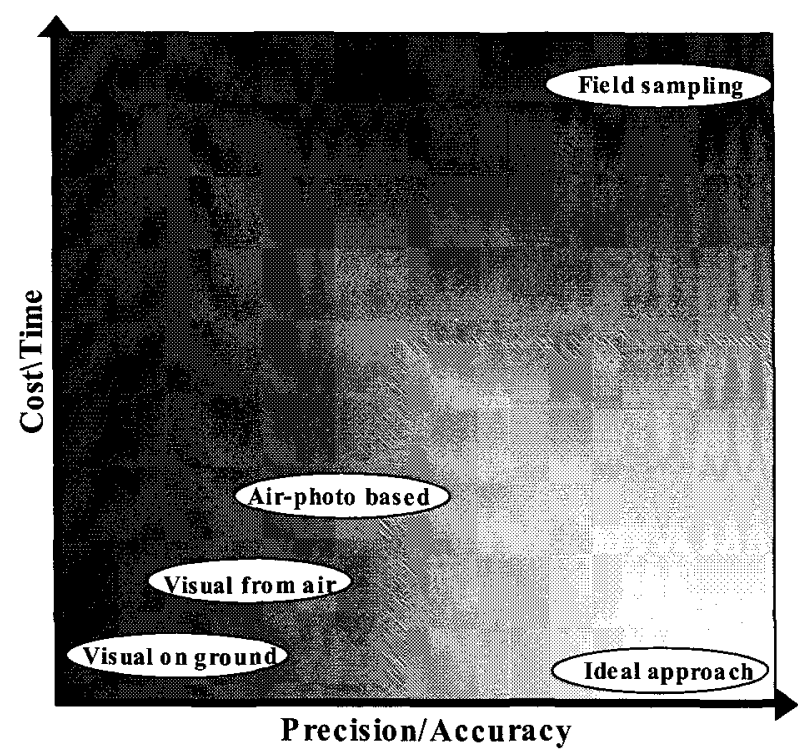

Figure 3.2.1: Conceptual diagram of key characteristics of several approaches to regeneration assessment.

\subsection{FIELD BASED ASSESSMENT}

Specific field sampling assessment procedures have been developed separately by each province to account for differences in regional ecosystem conditions and preferred operational practices. Protocols developed to date include: Silviculture Treatment Assessment and Reporting System for general regeneration monitoring in Ontario (STARS, Pinto et al., 2003); Well-spaced Free-Growing Regeneration Assessment Procedure for Ontario (Ontario Ministry of Natural Resources, 2004); Manitoba Free to Grow Survey Manual (Delaney et. al., 2000); Regeneration Assessment Manual for Saskatchewan (Saskatchewan Environment Forest Service; 2004); Alberta Regeneration Survey Manual (Alberta Sustainable Resource Development, 2003); and the Stocking and 
Free Growing Survey Procedures Manual for British Columbia (British Columbia Forest Practice Branch, 2002). These protocols generally recommend similar approaches using plot based field sampling schemes and free-growing assessment criteria. The typical sampling scheme is a systematic grid with a random start, but other statistically valid designs are acceptable. The timing of assessment is based on the pre-harvest silviculture prescription, but is commonly carried-out within 7-14 years of harvest. Plot sizes vary from $1.72 \mathrm{~m}$ in radius, where only a single tree should be contained and assessed in the plot, to $3.99 \mathrm{~m}$, where several trees should be sampled. Free growing assessment is based on whether a sampled tree is a given distance from another free-growing tree (i.e. well spaced) and is free of a given density of competing vegetation within a certain distance and relative height to the crop tree being assessed. These parameters are defined provincially and can vary by species. The protocols also provide for alternative methods of surveys such as visual or air-photo assessments, but in some cases special permission is required.

\subsection{POTENTIAL FOR REMOTE SENSING IN REGENERATION ASSESSMENT}

\subsubsection{Justification of a Remote Sensing Based Approach}

Remote sensing has several potential advantages over field based methods for regeneration assessment including access to remote areas, development of an image data record that can be used for multiple applications such as analysis of invasive species or woody debris surveys, increased sample area coverage, and low cost of data acquisition per unit area. Of these, the cost advantage is the most critical, as it allows for increased spatial and temporal sampling compared to field data acquisition. To achieve this, 
minimizing the cost of image acquisition is one aspect. Another is the development of image processing methods capable of effectively extracting information from imagery in a reliable and consistent manner that can be implemented by a range of personnel in a timely manner. The following sections review methods that have been developed and evaluated for this purpose.

\subsubsection{Spectral Properties of Remote Sensing Imagery}

Single date imagery consists of both spectral and spatial information. Spectral information refers to the spectral reflectance of surface objects. At the sensor, reflected electromagnetic radiation is broken into classes or bands based on radiometric wavelengths measured in microns $(\mu \mathrm{m})$. The received energy can be partitioned in a multitude of ways based on the sensor design and purpose. For remote sensing of vegetation in the optical region of the spectrum (visible-infrared) useful bands are commonly defined by wavelengths in the blue $(0.4-0.5 \mu \mathrm{m})$, green $(0.5-0.6 \mu \mathrm{m})$, red $(0.6-$ $0.7 \mu \mathrm{m}$ ), near infrared (NIR, 0.7-1.30 $\mu \mathrm{m}$ ), and short-wave infrared (SWIR, 1.3-3.0 $\mu \mathrm{m}$ ). Partitioning the wavelengths in this manner is useful because vegetation absorbs and reflects the radiometric energy at each wavelength differently. In general, vegetation absorbs incoming radiation in the blue and red wavelengths, but reflects a greater proportion in the green, NIR, and SWIR wavelengths. For the visible bands (blue, green and red) this is largely controlled by leaf pigmentation (e.g. chlorophyll). The intensity of the response in the NIR is controlled by the internal structure of the leaf with more heterogeneous cell structures (e.g. more scattering surfaces caused by cell shape, size, layers, and air spaces) increasing reflectance. Plant moisture content is the dominant 
influence on the SWIR band with greater moisture reducing reflectance (Jensen, 1996). The intensity of the response of the target object in each band determines what is known as its spectral signature, which can be used to determine various object properties (Lillesand and Kiefer, 2000). Figure 3.4.1 shows common spectral signatures for typical land cover features.

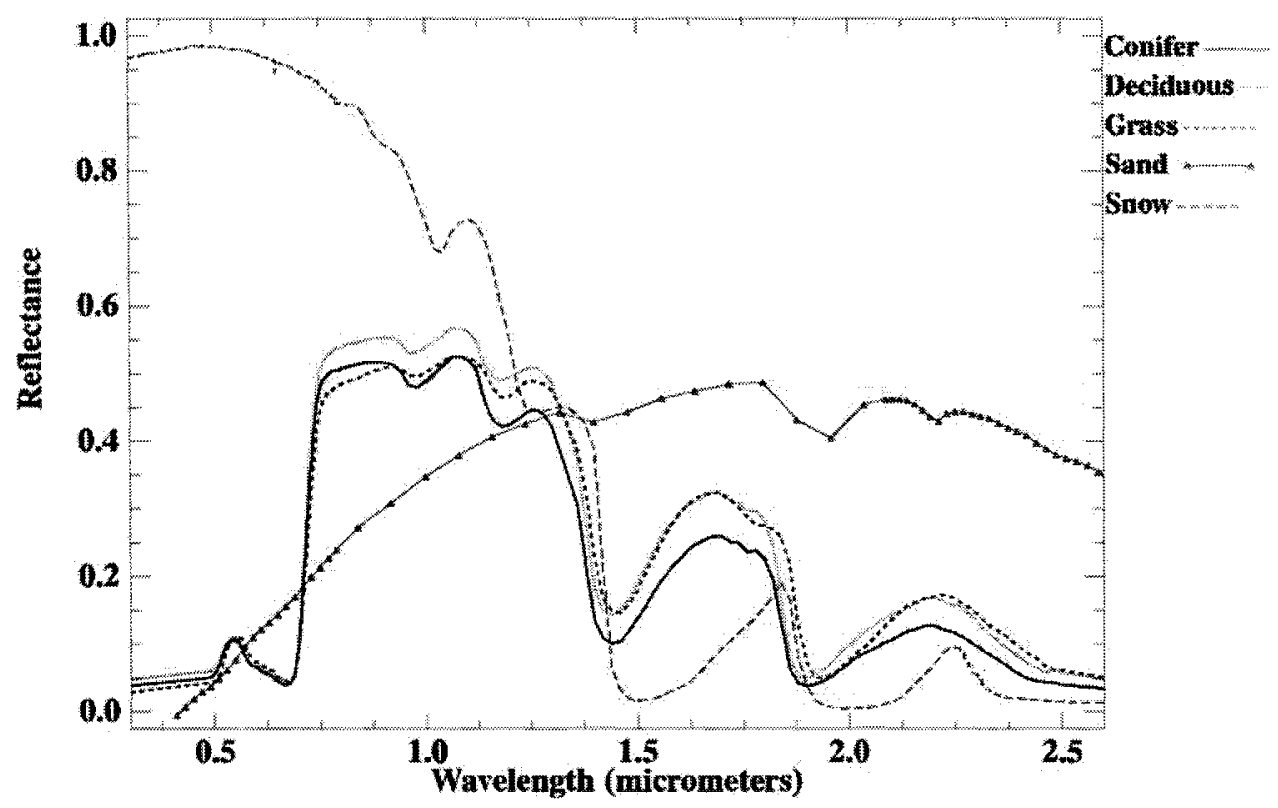

Figure 3.4.1: Spectral curves for common land cover features. Data from Johns Hopkins University, http://speclib.jpl.nasa.gov/.

A large majority of analysis in remote sensing has focused on spectral image properties, such as image classification to map land cover or some other image feature of interest (e.g. Franklin et al, 2002; Latifovic et al., 2004), change detection (e.g. Hall et al., 1991; Mas, 1999; Fraser et al., 2004), biophysical modeling (e.g. Wulder et al., 1996; Chen et al., 2002), and time series analysis to identify trends in surface properties such as vegetation phenology (e.g. Stöckli and Vidale, 2004). 
An important aspect for the success of these applications is minimization of the influence of unwanted variance caused by atmosphere, sun-sensor viewing geometry, sensor error, topography and, in some cases, vegetation phenology on the radiometric properties of the imagery. Methods based on absolute radiometric measurements are most affected, such as in the case of applying a model developed from one image set to another image set at a different time or location. In this case, imagery needs to be well calibrated to a known measurement standard. It typically requires complex algorithms with numerous parameter inputs to characterize the different conditions present when the images were acquired. Often these parameters are not available for a given location or time and must be interpolated spatially and temporally (Jensen, 1996). Methods based on imagery taken under different environmental conditions (i.e. different times) do not necessarily require absolute radiometric measurements, but do require that the image radiometry be relative (i.e. not fixed to a known measurement standard, but have the same response to the same target). For these applications, calibration is much simpler and is typically accomplished by comparing samples from the images and developing correction equations (Du et al., 2005; Olthof et al., 2005). However, in other applications, the sources of unwanted variance can be ignored if their effects on the results are small or the methods are robust to the given error source and magnitude. The methods developed in this research have been designed to require minimal correction, with the majority of parameterization developed from user defined training data selected from image sets collected under the same environmental conditions. 


\subsubsection{Spatial Properties of Remote Sensing Imagery}

Although a measure of success has been obtained with spectral methods, improved and increased information extraction capabilities are possible with the inclusion of spatial information. Visual interpretation relies heavily on the spatial information content in imagery. Spatial properties commonly used by human interpreters include texture, pattern, size, shape, shadow and association. These are briefly described below based on Campbell, (1997):

Texture refers to the variation in image brightness values within a defined area, whereas pattern refers to the arrangement of image objects into distinct recurring forms. However, in the natural world, both pattern and texture combine to create complex textures and, therefore, can be considered to form a general texture category.

Size refers to the dimensions of objects that can be seen in imagery and can be useful in identifying objects with distinct size properties. For example, the width of a road can be used to distinguish between a highway and side road.

Shape is an important property of image features and many features can only be distinguished based on shape. For example, lakes and rivers can only reliably be identified based on shape, as both have similar spectral/textural properties. 
Shadows of objects in an image can be used to help identify the object based on the shape and size characteristics of the shadow silhouette. They can also be important in forming the textural properties of an image.

Association or context is the likelihood of image objects occurring within the same area or within a given proximity. In many cases, knowledge about one object in space can reveal something about the neighbouring objects. For example, forests located along the boundaries of wetlands are most likely composed of moisture tolerant tree species, such as black spruce or tamarack.

In addition to these properties, two others are important in analysis of images. The first is the dependence of some spatial properties on scale. At different scales, the spatial properties discussed above can appear quite different. Thus, in order to effectively measure these properties, the scale of the analysis must first be defined so that it is unique to the object of interest (Lindeberg, 1994). The second is spatial autocorrelation, which refers to the similarity of pixel values with distance. In most images of natural scenes, pixel values will be more similar the closer they are together than further apart in space. Analysis of spatial autocorrelation is useful for determining the scale at which various image features occur and, therefore, can be used to determine the appropriate scale for some types of analysis (Curran, 1988). It is also an important consideration in developing/testing models from remote sensing data where spatial independence of samples is assumed (Diniz-Filho et al., 2003). 
Common spatial based methods used in environmental remote sensing have included various methods to capture image texture, such as simple first order statistical measures within a sample window (Jensen, 1996), co-occurrence matrices (Haralick et al., 1973), image grey level morphology (Laws, 1980), fractals (Emerson et al., 1999) and wavelets (Zhu and Zhang, 1998). These transforms are often combined with spectral information to aid classification or modeling applications. Classification is a form of image segmentation that is typically based on the information associated with an individual pixel. However, another form of image segmentation uses the information associated with groups of pixels in space. Approaches are typically variations of region growing, where pixels are grouped based on similarity measures, or grey-level morphology, such as edge detection and edge following to define object bounds (Gonzalez and Woods, 2002). Once objects are defined, their shape properties and within-object pixel properties can be used to enhance identification. This type of segmentation has been employed for tree crown detection and delineation in mature forests (e.g. Gougeon, 1995a; Brantberg and Walter, 1998; Culvenor; 2000; Persson et. al. 2002, Erikson, 2003). Region-growing segmentation has been incorporated in an iterative classification approach in the commercial software package eCognition (Definiens, 2003). It allows for inclusion of object contextual, textural and shape information in the classification decision.

\subsubsection{Air-Photo Interpretation for Regeneration Assessment}

Visual assessment of air-photos has shown considerable promise for estimating several regeneration attributes. Large-scale photographic (LSP) sampling is one method that can be used to obtain conifer crop tree stocking estimates (Goba, 1982; Smith et al., 1989; 
Hall and Aldred, 1992; Hall, 1994). Instruction manuals for this application have been prepared by Goba et al. (1982) and Hall (1984). In terms of effectiveness, Hall (1984) found a strong relationship between stocking estimates from 1:580 scale photos for jack pine dominated regeneration from 2-6 years of age $\left(\mathrm{r}^{2}=0.82\right.$, standard error $\left.(\mathrm{SE})=8 \%\right)$. For crop trees larger than $30 \mathrm{~cm}$ in height, the relation was improved $\left(\mathrm{r}^{2}=0.961, \mathrm{SE}=5\right.$ \%). Smith et al. (1989) found that photo based counts and ground counts of pine density in 3 to 5 year old plantations were highly related $\left(\mathrm{r}^{2}=0.75, \mathrm{SE}=233\right.$ stems ha $\left.{ }^{-1}\right)$ for 1:4000 to 1:6000 scale photos. Hall and Aldred (1992) found strong relations between photo and ground measured stocking and density estimates using 1:500 scale photographs of 5-23 year conifer regeneration sites (stocking $-r^{2}=0.96, S E=7 \%$; density $-r^{2}=0.98$, $\mathrm{SE}=939$ stems ha $^{-1}$ ). However, they concluded that density could not be reliably determined due to high standard errors caused by small trees or trees within clusters being missed. The potential for species identification was also assessed and produced a high accuracy (96\%) for jack pine and spruce discrimination.

For competition assessment, Smith et al. (1989) found a moderate relation between leafon photo measured percent woody cover and ground measured basal area $\left(\mathrm{r}^{2}=0.42, \mathrm{SE}=\right.$ $\left.5.5 \mathrm{~m}^{2} \mathrm{ha}^{-1}\right)$. Pitt and Glover (1993) found strong relations between 1:414 scale leaf-on photos and ground measured plot average crown areas $\left(r^{2}=0.97\right)$ for several deciduous tree covers. In agreement with other findings, density did not produce a strong relation without discounting smaller trees in the ground based density estimates. In this case, for trees with a crown area greater than $0.1 \mathrm{~m}^{2}$ the relation between photo and field estimated density was high $\left(\mathrm{r}^{2}=0.97\right)$. In another study, with a greater variety of forest conditions, 
measures of individual trees were also made with high accuracy (Pitt and Glover, 1996). Using a two-phase sampling design, where a small sample of ground measures were acquired to calibrate photo estimates, Pitt et al. (1996) found that the photo based approach improved sampling precision by $>35 \%$ over ground sampling for crown area and vegetation cover estimates. A combined large and small scale air-photo sampling approach for vegetation cover mapping was explored by Pitt et al. (2000) in leaf-on conditions. Large scale 1:500 photos were used to train an interpreter on the properties of vegetation classes in the smaller scale 1:5000 photos, which covered the entire sample area. A set of 1:500 photos used for validation showed that tall, mid, and low shrub, grass, and herbaceous species cover were estimated to within $5-10 \%, 70 \%$ of the time.

Operational practice of these methods has not been widely undertaken because photo acquisition and visual analysis are either highly specialized, time consuming, or subjective, demanding specially trained personnel and equipment (King, 2000). Thus, more easily applied automated or semi-automated image interpretation methods that removelminimize subjectivity and maximize accuracy and precision are required. Further, new sensor developments provide improvements for all stages of image acquisition, processing and analysis. With digital, instead of film-based cameras, image exposure can be controlled during acquisition and image quality can be assessed in flight ensuring that a high quality product is obtained. There are no costs of film and film development or scanning with digital sensors. The response of digital sensors to incoming radiation is linear, while film is log-linear, making the former more applicable to quantitative image analysis (King, 1995). 


\subsubsection{Medium Resolution Approaches to Regeneration Assessment}

With moderate resolution imagery $(5 \mathrm{~m}-100 \mathrm{~m}$ pixels $)$, several studies have found a relation between vegetation abundancelage class and the spectral response of regenerating forests. Horler and Ahern (1986) found SWIR bands 5 and 7 of Landsat TM ( $30 \mathrm{~m}$ resolution) to be sensitive to forest vegetation density, especially in the early stages of a regenerating clear-cut. Coleman et al. (1990) found that Landsat TM bands 5 SWIR, 4 - NIR, and 3 - red could differentiate age classes (6-10,11-20, 21+ years) of young pine plantations, but not species. Fiorella and Ripple, (1993) found a strong correlation between stand age and a structural index (TM bands 4/5) for Douglas fir forests $(r=0.98)$ and concluded that poor and well regenerated stands could be differentiated after age 15. Jakubauskas (1997) showed that textural properties of Landsat TM could be used to separate lodgepole pine age classes of post disturbance regeneration, dense small diameter trees, and mature classes. Shaw et al. (1998) found a strong relation between regenerating Scots pine (Pinus sylvestris) cover and spectrometer measurements $\left(r^{2}=0.84\right)$. Wulder et. al. (2004) developed a multiple regression model for boreal forest age prediction using Landsat ETM + bands and the Tassel Cap transformation $\left(\mathrm{R}^{2}=0.68\right.$, root mean square error $=2.4$ years).

Results with moderate resolution imagery have shown the potential for monitoring basic regeneration properties. This may provide the means for very large area monitoring and flagging of areas with low regeneration cover. However for forest management treatment decisions, additional information regarding the spatial distribution of crop species is needed as well as information on non-crop competitors. 


\subsubsection{High Resolution Approaches to Regeneration Assessment}

High resolution imagery has been shown to provide the necessary detailed information required for regeneration assessment (Section 3.4.4). However, the major limitation has been extracting the information from imagery in a reliable and timely manner. Digital image processing of high-resolution imagery may provide a solution to this problem if suitable algorithms can be developed. To date, few studies have made use of quantitative digital image analysis for extraction of information regarding regeneration properties. Brown and Fletcher (1999) used classification and an optimal spacing algorithm described in Price and Davidson (1999) to evaluate $60 \mathrm{~cm}$ Compact Airborne Spectrographic Imager (CASI) data for regeneration assessment and found that wellspaced estimates of trees greater than $75-80 \mathrm{~cm}$ tall could be made. However, the results underestimated field measurements by $\sim 30 \%$. They also concluded that conifer species differentiation was not reliable at this resolution. Haddow et al. (2000) found a systematic decrease in the ability to classify conifers with increasing competition levels in leaf-on imagery. They also evaluated the relation between spectral response and competition leaf-area index and percent cover. Results showed that the red band spectral response was the most significantly related to these measures. Gougeon and Leckie (1999) applied an automated tree detection and delineation algorithm originally developed for mature forests to forest regeneration of 3-10 years of age for crop tree stem detection and crown diameter measurement. They found that stand average stem density measurement errors ranged from $11-43 \%$, while crown diameter measurement errors ranged from 7-9 \%. This is the only previous study that applied such detection-delineation techniques to 
regenerating forests. Previous research using automated tree detection and delineation techniques in mature forests is described in Sections 5.1-5.4.

\section{OVERVIEW OF RESEARCH TOWARDS THE DEVELOPMENT OF A REMOTE SENSING BASED REGENERATION MONITORING SYSTEM}

\subsection{INTRODUCTION}

The main purpose of this research was to develop a remote sensing based approach for regeneration assessment to improve spatial sampling capabilities and provide information with greater precision and timeliness for use by managers. There are numerous means by which a remote sensing based regeneration assessment procedure could be devised. Previous research in moderate resolution and visual analysis of aerial photography has shown that useful information on crop trees can be extracted from either image format. However, for regeneration assessment of young trees (e.g. 2-15 years old) high-resolution imagery is required to estimate tree size and spatial distribution properties. The analysis also requires robust algorithms that can process large amounts of data rapidly, which has been the main difficulty with visual analysis approaches. To address these problems, two primary research objectives were identified:

1. Develop and evaluate an automated method for conifer crop tree information extraction;

2. Develop and evaluate an automated method for woody deciduous competition information extraction. 
Both objectives were constrained to using high resolution $(<15 \mathrm{~cm})$ airborne sensor imagery. Leaf-off (i.e. when deciduous and herbaceous vegetation is not green) imagery is best for conifer detection and delineation, but it is not optimal for competition assessment because only branches and stems of deciduous vegetation are captured in the imagery, rendering it difficult to identify and quantify. In leaf-on imagery, leaves of competition make identification of competition presence much easier, but these leaves also hide crop trees from the sensor view. Further, in leaf-on conditions, conifer crowns can be difficult to decipher from the surrounding green herbaceous vegetation (Haddow et al., 2000). For combined crop and competition assessment, both leaf-off and leaf-on imagery could be used, but the high cost of dual acquisition and differential timing hinder the potential for operational acceptance of any proposed methods. Therefore, in this research, methods for crop and competition assessment were developed using only leafoff imagery.

Three different study areas located in Northern Ontario were utilized representing a range of regeneration conditions. They consisted of an experimental study site with highly controlled tree size and spacing, three young regenerating cutovers $(<10$ years of age $)$ with varying levels of competing vegetation, and three older cutovers ( $>14$ years of age), again with varying levels of competing vegetation.

\subsection{CROP TREE INFORMATION EXTRACTION}

For crop tree information, an automated tree detection and delineation approach was developed and evaluated (sections 5.1-5.4). As discussed in sections 5.1-5.4, the 
approach provides the means to extract several key types of information from highresolution imagery for regeneration assessments. Tree detection can provide estimates of crop-tree abundance and spatial pattern useful for evaluating density and stocking objectives, as well as inputs for spatial based growth modeling. Crown delineations can be used to enhance species composition inventory by improving individual species identification through analysis of within-crown spectral data (Gougeon, 1995b, Leckie et. al., 2003a; Leckie et al., 2005), spatial data (Haddow et al., 2000; Erikson, 2004), lidar data (Holmgren and Persson, 2003), and crown shape (Brandtberg, 1999). Research has also shown potential for disease damage mapping (Leckie et al., 2004). Further, delineated crown dimensions can be used to model tree structural variables such as height, volume, or biomass. (Culvenor, 2000; Hayward and Slaymaker, 2001; Persson et al., 2002; Pouliot et al., 2005).

In this research, the automated tree detection and delineation algorithm was designed to be optimal in the unique conditions of regenerating forests, which include:

1. Small trees;

2. High spatial variability of tree density;

3. High variability of tree size characteristics;

4. High variability of inter-tree spacing relative to tree size, resulting in trees growing in clumps or as single isolated objects;

5. Reduced vertical structure compared to that of mature forests. 
Considering these properties, the optimal approach requires very high-resolution imagery in order to detect small trees. In mature forests, algorithms have typically been applied in moderate to closed canopy conditions. In these conditions, crown radiometric morphology is largely controlled by shadowing effects between crowns forming local minima that have been used for crown delineation (Gougeon, 1995a; Gougeon and Leckie, 1999; Culvenor, 2000). However, in regeneration conditions, where crowns are not always bounded by shade, local minima were found to overestimate the crown boundary and local edges were more appropriate (Pouliot et al., 2002). Thus, although a few algorithms have been developed for mature forests, they could not simply be adapted for regenerating conditions and a new algorithm was required. Further, the development of the algorithm allowed for control of all stages of processing, providing the capacity to integrate new methods and other modifications as needed.

Four research papers were produced in the development and evaluation of a suitable algorithm that could be applied in regenerating forests. The first paper (Section 5.1) was designed to evaluate an initial detection and delineation algorithm in "idealized" regeneration conditions to determine an upper accuracy limit for automated and visually interpreted results, the radiometric properties of the crowns that could be used to improve detection and delineation, and to identify the major error sources. These "idealized conditions" were present in an existing competition study installed by the Ontario Ministry of Natural Resources near Sault Ste Marie, Ontario. The site included reduced inter-tree spacing, tree size, and background variability (no slash or ground vegetation and controlled competition species) compared to that found in more operational 
conditions. The results of this research and other published studies revealed that one of the main sources of error in automated tree detection is image scale. Thus, the second paper (Section 5.2) evaluated several approaches to reduce this scale dependence by determining an optimal image scale for detecting tree crowns. No method was found to be optimal in all conditions, but the best method from the range of conditions tested was selected for further development of the final algorithm. This method, referred to as the local maxima smoothing relation (LMSR), evaluates the relation between simulated scale and the number of crowns detected to determine the optimal global scale to be used in the analysis. Local scale optimization methods can provide better results, as scale is optimized for each crown object to be detected, but they tend to a require a higher degree of parameterization and were more sensitive to parameter values. The third paper (Section 5.3) extended the methodology to operational conditions likely to exist after clear-cut forest harvesting and replanting. Initial attempts of applying the algorithm developed in Section 5.1 under idealized conditions showed very poor performance in these more operational conditions. Thus, a more robust algorithm was developed by combining the initial algorithm with approaches published at the same time for mature forests. This algorithm was rigorously evaluated in regards to the different error sources, including tree size, crown overlap and competition presence. The fourth paper (Section 5.4) is a large-area operational application of the latest algorithm. It represents a true operational pilot study, using scanned aerial photography and fixed wing aircraft for the data acquisition. 


\subsection{NON-CROP COMPETITION INFORMATION EXTRACTION}

Information regarding crop trees is, on its own, useful information, as it pertains to the trees desired for the future regenerated forest. However, information regarding non-crop vegetation is also important to determine if non-crop trees are significantly impeding crop growth (requiring silvicultural intervention) and for other ecologically based evaluations such as wildlife food and shelter. The objective of this research was to develop a method complementary to the automated tree detection and delineation approach used for crop tree assessment to extract information on competition from leaf-off imagery. In this research, visual and automated extraction techniques were evaluated in a single research paper (Section 5.5). Visual methods consisted of digitizing hardwood stems and outlining clumps of shrubs. Automated extraction made use of spectral ltextural classification to identify hardwood and shrub pixels. Line detection was used to further refine hardwood stem extraction and provide capability for determination of stem length. The measures of hardwood and shrub abundance extracted from the imagery were then modeled against field measured competition abundance. Results revealed that strong relations existed for visual and automated extraction, but visual extraction was superior. Thus, it was recommended that the automated extraction be used as an initial coarse filter, and further refinement of areas close to a competition threshold be reassessed using the visual approach. This research is the first to address this topic and it is recognized that there is room for considerable refinement to the current methodology. 


\title{
5. RESEARCH PAPERS
}

\subsection{DEVELOPMENT AND EVALUATION OF AN AUTOMATED TREE DETECTION AND DELINEATION ALGORITHM IN OPTIMAL REGENERATION CONDITIONS REGARDING REGENERATION STRUCTURE}

Citation: Pouliot, D.A., D.J. King, F.W. Bell, and D.G. Pitt (2002). Automated tree crown detection and delineation in high-resolution digital camera imagery of coniferous forest regeneration. Remote Sensing of Environment, 82: 322-334.

\begin{abstract}
Ensuring successful forest regeneration requires an effective monitoring program to collect information regarding the status of young crop trees and nearby competing vegetation. Current field based assessment methodology provides the needed information, but is costly, and therefore assessment frequency is low. This often allows undesirable forest structures to develop that do not coincide with management objectives. Remote sensing techniques provide a potentially low cost alternative to field based assessment, but require the development of methods to easily and accurately extract the required information. Automated tree detection and delineation algorithms may be an effective means to accomplish this task. In this study, a tree detection-delineation algorithm designed specifically for high-resolution digital imagery of six year-old trees is presented and rigorously evaluated. The algorithm is based on the analysis of local
\end{abstract}


transects extending outward from a potential tree apex. The crown boundary is estimated using the point of maximum rate of change in the transect data and a rule base is applied to ensure that the point is contextually suitable. This transect approach is implemented in both the tree detection and crown delineation phases. The tree detection algorithm refines the results of an initial local maximum filter by providing an outline for each detected tree and retaining only one local maximum value within this outline. The crown delineation algorithm is similar to the detection algorithm, but applies a different rule set in creating a more detailed crown outline. Results show that the algorithm's tree detection accuracy was better than that using commonly applied fixed window local maximum filters; it achieved a best result of $91 \%$. For the crown delineation algorithm, measured diameters from delineated crowns were within $17.9 \%$ of field measurements of diameter at the crown base on an individual tree basis and within $3 \%$ when averaged for the study. Tests of image pixel spacings from $5 \mathrm{~cm}$ to $30 \mathrm{~cm}$ showed that tree detection accuracy was stable except at the lowest $(30 \mathrm{~cm})$ resolution where errors were unacceptable. Delineated crown diameter accuracy was more sensitive to image resolution, decreasing consistently and non-linearly with increasing pixel spacing. These results highlight the need for very high resolution imagery in automated object-based analysis of forest regeneration.

\subsubsection{Introduction}

Successful regeneration of coniferous species is critical to forest sustainability in boreal regions. Conifers are the dominant species in this biome, but can be difficult to reestablish after disturbance due to slow growth and sensitivity to competing vegetation. 
Timely information regarding their stocking levels, health, and competing species abundance is required for effective treatment decisions. Current field survey methods are labour intensive and costly, resulting in low sample coverage and frequency. As a consequence, undesirable forest structures can develop between sampling periods that are difficult to restore to desired conditions. Remote sensing has potential to provide, at lower cost, forest information with greater coverage than is attainable using field sampling. Early work in the development of remote sensing methods for regeneration assessment focused on manual analysis of large-scale photography (LSP). Results have shown that reliable estimates of stocking, species, crown area, health condition, and stratification of key vegetation complexes can be made (Goba et al., 1982; Hall, 1984; Hall and Aldred, 1992; Pitt and Glover, 1993; Pitt et al., 2000). However, operational practice of these methods has not been widely undertaken because LSP acquisition and analysis are either highly specialized, time consuming, or subjective, requiring specially trained personnel and equipment (King, 2000). Thus, methods to automate and simplify acquisition and analysis are required for an effective remote sensing based assessment methodology.

Potential improvement of the utility of remote sensing for regeneration assessment may be obtained through the application of automated tree detection-delineation algorithms. Estimates of tree spatial pattern, tree abundance, crown size, and canopy structure can be made given accurate detection and delineation of crowns. Isolation of individual trees also provides for improved species classification through analysis of within-crown spectral data (Gougeon, 1999), spatial data (Haddow et al., 2000), and crown shape 
(Brandtberg, 1999). Further, crown dimensions can be used to model tree structural variables (e.g., stem diameter, height, and biomass) useful in forest inventory and evaluation of growth success (Culvenor et al., 1999; Hayward et al., 2001). Automated tree detection and crown delineation algorithms developed to date have been applied almost entirely in mature forest conditions. A summary of these is given in the next section with details of the forest conditions in which they were developed and tested. Some algorithms work well in relatively closed canopies, while others are more suited to individual trees with little crown overlap and full rounded crowns. Most have been developed for imagery of $0.5 \mathrm{~m}-2 \mathrm{~m}$ pixel spacing, which in mature forests, results in crown diameter:pixel spacing ratios of less than about 8:1. In remote sensing of young regenerating trees, however, Pitt et al. (1997) concluded that only the very highresolution capabilities of aerial photography and digital cameras would be suitable. In this paper, a detection-delineation algorithm is presented and evaluated using airborne imagery of 5-15 cm pixel spacing and 6-10 year old conifer trees planted at $1 \mathrm{~m}$ spacing. In these conditions, the crown diameter:pixel spacing ratio is about 17:1 and individual tree crowns are well resolved with a large number of pixels and high data variance within each crown. The algorithm was designed specifically to improve upon current tree detection algorithms by identifying and removing false positives corresponding to resolved branch clusters within a crown, and to accurately detect crown boundaries while avoiding within-crown shadow edges. 


\subsubsection{Objectives}

The objectives of this research were to:

1. Develop a tree detection and delineation algorithm for very high resolution remote sensing of regenerating coniferous forests.

2. Conduct a detailed evaluation of the accuracy of the algorithm against field data of known tree locations and crown diameters.

\subsubsection{Previous Research in Tree Detection and Delineation}

\subsubsection{Tree Detection and Delineation Algorithms}

At present, detection and delineation algorithms are based on two distinct spectral properties of tree crowns: the association of a tree apex with a local maximum image brightness value, and delineation of the crown boundary by local minima brightness values. Visually, this spectral crown structure is analogous to that of an upward pointing cone or mountainous shape when viewed in three-dimensions. Approaches to tree detection have attempted to identify local maxima using: 1. enhancement and thresholding (Gougeon and Moore, 1988; Dralle and Rudemo, 1997; Walsworth and King, 1998) where a global image operation such as smoothing or high pass filtering is applied and the resulting pixel brightness values within a defined range are extracted as estimates of tree locations, 2. template matching (Pollock, 1998) involving the correlation between a geometric-radiometric tree crown model to image data, 3 . multiscale analysis (Brandtberg and Walter, 1998) where the occurrence of edges over several image scales is examined to define a region in which the brightest pixel value is taken as the tree apex, 
and 4. local maximum filtering (Culvenor, 2000; Gougeon, 1997; Niemann et al., 1999; Wulder et al., 2000) where the maximum pixel brightness value in a moving window sample of a specified size is taken to represent the tree apex. Tree delineation has been accomplished by: 1 . outlining a network of minimum image values, known as valley following (Gougeon, 1995a); 2. region grouping (Brandtberg and Walter, 1998; Walsworth and King, 1998), involving the identification of groups of similar neighbouring pixels; and 3. a combination of these two (Culvenor, 2000).

Specifically in this research, variable window local maximum filtering methods identified by Wulder et al. (2000) were expanded to improve detection and provide for automated crown delineation. With local maximum filtering, a moving window is passed over the image and the location of the maximum value in each window is recorded. These windows can be overlapping or not, the non-overlapping requirement ensures that pixels in the image are evaluated within only one sample window frame, reducing detection error caused by portions of bright trees being repeatedly detected with overlapping windows. However, with either moving window strategy, accurate results are dependent on the identification of an appropriate sample window size. Large windows result in missed trees (omission error) because the window contains multiple tree apexes. With small window sizes, too many apexes are identified (commission error) because the small window does not always contain a true tree apex. Wulder et al. (2000) addressed this problem by creating variable window sizes for local maximum evaluation. The size of the window was based on either the range from a semivariogram or the local slope break (i.e. inflection point) for the average of eight radiometric transects extending outward from 
the pixel being processed. The approach taken in this research is conceptually similar, but is mechanically different in: 1 . the analysis of transect data (maximum rate of change rather than inflection point), 2. creating a circular rather than square sample window, and 3. the use of a greater number of transects (up to 360 if desired).

\subsubsection{Tree Detection and Delineation Accuracy Assessment}

Evaluation of these algorithms is typically based on individual tree or aggregated assessments. Individual assessments compare automated and reference data for single trees, whereas aggregation averages automated and reference data within a given area for comparison (e.g., the number of trees detected per hectare). Accurate individual evaluations are preferred as more detailed information is provided for decision making $\backslash$ analysis, however, this is difficult to achieve. Aggregated estimates generally result in higher accuracies than individual estimates as errors of omission and commission in tree detection, or overshoots and undershoots in crown boundary delineation tend to be averaged out in the aggregation process.

Tree detection accuracy has been well researched and is commonly performed at an individual tree level using reference data consisting of tree locations visually interpreted from the imagery (Gougeon, 1995a; Brantberg and Walter, 1998; Niemann et. al., 1999; Pollock, 1999; Walsworth and King, 1998). For example, Gougeon (1995a) found that the total number of correctly identified trees in a mature forest plantation ranged between $35 \%$ for red pine and $85 \%$ for a mixed stand of red pine and white spruce. Comparisons with field data (Brandtberg and Walter, 1998; Wulder et al., 2000) are less common due 
to the difficulty and associated costs of identifying tree locations in the field. One example is presented in Wulder et al. (2000) who, working in natural and plantation forests, reported a best result of $62 \%$ correctly identified trees with $11 \%$ commission error using a variable window detection algorithm. Comparing automated and field estimates at an aggregated level is more feasible (Gougeon, 1995a; Gougeon and Leckie, 1999), as only the number of trees in the plot is required, not their exact location. However, the number of missed and falsely identified trees cannot be evaluated for algorithm testing and refinement. This type of evaluation was used by Gougeon and Leckie (1999) to determine the accuracy of a valley following algorithm applied to several species and age classes of forest regeneration. Results showed that average error for the different conditions ranged from $43 \%$ to $13 \%$ for 7 and 8 year-old jack pine stands respectively.

Tree delineation accuracy has not commonly been evaluated because of the difficulty of precisely measuring tree crowns in the field. Field based crown measurements are subject to errors relating to how well field personnel can project the crown boundary down to a measuring device and identification of a suitable boundary point to measure for tightly overlapping or irregular crowns. Instead, studies in natural forests have commonly used crown diameter to evaluate delineation accuracy. Pollock (1999) evaluated crown diameter accuracy for individual trees, but results were only compiled for dominant tree crowns. More importantly, the template matching algorithm that was evaluated does not actually delineate the crown, which is required to isolate spectral data for enhanced species classification. Brandtberg and Walter (1998) evaluated crown diameter accuracy 
for plot-level data and found no relation between the automated and ground estimates. Culvenor (2000) validated a delineation algorithm using crown area and simulated forest imagery where defined crowns of known area served as the reference data. In conifer forest regeneration conditions, small tree sizes provide an ideal means to evaluate delineation accuracy as individual trees can be more easily identified in the field and crown diameter measurements can be made quickly and with greater precision than in mature forests. However, only one study to-date has conducted such validation. Gougeon and Leckie (1999), using a hybrid delineation algorithm based on Gougeon's original valley following approach, found an aggregated study-wide error in average crown diameter of $8.5 \%$, but it was also determined that the $30 \mathrm{~cm}$ pixel spacing was too large to detect and delineate the smaller trees present.

A critical factor found to affect all tree detection and delineation algorithms is withincrown brightness variation. In high-resolution imagery, within-crown variation is large due to the effect of branches and branch shadow patterns on the spectral response of the crown. This causes crowns to deviate from the conic shaped model, making detection and delineation more complex. Decreasing the image resolution averages spectral data over a larger areal unit and reduces this effect. However, at lower image resolutions crown boundaries become less distinct, making them harder to identify (Figure 5.1.1). 


\begin{tabular}{|c|c|c|}
\hline & $5 \mathrm{~cm}$ pixel spacing & $15 \mathrm{~cm}$ pixel spacing \\
\hline $\begin{array}{l}\text { Crown } \\
\text { diameter } \\
95.5 \mathrm{~cm}\end{array}$ & & \\
\hline $\begin{array}{l}\text { Crown } \\
\text { diameter } \\
43.0 \mathrm{~cm}\end{array}$ & & \\
\hline
\end{tabular}

Figure 5.1.1: Three dimensional view of large and small tree crowns in the near infrared band for pixel sizes of $5 \mathrm{~cm}$ and $15 \mathrm{~cm}$. This figure illustrates the greater within crown variability of large crowns with the $5 \mathrm{~cm}$ pixel size and the less distinct crown boundary of smaller crowns at the $15 \mathrm{~cm}$ pixel size.

To characterize the generalization of crown shape at different image resolutions we propose the use of a one-dimensional ratio of the average crown diameter to the pixel size or the range of crown diameter to pixel size ratios for the range of crown sizes in a study. For example, in Figure 5.1.1 this ratio is approximately 19:1 and 6:1 for the large crown and 8:1 and 3:1 for the smaller crown at the high and low image resolution, respectively. It is evident from this example that ratios below 3:1 do not retain distinct crown boundaries and ratios above or near 19:1 may contain too much within crown variation for accurate delineation. Thus, this ratio can be used as a guide to determine the most appropriate image resolution to use for detectionldelineation based on an estimate of the average crown size or expected range of crown sizes (e.g., $95 \%$ bounds) in the study area. To date, with the exception of Brandtberg and Walter (1998), average crown diameter to pixel size ratios have typically been small (e.g., about 10:1 in Gougeon, 1995a; 5:1 in Culvenor et al., 1999; 4:1 in Gougeon and Leckie, 1999). The optimum 
ratio for a given algorithm will also be dependent on its sensitivity to within crown variation and boundary brightness gradient. However, reporting the ratio in tests is very useful, as it allows for comparison of algorithm performance over a range of ratios and for comparison of studies of similar ratios conducted in different forest conditions.

\subsubsection{Methods}

\subsubsection{Study Area}

The study area consists of an experimental arboretum located outside of Sault Ste. Marie, Ontario $\left(46^{\circ} 33^{\prime} \mathrm{N}, 84^{\circ} 27^{\prime} \mathrm{W}\right)$, established in 1994 as part of an on-going effort to identify the effects of various levels of vegetative competition on black spruce (Picea mariana) and jack pine (Pinus banksiana) crop trees. The site is arranged into three blocks based on soil properties: sand, clay and loam. Each block contains ten $42 \times 14 \mathrm{~m}$ subplots of key competition species established at several densities. Crop species (spruce and pine) were established at a constant spacing of $1 \mathrm{~m}$ in all subplots. In this study, to minimize the effect of known error sources on the detection and delineation algorithm, the selection of suitable subplots was constrained by three factors. First, subplots with competing deciduous species were removed to prevent detectionldelineation error occurring from the effect of overtopping competition masking the spectral characteristics of the crop trees. Second, only image data within seven degrees of nadir were extracted to avoid variations in shadow-crown geometry across the image caused by the non-vertical position of the sun and variable optical view angle. The seven degree requirement was selected based on an in depth analysis of radiation geometry conducted for mature forests by Culvenor 
(2000). Third, only the subplots containing spruce crop trees were used, as intense intra specific competition in jack pine subplots rendered individual crowns unidentifiable in the imagery. In total, eight subplots (198 trees) were extracted from the loam, sand, and clay blocks and used to create the image subset for analysis. In previous research at the site, Haddow et al. (2000), using $2.5 \mathrm{~cm}$ pixel colour infrared digital camera imagery, were able to automatically classify and count these conifers at age 2 years (1996) in the low to no competition plots (similar to a leaf-off condition) with over $90 \%$ accuracy, and to model cover and leaf area index of competing vegetation with standard errors of 10-20 $\%$.

\subsubsection{Ground Data}

For validation of crown delineation, crown diameters were measured in late summer 1999 by Ontario Ministry of Natural Resources staff. Measurements were made in N-S and E-W directions at the crown base of the inner 25 trees in each subplot and the arithmetic average taken to provide a single summary value for comparison with image delineations. Crown diameters ranged from $16 \mathrm{~cm}$ to $145 \mathrm{~cm}$ with a mean of $85 \mathrm{~cm}$ (Figure 5.1.2). For validation of detection results, tree positional data were not required, as the initial planting arrangement of $1 \mathrm{~m}$ spacing was known and was identifiable in the imagery. 


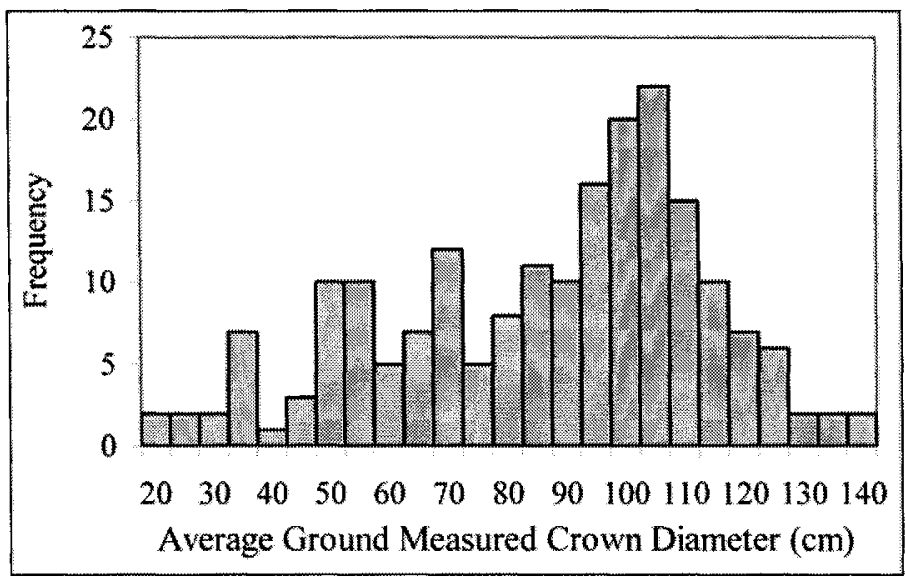

Figure 5.1.2: Histogram showing the distribution of field measured crown diameters (average of N-S and $\mathrm{E}-\mathrm{W}$ directions for each tree) used in the analysis.

\subsubsection{Image Data}

Images of the study site were acquired in a leaf-off condition on April 25, 2000 between 12:00 - 14:30 using a Kodak DCS 460 CIR digital camera. Over this period the sun zenith angle ranged from 52 to 56 degrees. Three spectral bands were acquired in the green $(500-600 \mathrm{~nm})$, red $(570-780 \mathrm{~nm})$, and near infrared (NIR, 710-800 nm). To achieve the desired $5 \mathrm{~cm}$ pixel spacing, a flying height of $196.6 \mathrm{~m}$ and lens focal length of $35 \mathrm{~mm}$ were used. The shutter speed was set at $1 / 4000 \mathrm{~s}$ to optimize spatial detail and exposure, and to minimize image motion (approx. $1 / 4$ pixel in each exposure).

\subsubsection{Tree Detection and Delineation Algorithm}

\subsection{Detection}

The algorithm developed in this study consists of two separate programs, one for detection and one for delineation. Together these programs are referred to as 'Local 
Maximum Refinement and Delineation Algorithm' or LMRDA. The tree detection phase consists of the seven steps denoted A to $\mathrm{G}$ in the following description and in Figure 5.1.3. In the first stage (A), image pre-processing is undertaken to enhance the crown apex (or local maximum) distinctiveness, minimize the amount of bright crown non-apex pixels, and suppress the spectral response of bright soil in the imagery for tree detection. Amongst several processing types tested, including common vegetation indices, the best results were obtained using an absolute difference image of the NIR and red spectral bands. A moderate amount of Gaussian smoothing (filter size $=11 \times 11, \sigma=5$ ) is also applied to simplify crown form and reduce image noise (Figure 5.1.4).

In stage (B) a non-overlapping local maximum filter is applied to the image. The window size is specified by the user, but should be small compared to the average crown size in the image, as the algorithm only works to reduce the occurrence of commission errors. In this study, window sizes of $15 \times 15$ to $30 \times 30$ pixels were evaluated. 


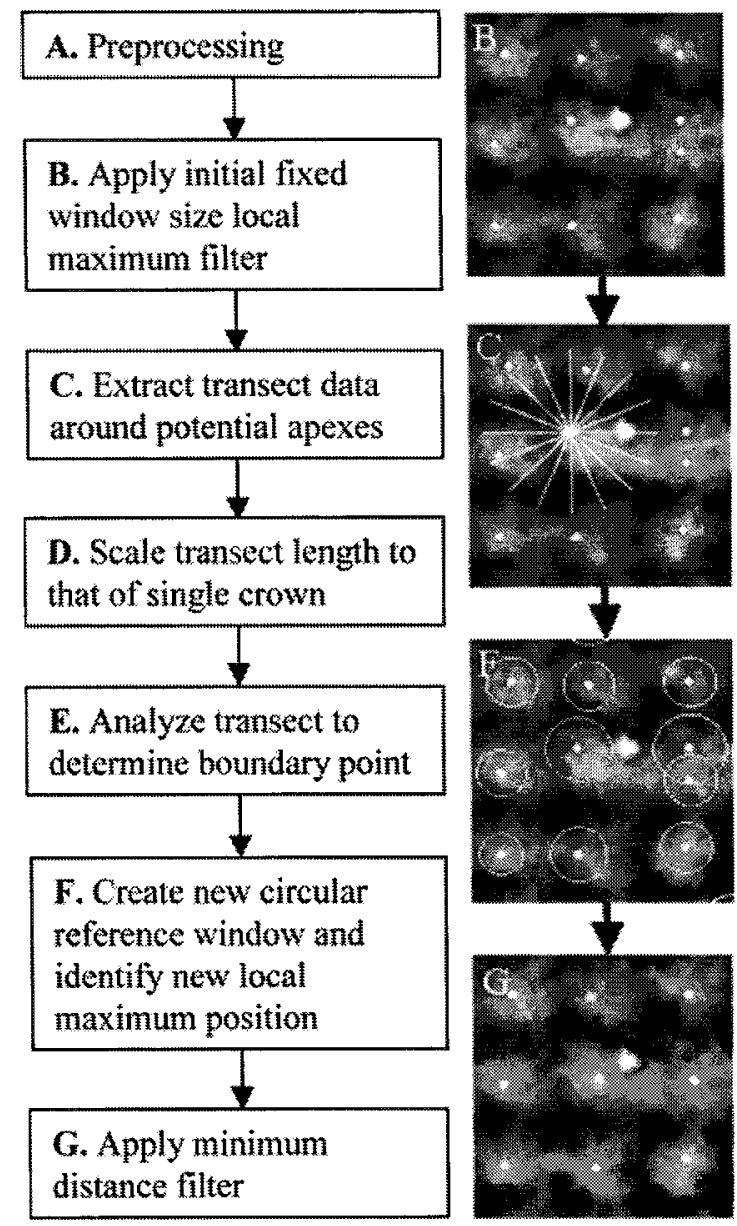

Figure 5.1.3: Overview of tree detection algorithm process. Stages of the algorithm are lettered A-G. On the left is the complete process, on the right are images representing output at critical stages (note Stage letter in upper left of each image).

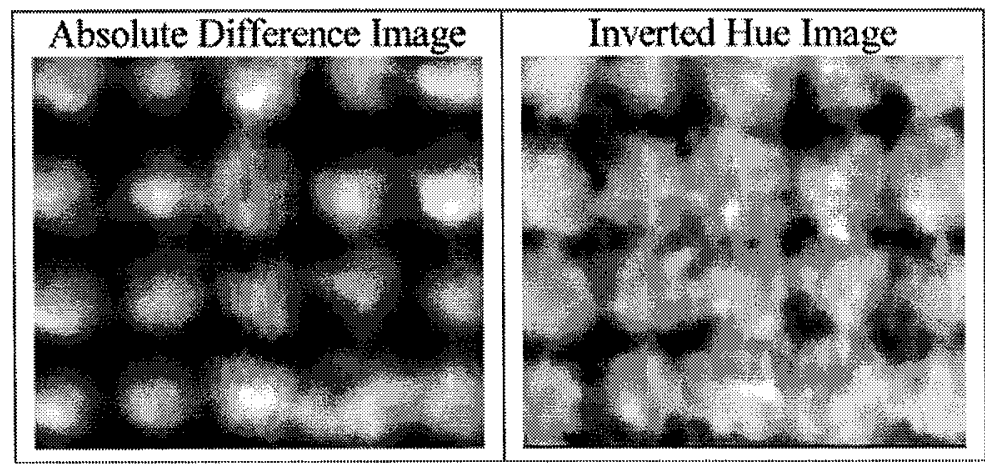

Figure 5.1.4: Example images showing image pre-processing for optimal detection and delineation: absolute difference image used in crown detection (left), and inverted hue image used in crown delineation (right). 
In the third stage $(C)$ image data surrounding a potential tree apex are extracted using the transect sampling scheme depicted in Figure 5.1.3C. The number of transects used in the analysis is selected by the user and can be set as high as 360 and as low as 4 . Using more transects can improve results, however, the improvement saturates quickly as a reasonable crown radius for detection purposes can be estimated with approximately 15 20 transects. Further, the use of more transects requires significantly more processing time. The initial transect length used to extract image data is defined by the user, but should be set so that it is larger than the largest expected crown radius in the image.

Transect scaling, determining the best transect length for tree edge detection, is accomplished in stage (D). The transect data are modeled with a fourth order polynomial and data from the end of the transect are iteratively removed one pixel value at a time until a user defined $r^{2}$ value is reached for the original and modeled data. This process provides a good generalization of the image data while appropriately scaling the transect to be slightly larger than the actual crown radius. Figure 5.1.5 shows how the initially extracted transect data are reduced to a suitable length for crown edge detection using the $r^{2}$ value from a fourth order polynomial fit. The choice of the $r^{2}$ cut-off depends on the variability of the data and pixel spacing. At large pixel spacings, a small number of transect pixel values are extracted. Thus, using high $r^{2}$ values $(0.95)$ may leave too few remaining values for crown edge detection if some are removed in this process. Similarly, in the case of noisy data or data with high variability, using a high $\mathrm{r}^{2}$ value can result in a large amount of transect data being removed in order to achieve the desired polynomial fit. Smoothing with a low-pass filter as in stage (A) can be an effective means to reduce 
image noise allowing for higher $\mathrm{r}^{2}$ cut-off values to be used. In this study, user-specified $\mathrm{r}^{2}$ thresholds ranged from 0.90-0.98 depending on the image pixel spacing.
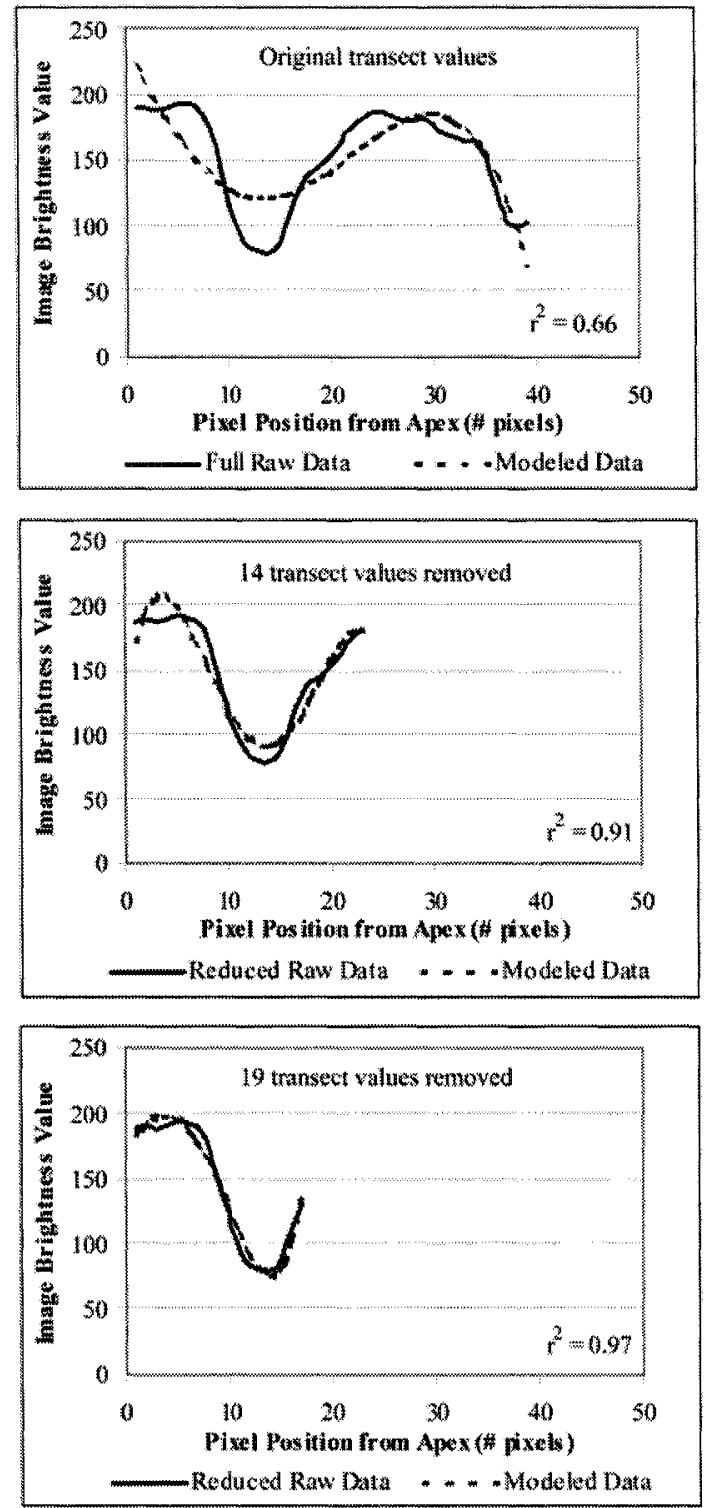

Figure 5.1.5: Example of transect scaling process, (Stage D of the algorithm). The top figure shows the full transect length while the bottom figure shows the reduced transect length determined using a best fourth order polynomial model fit criterion $\left(r^{2}=0.95\right)$.

In the fifth stage $(\mathrm{E})$, the crown radius for each transect is estimated by examining the modeled data of the reduced transect to identify the maximum rate of change in the 
transect image values. The maximum rate of change is an edge detection operator that is calculated by sequentially subtracting transect brightness values from the preceding value and taking the maximum of these values as the detected edge (i.e., maximum value in the first derivative for a defined set). The use of the maximum rate of change position requires appropriately scaled transects (from Stage D) so that the most significant boundary (highest rate of change) identified is indeed that for the crown under consideration and not for a more abrupt edge of a neighbouring tree. Inflection points (the change of sign of the second derivative) were also tested for this stage of the algorithm but were rejected because there were often more than one identified for a given transect, representing small within-crown edges.

Circular reference windows are created in stage $(\mathrm{F})$ by estimating the crown radius based on the average distance from the apex to the crown boundary identified in stage (E). However, before averaging, extreme values are removed by converting distance estimates to $\mathrm{z}$-score values and removing values greater than 2 (representing data that do not fall within $95 \%$ of the mean). $Z$-scores were calculated as:

$Z=\left(x_{i}-x\right) / s$

where $x_{i}$ is the distance to the most significant edge of transect $i, x$ is the mean distance and $s$ is the standard deviation for the distances. The remaining distances are averaged to define a radius for the new circular reference window. The spectral data within each of 
the newly defined circular windows are then examined to determine the local maximum value, which is taken as the final tree location.

In the final stage $(G)$, a minimum distance filter is applied that returns the centroid of a group of pixels identified as local maxima in the previous steps that occur within a user defined distance of a point, a procedure similar to that developed by Culvenor (2000). The purpose of the filter is to remove apexes that occur closer together than the physical structure of the trees in the image would allow.

\subsection{Delineation}

Image pre-processing for delineation was different from that applied for detection. To enhance the image spectral properties for tree delineation an inverted hue image (Figure 5.1.4) was used, as it provided a useful spatial gradient for crown edge detection and maintained the distinct crown structure on the shaded side of the crown. The absolute difference image used for detection was not useful in delineation as it masked the spectral response on the shaded side of the crown causing crown boundaries to be underestimated. Gaussian smoothing was also applied to reduce image noise (filter size $=11 \times 11, \sigma=5$ ).

The delineation component of the LMRDA is based on the same design as the detection program, but returns the position of the crown edge for each transect rather than the distance to the edge. If the distance for a detected edge is smaller than a user specified

minimum edge distance the point is removed. The remaining edge points are then used to 
construct a polygon representing the crown boundary. In this study, the minimum edge distance used was 1 pixel.

In some cases, the delineated polygon can be improved by removing unlikely internal and external angles. In the case of spruce trees, the round crown shape makes acute polygon angles unlikely and, therefore, they should be removed. Figure 5.1.6 shows the effect of sequentially removing larger polygon angles. Without angle removal, the delineated polygon appears to be most similar to a natural crown shape, but it has some extended points and rapid indentations that do not match the crown boundary. Applying the $40^{\circ}$ polygon angle removal reduces the presence of these points providing a generalized representation of the crown shape. Determination of the most suitable angle removal is left to the user; in this study angles of less than $20^{\circ}$ were removed. However, if trees have large branches, it may be necessary to include polygon angles of less than $20^{\circ}$ in order to make rapid directional changes in following the crown boundary. The choice of the size of the angle to filter is in part related to the number of transects used in generating the polygon. With more transects, larger polygon angles can be removed, as several polygon vertices should survive the filtering process. When using a smaller number of transects, care must be taken not to remove too many points as the polygon shape may be dramatically altered if not removed completely. 


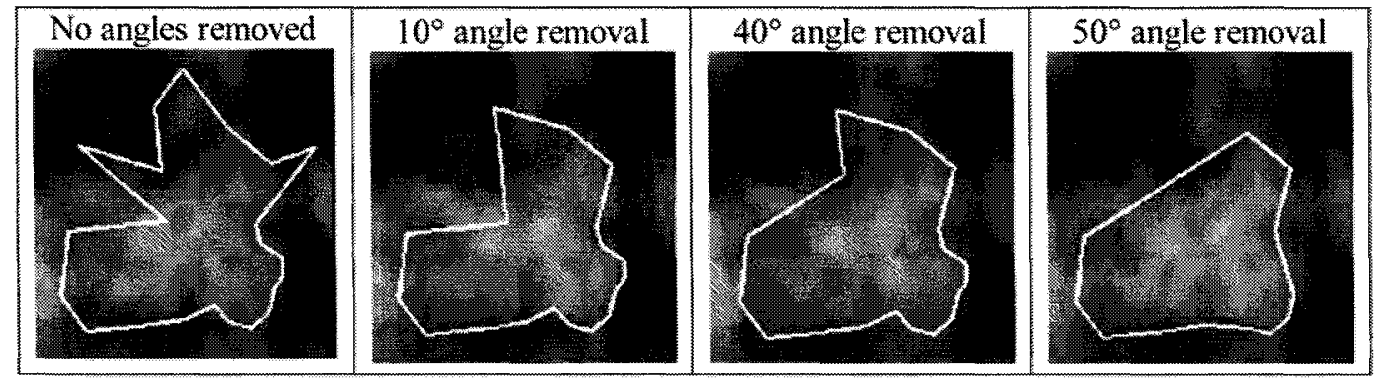

Figure 5.1.6: Effect of removing points that cause extreme internal polygon angles in delineated crowns.

\subsubsection{Detection and Delineation Algorithm Evaluation}

The tree detection and delineation algorithms were tested separately so that the error of each could be assessed independently on an individual tree basis and for aggregated data.

\subsection{Detection Accuracy}

Detection accuracy was assessed by comparing a truth map based on prior knowledge of tree locations (experimental design) and detected apexes. For each known tree, a single detected apex within the boundary of the crown was chosen to represent the crown and the remainder, if any, were counted as commission errors. Commission error was also counted when a local maximum point identified an image object other than a tree crown. Omission errors were counted when no apex was detected within the boundary of a known crown. The detection algorithm accuracy was compared to accuracies produced by ordinary local maximum filters (with no additional refinement) for fixed window sizes ranging from $3 \times 3$ to $30 \times 30$ pixels. Besides errors of omission and commission, overall accuracy of each detection method was defined as: 
$A I=[(n-o-c) / n] \times 100$

where $A I$ is an accuracy index in percent, $O$ and $C$ represent the number of omission and commission errors, and $n$ is the total number of trees in the image to be detected. The purpose of the index is to count all error against the correct number of trees to be detected. This provides a single summary value for comparison of detection results.

\subsection{Delineation Accuracy}

For each delineated tree crown, dimensions were extracted in the same two directions as the ground data using a program written to measure the delineated polygon diameter starting from the polygon centroid determined using the minimum bounding box method. These two values were averaged (arithmetic) and compared with the average ground diameter estimates. In addition, tree crowns were manually delineated by the first author from the screen display of the original $5 \mathrm{~cm}$ imagery and their resulting diameters were compared to the ground data. To evaluate the individual crown measurement error, the root mean square error (RMSE) of the crown diameters as a percentage of the mean true diameter was calculated as:

$R M S E \%=\frac{\sqrt{\frac{1}{n} \sum\left(P_{i}-O_{i}\right)^{2}}}{\bar{O}} \times 100$ 
where $O_{i}$ are the ground diameter measurements, $\bar{O}$ is the mean value of ground diameters, $P_{i}$ are delineated crown diameters, and $n$ is the number of observations. To evaluate aggregated error on a stand level the mean differences between the ground and delineated crown diameter measurements were calculated by subtracting the average of the two measurements (ground - automated; ground - manual) for the entire dataset and expressing these differences as a percentage of the mean of the ground data. Scatterplot analysis, of the absolute difference between image and ground measured crown diameters (y-axis) vs. ground measured crown diameter (x-axis), was also conducted to identify the systematic and random error components for a given delineation.

To determine the influence of image resolution on detection and delineation accuracy, the original untransformed imagery was resampled by averaging pixels within $2 \times 2,3 \times 3$, and $6 \times 6$ sample windows to produce image pixel spacings of $10 \mathrm{~cm}, 15 \mathrm{~cm}$, and $30 \mathrm{~cm}$ respectively (Figure 5.1.7). This neighbourhood averaging resampling algorithm was reported by Hay et al. (1997) to provide a better approximation of imagery acquired at a smaller scale than the more common nearest neighbour, bilinear, and cubic convolution resampling methods. 


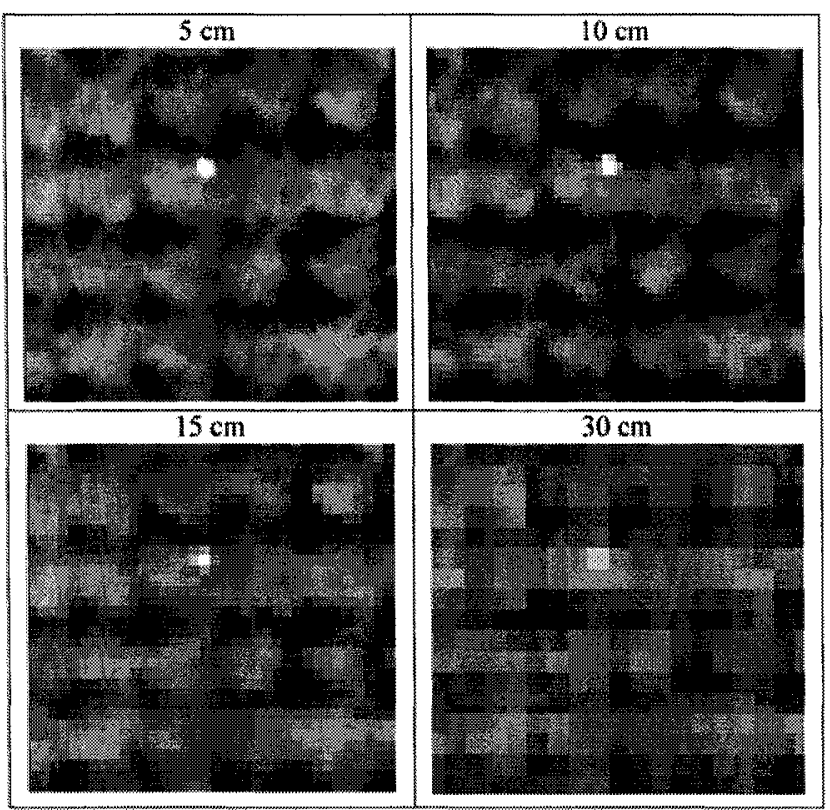

Figure 5.1.7: Example images showing the results of resampling to pixel spacings of $10 \mathrm{~cm}, 15 \mathrm{~cm}$, and 30 $\mathrm{cm}$ from the original $5 \mathrm{~cm}$ pixel spacing.

\subsubsection{Results}

\subsubsection{Tree Detection}

As expected, with fixed sized ordinary local maximum filters, increasing the sample window size increased omission error, whereas decreasing the window size increased commission error (Table 5.1.1). The LMRDA produced lower commission and omission error than all local maximum filters tested. Its overall accuracy was better in all cases but one $5 \mathrm{~cm}$ pixel spacing $20 \times 20$ local maximum filter, where it produced the same Accuracy Index. Errors of omission and commission for LMRDA were also more balanced, not varying as widely as the fixed window results. Thus, LMRDA provides accurate detection results and removes the difficulty of selecting the most appropriate sample window size for a local maximum filtering operation. The LMRDA detection 
results were not influenced by pixel spacing in the range of $5-15 \mathrm{~cm}$, whereas the much larger $30 \mathrm{~cm}$ pixel spacing did reduce detection accuracy significantly. For visual evaluation, Figure 5.1 .8 shows the $5 \mathrm{~cm}$ pixel spacing results overlaid on the original imagery. A large amount of the detection error for LMRDA was due to the presence of short ground vegetation in the imagery. It had a similar spectral response to that of the conifer trees, because it had started to become green at the time of imaging. In some cases, this led to its detection as an apex instead of an adjacent tree. The presence of short ground vegetation is a difficult problem, as it is hard to mask in multispectral imagery with broad spectral bands. Several classification attempts were made using grey-level cooccurrence texture measures along with spectral data in a maximum likelihood classification, but it was not possible to effectively separate it from the smaller conifer trees. A practical solution is true leaf-off imaging, just after snowmelt when only the conifers are green.

Table 5.1.1: Results of tree detection for several ordinary local maximum filters with fixed window sizes $(15 \times 15$ to $30 \times 30$ pixels $)$ and for the LMRDA at each of the pixel spacings studied $(5$ to $30 \mathrm{~cm})$.

\begin{tabular}{|c|c|c|c|c|c|}
\hline $5 \mathrm{~cm}$ Pixel Spacing & $15 \times 15$ & $20 \times 20$ & $25 \times 25$ & $30 \times 30$ & LMRDA \\
\hline Commission $\%$ & 33.3 & 10.1 & 6.1 & 2.5 & 10.1 \\
\hline Omission \% & 1.0 & 1.0 & 15.7 & 39.9 & 1.0 \\
\hline Accuracy Index \% & 65.7 & 88.9 & 78.3 & 57.6 & 88.9 \\
\hline $10 \mathrm{~cm}$ Pixel Spacing & $7 \times 7$ & $10 \times 10$ & $13 \times 13$ & $16 \times 16$ & LMRDA \\
\hline Commission $\%$ & 54.5 & 13.6 & 7.6 & 2.5 & 9.1 \\
\hline Omission $\%$ & 1.0 & 3.0 & 17.2 & 43.9 & 2.0 \\
\hline Accuracy Index \% & 44.4 & 83.3 & 75.3 & 53.5 & 88.9 \\
\hline $15 \mathrm{~cm}$ Pixel Spacing & $5 \times 5$ & $7 \times 7$ & $9 \times 9$ & $11 \times 11$ & LMRDA \\
\hline Commission $\%$ & 38.4 & 9.1 & 2.5 & 0.0 & 3.0 \\
\hline Omission $\%$ & 2.0 & 5.1 & 22.7 & 40.9 & 6.1 \\
\hline Accuracy Index \% & 59.6 & 85.9 & 74.7 & 59.1 & 90.9 \\
\hline $30 \mathrm{~cm}$ Pixel Spacing & $3 \times 3$ & $4 \times 4$ & $5 \times 5$ & $6 \times 6$ & LMRDA \\
\hline Commission $\%$ & 58.1 & 16.7 & 6.1 & 4.5 & 14.6 \\
\hline Omission \% & 2.5 & 9.6 & 31.8 & 50.0 & 5.1 \\
\hline Accuracy Index \% & 39.4 & 73.7 & 62.1 & 45.5 & 80.3 \\
\hline
\end{tabular}




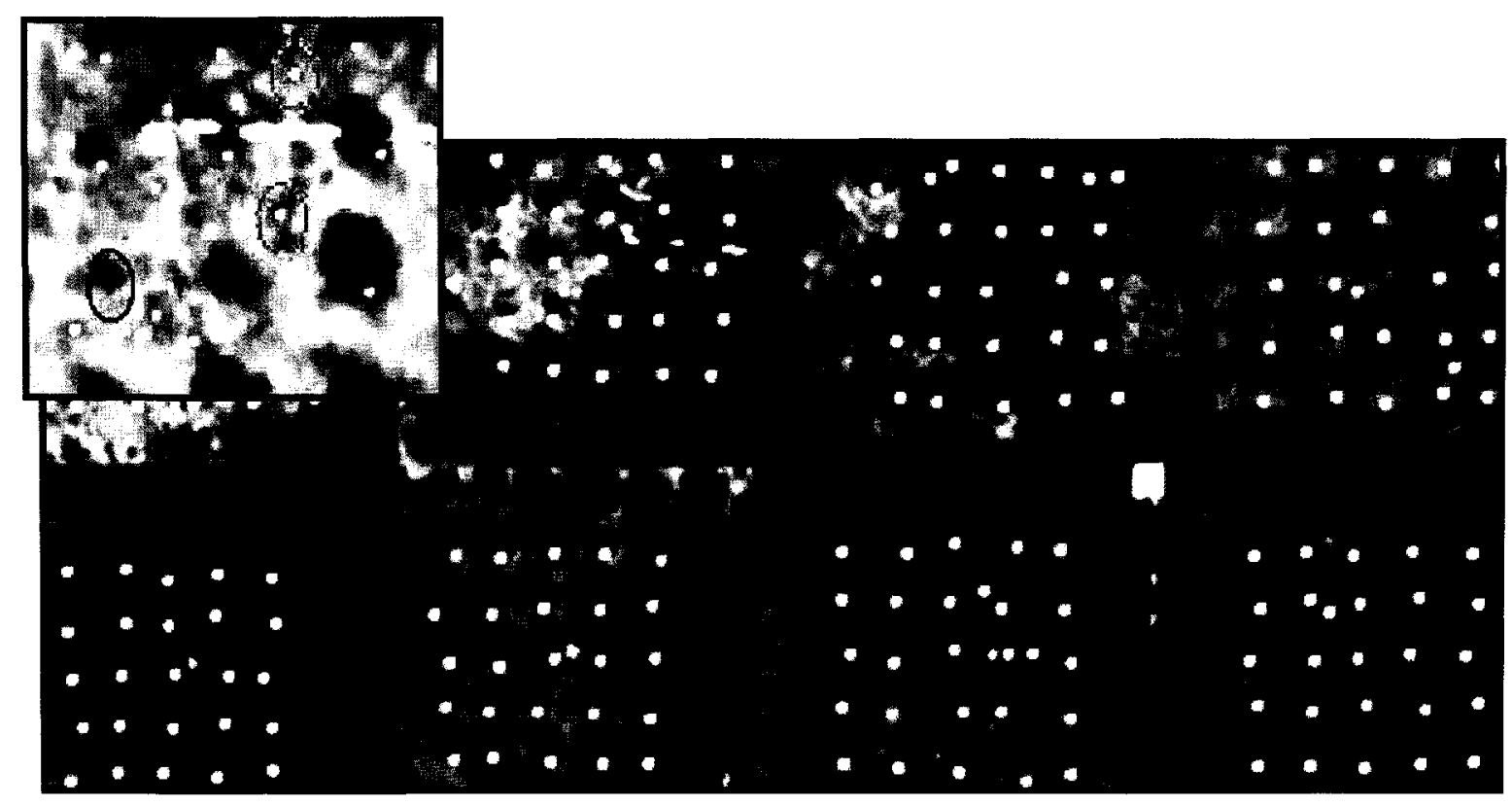

Figure 5.1.8: Detection results using $5 \mathrm{~cm}$ pixel spacing imagery. The outer row and column of trees in each plot were not included in the analysis because ground diameters had not been measured. In the zoomed in inset, cyan $=$ commission error due to the presence of short ground vegetation and red $=$ omission error.

\subsubsection{Tree Delineation}

Diameters determined from manual delineation best matched the ground measurements with approximately $11.2 \%$ error (Table 5.1.2). The automated method achieved a best result of $17.9 \%$ error for the $5 \mathrm{~cm}$ data. However, further testing with greater polygon angle removal $\left(40^{\circ}\right)$ produced a best automated delineation result of $14.5 \%$ with the smallest pixel spacing. In this case, using the higher angle removal resulted in very generalized crown shapes (more circular) whose measured diameters better matched those measured on the ground. In the field, crown diameter measured at the crown base did not include single long branches protruding from the crown or sharp indentations. It was taken as the visually representative diameter at the E-W, N-S measurement locations to avoid introduction of measurement outliers. Thus, for this study, measurement of 
diameters from more generalized crown delineations better matched the field measurement technique. Decreasing image resolution also increased delineation error non-linearly. The difference between the $5 \mathrm{~cm}$ and $10 \mathrm{~cm}$ image delineation was not significant (Bonferroni multiple comparison test, $\mathrm{p}=1.00$ ), while increasing the pixel spacing to $15 \mathrm{~cm}$ resulted in greater overestimation of the crown diameters. At $30 \mathrm{~cm}$, delineation error became extreme (Figure 5.1.9). This is an expected result, as larger pixels tend to artificially increase image crown diameters due to the averaging of the spectral radiation over a larger areal unit. Figure 5.1.9B most clearly shows this effect where the $15 \mathrm{~cm}$ pixel delineation error follows the same trend as the $5 \mathrm{~cm}$, but is consistently larger throughout the diameter range. The results from the $15 \mathrm{~cm}$ and $30 \mathrm{~cm}$ resolution delineations were significantly different from those using $5 \mathrm{~cm}$ imagery $(\mathrm{p}=$ $0.01, \mathrm{p}=0.00$, respectively). The $10 \mathrm{~cm}, 15 \mathrm{~cm}$, and $30 \mathrm{~cm}$ delineations were also significantly different from each other $(\mathrm{p}<0.03)$. Aggregating the individual tree results to average error for the study ("Mean Difference \%" in Table 5.1.2), substantially reduced error as over-estimates and under-estimates balanced each other in the averaging process. For the $5-15 \mathrm{~cm}$ pixel spacings, aggregation reduced error from $17.9-22.9 \%$ to 2.4-2.8\%. In addition, the average error of each of these automated delineations was less than that of the manual delineation.

Table 5.1.2: Error in average crown diameter measured from automated delineations at four image pixel spacings and for manual delineation at the $5 \mathrm{~cm}$ pixel spacing. The crown diameter to pixel size ratio (CD:PS) is shown beside each pixel size.

\begin{tabular}{|l|ccccc|}
\hline \begin{tabular}{c} 
Delineation\Error \\
\multicolumn{1}{|c|}{ Measure }
\end{tabular} & $\mathbf{5} \backslash 17: 1$ & $\mathbf{1 0} \backslash 8: 1$ & $\mathbf{1 5} \backslash 6: 1$ & $\mathbf{3 0} \backslash 3: 1$ & Manual 5\17:1 \\
\hline RMSE \% & 17.9 & 19.0 & 22.9 & 39.0 & 11.2 \\
Mean Difference \% & 2.8 & 2.8 & -2.4 & -28.4 & -4.1 \\
\hline
\end{tabular}

CD:PS - crown diameter to pixel size ratio. 

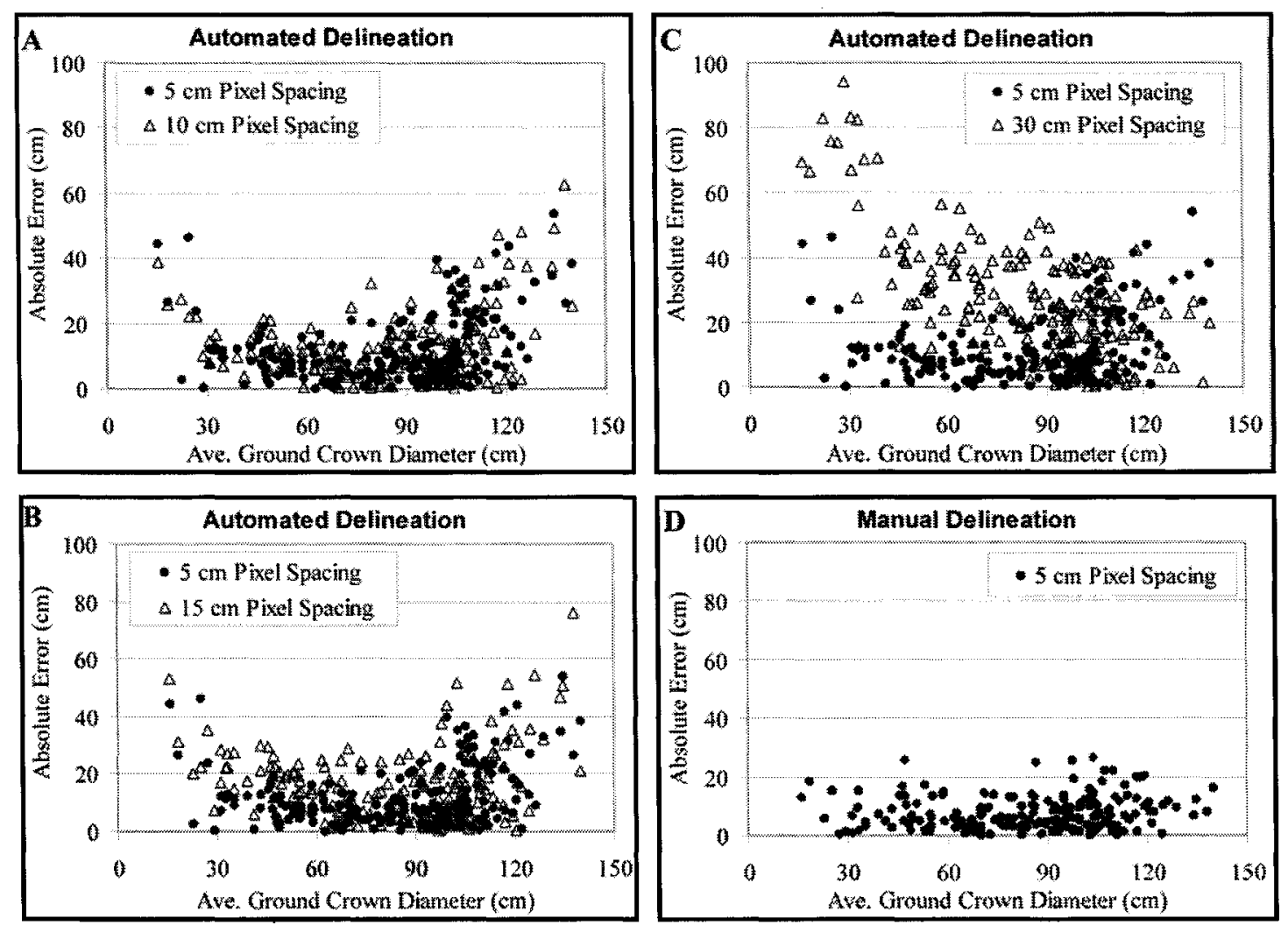

Figure 5.1.9: The relation between absolute error in crown diameter measured from LMRDA delineated crowns and ground measured crown diameter for $A .5 \mathrm{~cm}$ and $10 \mathrm{~cm}$ pixel spacings, showing little overall difference, B. $5 \mathrm{~cm}$ and $15 \mathrm{~cm}$ pixel spacings, showing similar trends but greater error over all diameters for the $15 \mathrm{~cm}$ data, C. $5 \mathrm{~cm}$ and $30 \mathrm{~cm}$ pixel spacings showing gross overestimation of small crowns in the $30 \mathrm{~cm}$ data, and $\mathrm{D}$. Manual delineation showing no apparent error trends.

For visual evaluation, the automated method using $5 \mathrm{~cm}$ pixel spacing and manual delineation results are presented in Figure 5.1.10. Comparison of the manual (cyan) and LMRDA (yellow) delineations by this means shows that plots with larger crowns (more crowded conditions identifiable in the imagery) resulted in the greatest disagreement between methods.

Examination of the individual errors revealed that the automated methods overestimated small and underestimated large crown diameters. Overestimation of the small crown 
diameters, similar to detection results, was due to the presence of short ground vegetation in close proximity to the crown boundary. In the case of large trees, errors were due to overlapping crowns, causing crown boundaries to be less distinct than the within crown spectral variance. As the image resolution was degraded, the within crown variance was reduced leading to improved delineation of larger crowns (Figure 5.1.9C). However, at the largest pixel size of $30 \mathrm{~cm}$, small crown boundaries were heavily distorted resulting in extreme overestimation of their diameters. Manual delineations did not suffer from any apparent estimation bias, producing a consistently low error across the range of diameters tested (Figure 5.1.9D).

Based on Figure 5.1.9A, it appears that the algorithm is most accurate within a range of crown diameters of $30-100 \mathrm{~cm}$ assuming a spatial resolution of $5 \mathrm{~cm}-10 \mathrm{~cm}$. This represents $63 \%$ of the originally sampled trees. Using observed values within this range improved the individual delineation results (Table 5.1.3). The largest improvement in accuracy was for the $5 \mathrm{~cm}$ pixel spacing reducing error from $17.9 \%$ to $14.0 \%$. However, as resolution degraded, the improvement decreased or resulted in greater error than that of the full range, suggesting that the $30-100 \mathrm{~cm}$ crown diameter range was most suited to the $5 \mathrm{~cm}$ pixel spacing (15:1 average crown diameter to pixel ratio). The manual delineation remained approximately the same, as the crowns were well delineated over the full crown diameter range and the manual interpreter was better able to identify the crown edge in the presence of short ground vegetation. Contrary to the full diameter range evaluation, the aggregated mean difference values for the delineations are noticeably different. For the reduced diameter range, all delineations overestimate the 
ground measured crown diameters. The smaller mean difference values presented earlier are due to the canceling effect of the over and underestimation of small and large crowns outside the optimal range examined here. Reducing the range of diameter sizes removed the cancellation effect revealing this trend.

Table 5.1.3: Delineation accuracy for crown diameter range of 30-100 $\mathrm{cm}$ with outliers caused by short ground vegetation removed. The crown diameter to pixel size ratios have been modified from Table 2 to reflect the data used in this subsample.

\begin{tabular}{|l|ccccc|}
\hline $\begin{array}{c}\text { Delineation/ Error } \\
\text { Measure }\end{array}$ & $\mathbf{5} / 15: 1$ & $\mathbf{1 0} / 7: 1$ & $\mathbf{1 5} / 5: 1$ & $\mathbf{3 0} / 2: 1$ & Manual 5/15:1 \\
\hline RMSE \% & 14.0 & 15.8 & 21.5 & 47.4 & 11.7 \\
Mean Difference \% & -3.1 & -4.9 & -12.9 & -39.8 & -6.0 \\
\hline
\end{tabular}

$\mathrm{CD}: \mathrm{PS}-$ crown diameter to pixel size ratio

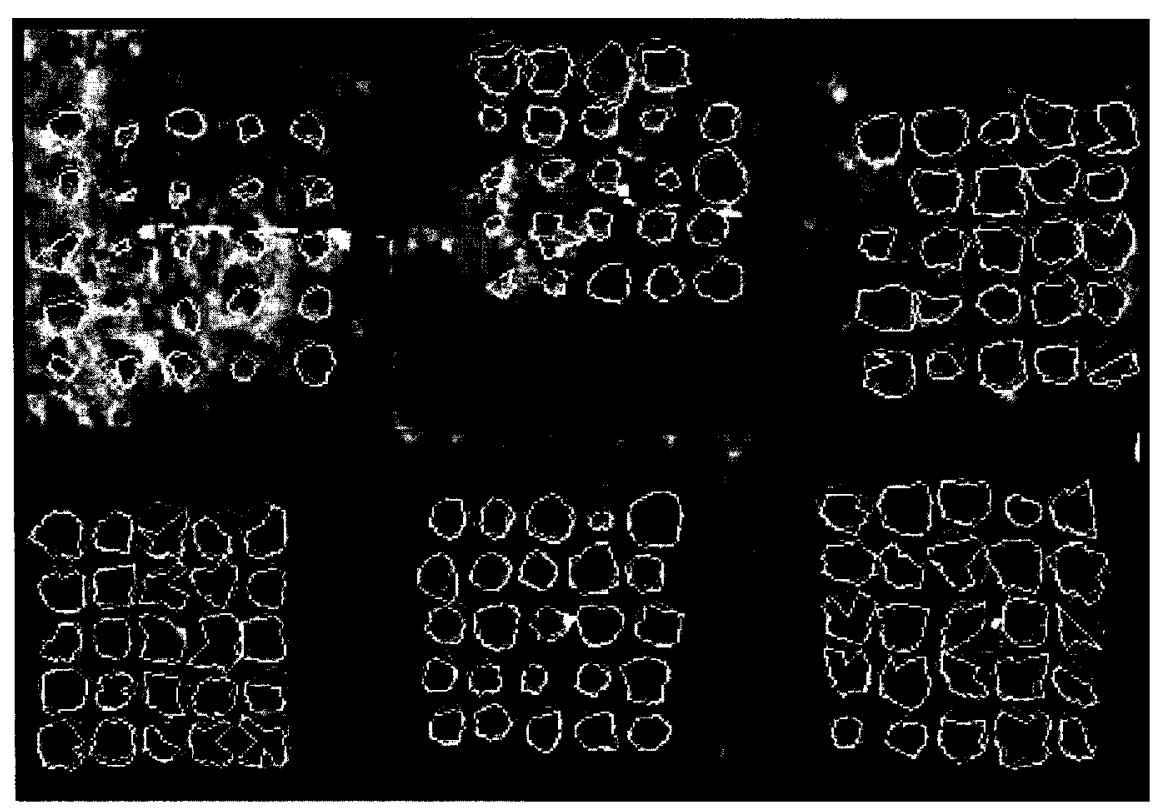

Figure 5.1.10: Example delineation results of LMRDA (yellow) and the manual method (cyan) using $5 \mathrm{~cm}$ pixel spacing. 


\subsubsection{Discussion}

The LMRDA produced better tree detection accuracies than the fixed window local maximum filters in all cases but one where they were equivalent. Detection accuracy was high, to within $90 \%$, for the well-controlled conditions studied. Results were also invariant to small changes in pixel spacing $(5-15 \mathrm{~cm})$, but larger pixel spacing caused significantly greater error. Crown delineation was more sensitive to changes in pixel spacing than crown detection. The smallest pixel spacing $(5 \mathrm{~cm})$ produced the most accurate diameter estimates $(17.9 \%$ error), while larger spacings increased delineation error in a non-linear manner. The most critical factor found to affect the delineation algorithm performance was the relative distinctiveness of the crown boundary in relation to within crown variation. If within crown variation is high and crowns are not overlapping, accurate results are likely to be obtained because the crown and background will be the most significant edge in the transect. However, in overlapping conditions, the within crown variability can be more distinct than the boundaries, causing delineation error. In this case, the solution is to reduce within crown variation. This requires estimation of the optimal image pixel spacing, as larger spacings reduce within crown variation, but also decrease the distinct spatial gradient used in delineation. Heuristic estimation of optimal pixel size for delineation can be based on previous studies in which crown size to pixel ratios have been calculated. In this study, the optimum average crown size to pixel ratio was found to be $15: 1$. Ratios below 15:1 (fewer pixels per crown) did not improve results as the within crown variability remained stable, but the crown boundary distinctiveness was reduced, leading to greater error. Ratios larger than 15:1 
(more pixels per crown) were heavily influenced by within crown variation, also resulting in substantial error.

For some forest management decisions, aggregated diameter measurements are required for a given management unit. In this study, aggregating individually measured crown diameters to the mean diameter of the sampled trees for the whole study reduced the error between automated and ground measured crown diameters to less than $3 \%$. Such areal averages may be useful as part of an initial flagging system to identify areas that are unlikely to meet future free-to-grow status, or in stocking estimation, although species composition must typically first be determined.

An important advantage of tree delineation is the potential to model tree structural variables such as tree height, stem diameter and biomass. In this study, preliminary results of LMRDA delineated crown diameters showed strong relations with tree height $(\mathrm{r}=0.86)$, stem diameter $(\mathrm{r}=0.78)$, and biomass $(\mathrm{r}=0.97)$ suggesting the potential to estimate these values using image based crown diameter estimates. On an individual assessment level it is unlikely that sufficiently accurate models could be obtained for prediction, but aggregating individual results from models could lead to reasonable areabased tree structural estimates useful in evaluating the growth success of crop trees.

The primary error sources in crown delineation that were identified in this study were crown overlap and the presence of short ground vegetation. The LMRDA algorithm produced good results even when crown overlap was significant, but errors did increase 
with increasing overlap. One reason for this is that the reference data consisted of diameters measured at the crown base while the delineated diameters were measured in imagery taken from above. As crown overlap increases, the delineated crown edge in the area of overlap is at progressively higher positions in the crown and not at the base. Thus, there is a greater mismatch of these two data types with increasing overlap. Crown overlaps in this study were also greater than would be expected in a 6-year old plantation because the well-controlled $1 \mathrm{~m}$ tree spacing was closer than typical operational spacings of $1.5-2 \mathrm{~m}$. Thus, the algorithm can probably provide similar results to those presented in this paper for plantations up to approximately 8-10 years old. In naturally regenerated forests or planted forests, a more random spacing will occur with greater variability in crown sizes and crown overlap. This could lead to detection-delineation error if crown size variability is too large to be encompassed by a single optimal pixel spacing. Evaluation and modification of the proposed algorithm to natural regeneration conditions will be an important aspect of future work, as only a small portion (maximum $18 \%$ ) of forest regeneration resulting from fire and harvesting consists of plantations (Natural Resources Canada, 2000).

The presence of short ground vegetation in the imagery presented problems for both the detection and delineation algorithms due to its similarity in spectral response to tree crowns for the spectral bands analyzed. Short ground vegetation re-establishes itself quickly after snow melt, making it difficult to acquire images without its presence. Masking the response of short ground vegetation may require the development of an explicit algorithm to examine imagery for crown and shadow components as short ground 
vegetation does not have an associated shadow. Alternatively, experimentation with advanced classification methods incorporating image textural characteristics may also be useful.

Another important element to evaluate, that was not present in this study, is the effect of competing vegetation. If the presence of overtopping competition strongly distorts the key spectral and spatial characteristics used in detecting and delineating crop tree crowns, then automated approaches may be inappropriate for assessing such regeneration conditions with remotely sensed data. This may occur, even in leaf-off conditions, for woody competition species such as willow, birch, aspen, and alder where dense branching may obscure the underlying conifer crown. Some herbaceous species such as raspberry and fireweed may also impact delineation results. After snowmelt, residual stems of these species still retain a vertical structure that could interfere with the spectral response of lower crop tree branches inhibiting the delineation process. Evaluation of this effect was not conducted at this stage of the research because the required subplots did not occur within 7 degrees of nadir making it difficult to determine if detection $\backslash$ delineation error was due to competition or view angle effects. However, further research is planned to develop a view angle correction to provide for this analysis.

The algorithm presented in this study has not yet been rigorously evaluated against other existing algorithms. Most have been developed and applied in mature forests with smaller crown diameter to pixel size ratios. The LMRDA algorithm was designed to utilize the high spatial information in large crown to pixel size (i.e., very high resolution) 
imagery. Conceptually, comparison with the most commonly reported technique, valley following, reveals some advantages of LMRDA in such imagery. Both approaches require searching for local maximum or minimum brightness values in an image using a moving window strategy. However, in tree detection, LMRDA re-examines identified local maximum values using a secondary reference window based on the surrounding spectral $\backslash$ spatial properties of a potential tree. Thus, if several local maxima are found in a single crown, only one should remain to represent the crown position. In valley following, the local minima used for delineation and subsequent apex detection are subject to greater error with high-resolution imagery due to the increased presence of local within crown valleys that can result in multiple image segments being created for a single crown. A refinement procedure, such as that presented here, to ensure that only appropriate local minimum values are used in boundary delineation may help to alleviate this problem. In crown delineation with the high-resolution imagery of this study, crown boundary detection using local minimum values tended to overestimate crown edges while the position of maximum rate of change used in LMRDA was more accurate. Thus, valley following may be more suited to coarser resolution imagery and larger trees (crown diameter to pixel size ratios ranging from $3: 1$ to $8: 1$ ) where crown boundaries are often effectively represented by local minimum values. However, given that delineation can currently only be accomplished with a spatial precision of one pixel (until sub pixel algorithms are developed), valley following delineation of small trees such as those of this study using coarser resolution imagery, would likely result in significant relative error. High-resolution imagery also provides greater within crown data for spectral and textural evaluation of crown condition. Further research will continue these comparative 
evaluations to aid in refinement of the algorithm and to provide more quantitative comparisons with other methods.

\subsubsection{Conclusion}

A tree detection-delineation methodology was developed for high-resolution remotely sensed imagery. It was successfully applied in leaf-off $5-15 \mathrm{~cm}$ pixel imagery of a sixyear-old regenerating forest plantation. Individual tree detection accuracy produced a best result of $91 \%$. Delineated tree crown diameters were within $17.9 \%$ of field measurements of crown base diameters on an individual tree basis and within $3 \%$ when averaged for the study. Further research will include evaluation of view angle and vegetative competition effects on detection-delineation accuracy, and application of the algorithm in an operational cutover. These methods and additional knowledge will be integrated into a remote sensing-based methodology for free-to-grow assessment.

\subsubsection{Acknowledgements}

This research was funded by grants to D. King from the Natural Sciences and Engineering Research Council of Canada and the US National Geographic Society. The Ontario Forest Research Institute and the Canadian Forest Service provided support for airborne and field data acquisition and valuable advice. Images used in the study were acquired by the Ontario Ministry of Natural Resources Data Acquisitions Branch. 


\title{
5.2 DETERMING AN OPTIMAL APPROACH FOR AUTOMATED TREE DETECTION
}

Citation: Pouliot, D.A., and D.J. King (2005a). Approaches for optimal automated individual tree crown detection in young regenerating coniferous forests. Canadian Journal of Remote Sensing, 31: 255-267.

\begin{abstract}
Automated tree detection provides a means to acquire information on tree abundance and spatial distribution, both critical for evaluating the status of regenerating forests. It is also often a pre-cursor to automated tree delineation, which typically utilizes image data surrounding a detected crown point. However, obtaining consistently accurate detection results has proven difficult due to errors associated with image scale. In this paper, four approaches that reduce this scale dependence are evaluated, including determination of (1) optimum global and (2) optimum local image smoothing to apply pre-detection, (3) determination of the optimal local window size for use in the detection algorithm, and (4) post detection merging of initially defined crown segments. Each was applied to three datasets acquired by different sensors and with different regenerating forest conditions. A common local maximum tree detection algorithm was implemented for approaches 13, while a watershed segmentation algorithm was applied in approach 4 . Detection accuracy was evaluated using standardized methods. The highest accuracies for each dataset were obtained with approaches based on local scale representations where the regenerating structure favored such approaches. However, more consistent accuracies
\end{abstract}


across all datasets were obtained with the optimum global scale approach. Post detection merging of adjacent crown segments produced the poorest results. Error sources and the advantages and disadvantages of each approach are discussed in terms of developing more operational methods for automated tree detection in regenerating forests.

\subsubsection{Introduction}

In regenerating forests, accurate knowledge of tree abundance and spatial distribution is important in predicting future forest conditions and to determine if these conditions are sufficient to meet the preset management objectives for the site (Pitt et al. 1997). High spatial resolution remote sensing provides a potentially low cost means to obtain this information if efficient and reliable methods for detection and measurement of individual trees in imagery can be developed. Automated individual tree detection algorithms have been developed for this task, but accuracy has proven to be highly dependent on scale making consistent results difficult to achieve (Gougeon, 1995a; Dralle and Rudemo, 1996; Brandtberg and Walter, 1998; Gougeon and Leckie, 1999; Pinz, 1999; Walsworth and King, 1999; Wulder et al., 2000; Culvenor, 2000; Pitkanen, 2001; Pouliot et al., 2002; Erikson, 2003). Most algorithms, with the exception of Gougeon and Leckie (1999) and Pouliot et al. (2002) have been tested in mature forest conditions. However, common to these studies has been a noted difficulty of defining a scale representation in a consistent manner to achieve optimal detection results. Here, the term 'scale' refers to the 'grain' or detail of an image and not the extent or coverage. 
Considering an image with a globally defined scale, small trees relative to the scale are often not detected (omission error) due to their weak spectral and spatial response in the imagery, whereas larger trees may cause false detection (commission error) due to crown branches being detected as single tree crowns. In image acquisition, a common approach is to acquire imagery with a pixel size suitable to detect the smallest required object (i.e., using a criterion such as $1 / 2$ the object size), as this minimizes omission error. However, this can lead to high commission errors in larger crowns due to within crown branch clusters being detected as single crowns and due to image noise. An 'optimal' global scale is defined as that which minimizes both errors of omission and commission, producing the best overall detection results. An alternative approach is to find the optimum local scale for each object of interest (i.e. trees). Optimum local scale is not defined for a minimized error, but is the scale where no error exists. That is, the appropriate local scale can be defined so that the tree is detected as a single object.

The scale of an image can be modified in several ways; image smoothing is most commonly applied, with the appropriate smoothing factor or window sample size criteria being difficult to define for optimal tree detection. Thresholds or parameters can also be considered as scale modifications when linked directly to object size or indirectly to size through an image property that is size related. For example, the variance threshold in a region growing algorithm indirectly controls the size of the region found.

The scale component of automated tree detection analysis has only been addressed in a few studies concerned with mature forests. Brandtberg and Walter (1998) made use of 
scale space theory to constrain local maximum detection based on multiscale edge detection in scanned colour infrared photography and more recently for local based scale optimization in lidar imagery (Brandtberg et al., 2003). The scale space approach involves examination of changes in image brightness with changes in scale simulated by a Gaussian smoothing function (Lindeberg, 1994). Its advantage is that it does not require any prior information regarding the objects to be extracted. However, the number of objects to be extracted from the set of scale space objects and the scale interval and range must be selected and are typically determined arbitrarily or empirically using field data. Further, high contrast objects are likely to be found as significant objects regardless of whether they represent actual image features of interest (Hay et al., 2003). In cases where prior knowledge exists about the objects of interest, improved object extraction and simplification of the scale selection process is possible. For example, Wulder et al. (2000) exploited the spatially dependent nature of tree crowns using semivariance analysis to estimate the optimal window size for local maximum filtering, but found that using local edge detection produced better window size estimates. Pouliot et al. (2002) also found that the latter approach improved tree detection results in young regenerating forests. These optimal window size approaches can be effective, but are not ideal as only one local maximum is returned within the window. For larger windows, more than one maximum may exist, representing several trees, resulting in omission errors. In a similar approach, Pinz (1999) used the mean brightness value in successively larger sample windows to evaluate whether a given local maximum was likely to represent a tree crown. This approach removes error associated with large windows containing multiple crowns, but how the evaluation was performed and its effects on the final results were 
unclear. Culvenor (2000) proposed a simple method analogous to wavelet analysis to determine the optimal global scale. It is based on an examination of the number of local maxima detected at different levels of scale simulated using a Gaussian filter, followed by selection of the optimum global scale as the point at which the rate of change of the second derivative is a maximum. In lidar data, Persson et al. (2002) used local surface fitting to determine which of three user defined scales was optimal for detection of a given tree crown.

Each of these approaches has been found to improve results in their intended applications. However, comparison and adaptation of these methods to other studies is difficult for several reasons. First, a wide range of accuracy evaluation methods are applied and therefore results are not directly comparable. Second, methods have been developed and tested with specific image formats and forests conditions making it difficult to evaluate their dataset dependence. Third, methods vary in terms of required parameters and the sensitivity of tree detection to these parameters. This makes it difficult to determine how precisely such parameters must be specified and how easily this precision can be achieved. Finally, the methods are often presented as part of a larger study involving automated detection and delineation, and in some cases, the methods used to optimize tree detection are not well described.

The purpose of this study was to determine the best approach for automated tree crown detection in regenerating forests. The issues identified above are explicitly addressed in selection of data types and analytical procedures. Four approaches are compared that 
integrate methods previously presented in the literature with new methods. They are evaluated in terms of their accuracy using standard methods, their consistency across a range of data types and regeneration conditions, and their input parameter dependence. The four approaches are: 1) the local maxima scale relation described by Culvenor $(2000), 2)$ the variable window size method described in Wulder et al. (2000) and modified by Pouliot et al. (2002), 3) a new approach for local image smoothing based on variable window size methods, and 4) a new approach based on post detection merging of detected segments. The first three are applied pre-detection to identify an optimal representation of scale for local maximum based detection analysis. The last approach involves evaluation of detection and initial crown segmentation results to identify segments that should be merged to represent a single crown.

\subsubsection{Datasets}

Three subsets of larger datasets were used consisting of a range of variability in regeneration tree structure that would be typical of operational applications. The datasets consisted of an experimental study with highly controlled conditions, a young regenerating cutover, and an older regenerating cutover. The latter two were from operational forest management areas. As each data set was from separate and larger studies of the Ontario Ministry of Natural Resources (OMNR), the Canadian Forest Service (CFS), and Carleton University, image types and pixel sizes varied. Specific characteristics of each dataset are listed in Table 5.2.1. Example images from each dataset are shown in Figure 5.2.1. All imagery was acquired before deciduous and 
herbaceous vegetation leaf flush to minimize commission error from background vegetation.

Table 5.2.1: Summary of selected characteristics for datasets used in the study.

\begin{tabular}{|c|c|c|c|c|c|c|c|c|c|c|}
\hline $\begin{array}{l}\text { Study } \\
\text { Site }\end{array}$ & $\begin{array}{c}\text { Pixel } \\
\text { size } \\
(\mathrm{cm})\end{array}$ & $\begin{array}{c}\text { View } \\
\text { Angle } \\
\text { (degrees) }\end{array}$ & $\begin{array}{c}\# \\
\text { Trees }\end{array}$ & $\begin{array}{c}\text { Dominant } \\
\text { Species }\end{array}$ & $\begin{array}{c}\text { Age } \\
\text { (years) }\end{array}$ & \multicolumn{3}{|c|}{ Crown Diameter $(\mathrm{cm})$} & $\begin{array}{c}\text { Average } \\
\text { Height } \\
\text { (cm) }\end{array}$ & $\begin{array}{c}\text { Density } \\
\text { (trees ha } \\
1)\end{array}$ \\
\hline ESS & 5 & 7 & 197 & Spruce & 6 & 85 & 27 & $16-145$ & 127 & 10000 \\
\hline $\mathrm{YCO}$ & 6 & 10 & 265 & $\begin{array}{l}\text { Spruce, } \\
\text { Pine }\end{array}$ & 5 & 58 & 31 & $5-135$ & 110 & 3155 \\
\hline $\mathrm{OCO}$ & 15 & 25 & 144 & Spruce & 15 & 129 & 43 & $40-230$ & $>200$ & 2300 \\
\hline
\end{tabular}

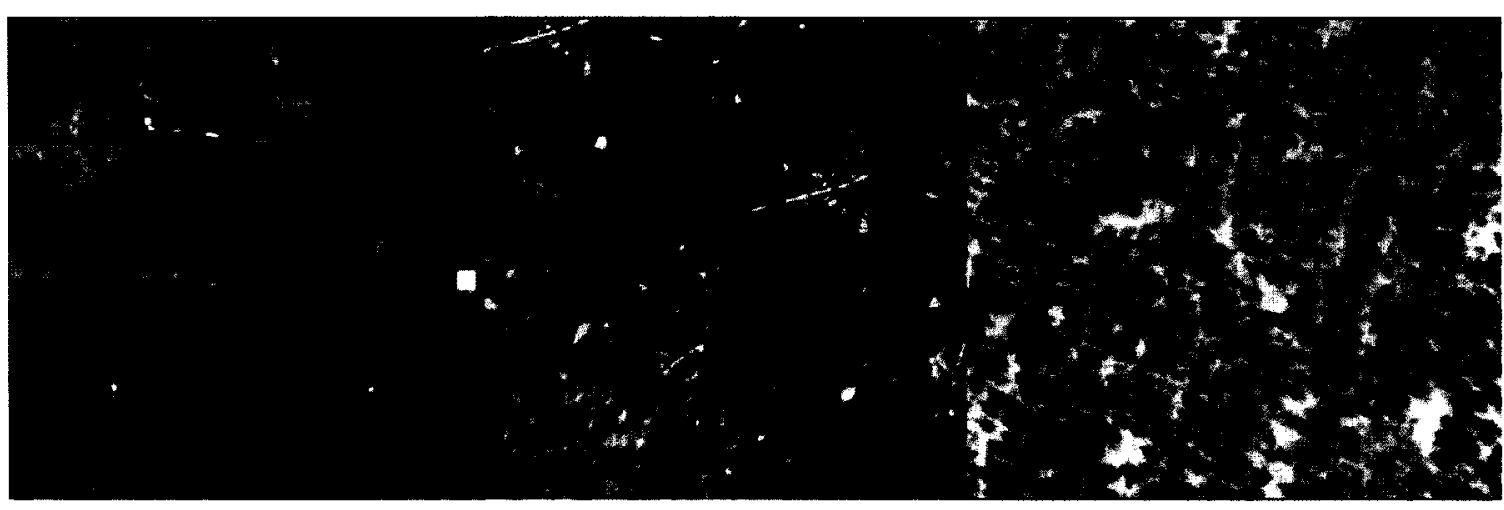

Figure 5.2.1: Example images of datasets used in the study. Left - experimental study site (ESS), middle young cutover (YCO), right - older cutover (OCO).

\subsubsection{Experimental Study Site (ESS)}

This study area was located outside Sault Ste. Marie, Ontario $\left(46^{\circ} 33^{\prime} \mathrm{N}, 84^{\circ} 27^{\prime} \mathrm{W}\right)$. It was compiled from an experimental study site established in 1994 by the OMNR and CFS as part of an effort to identify the effects of various levels of vegetative competition on black spruce (Picea mariana) and jack pine (Pinus banksiana) crop trees (Bell et al., 2000). Trees were planted at a spacing of $1 \mathrm{~m}$ in $7 \times 7 \mathrm{~m}$ plots with different species and intensities of competing vegetation. For this study, only plots containing black spruce trees were used because by 2000 , the jack pine crowns had grown into one another in 
such a way that individual detection was not possible. Plots with competing vegetation were also excluded in order to remove their effects on automated detection.

Images of the study site were acquired in a leaf-off condition on April 25, 2000 between 12:00 - 14:30 using a Kodak DCS 460 CIR digital camera with $\sim 5 \mathrm{~cm}$ nominal ground pixel size. Three spectral bands were acquired in the green $(500-600 \mathrm{~nm})$, red (570-780 $\mathrm{nm})$, and near infrared (710-800 nm). The camera uses a single charged coupled device (CCD) with a filter corresponding to the selected wavelengths placed over the photosites in a Bayer pattern. As each photosite receives radiance from one spectral band, interpolation is used to derive data for the other two bands.

For validation of detection results, tree positional data were not required, as the initial planting arrangement of $1 \mathrm{~m}$ spacing was known and was clearly identifiable in the imagery.

\subsubsection{Young Cutover (YCO)}

This dataset represented young regeneration conditions occurring after harvesting and planting. It was located northeast of Sioux Lookout, Ontario $\left(50^{\circ} 18^{\prime} \mathrm{N}, 91^{\circ} 39^{\prime} \mathrm{W}\right)$. Black spruce and jack pine were planted in 1997, but most of the pine was natural infill. Competition consisted of pockets of high-dénsity aspen (Populus tremuloides) and lower density white birch (Betula papyrifera). The aspen tended to be considerably taller than the birch, and both aspen and birch often overtopped the spruce and pine trees. The topography was hilly with gentle slopes. 
Image data were acquired on May 11, 2002 between 11:30 - 15:00 using a DuncanTech MS3100 CIR digital camera. It employs beam splitting technology to focus incoming light on 3 CCDs and capture separate wavelengths simultaneously. This allows it to produce higher spectral quality image data (i.e. non-overlapping bandwidths and less correlated bands) than can be acquired using single CCD cameras such as the Kodak DCS. Images were acquired in the green $(500-600 \mathrm{~nm})$, red $(600-700 \mathrm{~nm})$, and near infrared $(700-900 \mathrm{~nm})$ with $\sim 6 \mathrm{~cm}$ nominal ground pixel size.

For validation, circular plots with a radius of $3 \mathrm{~m}$ were laid out in the field and the plot centre marked using a $40 \times 40 \mathrm{~cm}$ white board set out before imagery was acquired. The direction and distance of each conifer tree within the plot to the plot centre was measured. These data were used in a GIS to create a reference map of crown locations.

\subsubsection{Old Cutover (OCO)}

This study site was located southwest of Thunder Bay, Ontario $\left(45^{\circ} 10^{\prime} \mathrm{N}, 89^{\circ} 50^{\prime} \mathrm{W}\right)$ representing older regeneration conditions occurring after harvesting and planting. It was part of the OMNR Fallingsnow Ecosystem Project designed to evaluate the effects of chemical and mechanical vegetation competition on flora and fauna (Lautenschlager et al., 1997). White spruce (Picea glauca) was planted in 1988 but balsam fir (Abies balsamea), white pine (Pinus strobus), jack pine, and white cedar (Thuja occidentalis) had naturally infilled. Competition consisted mostly of aspen and alder (Alnus spp.). Aspen ranged from low to high density and was typically taller than conifers. Alder also 
ranged from low to high density, but tended to be of equal or lower height than conifers. The site had moderate to steeply sloped topography, however, data used in this study were comprised only of the moderately sloped areas.

Colour infrared photography was acquired at 1:5000 scale on May 21, 2003 between 11:00 and 15:00 using a Wild RC-10 aerial mapping camera with Kodak Aerochrome Infrared II 2443 film. The photographs were scanned at $850 \mathrm{dpi}$ to $15 \mathrm{~cm}$ nominal ground pixel size. These data represent typical operational imagery used in many forestry applications and were acquired as part of the Fallingsnow project to provide detailed maps of conifer and other vegetation cover for wildlife habitat and population studies.

For validation, seven 1:500 colour photographs were acquired and scanned at $400 \mathrm{dpi}$ to give a nominal ground pixel size of $3 \mathrm{~cm}$. Visual interpretation of these photographs was carried out by the first author for validation of detection accuracy with this dataset.

\subsubsection{Methods}

\subsubsection{Image Pre-Processing}

Before automated tree detection is performed, most studies have applied some means of initial separation of vegetation pixels from non-vegetation pixels. This serves to eliminate brightness gradients not associated with vegetation that could result in false detection of brightness peaks as trees. Thresholding is commonly employed for this task (Gougeon, 1995a; Gougeon and Leckie, 1999; Walsworth and King, 1999; Culvenor, 2000; Pitkanen, 2001; Pouliot et al., 2002; Persson et al., 2002; Erikson, 2003), but in this 
study supervised maximum likelihood classification of 5 classes (soil, water, snow, shadow, and vegetation) was used for the following reasons. First, vegetation pixels in the imagery were spectrally distinct from all other classes (minimum Bhattacharrya separability of 1.999), suggesting the potential for high classification accuracy. Second, because these classes were distinct, the classification results were less dependent on well defined training data, thus simplifying the classification task and increasing its repeatability. Third, statistical classifiers are less sensitive to image brightness variations such as that resulting from bidirectional reflectance or topography as the defined statistical criteria provide greater discretion in pixel class assignment compared to thresholding.

The near infrared band was used for automated detection in the ESS and YCO datasets while the green band was used for the OCO dataset as vegetation was saturated in some portions of its near infrared imagery. An initial $(1 \sigma)$ Gaussian filter was applied to reduce image noise and convert the data to floating point format so that local maxima were represented by a single pixel. In byte format, local maxima often exist as groups of pixels due to the reduced precision. In very high resolution data such as that used in this study considerable pre-smoothing can be applied for noise removal without drastically impacting the image morphology. However, with coarser resolution imagery care must be taken as smoothing data may negatively affect detection accuracy.

Local maxima detection was carried out using a moving $3 \times 3$ window where a local maximum was identified only when the centre pixel in the window was greater than all 8 
of the surrounding neighbours. This is the most common method applied in tree detection studies; it was used for the three optimum image scale approaches described below. The fourth approach used a watershed segmentation method for local maxima detection. The detection results were the same using either method, but in the watershed method the local minima network surrounding a local maximum was also defined, providing an initial crude segmentation of the crowns.

\subsubsection{Approaches to Optimal Detection}

\subsection{Number of Local Maxima and Smoothing Relation (LMSR)}

This approach to optimal detection attempts to identify the optimal level of global smoothing to apply before local maxima detection is carried out. It is adopted from Culvenor (2000) who applied it to simulated imagery of mature eucalyptus forests. In this approach, the relation between the number of local maxima detected and the Gaussian smoothing factor is evaluated to identify the optimum. The Gaussian filter is given in equation 5.2.1:

$$
f(x)=\frac{1}{2 \pi \sigma^{2}} e^{-(x)^{2} / 2 \sigma^{2}}
$$

Where $x$ is the distance from the filter centre and $\sigma$ is the smoothing factor represented as the standard deviation in pixels. 
The local maxima smoothing relation is determined for the original image scale and for simulated scales derived using a range of $\sigma$ values. For the three datasets used in this study, $\sigma$ ranged from 0.1 to 4.0 in increments of 0.2 . The automated method given in Culvenor (2000), which determined the optimal scale as the maximum rate of change in the second derivative, proved to be highly sensitive to noise, giving inconsistent results. For this reason, the relation was visually assessed to determine the optimal smoothing factor. Referring to Figure 5.2.2, an example smoothing relation, the first step in the visual assessment is to plot the relation as the number of local maxima vs. smoothing factor. The next step is to determine the longest line that can be fit to the curve (plotted relation) starting with the last point on the right side of the graph and moving towards the first point on the left side while maintaining a suitable fit. This is defined as a linear fit where residuals are relatively small and equally distributed on either side of the line. The final step is to determine the point where this line and the curve start to diverge. This point is taken as an indication of the optimal smoothing factor required. Figure 5.2.2 shows the line that best fits the example smoothing relation and identifies the optimal Gaussian smoothing factor.

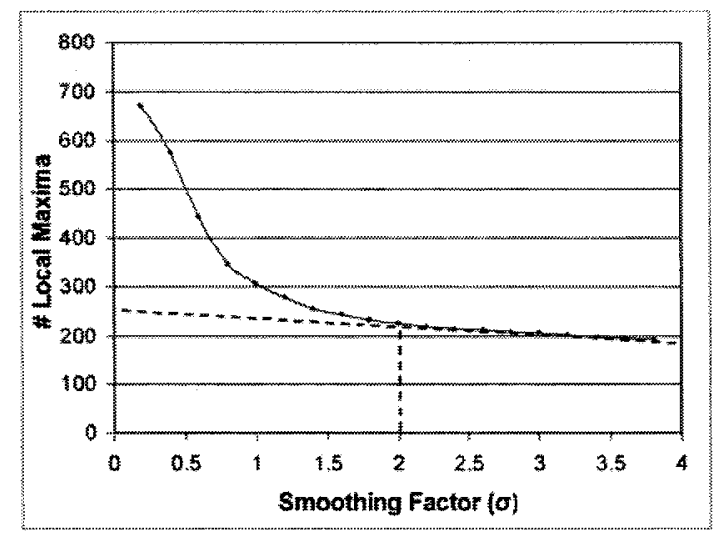

Figure 5.2.2: Solid line - example local maxima smoothing relation. Dashed line - indicates the visual rule used to determine the optimal Gaussian smoothing factor. 


\subsection{Variable Local Window Size (LWS)}

This approach is based on the method outlined in Pouliot et al. (2002), originally adapted from Wulder et al. (2000). In this approach initially detected local maxima are assessed using locally resized circular sample windows to determine final estimates of local maxima positions. The local window size is determined based on an estimate of spatial dependence. Tree crowns tend to be highly spatially dependent objects in images, therefore local estimates of spatial dependence can produce reasonable estimates of crown size (St-Onge and Cavayas, 1995; Hay et al., 1997; Levesque and King, 1999; Butson and King, 2001). To derive an approximate measure of the ratio of spatial dependence to crown size, local transects are extended outward from the local maxima being processed. The length of the transects are initially set to that of the largest expected crown in the imagery. Each transect is subsequently reduced using a 4th order polynomial by removing tail end transect data until a minimum acceptable $r^{2}$ value is obtained for the polynomial fit. This acts as a form of scaling, to help ensure that transect length is appropriate for the crown being processed. The strongest edge (largest value in the first derivative) within each reduced transect is taken as a measure of the crown radius in that direction and the mean for all transects is taken as the final crown radius estimate and used as the window size for local maxima detection. In this study, an initial transect sample distance of 15 pixels, with 36 transects separated by 10 degrees were used for each local maximum. Increasing the number of transects did not improve results. To scale transects, an $r^{2}$ threshold of 0.98 was used, but any value above 0.96 produced very similar results. These parameters were applied for all datasets. 


\subsection{Local Smoothing Factor (LSF)}

This approach was motivated by the success of the LWS methods identified in previous research (Pouliot et al., 2002) but with modifications to address its limited ability to detect local maxima of trees growing in close proximity in relation to the locally determined window size. The approach is essentially the same as the LWS approach, but instead of estimating window size, the amount of local smoothing required to appropriately detect the tree crown is estimated. Estimating the smoothing factor rather than a sample window reduces the probability of omission error because detection is carried out using the minimum window size of $3 \times 3$, while in the LWS approach windows can be any size. As in the LWS approach, initial local maxima are detected and reassessed using local transect analysis to estimate the crown size. The crown size estimate is converted to a Gaussian smoothing factor using an empirically derived equation. A continuous surface is created from the estimated smoothing factors using inverse distance weighting interpolation with a minimum of three points. This surface is then used to locally smooth the image by creating a unique Gaussian filter for each pixel based on the estimated smoothing value. Figure 5.2.3 shows an example image, the surface of estimated $\sigma$ smoothing factors, and the resulting locally smoothed image. To estimate the final tree locations, the $3 \times 3$ local maxima detection filter is passed over the locally smoothed image.

The equation used to estimate the smoothing factor from the local transect based crown size estimate was determined empirically using 15 crowns extracted from the imagery for each of the three datasets in the study. Approximately five crowns were selected in each 
of three size categories of small, medium and large. The amount of smoothing required to produce only one local maximum for each of these crowns was determined as well as the crown size estimate from the local transect edge detection method. Figure 5.2.4 shows the relation between crown size and Gaussian smoothing factor that was applied to force the crown to contain a single local maximum.

It was expected that this relation would be highly data dependent, requiring separate relations to be defined for each dataset. However, Figure 5.2.4 reveals that this dependence was not as strong as anticipated and suggests that a generalized equation could be used. Thus, a line was fit to the upper bound of the data for all datasets by modifying the slope and offset of an initial least squares fit to the data ('heuristic fit' in Figure 5.2.4). The equation for this line $(y=0.3 x-0.5)$ was used to determine the local smoothing factor. Apart from this equation the same parameter values used in the LWS approach were used.

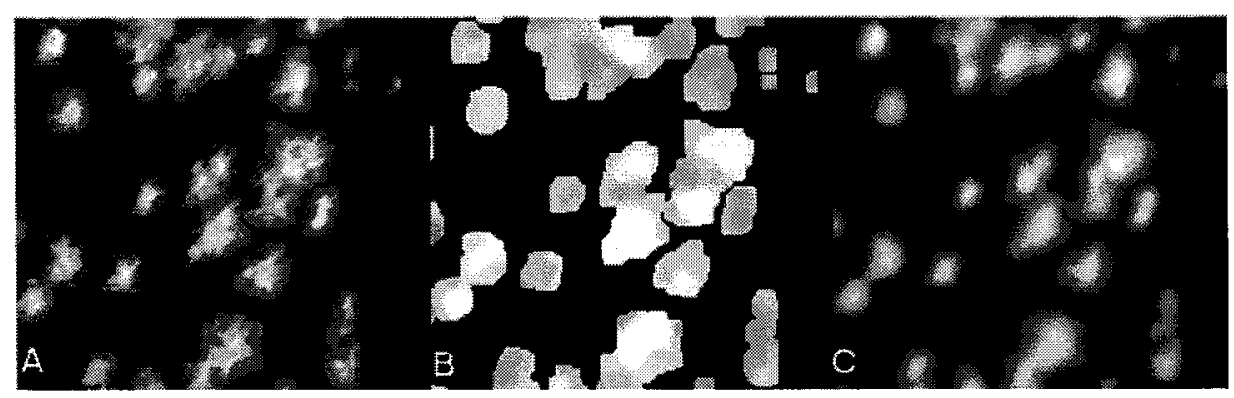

Figure 5.2.3: Example of (A) original image, (B) smoothing factor image, (C) locally smoothed image. 


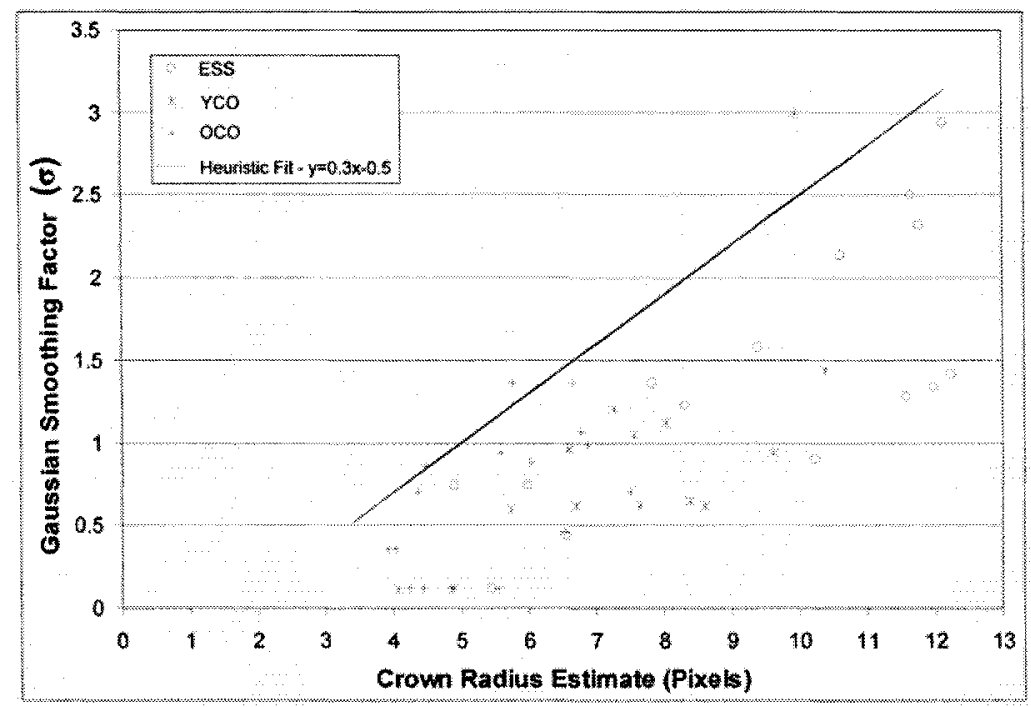

Figure 5.2.4. Relation between mean local edge estimate for a crown and degree of Gaussian smoothing required to detect only one local maximum for the crown object.

\subsection{Post Detection Merging (PDM)}

In this approach, the strategy is changed from optimally scaling data for input in automated detection processing to examining the initial detection results to make local improvements. The watershed segmentation method described in Persson et al. (2002) is used to estimate local maxima positions and the local minima surrounding each maximum, which crudely represent the boundary of the tree crown. The position of the boundary is directly determined by the initial thresholding or classification method used to extract crown pixels. It may not provide consistent crown boundary delineations due to brightness variations caused by bidirectional reflectance, optical effects, and topography. However, to aid detection, these boundaries can be useful. Referring to Figure 5.2.5, each boundary pixel common to two crown segments is examined to find the location of maximum brightness along the boundary referred to as the valley brightness (VB). The brightness values for the local maxima positions of the two crown segments being 
compared are also extracted (LM1 and LM2). The lower of these two values (LM2) is used to calculate the relative percent difference with the valley brightness. If the percent difference is less than a user-defined threshold, the two crown segments are merged. This process is iteratively applied until no further merging occurs. The selection of the difference threshold is subjective, but is the only required parameter in this approach. Tests with various data types and forest conditions could be used to construct a database or relation to provide reasonable estimates for this parameter. In this study, thresholds of $20 \%, 6 \%$ and $6 \%$ were used for the ESS, YCO, and OCO datasets, respectively.

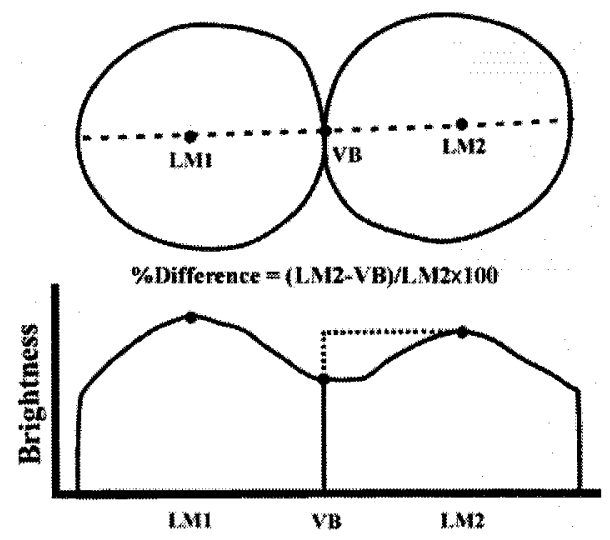

Figure 5.2.5: Adjacent crown segments (top) and profile of brightness values (bottom) from a given local maximum (LM1) through the maximum brightness value along the common boundary (VB) to the adjacent local maximum (LM2). The dotted line in the brightness profile identifies the features being compared to determine the merging decision.

\subsubsection{Evaluation of Detection Results}

Detection accuracy was evaluated using a method similar to that of Pitkanen (2001), where points from automated tree detection were assigned to ground measured reference locations based on an iterative search distance algorithm. In the first iteration, all detected tree points within a given search distance of a reference point were found, the closest was assigned as a match to the reference point and both points were removed from further 
consideration. In subsequent iterations, a larger search distance was used up to a maximum search distance of approximately $1 / 2$ the size of the average crown size in the dataset. In this study, search distances of $0.1 \mathrm{~m}, 0.2 \mathrm{~m}$ and $0.3 \mathrm{~m}$ were used. After the algorithm was complete, reference trees not matched to detected maxima were taken as omission errors, detected trees not matched to a reference tree were taken as commission errors, and matched points were considered correctly identified trees. The number of omission, commission, and correctly identified trees were reported along with an accuracy index that combines errors of omission and commission as shown in equation 5.2.2.

$$
A I=[(n-o-c) / n] \times 100
$$

Where $n$ is the actual number of trees that exist in the study area, $o$ is the number of omission errors and $c$ is the number of commission errors. Negative $A I$ values are possible and occur when commission and omission errors are greater than the total number of trees to be detected.

To provide a baseline for comparison of the four optimal detection approaches, the actual optimal global smoothing factor was determined empirically. Each image dataset was smoothed using factors from 1 to 3.5 in increments of $0.5 \sigma$, local maxima were detected using the $3 \times 3$ filtering method, and accuracy results were compiled as described above. The optimal factor was taken as that which produced the highest accuracy index. 


\subsubsection{Results and Discussion}

\subsubsection{Empirically Derived Optimal Smoothing}

For the empirical baseline analysis, the optimal global smoothing factor for each dataset is shown in Table 5.2.2. As is consistent with other studies (Wulder et al., 2000; Pouliot et al., 2002) changing the scale by smoothing the data decreased commission error and increased omission error. For all datasets, as smoothing increased, accuracy initially increased quickly, but slowed as it approached the optimum and then decreased at a slower rate. This trend results from the initial removal of noise by the smoothing process, which can be high and causes a substantial amount of commission error. Commission error is further decreased from the reduction of within crown variability caused by crown branches. In each dataset, commission error due to green background vegetation was not significant in relation to the above sources, however, depending on image acquisition timing and the types of vegetation present it can significantly increase commission error. At each successively greater smoothing level omission error is increased due to small crowns merging with their neighbours or flatting of the crown response. At the smoothing levels highlighted in Table 5.2.2, the two errors reach an optimal balance. Although the results were compiled for discrete smoothing intervals, they show the approximate optimal smoothing factor to be 3,2 , and $2 \sigma$ for the ESS, YCO, and OCO datasets, respectively, with associated $A I$ of $87.3,66.4$, and $71.8 \%$. The lower accuracies found for the YCO and OCO datasets are due to high omission error, likely caused by some trees being overtopped by others and hidden from the sensor, small trees with a weak response in the imagery, and multiple trees in close proximity to one another detected as a single tree. 
Table 5.2.2: Empirically derived tree detection accuracy for different Gaussian smoothing factors, $\sigma$. The optimal factors are highlighted in bold. The Correct, Omission, Commission and Total columns show numbers of trees.

\begin{tabular}{|lccccccc|}
\hline Data & Scale $(\boldsymbol{\sigma})$ & Correct & Omission & Commission & Total & \%Cor & AI\% \\
\hline ESS & 1.0 & 190 & 7 & 328 & 197 & 96.4 & -70.1 \\
ESS & 1.5 & 190 & 7 & 148 & 197 & 96.4 & 21.3 \\
ESS & 2.0 & 190 & 7 & 69 & 197 & 96.4 & 61.4 \\
ESS & 2.5 & 190 & 7 & 29 & 197 & 96.4 & 81.7 \\
ESS & $\mathbf{3 . 0}$ & $\mathbf{1 9 0}$ & 7 & $\mathbf{1 8}$ & 197 & $\mathbf{9 6 . 4}$ & $\mathbf{8 7 . 3}$ \\
ESS & 3.5 & 190 & 7 & 18 & 197 & 96.4 & 87.3 \\
\hline YCO & 1.0 & 222 & 43 & 87 & 265 & 83.8 & 50.9 \\
YCO & 1.5 & 210 & 55 & 36 & 265 & 79.2 & 65.7 \\
YCO & $\mathbf{2 . 0}$ & 201 & $\mathbf{6 4}$ & $\mathbf{2 5}$ & 265 & $\mathbf{7 5 . 8}$ & $\mathbf{6 6 . 4}$ \\
YCO & 2.5 & 194 & 71 & 21 & 265 & 73.2 & 65.3 \\
YCO & 3.0 & 190 & 75 & 19 & 265 & 71.7 & 64.5 \\
YCO & 3.5 & 187 & 78 & 19 & 265 & 70.6 & 63.4 \\
\hline OCO & 1.0 & 118 & 24 & 34 & 144 & 83.1 & 59.2 \\
OCO & 1.5 & 115 & 27 & 20 & 144 & 81.0 & 66.9 \\
OCO & $\mathbf{2 . 0}$ & $\mathbf{1 1 2}$ & $\mathbf{3 0}$ & $\mathbf{1 0}$ & 144 & $\mathbf{7 8 . 9}$ & $\mathbf{7 1 . 8}$ \\
OCO & 2.5 & 101 & 41 & 3 & 144 & 71.1 & $\mathbf{6 9 . 0}$ \\
OCO & 3.0 & 90 & 52 & 4 & 144 & 63.4 & 60.6 \\
OCO & 3.5 & 83 & 59 & 4 & 144 & 58.5 & 55.5 \\
\hline
\end{tabular}

\subsubsection{Optimal Detection Approaches}

The results of the four optimal detection approaches are shown in Table 5.2.3 and for example plots in Figure 5.2.6 for visual evaluation. Comparing these results to the empirically derived optimum reveals that no single approach outperformed the empirical results for all datasets. However, the differences in the accuracy of the best approaches and the empirical results were small and three of the approaches outperformed the empirical results for specific datasets. The better performance illustrates the potential advantage of local scale compared to global scale analysis. 
Table 5.2.3: Detection error and accuracy for the four optimal detection approaches. The Correct, Omission, Commission and Total columns show numbers of trees.

\begin{tabular}{|llcccccc|}
\hline Method & Data & Correct & Omission & Commission & Total & Correct \% & AI \% \\
\hline LMSR & ESS & 190 & 7 & 18 & 197 & 96.4 & 87.3 \\
LMSR & YCO & 201 & 64 & 25 & 265 & 75.8 & 66.4 \\
LMSR & OCO & 115 & 27 & 16 & 144 & 81.0 & 69.7 \\
LMSR & Average & $\mathbf{1 6 9}$ & $\mathbf{3 3}$ & $\mathbf{1 9}$ & $\mathbf{2 0 2}$ & $\mathbf{8 4 . 4}$ & $\mathbf{7 4 . 7}$ \\
\hline LWS & ESS & 188 & 9 & 3 & 197 & 95.4 & 93.9 \\
LWS & YCO & 192 & 73 & 18 & 265 & 72.5 & 65.7 \\
LWS & OCO & 116 & 26 & 19 & 144 & 81.7 & 68.3 \\
LWS & Average & $\mathbf{1 6 5}$ & $\mathbf{3 6}$ & $\mathbf{1 3}$ & $\mathbf{2 0 2}$ & $\mathbf{8 3 . 2}$ & $\mathbf{7 6 . 0}$ \\
\hline LSF & ESS & 190 & $\mathbf{7}$ & 41 & 197 & 96.4 & 75.6 \\
LSF & YCO & 208 & 57 & 28 & 265 & 78.5 & 67.9 \\
LSF & OCO & 115 & 27 & 9 & 144 & 81.0 & 74.6 \\
LSF & Average & $\mathbf{1 7 1}$ & $\mathbf{3 0}$ & $\mathbf{2 6}$ & $\mathbf{2 0 2}$ & $\mathbf{8 5 . 3}$ & $\mathbf{7 2 . 7}$ \\
\hline PDM & ESS & 175 & 22 & 70 & 197 & 88.8 & 53.3 \\
PDM & YCO & 210 & 55 & 48 & 265 & 79.2 & 61.1 \\
PDM & OCO & 109 & 33 & 14 & 144 & 76.8 & $\mathbf{6 6 . 9}$ \\
PDM & Average & $\mathbf{1 6 5}$ & $\mathbf{3 7}$ & $\mathbf{4 4}$ & $\mathbf{2 0 2}$ & $\mathbf{8 1 . 6}$ & $\mathbf{6 0 . 4}$ \\
\hline
\end{tabular}




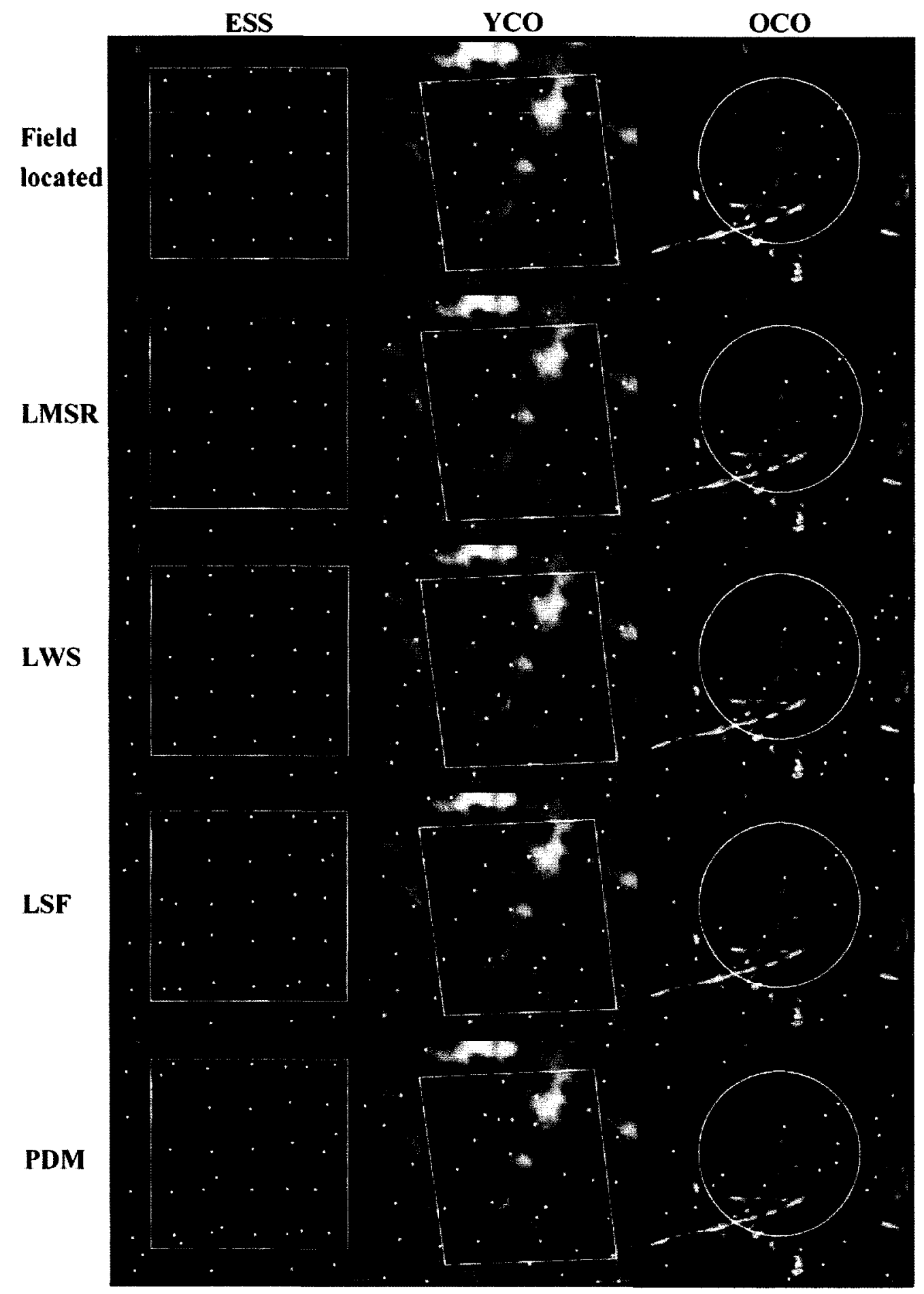

Figure 5.2.6: Example detection results for the three datasets, reference tree locations, and four detection methods compared. 


\subsection{Number of Local Maxima and Smoothing Relation (LMSR)}

The estimates of the optimal global scale using the LMSR approach are shown in Figure 5.2.7. For the ESS and YCO datasets LMSR found the same optimal smoothing level as the empirically determined optimum of 3 and $2 \sigma$, respectively. However, in the OCO dataset the optimal smoothing level was underestimated at $1.6 \sigma$. Compared to the empirical results, this difference reduced the $A I$ by only $1 \%$. Of the four approaches, LMSR was the most consistent, as it was second best in the average of each category of error and accuracy over all datasets. It is also the simplest method to implement and least parameter dependent. Only the range and interval for which the local maxima smoothing relation is desired needs to be specified. If incorrectly specified, the results will clearly reveal such errors, as the relation will be difficult, if not impossible to interpret.

Processing time may be high for large datasets, but was not slower than any of the other methods tested here. A simple solution if processing time becomes problematic is to sample the imagery for analysis.
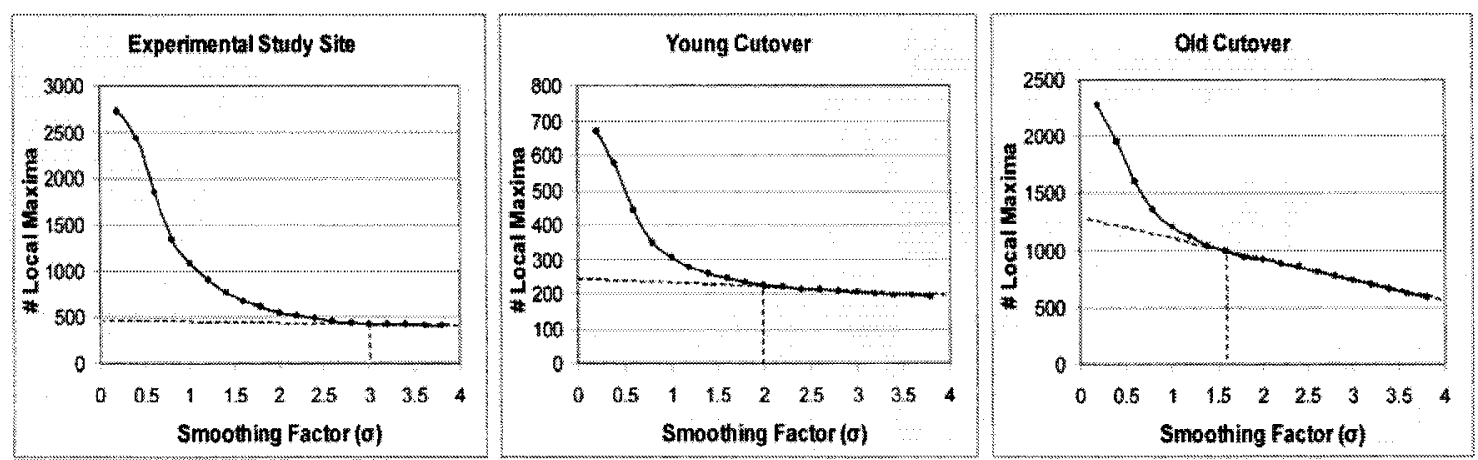

Figure 5.2.7: Local maxima smoothing relation for the three datasets used in the study. Dashed lines indicate the visual method used to determine the optimal Gaussian smoothing factor.

The main disadvantage of the LMSR approach is that it is subjective and requires users to calibrate themselves to ensure that consistent results are obtained. We had two additional 
colleagues examine the LMSR curves generated in this study and found that the estimated smoothing factor varied by $\pm 0.2 \sigma$. For the data presented here, this would produce changes in $A I$ of approximately $4 \%$. This approach is also limited by the degree to which commission and omission error can be minimized using a single global scale. For example, consider a dataset with two quite different dominant crown sizes with each crown size being equally represented. The larger crowns may not be detected as single crown objects without applying such a high level of smoothing that many of the small crowns are omitted. Fortunately, such extreme conditions appear to be rare, but users should be aware that the method only seeks to achieve the optimum balance of omission and commission error. In cases where the crown size distribution is skewed or bimodal as in the example above, this approach will find the optimal error balance, but less frequent crown sizes will have greater detection error. Clearly, the best results will be obtained when the crowns sizes are equally distributed with a narrow standard deviation.

\subsection{Variable Sized Local Sample Windows (LWS)}

The LWS approach produced the highest average accuracy index of all methods, but was not the most consistent. It produced very high accuracy for the ESS dataset, which was a highly controlled experiment with the least variable crown sizes and constant tree spacing. These are ideal conditions for this approach because it minimized error caused by large sample windows containing several trees. In the two operational regeneration datasets with inconsistent spacing and more variable crown sizes this method performed relatively poorly. It also required specification of the local transect sample distance, the 
number of transects, and the $\mathrm{r}^{2}$ scaling factor. Of these, the most critical is the sample distance specification, as too large a sample distance may extend over more than one crown even after transect scaling. This can occur when a small crown is next to a larger one, in which case the polynomial achieves a sufficient fit with the larger crown because the small crown is treated as noise. Thus, this approach appears to be ideally suited for even-aged plantation forests with equally sized and spaced crowns, as a constant sample distance can be specified that is appropriate to all crowns in the imagery. Further, the constant tree spacing greatly reduces the problem of large window sizes containing more than one tree crown.

\subsection{Local Smoothing Factor (LSF)}

The LSF approach performed the best of all methods with the datasets that had highly variable crown sizes and tree spacing (YCO and OCO). For the OCO dataset, which had the highest crown size variability, the observed $A I$ was $\sim 5 \%$ higher than the next best optimal detection approach. This is an important characteristic because these datasets are representative of more operational conditions. Further, it shows that local approaches are able to achieve higher accuracy than global approaches. However, the improvement comes at the cost of an increased number of parameters and parameter sensitivity. Such parameter dependence strongly limits the utility of an approach as it becomes more difficult to understand the nature and sources of errors. The poor results obtained for the ESS dataset suggest that the heuristic equation defined to convert the crown size estimates to smoothing factors was inappropriate for this dataset. To better evaluate the effect of the equation on the results, the slope of the original equation was increased to 
0.35. Using this slope an $A I$ of $80.3 \%$ was obtained, representing an increase of about 5 $\%$. This clearly illustrates that this approach is very sensitive to the user-defined equation and it is unlikely that it would be useful for operational applications. Further research may identify improved methods to determine this equation in a more consistent and objective manner than that used here. Although this approach had several limitations, an approach designed to achieve the same objectives, i.e. local scale optimization, is needed to provide the highest possible accuracy using automated tree detection in high-resolution imagery. Currently, no method has been identified that can accomplish this task.

\subsection{Post Detection Merging (PDM)}

The PDM approach produced the lowest accuracies of all methods tested. This is due in part to the global threshold used for the merging decision, which is unable to account for local variations in brightness that produce variations in the depth of brightness valleys between crowns in the imagery. Further, using the maximum value of the common boundary between two crowns often led to incorrect merging decisions. This occurred when a small portion of the shared boundary contained brightness values similar to the local maxima of the crown segments being compared, but the majority of the boundary distinctly divided the two segments and suggested that they should not be merged. Using the average brightness value of the common boundary produced poorer results because the average was biased by the darker edges of the crown making the boundary appear stronger than it actually was and leading to numerous merges being missed. Due to these limitations, this approach is not useful as an error minimization approach on its own, but 
could be useful as a low priority check to merge trees that strongly meet defined merge criteria.

\subsubsection{Comparison with Other Research}

It is difficult to compare the results of this research with other studies because most were conducted in mature forests, and sensor types and initial image resolutions were not the same as that used here. With regard to sensor and image characteristics, two of the most critical are spatial resolution and sun-sensor geometry. The spatial resolution dictates the number of omission errors as some crowns may be too small to be detected at larger resolutions and other crowns that are closely spaced may be merged into a single crown. The methods presented in this study and reviewed below require very high spatial resolution imagery in relation to the crown size. However, if large area coverage is required, coarser resolution must usually be used with an associated reduction in individual tree detection accuracy. For example, Gougeon and Leckie (1999) have taken this approach to emulate more operational conditions for automated detection in regenerating forests. Their results were promising under the design criteria, but as expected, numerous omission errors were observed. The sun-sensor geometry is another important factor as it affects both the radiometric and geometric properties of the tree crowns. Using simulated imagery of mature eucalyptus forests, Culvenor (2000) showed that tree detection accuracy was best with small off nadir view angles, with back scattered as opposed to forward scattered radiance, and at higher solar zenith angles. 
Forest properties control how visible and distinct crowns are in images. Coniferous trees are generally much easier to accurately detect than deciduous species due their distinct radiometric crown morphology (Warner et al., 1999). The results presented in this study showed the effects of varying tree size, density, and spatial pattern with different detection approaches identified as optimal depending on these conditions. A distinct difference between mature and regenerating coniferous forests is that mature forests typically have reduced occurrence of trees in close proximity due to self thinning caused by inter and intra-specific competition. The greater spacing between trees will generally enhance the distinct nature of individual crowns, improving detection results. However, mature forests tend to have greater vertical structure than regenerating conditions and trees in the lower canopy positions can be difficult to detect if hidden from the sensor or strongly shaded by other crowns. This can also occur in regenerating forests, where smaller trees are hidden by larger ones, but it is less frequent.

Variations in accuracy evaluations also render comparisons amongst studies difficult. In regeneration conditions, it is a simpler task to create field maps of tree locations. In mature forests greater error in locating individual trees is likely due to errors associated with classical or GPS survey techniques. Accuracy evaluations vary with regard to the types of comparisons made, including plot based vs. single tree based, manually interpreted vs. field data, and the selection of data to use as reference. For example, Gougeon (1995a) compared automated results to ground counts on a plot basis and on an individual tree basis with manual interpretation. Erikson (2003) did not include commission error occurring in areas considered as non-crown background. Validation by 
manual interpretation also tends to produce higher accuracy measures than field based validation because it does not include errors occurring from trees not visible to the sensor or from two trees in close proximity that appear as a single crown. For example, $A I$ calculated from Brandtberg and Walter's (1998) results was $8 \%$ less using field reference data than using reference data from image interpretation.

Despite a lack of capability for rigorous comparison amongst studies, it is still of interest to review previous research results and theoretical aspects in light of this study. In the following, where possible, the accuracy values published in other studies have been converted to the $A I$ (Equation 5.2.2) used in this study for comparison purposes. One approach that was not considered for this study is scale space analysis. It has been well developed and is theoretically well justified in computer vision research as a means of extracting basic image structures over multiple scales. Thus, it appears to be well suited for automated tree detection. However, current scale space approaches have not shown significantly improved accuracies over other methods and are often dependent on the definition of many hard to define parameters. The scale space edge detection method proposed by Brandtberg and Walter (1998) showed detection $A I$ to be $59 \%$ in mixed mature European forests. The method required several input parameters $\backslash$ thresholds and it was noted that it had difficulty detecting crowns that did not have rounded or circular shapes. In another study using lidar data of leaf-off mature forests in the eastern United States, Brandtberg et al. (2003) determined suitable parameters for a scale space approach based on blob detection using field data. Other more simple approaches have produced reasonable results and are equally or less parameter dependent. The variable window 
sized approach tested in mature natural and plantation forests by Wulder et al. (2000) produced an $A I$ of $51 \%$. In regeneration conditions, Pouliot et al. (2002) using the same approach with the ESS dataset used in this study found an $A I$ of $89 \%$. The slightly higher results reported in this paper are due to improvements in the extraction of crown pixels, using classification rather than simple thresholding. Although, the variable window size approach has been successful, as shown in this study it is not appropriate for forests with highly variable crown sizes and spacing. Local binarization has been tested as a means to improve results of local maxima crown detection. In mature European forests of varying species compositions, Pitkanen (2001) observed $A I$ values ranging from 48 to $96 \%$ depending on parameter values used and specific forest conditions. Improvements with local binarization ranged from 0 to $4 \%$. A local surface fitting method was used by Persson et al. (2002) to determine which of three scales was most appropriate for automated tree detection. The approach worked well with lidar data of Norway spruce forests producing an $A I$ of $70 \%$. The main disadvantage of the approach is that the scales were discrete and selected arbitrarily. Further, it is not clear what the advantages and disadvantages of lidar were compared to optical data for tree detection using this method. Leckie et al. (2003b) found the two data sources to be complementary, each having its own unique advantages. In their study, lidar data proved to be better suited for more open forests and optical data better in moderate to closed canopy conditions. Erikson (2003) used a region growing approach, where the size of the crown to be segmented was estimated using the largest curved segment from a cluster determined by image binarization. Reported accuracy for the mature European forest of mixed composition was $A I \sim 81 \%$ with manually interpreted delineations. As with many region growing 
algorithms the method requires numerous parameter specifications to achieve accurate results.

The results of this study show that the approaches tested produced accuracies within the range reported in other studies. Further, as shown in other studies the accuracy obtained was dependent on the specific forest conditions and number of parameters used. Accuracies can be high for methods that utilize numerous well-estimated parameters. Other more general methods produce lower accuracies, but are less parameter dependent making them more widely applicable to a range of forest conditions. Thus, the choice of the optimal method depends on the required accuracy, acceptable cost, and forest conditions.

\subsubsection{Conclusion}

This paper has presented a direct comparison of different optimization approaches for automated tree detection within a range of datasets representing a variety of sensor and scene characteristics of regenerating forests of varying structure. Of the approaches tested, determination of the optimal global smoothing (LMSR approach) factor produced the most consistent accuracies, was typically second highest in accuracy, and was the least parameter dependent. Its major limitation was that it requires a moderately subjective assessment of the relation between the number of local maxima and smoothing intensity, which requires user calibration to obtain consistent results. Determining an optimal local window size (LWS approach) worked extremely well with the dataset consisting of constant tree spacing and crown sizes, while determining the optimal local 
smoothing factor (LSF approach) produced the highest accuracies in the more variable tree size and spacing datasets. However, both of these approaches were dependent on appropriately defined input parameters. The post-detection merging (PDM) approach did not perform sufficiently well to merit further consideration.

Image scale is a critical component in automated tree detection, whether it is represented by the smoothing level, window size or another form of size parameter. Using a global scale representation such as a constant window size, smoothing factor, or threshold can only lead to a certain level of tree detection accuracy. Local methods have the potential to achieve theoretically perfect accuracy, but have proven technically difficult to implement. The two local approaches tested here were encouraging, but their reliance on parameters is considered too limiting for more operational applications. Further research will focus on identifying additional approaches for optimal detection as well as combining some of the more promising ones into an integrated approach.

\subsubsection{Acknowledgements}

This research was funded by a grant to D. King and scholarship to D. Pouliot from the Natural Sciences and Engineering Research Council of Canada (NSERC). Image data for the ESS and OCO sites were collected by the Ontario Ministry of Natural Resources Data Acquisitions Branch. Field data for these sites were collected by the Ontario Ministry of Natural Resources. Image data for the YCO were acquired by Wiskair Ltd. Duncantech Inc. (now Redlake Inc.) generously loaned the MS3100 digital camera. Field data collection for the YCO dataset were greatly aided by the assistance Mark Lindsay. 
Buchanan Forest Products Ltd. provided historical site information and logistic support for image and field data collection of the YCO dataset. Thanks to Wayne Bell of the Ontario Forest Research Institute and Doug Pitt of the Canadian Forest Service for advice and support in this research. 


\title{
5.3 DEVELOPMENT AND EVALUATION OF AN AUTOMATED TREE DETECTION-DELINEATION ALGORITHM FOR OPERATIONAL MONITORING OF REGENERATING CONIFEROUS FORESTS
}

Citation: Pouliot, D.A., D.J. King, and D.G. Pitt (2005). Development and evaluation of an automated tree detection-delineation algorithm for monitoring regeneration coniferous forests. Canadian Journal of Forest Research, 35: 2332-2345.

\begin{abstract}
An algorithm is presented for automated detection-delineation of coniferous tree regeneration that combines strategies of several existing algorithms, including image processing to isolate conifer crowns, optimal image scale determination, initial crown detection, and crown boundary segmentation and refinement. The algorithm is evaluated using $6 \mathrm{~cm}$ pixel airborne imagery in operational regeneration conditions typically encountered in the boreal forest 5-10 years after harvest. Detection omission and commission errors, as well as an accuracy index $(A I)$ combining both error types were assessed on a tree-by-tree basis, on an aggregated basis for each study area, in relation to tree size, and in relation to the amount of woody competition present. Delineation error was assessed in a similar manner using field measured crown diameters as reference. Individual tree detection $A I$ improved with increasing tree size and was $>70 \%$ for trees larger than $30 \mathrm{~cm}$ crown diameter. Crown diameter absolute error measured from automated delineations was $<23 \%$. Large crown diameters tended to be slightly
\end{abstract}


underestimated. The presence of overtopping woody competition had a negligible effect on detection accuracy and only reduced estimates of crown diameter slightly.

\subsubsection{Introduction}

Sustainable forest management depends on successful regeneration of disturbed forest areas. To ensure regeneration success, forestry professionals require timely information regarding the abundance, distribution, and size of crop trees and non-crop (e.g. nonconiferous vegetation) competitors. Currently, field based monitoring is the principal means used to collect information regarding regeneration status. Such monitoring is usually conducted by qualitative visual assessment andlor quantitative assessment using statistically based plot sampling designs (Pitt et al., 1997). The objective of the former is to utilize expert knowledge in rapid collection of a large amount of information, but the procedure is highly subjective. Plot based assessment methods minimize subjectivity, but are highly labour intensive and costly.

Remote sensing based methods have the potential to provide the required information in a more objective manner, at lower cost, and with greater coverage than is attainable using field sampling. The application of remote sensing for regeneration assessment has, to date, largely involved manual interpretation of large-scale photography (LSP). Results have shown that useful estimates of conifer stocking, species, crown size, health condition, and stratification of key vegetation complexes can be made (Goba et al., 1982; Hall, 1984; Hall and Aldred, 1992; Pitt and Glover, 1993; Pitt et al., 2000). However, these methods have not found widespread use because they tend to be highly specialized, 
time consuming, and subjective, requiring specially trained personnel and equipment (King, 2000).

Automated tree detection-delineation using high spatial resolution remotely sensed imagery provides a potentially efficient means to acquire information needed for forest regeneration management decisions. Tree detection can provide estimates of tree abundance and spatial pattern useful for evaluating density and stocking objectives, as well as inputs for growth modeling. Delineation of individual tree crowns can potentially enhance species composition inventory through analysis of within-crown spectral data (Leckie et. al., 2003a; Leckie et al., 2005), spatial data (Haddow et al., 2000; Erikson, 2004), lidar data (Holmgren and Persson, 2003), and crown shape (Brandtberg, 1999). Recent research has also shown the potential for damage mapping due to insects and disease (Leckie et al., 2004). Further, delineated crown dimensions can be used to model tree structural variables such as height, volume, or biomass. (Culvenor, 2000; Hayward and Slaymaker, 2001; Persson et al., 2002).

Much of the research on image-based automated tree detection-delineation has focused on mature forests for purposes of forest inventory. Techniques are varied, but generally utilize the same basic radiometric properties of tree crowns. These key properties are the association of the approximate crown apex with a local maximum image brightness value and decreasing brightness towards the crown edges. This radiometric crown model has been described as analogous to that of a mountainous landscape, where peaks are the highest elevation approximately representing crown apexes and surrounding valleys are 
the lower elevations representing the space between crowns or, where crowns overlap or touch. Some algorithms are explicitly linked to this concept. One of the first, developed by Gougeon (1995a), incorporates a procedure to outline the local brightness 'valleys' between crowns. A rule set is then applied to the valley network to refine crown boundaries and split and merge crowns. However, the majority of algorithms approach detection-delineation as two separate tasks. These methods typically apply some method of local maximum detection to first detect crowns, and then use these as reference points for crown delineation. Methods applied for tree detection include: 1. enhancement and thresholding (Dralle and Rudemo, 1996; Walsworth and King, 1999) where a global image operation such as smoothing or high-pass filtering is applied and the resulting pixel brightness values within a defined range are extracted as estimates of tree locations, 2. template matching (Pollock, 1999) involving the correlation between a geometricradiometric tree crown model and image data, 3. multiscale edge detection (Brandtberg and Walter, 1998) where the occurrence of edges over several image scales is examined to define a region in which the brightest pixel value is taken as a representative location of the tree apex, 4. local maximum filtering (Pinz, 1999; Culvenor, 2000; Gougeon and Leckie, 1999; Niemann et al., 1999; Wulder et al., 2000; Pitkanen, 2001; Pouliot et al., 2002; Erikson, 2003) where the maximum pixel brightness value in a moving window sample of a specified size is taken to represent the tree apex, and 5. gradient following (Persson et al., 2002) where maxima are identified by following the local upward gradient from a given pixel to the brightest local value. 
Tree delineation has been accomplished mostly through forms of: 1. spatial clustering (Brandtberg and Walter, 1998; Walsworth and King, 1999; Erikson, 2003) involving the identification of groups of pixels related by defined criteria, 2. valley detection based on local minima (Gougeon, 1995a; Persson et al., 2002), 3. local edge detection (Pouliot et al., 2002), and 4. a combination of valley detection and spatial clustering (Culvenor, 2000). A more detailed review of detection-delineation algorithms is provided in Culvenor (2003).

Each of these algorithms has been shown to provide reasonable results in the specific imaging and scene conditions used. However, the complementary benefits of these approaches have not been combined into an integrated algorithm that may be robust under a variety of operational forest conditions. In addition, very little development and evaluation has been carried out in regenerating forests where canopies tend to range from open to closed over relatively fine spatial scales and crown sizes can be highly variable. In regenerating forests, algorithms developed by Gougeon and Leckie (1999) and Pouliot et al. (2002) have been evaluated. The results of these studies were encouraging, but both were conducted in controlled experimental study sites that were not representative of typical post-disturbance conditions that would exist after clearcut harvesting, planting with natural ingress, or intense fire. In these conditions, tree size and spacing is considerably more variable, competing woody stems often overtop conifers, topography is wide-ranging, and ground brightness is more inconsistent. Thus, it is difficult to determine if either of the approaches would provide suitable results in more operational conditions. 
This research was designed to address the issues identified above, i.e. to combine beneficial and complementary aspects of existing detection-delineation algorithms to produce a new algorithm that is robust under the diverse conditions encountered in typical conifer regeneration conditions. Specifically, the objectives of the research were:

1. Develop an integrated and robust tree detection-delineation algorithm for operational regenerating coniferous forest conditions imaged with high spatial resolution imagery.

2. Evaluate the algorithm accuracy and sources of error in relation to tree size and amount of woody competition.

\subsubsection{Methods}

\subsubsection{Study Site}

The study site consisted of three operational cutovers located northeast of Sioux Lookout, Ontario, in the Buchanan Inc. forest management unit. The three cutovers represent various regeneration conditions, most distinctly marked by differences in competing vegetation levels that reflect different soil and microclimate conditions. These sites were labeled High $\left(50^{\circ} 46^{\prime} \mathrm{N}, 91^{\circ} 25^{\prime} \mathrm{W}\right)$, Moderate $\left(50^{\circ} 18^{\prime} \mathrm{N}, 91^{\circ} 39^{\prime} \mathrm{W}\right)$, and Low $\left(50^{\circ} 50^{\prime} \mathrm{N}\right.$, $91^{\circ} 21^{\prime} \mathrm{W}$ ) competition (Figure 5.3.1). 


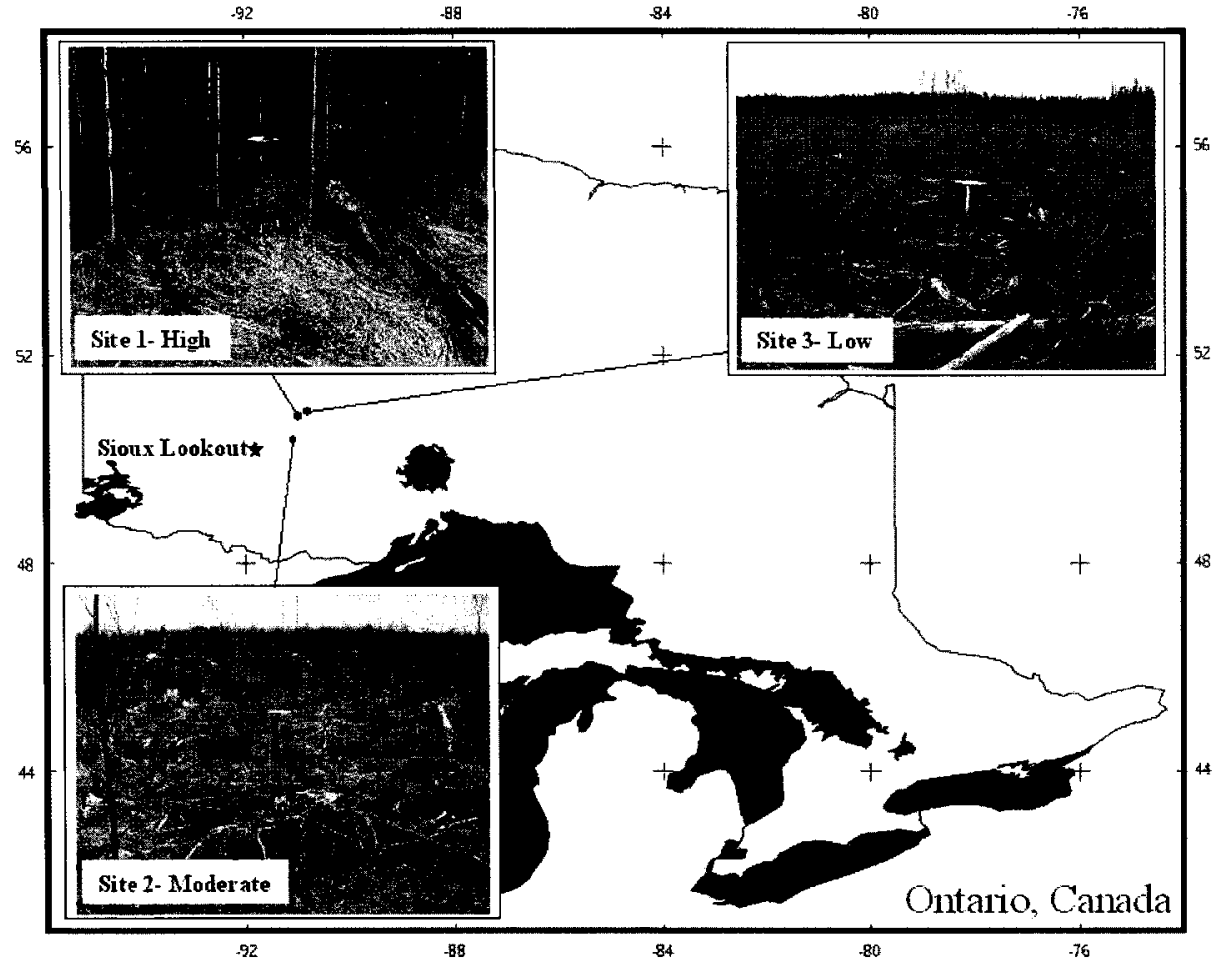

Figure 5.3.1: Study site locations.

The High site was planted in 1992 with white spruce (Picea glauca). Species composition at the time of this study was $89 \%$ white spruce, $3 \%$ jack pine (Pinus banksiana), and $8 \%$ balsam fir (Abies balsamea). Total conifer density was 1784 stems $\mathrm{ha}^{-1}$. The average crown diameter was $77 \mathrm{~cm}($ standard deviation $(\mathrm{sd})=44 \mathrm{~cm})$ ranging from 12 to $290 \mathrm{~cm}$. The large crowns in the site were advanced regeneration that was present post disturbance. The average tree height was $138 \mathrm{~cm}$. The site supported a variety of herbaceous and woody non-crop vegetation species, indicative of rich soil with moderate moisture. Woody vegetation was abundant with an approximate density of 8500 stems ha ${ }^{-1}$ for trees above $2 \mathrm{~m}$ in height. This layer was dominated by alder (Alnus spp.), but also contained aspen (Populus tremuloides). Both species considerably overtopped 
the crop trees. Grass was the most abundant herbaceous vegetation, occurring in patches where woody competition density was low. Topography was slightly sloping towards the southwest, with occasional small drainage channels. The size of the cut area was 11 ha of which the image transect sampled approximately $20 \%$.

The Moderate site was planted with black spruce (Picea mariana) in 1997, but in 2002 species composition was about $50 \%$ jack pine due to natural ingress. Total conifer density was $3172 \mathrm{stems} \mathrm{ha}^{-1}$. The average crown diameter was $59.5 \mathrm{~cm}(\mathrm{sd}=33 \mathrm{~cm})$ ranging from 6 to $135 \mathrm{~cm}$. Average tree height was $110 \mathrm{~cm}$. Competition consisted of pockets of high-density aspen and lower density white birch (Betula papyrifera) with an overall density of 3400 stems $^{-1} a^{-1}$ for trees above $2 \mathrm{~m}$ in height. Aspen tended to be considerably taller than birch, and both typically overtopped the spruce and pine trees. Herbaceous vegetation was dominated by grass and moss in moist valley areas. In higher areas, low woody shrubs were abundant. Topography was rolling, with associated wet areas in depressions and dry areas on higher ground. The size of the cut area was 20 ha of which the image transect sampled approximately $18 \%$.

The Low site consisted of a mixture of jack pine and black spruce, with a species composition of 30:70, respectively. Spruce was planted in 1995 and was typically smaller than the natural ingress pine. Total conifer density was $4670 \mathrm{stems} \mathrm{ha}^{-1}$. The average crown diameter was $55 \mathrm{~cm}(\mathrm{sd}=39 \mathrm{~cm})$ ranging from 5 to $210 \mathrm{~cm}$. The average tree height was $93 \mathrm{~cm}$. The site had little to no competing vegetation with no woody competitors greater than $2 \mathrm{~m}$ in height. Woody species included alder and willow (Salix 
spp.), while herbaceous competitors included Labrador tea (Ledum groenlandicum), mosses, lichens, and some sparse grasses. Topography was similar to the Moderate site, with slightly rolling hills and associated wet and dry areas. The size of the cut area was 7 ha of which the image transect sampled approximately $34 \%$.

\subsubsection{Field Data}

Sample plots were selected along a predetermined transect, with the objective of sampling the full range of conifer tree species, tree density, crown sizes, and woody competitor abundance on each of the sites. Circular plots were used with a radius of $3 \mathrm{~m}$. For visual reference in subsequent imagery, plot centres were marked with a $40 \times 40 \mathrm{~cm}$ white board, mounted on a $1.0 \mathrm{~m}$ tall stake. Each tree within the plot was assessed in relation to the plot centre by measuring the distance and direction from the centre to the tree. For each conifer tree in the plot, the species, stem height, stem diameter at ground level and crown diameter (oriented north-south and east-west) were recorded. Position and tree measurement data were used to create a GIS layer for validation of automated detection-delineation results. The height of each woody stem within the plot was also recorded as a measure of competition.

\subsubsection{Image Data}

Images were acquired under leaf-off conditions on May 11, 2002 between 11:30 - 15:00 local time using a Duncantech MS3100 CIR digital camera with a $14 \mathrm{~mm}$ focal length Sigma aspherical lens. The camera was mounted in the front bay of a boom system 
attached to the undercarriage of a Bell Ranger helicopter. The camera uses beam splitting optics to separate irradiance into three spectral bands that are imaged simultaneously by three charged coupled devices (CCD). The spectral bandwidths were: green (500-600 $\mathrm{nm})$, red (600-700 nm), and near-infrared (NIR, 700-900 nm). Each CCD was comprised of $1300 \times 1000$ photosites, which translated to 8 -bit multispectral images of the same format. Shutter speed was set individually for each band to $1 / 200 \mathrm{~s}, 1 / 143 \mathrm{~s}$, and $1 / 111 \mathrm{~s}$, respectively by viewing histograms of the target areas in flight. A flying height of $182 \mathrm{~m}$ was used to capture images with $\sim 6 \mathrm{~cm}$ nominal ground pixel size and a spatial coverage of $83 \times 60 \mathrm{~m}$. Flying speed varied between 2.2 and $3.3 \mathrm{~m} \mathrm{~s}^{-1}$.

\subsubsection{Tree Detection-Delineation Algorithm}

The detection-delineation algorithm developed for this study consists of the four stages shown in Figure 5.3.2. The following sections describe in detail the specific processing carried out in each stage.

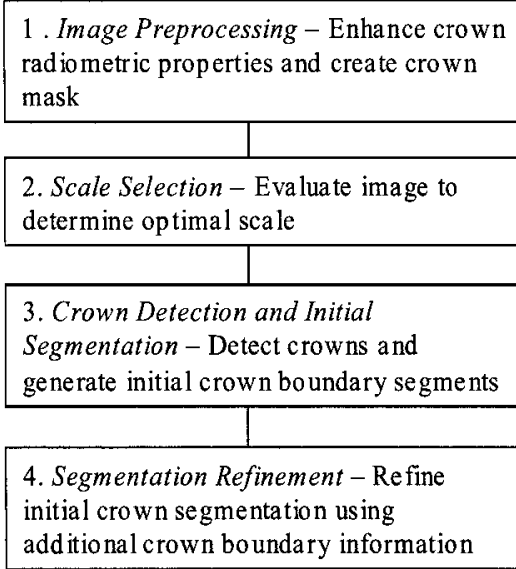

Figure 5.3.2: Flow chart showing stages involved in algorithm implementation. 


\subsection{Image Preprocessing}

Image preprocessing is a flexible stage where the user selects the best spectral band andlor enhances the image data to maximize crown distinction from background cover types and thus optimize algorithm performance. Common enhancements can include filtering to remove image noise and image transforms such as band ratios, or the intensity or hue image from an IHS colour space transformation. The choice of enhancements depends on the imagery and requires some experimentation. For example, in Pouliot et al. (2002), a smoothed hue image was found to be the best enhancement for Kodak DCS 460 colour IR imagery. In the present study, visual examination of the imagery revealed that the crown apexes and boundaries were most distinct in the NIR band and that image transformations and smoothing were not required.

Following initial band selection or enhancements, a conifer vegetation mask must be created to remove as much other vegetation (typically low-lying ground vegetation such as mosses) that is also green at this time of year. It can be created in a number of ways, but typically simple thresholding is sufficient or classification methods may be used if multispectral data are available. The conifer mask is used in all subsequent processing stages.

For this study, the original three spectral bands were classified into 100 clusters using the ISODATA algorithm (Jensen, 1996) and aggregated into two final classes, conifers and non-conifers. This hyper-clustering approach was used because it allows for full control of the merging process and does not require the user to explicitly define class spectral 
properties, as is the case with supervised approaches. The binary mask created from the clustering process was dilated using a $3 \times 3$ structuring element to remove small holes in the mask and to ensure that the full extents of crowns were covered. The NIR image data under the mask were then extracted for the detection-delineation processing.

\subsection{Scale Selection}

The scale at which imagery is acquired is often not the optimal scale for automated tree detection. At fine scales, small trees are more likely to be detected, but within-crown branching of large crowns can cause numerous false detection errors due to local maxima associated with within crown branch clusters. At coarser scales, small trees may be missed or overlapping trees may be detected as a single crown. Thus, a scale optimization step is required to identify the scale that maximizes the detection of small trees while minimizing the detection of large crown branches and other image artifacts as individual crowns. Pouliot and King (2005a) evaluated four approaches for optimal conifer detection in three different regenerating forest conditions using aerial photography and digital camera imagery. The best, and that used in this study, was adapted from a global approach presented in Culvenor (2000). In this approach the relation between scale simulated using a Gaussian filter and the number of local maxima identified (i.e. local maxima smoothing relation (LMSR)) was used to define the optimum scale. In examining this relation (Figure 5.3.3), initial smoothing levels remove image noise and the number of local maxima drops rapidly with scale. This decrease then beings to slow down as noise is further removed and within-crown branching starts to become the major source of additional local maxima. Further smoothing removes local 
maxima caused by within crown branching, but also starts to remove small crowns, leading to omission error. Hence, the need to determine the point that optimally balances the two error sources. An automated approach to selection of the optimal scale from the LMSR was suggested by Culvenor (2000) based on the maximum rate of change in the second derivative, but tests of this approach found it to be highly sensitive to local variations in the LMSR. Attempts to improve the automated selection procedure by applying smoothing algorithms (local averaging, Gaussian smoothing, and Lowess smoothing) to remove these variations revealed a strong dependence on the parameters selected. No logical criteria for parameter selection could be identified, making the selection an arbitrary and trial and error process. Consequently, a more robust visual assessment procedure was adopted as described below.

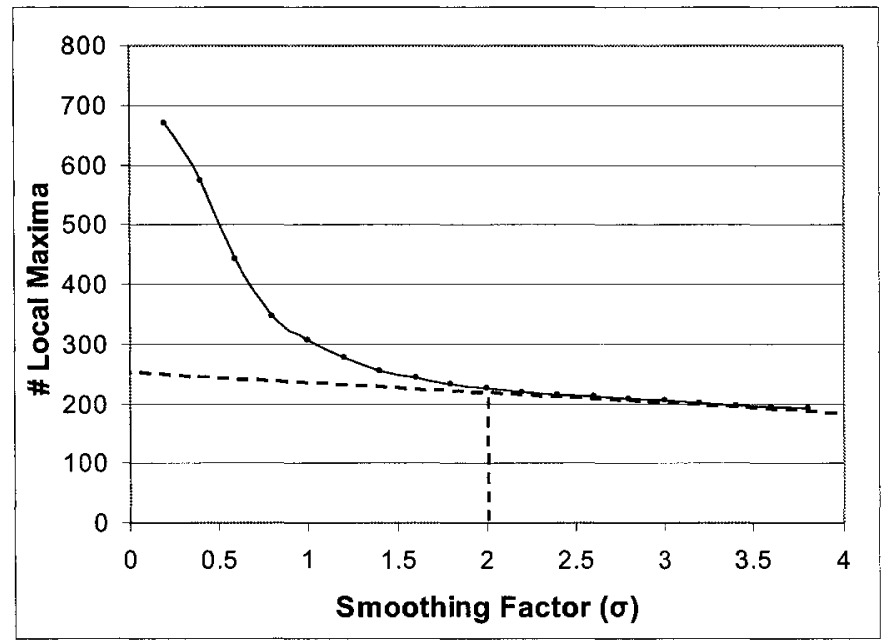

Figure 5.3.3: Solid line - example local maxima smoothing relation. Dashed line - indicates the visual rule used to determine the optimal Gaussian smoothing factor.

The first step in visual assessment involved plotting the LMSR (Figure 5.3.3). Then, the longest line that could be fit to the LMSR curve was determined, starting with the last point and moving towards the first point. This was defined as a linear fit where residuals 
were, in relative terms, small and equally distributed on either side of the line. In the final step, the point where this line and the curve started to diverge was determined. This point was taken as an indication of the optimal level of smoothing required. In this implementation, the LMSR was determined using a step size of $0.1 \sigma$ within a range of 0 5. This scale selection approach is advantageous in that it does not require field data and provides information on the scaling properties of the crowns in the imagery that can be used to estimate a scale close to that of the optimum. Although repeatable (in tests of independent users), the main disadvantage is that it is subjective and requires users to calibrate amongst themselves. It is also limited by the degree to which commission and omission error can be simultaneously minimized using a single global scale.

\subsection{Crown Detection and Initial Segmentation}

Crown detection and initial segmentation were carried out using a watershed algorithm approach adapted from Persson et al. (2002), who applied it to lidar data in mature forest conditions. In this method, each pixel in the image is considered a seed and forced to follow the local upward gradient until a local maximum point is reached. This seed pixel is then assigned to the clusterlsegment for that local maximum position. The results of the algorithm produce an image with the same positions of local maxima as those obtained using a $3 \times 3$ local maximum filter. However, it also provides an initial segmentation of the crowns based on the cluster defined by pixels that climbed to a given local maximum point. 


\subsection{Segmentation Refinement}

The initial segmentation results from the gradient-following procedure are highly dependent on the quality of the crown mask created in Stage 1, which, in turn, is dependent on the quality of the image data used and image processing performed to create the mask. For example, brightness variations due to bi-directional and optical light fall-off effects can lead to the removal of crown pixels or addition of non-crown pixels, depending on the criteria used to separate crown and non-crown pixels. The purpose of the refinement step is to minimize this dependence by locally assessing the boundaries for each crown based on the initial gradient following results. The refinement step was adapted from Pouliot et al. (2002). Starting at each local maximum, a user-specified number of transects around the candidate crown object are extended from the local maximum out to the local minimum boundary defined by the initial segmentation results, plus one additional pixel. The transect data are then extracted and the maximum value in the first derivative is taken as the most suitable crown boundary position. In this study, crown boundaries were detected using the original unsmoothed imagery to avoid boundary distortion due to smoothing.

\subsubsection{Detection-Delineation Evaluation}

In mature forests, difficulties and costs associated with locating and measuring trees in the field have led to a large diversity of accuracy assessment procedures. However, in regenerating conditions it is easier to locate and measure trees in the field due to the reduced vertical structure. Thus, for validation, field based measurement was conducted. Here, we present a standardized accuracy assessment procedure that can be used with 
field measurements to provide summary and in-depth information regarding detectiondelineation errors.

\subsection{Visual Assessment}

One of the most effective means to acquire insight regarding processing results is visual evaluation. A set of four plots was selected prior to algorithm implementation that represented the unique characteristics of each transect. These plots included small trees, clustered groups of trees, and plots with high levels of woody stem competition. The detection-delineation results for these plots were examined to assess the general accuracy, identify errors, error commonalties, and error sources.

\subsection{Quantitative Assessment of Detection Results}

Detection accuracy was quantitatively evaluated using a method adapted from Pitkanen (2001), where points from automated tree detection are assigned to ground measured reference locations based on an iterative search distance algorithm. In the first iteration, all detected tree points from the automated processing within a given search distance of a reference tree location are found, the overlap between the segments for these points calculated, and the largest overlap assigned as a match to the reference point. Following this, the matched automated point and the reference point are removed from further consideration so a given reference tree cannot be assigned to more than one detected tree. Overlap is taken as the average from both perspectives, i.e. image to field segment and field to image segment. A minimum overlap threshold is also used to ensure that small 
overlapping segments are not considered. In subsequent iterations, a larger search distance is used up to a maximum search distance of approximately half the size of the average tree crown size in the dataset. In this study, the initial search distance was $0.1 \mathrm{~m}$ and the maximum search distance was $0.3 \mathrm{~m}$, with the search distance increasing by 0.1 $\mathrm{m}$ for each iteration. The minimum overlap threshold was set at $20 \%$. After the algorithm is complete, the reference trees not matched are taken as omission error, the detected trees not matched to a reference tree are taken as commission error, and the matched segments are considered correctly identified trees. The sizes of the test and reference segments associated with each match were also recorded so that the relation between detection accuracy and tree size could be evaluated. Detection results for minimum tree sizes of 30 and $60 \mathrm{~cm}$ were evaluated. The numbers of omission, commission, and correctly identified trees are reported along with an accuracy index (Pouliot et al., 2002) calculated as:

$$
A I=[(n-o-c) / n] \times 100
$$

where $n$ is the actual number of trees in the study area; and $o$ and $c$ are the numbers of omission and commission errors respectively. With this index, both omission and commission errors are incorporated into a single summary value. Negative $A I$ values are possible and occur when commission and omission errors are greater than the total number of trees counted in the field. 
As the results of the detection algorithm were specifically for the image-based global smoothing factor selected using the procedure described previously, it was of interest to know what the accuracy would be if the optimal global smoothing factor was determined empirically through use of iterative accuracy assessment. To do this, each transect was smoothed using a Gaussian filter, with smoothing factors ranging from 1 to 3.5 in increments of $0.5 \sigma$. Local maxima were detected using the gradient following method, and accuracy results were compiled using the field data as described above. The optimal factor was taken as that which produced the highest accuracy index.

\subsection{Quantitative Assessment of Delineation Results}

Delineation accuracy was assessed by comparing the average crown dimensions for the matched trees using root mean square error (RMSE) and relative RMSE\% calculated as:

$$
R M S E \%=\frac{\sqrt{\frac{1}{n} \sum\left(P_{i}-O_{i}\right)^{2}}}{\bar{O}} \times 100
$$

where $n$ is the number of observations, $P_{i}$ is the predicted value from automated delineation, $O_{i}$ is the observed value in the field, and $\bar{O}$ is the mean of the observed values. Mean absolute error (MAE) was also reported, as it is more robust to outliers, which can dramatically inflate RMSE values. Delineation error was evaluated first for all matched trees and second for those matched trees where only one automated segment was found to overlap a field reference ellipse by greater than $50 \%$. This was done so that delineation results could be evaluated with minimal influence of detection error. Crown 
diameters for the automated delineations were measured in the same orientations as the field measurements and averaged. An automated method for crown diameter measurement was developed, which used the bounding rectangle to determine the crown centroid and then calculated the distance from the centroid to the polygon boundary.

\subsection{Aggregated Assessment of Detection-Delineation and Tree Height}

The results were also evaluated as aggregated estimates for the entire transect as this reflects how they would most likely be used in regeneration assessment and management. Aggregate estimates of stem density and average crown diameter extracted from the detected-delineated crowns, as well as tree height modeled from crown diameter were compared with field measurements to determine percent error. Height was estimated using the delineated crown diameter in a linear equation derived from the field data $\left(\right.$ Height $=1.34^{*}$ Crown_Diameter $\left.+28, \mathrm{r}^{2}=0.81, \mathrm{SE}=25 \mathrm{~cm}\right)$. Detection, delineation and tree height aggregate estimates were compared for all trees and for the 30 and $60 \mathrm{~cm}$ crown size thresholds.

\subsubsection{Results}

\subsubsection{Visual Assessment}

Figure 5.3.4 shows the example plots that were selected for visual assessment. It should be noted that the blurry nature of the imagery was due to strong vibrations at the end of the boom that housed the camera. In all cases, it was apparent that crowns with strong brightness valleys were well delineated. Omission error occurred for small trees (A) and 
trees growing in close proximity or in large clumps (B). Commission error was less

frequent in the example plots, with errors caused by low-lying ground vegetation (C) and

within crown brightness variability (D). Woody stem competition did not significantly

hide trees from the sensor in this leaf-off imagery, but automated delineations do appear

to be smaller than the field measured ellipses for the plot shown in the bottom row of

Figure 5.3.4. Figure 5.3.5 shows sample areas for each transect at a scale suitable for a

broad overview of the results.

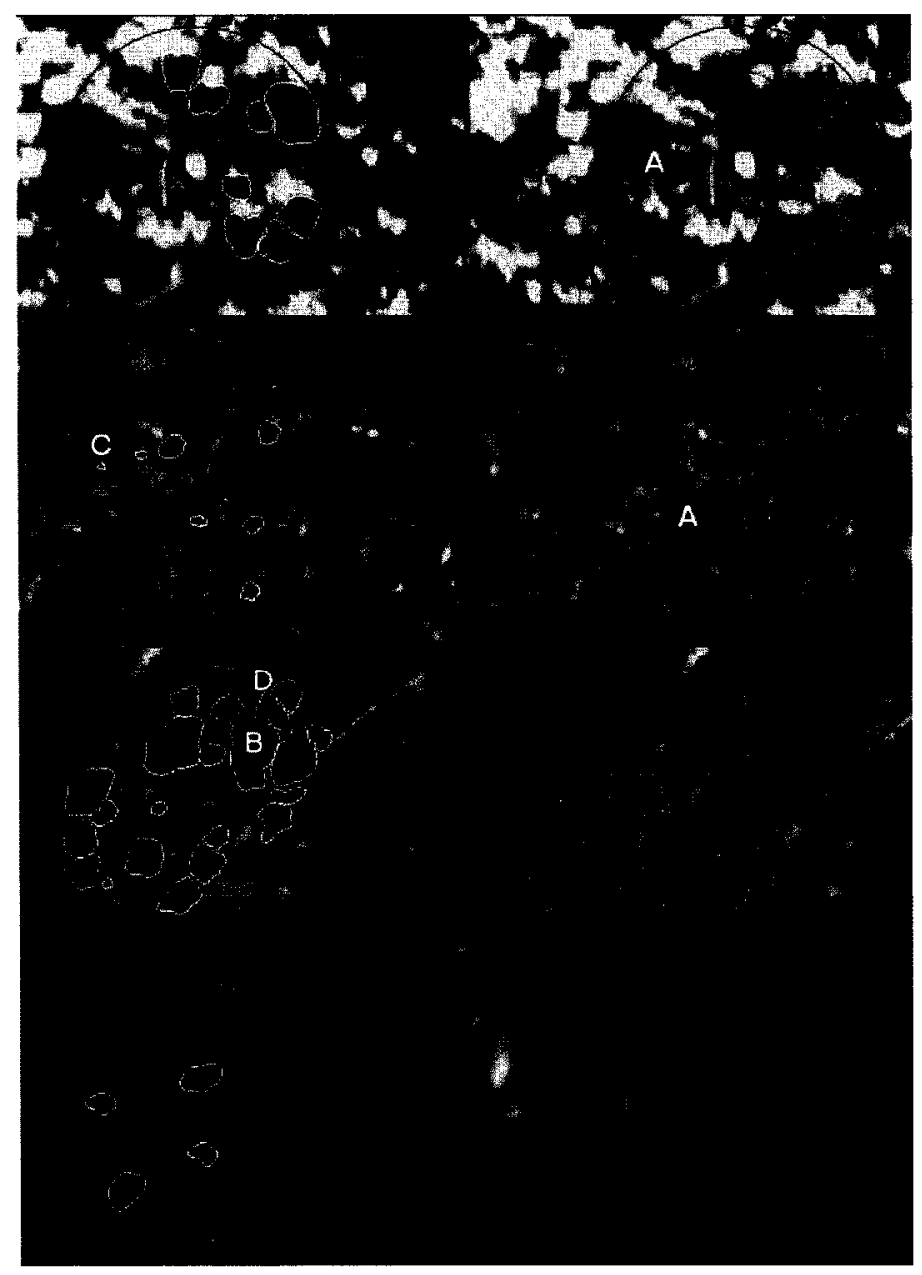

Figure 5.3.4: Example results for selected plots. Yellow - automated crown delineations, green - field located and measured crown ellipses. The rows represent site conditions as follows: Row 1: plot showing typical conditions with a few large and small trees, some being in close proximity to one another; Row 2: plot with mostly small trees, crown diameters being from 10-40 cm; Row 3: plot with a high degree of tree clustering; Row 4: plot with a high degree of woody stem competition. Common detection errors are marked A, B, C, and D as described in text. 


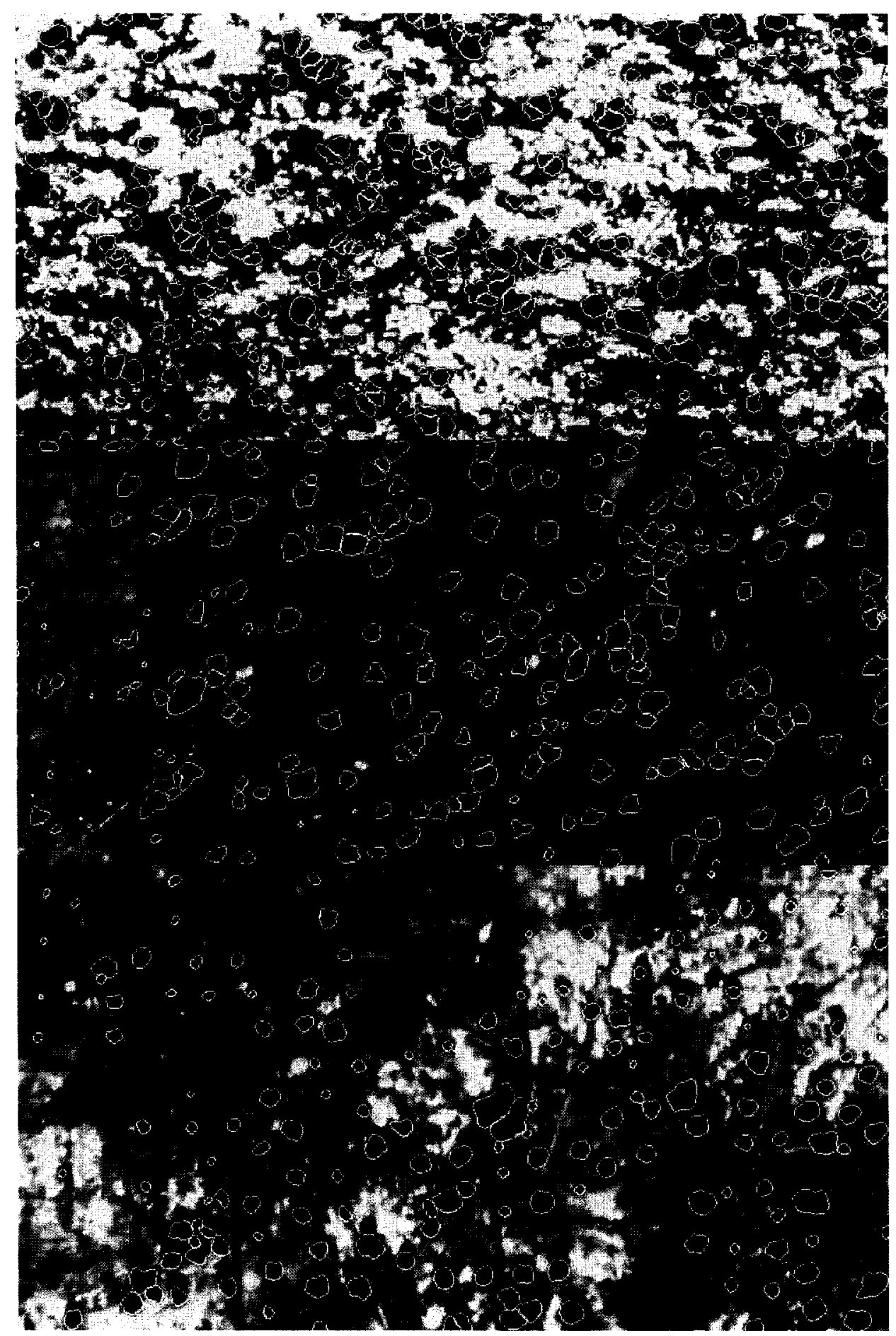

Figure 5.3.5: Example overview images of detection-delineation results for the three transects. Top - Low, middle - Moderate, and bottom - High transects. 


\subsubsection{Detection Accuracy}

The empirically determined optimal smoothing factor for each site was found to be 3,2 , and $1.5 \sigma$, giving $A I$ values of $72.8,66.4$ and $44.9 \%$ for the High, Moderate and Low transects, respectively (Table 5.3.1). These results are considered to be the best obtainable for this imagery and detection algorithm because the image scale has been optimized to achieve the highest agreement with the field data. In practice, however, field data would not typically be abundant enough to conduct such an analysis.

Consequently, the scale selection step described previously was implemented as part of the overall detection-delineation algorithm, resulting in optimal smoothing factors of 2.4 , 2, and 1.6 for the same transects. With these smoothing intensities, accuracies are within $3 \%$ of the empirically determined optima (Table 5.3.2, all trees), indicating that the visual based $\sigma$ selection method performed well. The poorest results were observed for the Low transect due to high omission error. Omission error for this transect was almost double that of the other two transects. Absolute commission error was largest for the Moderate transect, but relative to the total number of field counted trees, the High transect suffered the most from commission error. 
Table 5.3.1: Detection accuracy with increasing Gaussian smoothing intensity. Values are tree counts unless specified otherwise.

\begin{tabular}{|lcccccc|}
\hline $\begin{array}{l}\text { Transect } \\
\text { and Plots }\end{array}$ & $\begin{array}{c}\text { Smoothing } \\
\text { Factor }(\boldsymbol{\sigma})\end{array}$ & Omission & Commission & Correct & Correct \% & AI \% \\
\hline Low & 1.0 & 113 & 66 & 143 & 55.9 & 30.1 \\
Low & $\mathbf{1 . 5}$ & $\mathbf{1 2 5}$ & $\mathbf{1 6}$ & $\mathbf{1 2 9}$ & $\mathbf{5 0 . 4}$ & $\mathbf{4 4 . 9}$ \\
Low & 2.0 & 135 & 9 & 121 & 47.3 & 43.8 \\
Low & 2.5 & 143 & 5 & 113 & 44.1 & 42.2 \\
Low & 3.0 & 151 & 5 & 105 & 41.0 & 39.1 \\
Low & 3.5 & 152 & 5 & 104 & 40.6 & 38.7 \\
\hline Mod & 1.0 & 43 & 87 & 222 & 83.8 & 50.9 \\
Mod & 1.5 & 55 & 36 & 210 & 79.2 & 65.7 \\
Mod & 2.0 & $\mathbf{6 4}$ & $\mathbf{2 5}$ & $\mathbf{2 0 1}$ & $\mathbf{7 5 . 8}$ & $\mathbf{6 6 . 4}$ \\
Mod & 2.5 & 71 & 21 & 194 & 73.2 & 65.3 \\
Mod & 3.0 & 75 & 19 & 190 & 71.7 & 64.5 \\
Mod & 3.5 & 78 & 19 & 187 & 70.6 & 63.4 \\
\hline High & 1.0 & 11 & 46 & 103 & 90.4 & 50.0 \\
High & 1.5 & 14 & 33 & 100 & 87.7 & 58.8 \\
High & 2.0 & 14 & 19 & 100 & 87.7 & 71.1 \\
High & 2.5 & 16 & 18 & 98 & 86.0 & 70.2 \\
High & $\mathbf{3 . 0}$ & $\mathbf{1 6}$ & $\mathbf{1 5}$ & $\mathbf{9 8}$ & $\mathbf{8 6 . 0}$ & $\mathbf{7 2 . 8}$ \\
High & 3.5 & 17 & 15 & 97 & 85.1 & 71.9 \\
\hline
\end{tabular}

Table 5.3.2: Detection accuracy for all trees and for trees with crown diameters greater than 30 and $60 \mathrm{~cm}$. Values are tree counts unless specified otherwise.

\begin{tabular}{|cccccccc|}
\hline Transect & Min Size & Omission & Commission & Correct & Total & Correct \% & AI \% \\
\hline Low & All & 125 & 10 & 129 & 256 & 50.4 & 47.3 \\
Low & $>30$ & 44 & 7 & 124 & 168 & 73.8 & 69.6 \\
Low & $>60$ & 16 & 2 & 78 & 94 & 83.0 & 80.9 \\
\hline Mod & All & 64 & 25 & 201 & 265 & 75.8 & 66.4 \\
Mod & $>30$ & 22 & 13 & 186 & 208 & 89.4 & 83.2 \\
Mod & $>60$ & 7 & 4 & 118 & 125 & 94.4 & 91.2 \\
\hline High & All & 15 & 19 & 99 & 114 & 86.8 & 70.2 \\
High & $>30$ & 7 & 7 & 97 & 104 & 93.3 & 86.7 \\
High & $>60$ & 2 & 5 & 67 & 69 & 96.7 & 90.0 \\
\hline
\end{tabular}

To more fully understand the sources and magnitude of detection errors, omission and commission errors were visually evaluated and classified as to their most likely source. Figure 5.3.6 shows examples of errors of omission: A) a large crown overtops a smaller crown, hiding it from the sensor view, B) two proximal crowns result in a weak or non- 
existent between-crown brightness valley, C) a very small isolated crown $(15 \mathrm{~cm}$ diameter) with a signal too weak to be detected, D) a small crown (30 $\mathrm{cm}$ diameter) with a weak signal that is further reduced by the presence of competing vegetation. Errors of commission are also shown in Figure 5.3.6: E) low-lying non-crop vegetation detected and delineated as several tree crowns, and F) branch clusters in a crown detected and delineated as two crowns.

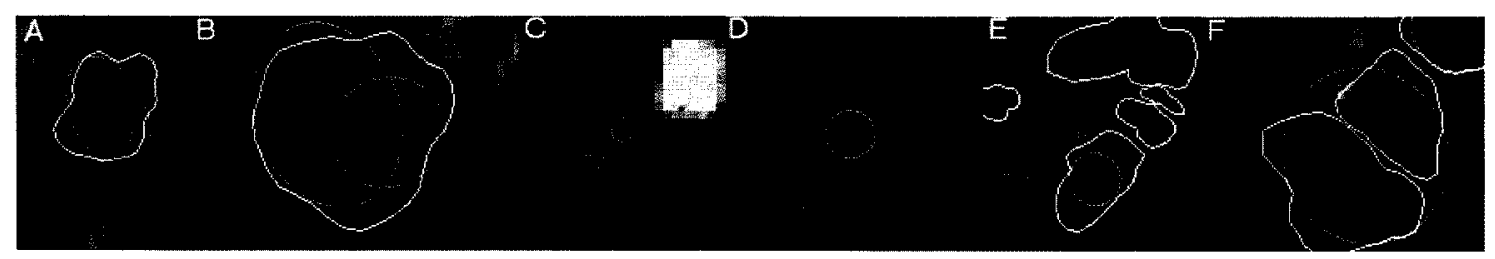

Figure 5.3.6: Examples of detection error sources. Green segments are field measured reference ellipses. Yellow are automated delineation results. A-D show omission errors and E-F show commission errors as described in text.

The largest source of detection omission error was the presence of tree crowns smaller than could be detected with this pixel size and the selected smoothing factors, but it was only greater than other error sources for the Low transect (Table 5.3.3). Adjacent trees in close proximity were the second largest error source. Additional omission errors due to small trees being overtopped by larger conifers occurred in both the Low and Moderate transects. These transects are younger than the High transect so sufficient competition between conifers and from overtopping woody vegetation has not yet resulted in mortality of these small trees. This has produced a more clumped spatial arrangement of conifers in the Low and Moderate sites than in the High site. The average diameter of omitted tree crowns was of $32 \mathrm{~cm}(\mathrm{sd}=26 \mathrm{~cm})$. Woody competition did not significantly affect omission error. 
Table 5.3.3: Counts of detection error category and source.

\begin{tabular}{|l|cccc|cc|}
\hline Transect & \multicolumn{3}{|c|}{ Omission Errors } & \multicolumn{2}{c|}{ Commission Errors } \\
\cline { 2 - 7 } & $\begin{array}{c}\text { Adjacent } \\
\text { overtopped } \\
\text { tree }\end{array}$ & $\begin{array}{c}\text { Adjacent trees } \\
\text { in close } \\
\text { proximity }\end{array}$ & Small tree & $\begin{array}{c}\text { Small tree with } \\
\text { competition }\end{array}$ & $\begin{array}{c}\text { Branch } \\
\text { pattern }\end{array}$ & $\begin{array}{c}\text { Low ground } \\
\text { vegetation }\end{array}$ \\
\hline Low & 7 & 35 & 83 & 0 & 6 & 4 \\
Mod & 16 & 23 & 23 & 5 & 4 & 21 \\
High & 0 & 5 & 5 & 5 & 7 & 12 \\
Total & $\mathbf{2 3}$ & $\mathbf{6 3}$ & $\mathbf{1 1 1}$ & $\mathbf{1 0}$ & $\mathbf{1 7}$ & $\mathbf{3 7}$ \\
\hline
\end{tabular}

Commission errors due to low-lying vegetation were greater than those due to within crown branching. They typically consisted of small segments generated where a tree did not exist or, in the case of within crown brightness variability, adjacent small and large segments generated for a single crown. The tree-matching algorithm used to evaluate accuracy takes the largest automated segment to represent the field-determined crown segment and the smaller automated segment(s) are taken as commission errors. The average diameter of commission error segments was $50 \mathrm{~cm}(\mathrm{sd}=33 \mathrm{~cm})$.

Because detection errors were caused by small trees being missed or by the generation of small but false segments in the processing results, substantial improvement is possible by thresholding the crown sizes used for validation. Increasing the minimum crown size to $30 \mathrm{~cm}$ dramatically improved results for all three transects, with the greatest increase in $A I(22 \%)$ occurring for the Low transect (Table 5.3.2). At this size threshold, two of the three sites have $A I>80 \%$. Increasing the minimum crown size to $60 \mathrm{~cm}$ again increased accuracy, but at a slower rate as the accuracies approached $100 \%$. 


\subsubsection{Delineation Accuracy}

For all matched crowns, the MAE error for delineation ranges from 15-23\% (Table 5.3.4). RMSE\% values are higher (22-35\%), showing that it is more affected by extreme values in the data. Results for crowns matched as one automated segment to one field reference ellipse (i.e., 1:1) show considerable improvement with $\mathrm{MAE} \%$ values ranging from $13-17 \%$. For these trees, strong linear relations were found with $r^{2}$ values ranging from $0.76-0.87$ and slopes close to 1 , but with varying magnitude and direction of offsets (Figure 5.3.7). Larger crowns in the imagery tended to be underestimated by the delineation algorithm. This error is more prevalent for the High transect and explains why it had the poorest delineation results. For the Low transect there was a slight overestimation of small crowns less than about $50 \mathrm{~cm}$ in diameter.

Table 5.3.4: Summary statistics for delineation accuracy.

\begin{tabular}{|c|c|c|c|c|c|c|c|}
\hline Transect & $\mathbf{r}^{2}$ & Slope & Offset & $\begin{array}{c}\text { MAE } \\
(\mathbf{c m})\end{array}$ & $\begin{array}{c}\text { MAE } \\
\%\end{array}$ & $\begin{array}{c}\text { RMSE } \\
\text { (cm) }\end{array}$ & RMSE\% \\
\hline Low & 0.64 & 1.1 & -9.5 & 16.1 & 20 & 22.7 & 28.8 \\
\hline Low I:I & 0.73 & 1.12 & -78 & 10.5 & 14.15 & 14.5 & 20.6 \\
\hline Mod & 0.76 & 1 & 2.1 & 10.5 & 15 & 15.1 & 21.7 \\
\hline Modl1 1 & 0.82 & 101 & 0.05 & 9 & 13.3 & 12.8 & 18.9 \\
\hline High & 0.6 & 1.1 & -1.6 & 18.7 & 22.7 & 28.7 & 34.9 \\
\hline VIgl, I: & 0.75 & 1.95 & 1.07 & 13.2 & 17.1 & 17.6 & 22.7 \\
\hline
\end{tabular}



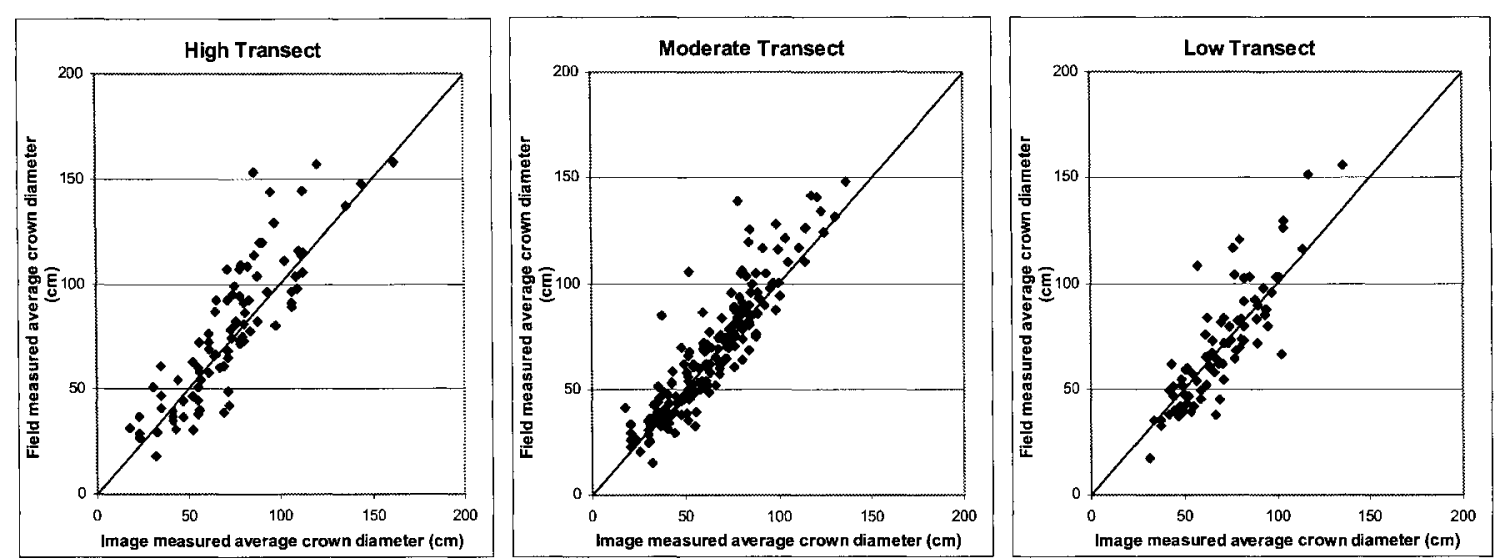

Figure 5.3.7: Scatterplots of field measured vs. image measured average crown diameter for trees matched as one automated segment to one reference ellipse (i.e. 1:1). Solid line represents $y=x$.

The average plot error, in terms of the difference between image and field measured crown diameter showed a weak relation with the number of competing woody stems within the plot (Figure 5.3.8). The relation was stronger when only woody stems above $2.5 \mathrm{~m}$ in height were counted. This was due to the effect of the increased overtopping of larger trees, which tended to obscure the conifer crown boundaries to a greater degree. Although there does appear to be a relation, it does not consistently produce errors that are larger than those caused by non-competition factors (i.e. spread of errors where little or no competition was present). 

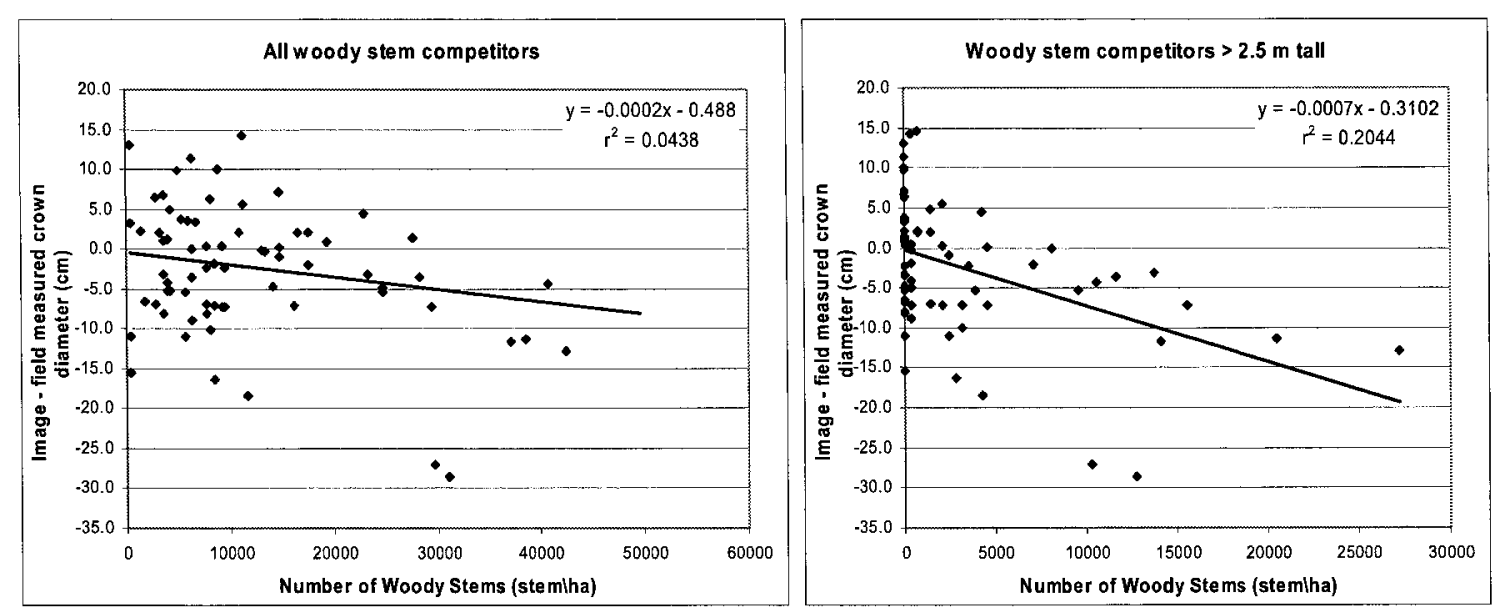

Figure 5.3.8: Scatterplots of the difference between image and field measured mean plot crown diameter and the stem density of woody competition.

\subsubsection{Aggregated Detection-Delineation Errors}

In aggregation of the results for each transect, errors were lower than the individual-tree comparisons (Table 5.3.5). The best results were found for the $30 \mathrm{~cm}$ crown size threshold. Increasing the threshold to $60 \mathrm{~cm}$ improved detection, but errors for crown diameter and tree height increased due to the greater error in delineation of larger trees. For crown diameter and height estimates, exclusion of trees less than $30 \mathrm{~cm}$ produced very high accuracy for the Low transect and good accuracy for the others. As these results reveal, the improvements due to aggregation are strongly influenced by the bias in the individual estimates. This is evident for the crown diameter comparisons, which, on an individual basis, resulted in larger crown sizes being underestimated, leading to larger error for aggregated comparisons with larger crown sizes. 
Table 5.3.5: Transect mean and percent difference measures between image and field measured tree density, crown diameter, and height.

\begin{tabular}{|c|c|c|c|c|c|c|c|c|c|c|}
\hline \multirow{2}{*}{$\begin{array}{c}\text { Trans } \\
\text {-ect }\end{array}$} & \multirow{2}{*}{$\begin{array}{c}\text { Min } \\
\text { Crown } \\
\text { Size } \\
\end{array}$} & \multicolumn{3}{|c|}{$\begin{array}{c}\text { Tree Density (stems ha }{ }^{-1} \text { ) } \\
\%\end{array}$} & \multicolumn{3}{|c|}{$\begin{array}{c}\text { Crown Diameter (cm) } \\
\%\end{array}$} & \multicolumn{3}{|c|}{ Tree Height (cm) } \\
\hline & & Image & Field & $\begin{array}{c}\% \\
\text { Error }\end{array}$ & Image & Field & $\begin{array}{c}\% \\
\text { Error }\end{array}$ & Image & Field & $\begin{array}{c}\% \\
\text { Error }\end{array}$ \\
\hline Low & All & 2459 & 4529 & -45.7 & 75.4 & 55.0 & 37.1 & 127.7 & 93.0 & 37.3 \\
\hline Low & $>30$ & 2318 & 2972 & -22.0 & 75.4 & 76.1 & -0.9 & 127.7 & 125.8 & 1.5 \\
\hline Low & $>60$ & 1415 & 1663 & -14.9 & 88.3 & 95.6 & -7.6 & 145.3 & 151.8 & -4.3 \\
\hline Mod & All & 3999 & 4689 & -14.7 & 63.5 & 59.5 & 6.7 & 112.4 & 110.0 & 2.2 \\
\hline Mod & $>30$ & 3521 & 3680 & -4.3 & 67.7 & 71.1 & -4.8 & 117.9 & 128.0 & -7.9 \\
\hline Mod & $>60$ & 2158 & 2212 & -2.4 & 83.5 & 88.4 & -5.5 & 150.6 & 140.0 & 7.6 \\
\hline High & All & 2088 & 2017 & 3.5 & 71.2 & 77.0 & -7.5 & 123.4 & 138.0 & -10.6 \\
\hline High & $>30$ & 1831 & 1833 & 0.1 & 76.8 & 83.3 & -7.8 & 131.0 & 147.0 & -10.9 \\
\hline High & $>60$ & 1261 & 1221 & 3.3 & 89.4 & 102.0 & -12.4 & 148.0 & 170.0 & -12.9 \\
\hline
\end{tabular}

\subsubsection{Discussion}

\subsubsection{Detection Accuracy}

On an individual tree basis, moderate accuracies for detection of 6 to $300 \mathrm{~cm}$ tall conifers $(A I=48-70 \%)$ were produced using the algorithm implemented on $6 \mathrm{~cm}$ pixel size digital camera imagery. Application of minimum crown diameter thresholds of $30 \mathrm{~cm}$ and $60 \mathrm{~cm}$ improved detection accuracies to $70-87 \%$ and $81-91 \%$, respectively. This was because most errors of omission and commission were linked to small trees or low lying non-tree vegetation. Depending on management and sampling objectives, the omission of such small trees may be inconsequential. This tree size influence was also seen in the aggregated transect level comparison, where accuracies were significantly better for crowns larger than $30 \mathrm{~cm}$. The Low competition transect, which had many small crowns that could not be detected, produced the greatest improvement in accuracy when the small trees were excluded. 
The high omission error observed in the Low transect was due to two factors. First, the crown size distribution of the Low transect was skewed towards smaller crowns, of which there were many. Second, the preprocessing step used to extract the crowns (unsupervised cluster labeling) was biased towards missing many of these small crowns. The bias resulted from the abundance of low-lying vegetation, particularly mosses in this site, which could not be adequately separated from the small crowns in creation of the crown mask. Thus, omission error due to small crowns was preferred over commission error due to low-lying vegetation in this transect. This same bias was applied when extracting crown pixels from the other two transects, but the resulting error was not as extreme because they did not have as many small crowns.

Additional means to improve detection of small trees include improvement of image quality and modification of data acquisition timing, data types, and image processing. The quality of imagery acquired for this study was poor relative to what was expected for $6 \mathrm{~cm}$ pixel size. Although the flight speed was low, the strong boom vibration caused significant image motion blurring. Either faster shutter speeds, andlor a more vibration isolated mount would reduce these effects and provide for more precise tree detection and crown boundary delineation. In terms of image timing, true leaf-off imaging cannot be considered a viable solution, as many mosses maintain their greenness throughout the year and many grasses were green within a few days of snowmelt. Such a narrow time period for image acquisition is too limiting for operational applications. Instead, data could be acquired in winter, however, snow depth would have to be monitored and acquisition would have to be carried out when snow has melted off crowns. If other data 
types can be considered, combined use of digital camera imagery and lidar would be advantageous, although costs associated with lidar systems can be high. Leckie et al. (2003b) compared detection-delineation results for both sensors in mature forest conditions and found that lidar data were particularly useful in more open forest conditions because a simple height threshold could be applied to remove false detection due to what was considered to be low-lying vegetation. Implementing an improved classification methodology is a potentially low cost alternative. Object-based classification, such as that available with the commercial package eCognition, may be used to separate low-lying vegetation from coniferous crowns. The advantage is that the spectral responses of objects such as small crowns and low lying vegetation that are generated by such a region- growing procedure are likely to be more distinct than the individual pixels contained in each object.

Omission errors caused by small trees and trees in close proximity as well as commission errors due to over-segmentation of trees with strong branching patterns are errors related to image scale. At scales with more detail there is an increased probability of correctly detecting small trees and trees in close proximity because the local valley brightness gradients used to separate crowns are more distinct. However, for large crowns, fine scales tend to retain distinct brightness valleys due to branches rather than crown boundaries, leading to false crown detection-delineation. Ideally, the appropriate scale for detecting a particular tree should be defined by the image characteristics in the local vicinity of the crown. Unfortunately, this is not a simple task. Pouliot and King (2005a) found that for conditions with variable tree size and spacing, the best detection results 
were obtained using a locally determined scale instead of a global scale. However, the local approach proved to be highly parameter intensive and therefore was not considered operationally useful, nor suitable for the present study. Further research in scaling for optimal feature extraction and other optimal detection approaches is needed to overcome this general limitation of segmentation methods.

\subsubsection{Delineation Accuracy}

In previous research using a more simplified algorithm under controlled tree spacing and competition conditions, Pouliot et al. (2002) found individual tree delineation accuracies for crowns detected as 1:1 to have RMSE $\%=17.9 \%$ for $5 \mathrm{~cm}$ pixel spacing imagery. In this study, under uncontrolled operational conditions with a more advanced algorithm, RMSE\% was $19-23 \%$. As identified in this study, Pouliot et al. (2002) also found that the large crowns were underestimated by the detection-delineation processing. For spatially aggregated results, Gougeon and Leckie (1999) compared average crown diameters for entire stands and found errors ranging from 7-9\%. In Pouliot et al. (2002) whole-plot error was $3 \%$. In this study, whole-transect error ranged from 7-37\% for all trees and $1-8 \%$ when only trees with a crown diameter greater than $30 \mathrm{~cm}$ were considered. The major difference between these studies, apart from the algorithms and image pixel sizes used, was the size and spacing variability of the trees, with much less variability in the studies presented by Gougeon and Leckie (1999) and Pouliot et al. (2002). In mature forests, Brandtberg and Walter (1998) did not find a significant relation between field and image measured crown diameters. Persson et al. (2002) reported a RMSE of $0.61 \mathrm{~m}$ using lidar data, but the average crown diameter was not 
reported and could only be inferred from graphs given in their study. Assuming an average crown diameter of $5 \mathrm{~m}$, their results are quite good with an RMSE of $\sim 12 \%$. The field and image measures were also linearly related $\left(r^{2}=0.58\right)$ and, like the results presented here, tended to slightly underestimate larger crowns.

Delineation error is heavily tied to detection error due to the effects of commission error for large crowns reducing the crown size estimates. Thus, the improvements identified for detection will improve the delineation results. Further improvement in delineation accuracy is also possible with sub-pixel processing. For the data used in this study, the 6 $\mathrm{cm}$ pixel size and selected smoothing factors could result in diameter estimate errors of $\sim 1$ to $6 \mathrm{~cm}$ depending on how the $6 \mathrm{~cm}$ grid of the acquired image data overlaid the tree crowns. For example, a mixed pixel with an area of $55 \%$ conifer and $45 \%$ soil would be seen as a crown pixel. If this pixel is used in the crown diameter estimate it would cause an over estimation of the crown diameter by $\sim 3 \mathrm{~cm}$ due to the mixed pixel effect. Thus, if two of these pixels are included in the diameter estimate, a maximum error of $\sim 6 \mathrm{~cm}$ could result. This suggests possible errors of up to $9 \%$ considering the average crown size for transects in this study was $65 \mathrm{~cm}$. Another important source of error was the field measurements, which, based on a re-measurement of 30 trees, showed the average absolute error to be $5 \mathrm{~cm}$. Thus, an additional $8 \%$ error was possible.

\subsubsection{Effect of Competition}

The effect of adjacent and overtopping woody stem vegetation on conifer detectiondelineation accuracy was small relative to other sources. Small crowns in plots with or 
without high competition were difficult to accurately detect and there were insufficient data on small crowns in high competition areas to conclusively assess competition effects. Larger crowns (i.e. $>30 \mathrm{~cm}$ ) were detected successfully with competition present. Delineation error was more clearly affected by the presence of competing vegetation and tended to generally reduce crown size estimates. However, in many cases it appeared that competition was a less important factor for delineation error than other error sources such as image scale and spatial arrangement of trees. The use of plot averages to represent competition intensity rather than individual measurements was a significant limitation of this analysis. These measurements were made on a plot level in order to satisfy several analysis objectives. Individual measurements would have been preferred, as there was considerable variability in tree size, spacing, and spatial arrangement of both conifers and woody competition stems within the plots. The presence of this variability reduces the utility of the mean plot measures to be used for comparisons amongst plots. Despite these limitations, the results do suggest that competing vegetation is potentially less important than other sources of error.

\subsubsection{Operational Considerations}

Image data quality plays an important role in both detection and delineation. Factors such as sensor view angle, sun elevation, and topography have a significant effect on the radiometric and geometric properties of the tree crowns. Using simulated imagery of mature Eucalyptus forests, Culvenor (2000) showed that detection accuracy performance was best with small off nadir view angles (i.e., $<15^{\circ}$ ) using backscattered as opposed to forward scattered image data, and higher solar zenith angles. To reduce optical and 
illumination-view angle (i.e. bidirectional) effects, imagery with narrow view angles can be acquired with a high degree of overlap and used in subsequent mosaic generation. The effect of topography has not been widely researched. Culvenor et al. (2000) found that the effects of topography depended on the incident radiation relative to the topographic slope and aspect. At the tree level, differences in illumination due to topography cause differences in mutual shading of crowns and the brightness response of the background. At a coarser scale, topography produces shaded and sunlit slopes that make it difficult to effectively extract an accurate crown mask. More research is needed to develop appropriate means to reduce both crown level and terrain level topographic brightness effects on detection-delineation accuracy. Topography also affects pixel size, which in turn can impact crown size estimates, but this effect could be minimized through orthorectification if a suitable DEM is can be derived.

With the image pixel size used in this study, the proposed methodology would most likely be used in a strip-sampling approach to acquire greater sample coverage than could be obtained through field surveys. Such a methodology provides a potentially lower cost means to increase sample coverage, access remote areas, and retain a permanent visual record of the site conditions. An in-depth economic analysis is beyond the scope of this study, but eventually will have to be performed. Here the major cost was the use of the helicopter, billed at $\$ 1200$ hour $^{-1}$. Alternatively, fixed wing aircraft could be used at lower cost, but a camera with capability for higher shutter speeds than those of this study would be required due to the associated increased flying speed. In comparison to field 
surveys, this approach provides the means to dramatically increase sample coverage that cannot be achieved through field sampling. Further, the collected data can be used in other applications in addition to regeneration inventory.

\subsubsection{Conclusion}

An automated tree detection-delineation algorithm developed for monitoring regenerating forest conditions was presented and evaluated. Detection accuracy was moderate when all trees were considered, and improved significantly to acceptable levels when very small trees (crown size $<30 \mathrm{~cm}$ ) were excluded. The most significant factors found to affect detection were small trees, trees close together such that little or no distinct brightness valley was evident between them, and the presence of low-lying vegetation. Crown delineation absolute error was $<23 \%$ of field measured crown diameter, and large tree crown diameters were often underestimated. The presence of competing woody vegetation did not have a strong influence on detection and had only a small effect on delineation accuracy, with increasing competition reducing crown size estimates slightly. If applied to narrow view angle imagery with a small enough pixel size relative to the crown sizes that are required to be detected and measured, this algorithm could complement or replace field-based regeneration surveys.

\subsubsection{Acknowledgements}

This research was funded by grants to D. King and scholarships to D. Pouliot from the Natural Sciences and Engineering Research Council of Canada. The contributions from 
Wayne Bell, Mark Lindsay, Duncantech Ltd., Wiskair Ltd., and Buchanan Forest

Products Ltd. to this research are also much appreciated. 


\title{
5.4 LARGE AREA OPERATIONAL EVALUATION OF AUTOMATED TREE DETECTION AND DELINEATION USING AERIAL PHOTOGRAPHS
}

Citation: The following is adapted from - Pouliot, D.A., and D.J. King (2004). Fallingsnow ecosystem study aerial photography: automated tree detection-delineation and vegetation classification. Report produced under the Collaborative Research Agreement Regarding A Joint Living Legacy Trust Grant, Between The Upper Lakes Environmental Research Network and Carleton University. 20 pp.

\begin{abstract}
An automated tree detection and delineation algorithm was evaluated for a large area operational application using leaf-off 1:3000 and 1:5000 colour infrared aerial photography of 15 year old regenerating forest conditions. Larger scale (1:500) photography and field measurements of crown diameter served as reference data in validation of detection and crown measurement success. Errors in the number of conifers detected per plot were between 30 and $46 \%$ for plot level comparisons of tree density. These were reduced to $3.3-26.3 \%$ for block averages due to compensating effects of errors of omission and commission. Errors in average crown diameter per plot were $31-$ $36 \%$ while errors in average crown diameter per block were $3.8-8.3 \%$. Errors decreased with increasing proximity to the image centre due to reduced viewing geometry effects. A sample of manual detection results showed that similarly high errors to those of the automated method were obtained. The results of this study are encouraging despite limitations in the data used. It was necessary to use photographry as
\end{abstract}


part of the larger Fallingsnow Ecosystem project, but it was subject to strong sunanglelview-angle geometric and brightness distortions as well as topographic distortions and scanned pixel size limitations. In future work, reduced image view angle, increased image resolution, and use of a digital sensor with narrower spectral bands should improve the accuracies significantly.

\subsubsection{Introduction}

The Fallingsnow Ecosystem Study was a large multi-disciplinary research project established by the Ontario Ministry of Natural Resources in 1993 to evaluate the effects of manual, mechanical, and aerial herbicide release treatments on crop trees, non-crop vegetation and other selected environmental components. In 2003, a second phase of the research was implemented to assess the effects of these different management practices on vegetation as well biodiversity and wildlife. As part of the 2003 study, the objective of this research was to evaluate the potential of automated tree detection and delineation in characterizing the coniferous vegetation on these sites for long term monitoring. Aerial photography was acquired by the OMNR to match the data used for visual mapping of vegetation types in 1993 and because it is the standard low cost remote sensing data type used in operational forestry. This study served as a means to evaluate the detectiondelineation algorithm using wider angle photography than the digital camera imagery. The study was ideal for an operational test containing several different regeneration conditions resulting from the different vegetation control treatments over sizeable spatial extents. Thus, depending on the treatment applied, different tree sizes and levels of competing woody vegetation would be present to test algorithm performance. 


\subsubsection{Automated Tree Detection and Delineation}

Individual tree detection and delineation analysis identifies and segments tree crowns within an image for further analysis and information extraction. There has been considerable research in this area regarding mature forests (Gougeon, 1995a; Brandtberg and Walter, 1998; Pollock, 1998; Walsworth and King, 1998; Culvenor, 2000; Persson et al., 2002; and Brandtberg et al., 2003). However, very little work has been carried out in regenerating forest conditions where such analysis can provide important information needed to evaluate regeneration status.

Reliable tree detection can provide estimates of tree abundance and spatial pattern to verify if density objectives have been achieved. Delineation of individual trees provides for improved species identification through analysis of within-crown spectral data (Leckie et. al., 2003a), spatial data (Haddow et al., 2000), and crown shape (Brandtberg, 1998). Further, delineated crown dimensions can be used to model tree structural variables such as height, volume, or biomass that are useful to evaluate growth (Culvenor, 2000; Hayward et al., 2001).

In regenerating forests, algorithms developed by Gougeon and Leckie (1999) and Pouliot et al. (2002) have been evaluated. The results of these studies are encouraging, but in the case of Gougeon and Leckie (1999), the large pixel size did not allow for sufficient detection of smaller crowns in the imagery. In Pouliot et al. (2002), a detectiondelineation algorithm was evaluated in a controlled regeneration experiment of the OMNR at the Sault Ste. Marie arboretum. Since that time, the algorithm has been 
improved and has been tested in operational conditions using airborne digital camera imagery (Pouliot et al., 2005). For this project, the algorithm was applied to aerial photography acquired by the OMNR. The algorithm, data, data processing and detectiondelineation accuracy assessment are described below.

\subsubsection{Methods}

\subsubsection{Study Area}

The study was located in Fraleigh Township approximately $61 \mathrm{~km}$ southwest of Thunder Bay, Ontario $\left(45^{\circ} 10^{\prime} \mathrm{N}, 89^{\circ} 50^{\prime} \mathrm{W}\right)$. The trial was a Randomized Complete Block design, with 3 blocks and 5 treatments. The treatments applied (in 1993) were: 1) aerial application of Vision, a deciduous specific herbicide, 2) aerial application of Release also a deciduous herbicide, 3) manual release with brush-saws, 4) mechanical release with a Silvana SelectivelFord Versatile, a small tractor with a boom and saw system and 5) control (no treatment). In 1994, a sixth area in the uncut stand adjacent to each block was established and utilized for some components (Forest Ecosystem Classification (FEC) plots, songbird studies, photo-plots etc.). In each of the six treatment plots, eight $10 \times 10 \mathrm{~m}$ permanent FEC plots were randomly established. The total number of FEC plots was: 8 plots $\times 6$ treatments $\times 3$ blocks $=144$ plots. At the time of the study conifer species consisted of planted white spruce (Picea glauca) and naturally regenerating balsam fir (Abies balsamea), white pine (Pinus strobus) and white cedar (Thuja occidentalis). Tree height was greater than $2 \mathrm{~m}$ and average crown diameter was $\sim 129 \mathrm{~cm}$. Aspen (Populus tremuloides) and alder (Alnus spp.) were the main competitors. The topography was 
moderate, with some steep slopes. Figure 5.4.1 shows an oblique view of one of the cutblocks from across the valley and the forest conditions in 2003 in the Vision treated and control areas.

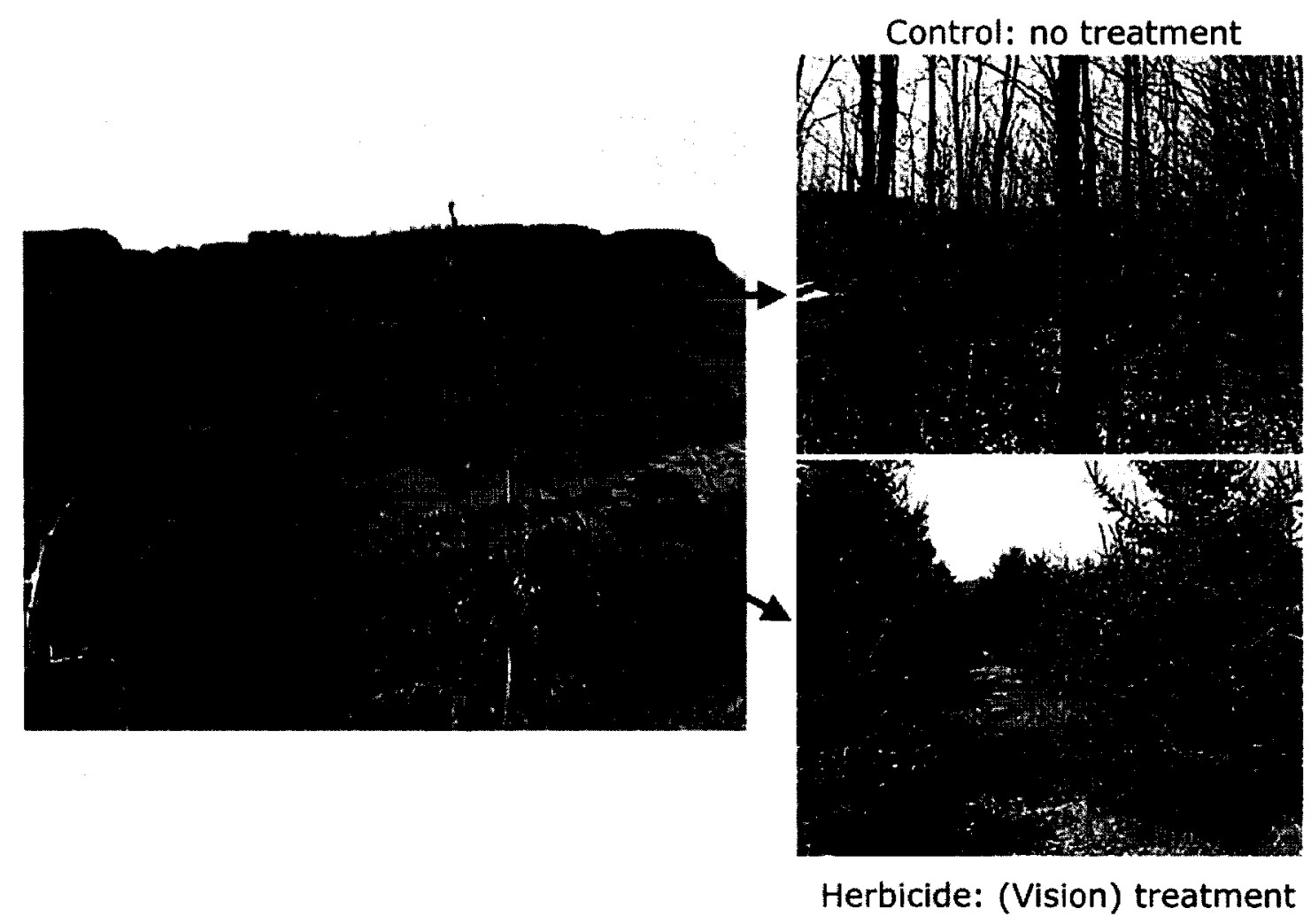

Figure 5.4.1: Example ground conditions.

\subsubsection{Leaf-Off Aerial Photography}

Twenty-three $\mathrm{cm}$ format aerial photography was acquired by the OMNR at 1:3000 and 1:5000 scales in early May 2003 under leaf-off conditions. The coverage of each photo was approximately $680 \times 680 \mathrm{~m}$ for the $1: 3000$ scale. The 1:3000 photographs were scanned at $510 \mathrm{dpi}$, giving an approximate ground pixel size of $15 \mathrm{~cm}$. For Block 2, extreme cloud shadow covered a large portion of the imagery, so the 1:3000 photos were 
not used. For this block, the 1:5000 photos, which were free from shadow, were scanned at $850 \mathrm{dpi}$ to give the same $15 \mathrm{~cm}$ pixel size as the 1:3000 imagery. Image to image registration for mosaic generation was carried out with 1st and 2nd order polynomial models resulting in an average RMSE of $<1 \mathrm{~m}$ for the control points. This is a high error considering the $15 \mathrm{~cm}$ pixel size, but it was mostly due to geometric distortion associated with the large view angles of the imagery. The viewing geometry caused substantial displacement of image objects leading to the high error and the need for higher order polynomial corrections. As an example, Figure 5.4.2 shows the same ground area but imaged at different view angles, clearly illustrating the effect of view angle on the appearance of image objects.

1:500 colour leaf-off photography was acquired for validation of detection-delineation results derived from the 1:3000 scale photos. However, pie plates used to mark the target plot corners were only visible in the 1:500 photos and not the 1:3000 photos. Consequently, plot locations could not be directly found in the 1:3000 photos. To overcome this problem, the 1:500 photos were scanned at $450 \mathrm{dpi}(3 \mathrm{~cm}$ resolution) and geo-referenced using the pie plate plot corner coordinates that had been measured in the field using differential GPS. Geo-referencing error ranged from $15-60 \mathrm{~cm}$. The 1:3000 mosaics were then aligned to these 1:500 photographs. Third order models were used, as there was considerable topographic variation in each block. The average RMSE error of the control points for all blocks was less than $3 \mathrm{~m}$. 


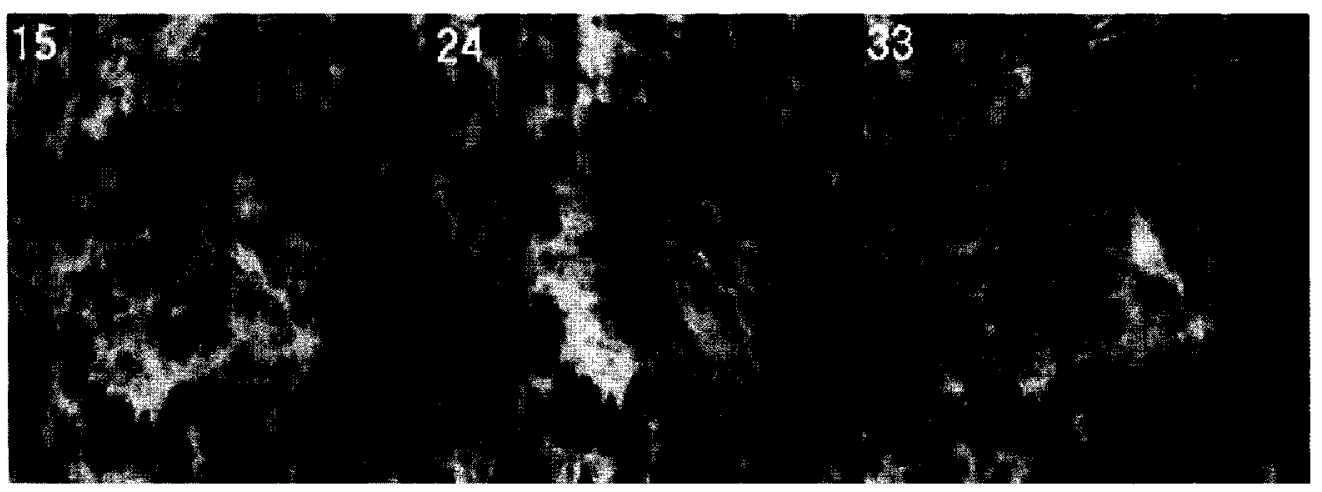

Figure 5.4.2: An area imaged at view angles of 15,24, and 33 degrees in different photos.

For validation, even better alignment between the two photo sets was required to accurately find plot boundaries in the 1:3000 photos, and to force individual trees in both photo sets to overlap as much as possible. This allowed matching of specific trees for error analysis. Consequently, an additional adjustment of the 1:500 photos to the 1:3000 photos was conducted, producing an RMSE of $<30 \mathrm{~cm}$. Vectors polygons of the plot boundaries and trees sampled for each block were then created from these photos. Field measurements were added as attributes to these vectors for use in validation.

\subsubsection{Field Data}

In each plot the total number of conifer stems within the plot was counted (by OMNR staff) and two sample trees were selected in the North-West and South-East corners of the plot for crown diameter measurement. Crown diameter was measured in the North-South and East-West directions. In total, data from 44 plots were used in detection validation, and 121 tree diameters were used in crown delineation validation. 


\subsubsection{Image-Based Conifer Detection-Delineation and Measurement}

A detection-delineation algorithm was designed that incorporates aspects of a previous algorithm developed by Pouliot et al. (2002) as well as other published algorithms. It has been optimized for forest regeneration conditions. Details can be found in Pouliot et al. (2005). The first step in the algorithm is to isolate vegetation pixels. This can be performed in numerous ways, but is typically accomplished by thresholding the imagery or by basic classification if multispectral imagery is available. The next step is to determine the optimal level of smoothing to apply to remove most of the radiometric image structure caused by noise or individual tree branches while maintaining good image detail. To do this, the relation between the number of crowns detected with increased smoothing is analyzed to determine a distinct break, which is taken as the optimal scale. This method was selected after several tests and is described in detail in Pouliot and King (2005). For this analysis, tree crowns are detected as local maxima brightness values in the imagery. After a suitable smoothing factor has been applied, initial crown detection and delineation is carried out using local gradient following (similar to a watershed segmentation technique). In this method, each pixel in the image is considered a seed and forced to climb the local upward brightness gradient until a local maximum point is reached. This seed pixel is then assigned to the clusterlsegment for that local maximum position. The initial segmentation results from the gradient following processing are highly dependent on the quality of the initial vegetation isolation, which in turn is dependent on the quality of the image data used and processing performed. For example, brightness variations due to bi-directional and optical light fall-off effects can lead to the removal of crown pixels or addition of non-crown pixels, depending on the 
criteria used to separate crowns from non-crown pixels. Consequently, a refinement process is implemented to minimize this dependence by locally assessing the boundaries for each crown based on the initial gradient following results. To do this, starting at each detected apex, a user specified number of transects around the candidate crown object are extended from the apex out to the local minimum boundary defined by the initial segmentation results plus one additional pixel. The transect data are then extracted and the maximum value in the first derivative is taken as the most suitable crown boundary position.

For this dataset, supervised maximum likelihood classification was used to extract vegetation pixels. Optimal smoothing using a Gaussian filter was found to be $2 \sigma$ and 36 transects were used for boundary refinement.

\subsubsection{Detection and Delineation Accuracy Evaluation}

Stem density was validated by comparing the number of trees detected in each plot by visual interpretation and by the automated algorithm with the number of stems counted on the ground. Some random error was expected due to the presence of borderline trees along the plot boundaries and was assumed to have only a slight effect on the results.

For delineation, the sampled trees in each plot were matched to the most overlapping segment from the automated delineation results. This was conducted for trees where only 1 automated segment overlapped the reference segment by greater than $15 \%$. This allowed diameter error due to delineation to be assessed independently from detection 
error. These paired comparisons were then used to calculate the mean absolute error (MAE), root mean square error (RMSE), and the relative MAE or RMSE error expressed as a percentage of the mean diameter value for the reference measurement. Manual (visual) delineations were also made in the imagery for seven randomly selected plots in each block. They were carried out using the image mosaics to provide direct comparison of measured crown diameters to the algorithm, which was applied to the same imagery.

\subsubsection{Results}

\subsubsection{Aerial Photography}

An example 1:3000 aerial photo is given in Figure 5.4.3. This image shows Block 4 in false colour $($ Near Infrared $=$ Red in image; Red $=$ Green in image; Green $=$ Blue in image). The photography was generally of adequate quality for visual interpretation but it suffered from saturation in tree crowns in all bands, tree geometry variations, and spatial brightness variations that are common with imagery of such a wide view angle and scale. These distortions impacted the detection and delineation accuracy as presented in the discussion.

\subsubsection{Automated Conifer Detection Results}

Table 5.4.1 shows the MAE and RMSE between field and automated image-based conifer counts per plot, calculated on a pair-wise basis (automated count plot 'x' vs. field count plot ' $x$ '). There is considerable difference between the MAE and RMSE error measures. The RMSE is more sensitive to extreme values resulting in larger error 
estimates. When averaged over each block, it can be seen that balancing errors of omission and commission produce block level errors of 5, 1 and 1 trees per plot, respectively. This translates into $5.2 \%, 8.3 \%$, and $3.3 \%$ average error over all plots for Blocks 2, 3, and 4, respectively.

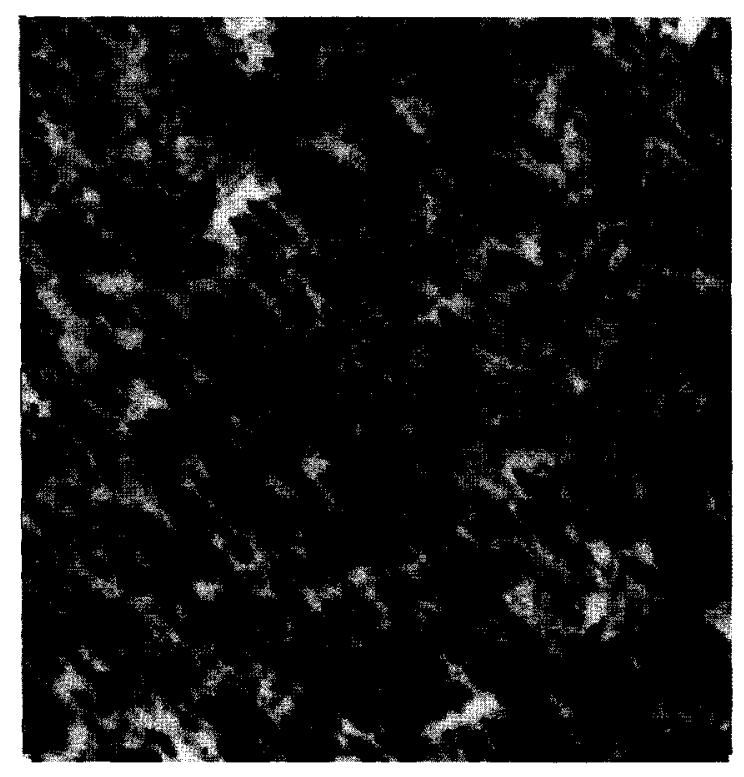

Figure 5.4.3: Example aerial photograph.

Table 5.4.1: Comparison of field and automated tree counts per plot.

\begin{tabular}{|c|c|c|c|c|c|c|c|}
\hline Block & $\begin{array}{c}\text { Number } \\
\text { of Plots }\end{array}$ & MAE & MAE\% & RMSE & RMSE\% & $\begin{array}{c}\text { Average } \\
\text { Automated } \\
\text { Count }\end{array}$ & $\begin{array}{c}\text { Average Field } \\
\text { Count }\end{array}$ \\
\hline $\mathbf{2}$ & 14 & 4.5 & 24 & 5.7 & 30 & 24 & 19 \\
\hline $\mathbf{3}$ & 15 & 2.7 & 23 & 3.2 & 27 & 13 & 12 \\
\hline $\mathbf{4}$ & 15 & 10.2 & 34 & 13.8 & 46 & 29 & 30 \\
\hline
\end{tabular}

Table 5.4.2 compares the MAE and RMSE pair-wise plot level error over all blocks for field, automated and visual (manual) tree counts. It can be seen that the manual imagebased counts are in error by the same amount as the automated algorithm when compared to field counts ( $48 \%$ vs. $50 \%$ RMSE). This shows that the imagery suffers from 
distortions and lack of resolution and cannot provide high plot-level absolute accuracy in tree counts. The manual and automated results are much closer to each other ( $25 \%$ RMS difference) but there is even a significant discrepancy between these two methods. The sources of this difference have not yet been determined. Further analysis below, however, reveals the sources of the differences between the automated and field count results.

Table 5.4.2: Comparison of the number of trees counted in each plot for field, automated and manual detection using a subset of 21 of 44 randomly selected plots.

\begin{tabular}{|c|c|c|}
\hline Comparison & RMSE & RMSE\% \\
\hline AutolField & 11 & 50 \\
\hline Man\Field & 11 & 48 \\
\hline Auto $\backslash$ Man & 6 & 25 \\
\hline
\end{tabular}

The primary factors limiting tree detection success in relation to field counts are the scanned image resolution, brightness variations with view angle, and geometric variations in crown shape and position with view angle. These are described further in the Discussion. For view angle, Table 5.4.3 and Figure 5.4.4 show the error in tree detection within zones of $0-10^{\circ}, 0-15^{\circ}, 0-20^{\circ}$, and over the whole image (all view angles). These data show that within smaller view angles the detection results are more accurate. The user of the data should decide what level of error is tolerable and then select the appropriate region around image nadir from which to extract detected crown counts. In Figure 5.4.5, an example of these zones overlaid on a block mosaic is given. 
Table 5.4.3: Automated tree count error (\# trees) for each view angle category.

\begin{tabular}{|l|cccc|}
\hline \multicolumn{4}{c|}{} & \multicolumn{4}{c|}{ View angle (degrees) } \\
\cline { 2 - 5 } MAE & All & $<\mathbf{2 0}$ & $<\mathbf{1 5}$ & $<\mathbf{1 0}$ \\
Standard dev. & 5.1 & 4.9 & 4.6 & 3.6 \\
Num. Cases & 4.1 & 5.0 & 4.7 & 3.3 \\
\hline
\end{tabular}

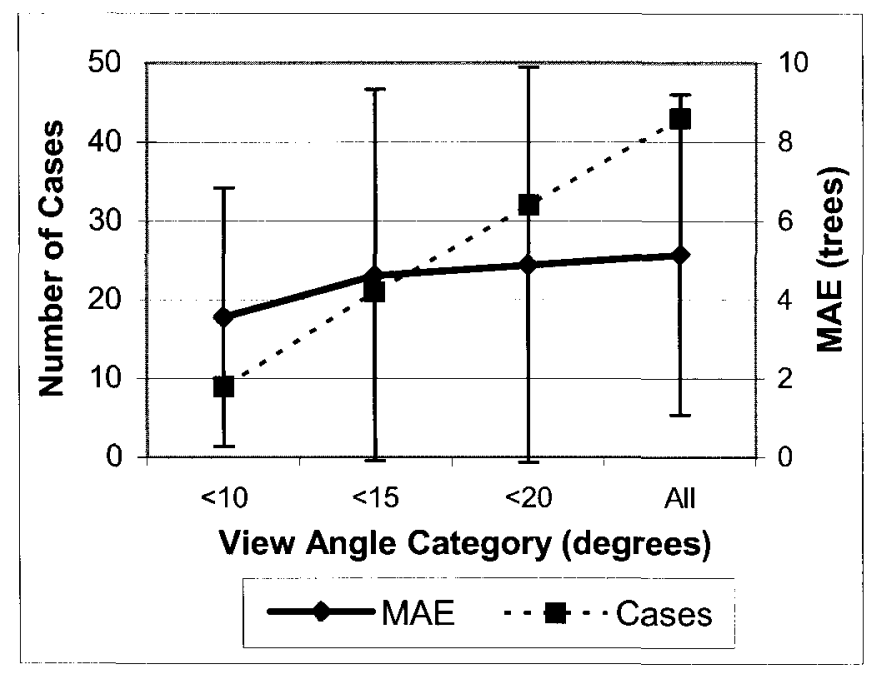

Figure 5.4.4: Automated tree detection error for sampled view angles. Error bars are \pm 1 standard deviation.
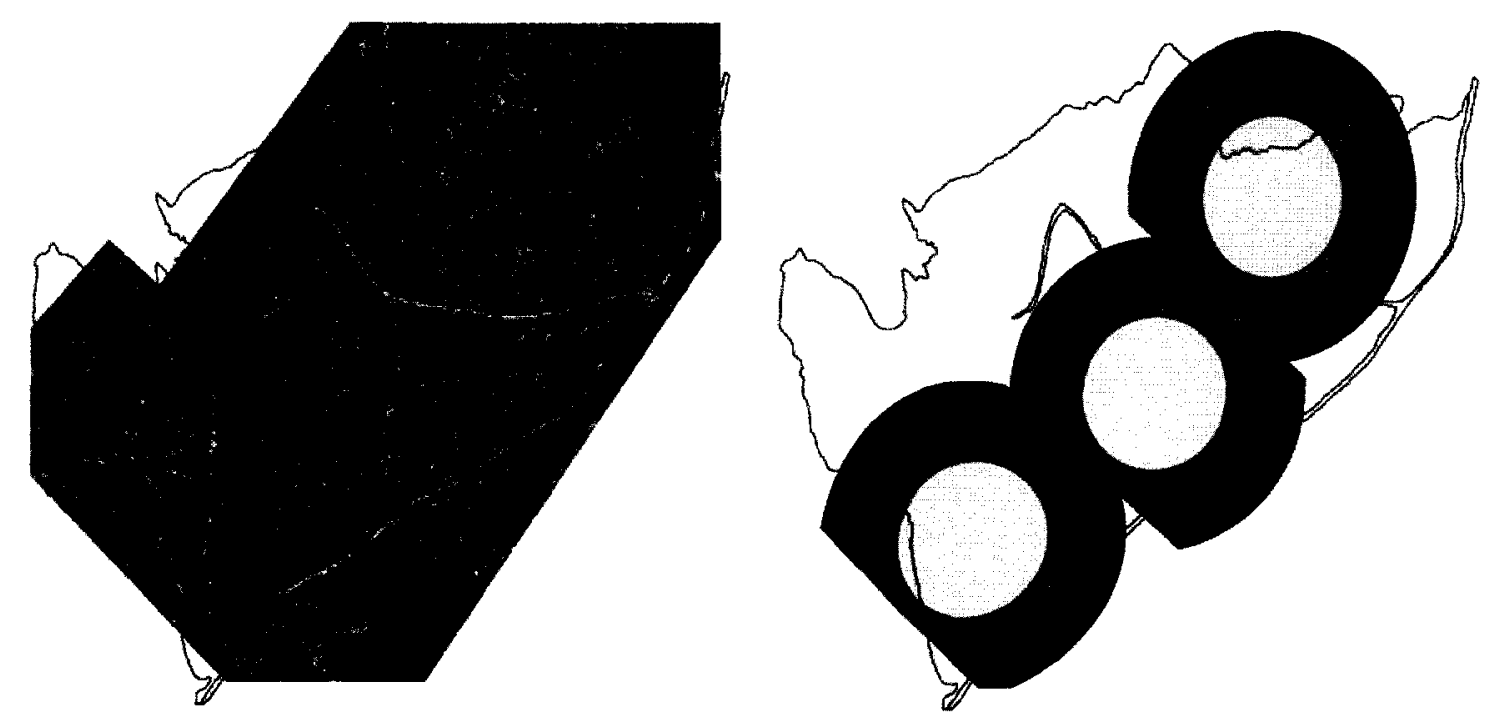

Figure 5.4.5: Block 4 image mosaic (left) and bounds of the $0-10,0-15$, and $0-20^{\circ}$ view angles in lightgrey, dark-grey and black, respectively, from each of its constituent photos (right). 


\subsubsection{Automated Conifer Crown Delineation Results}

Figure 5.4.6 shows crowns delineated using the automated algorithm. Table 5.4.4 shows the MAE and RMSE between average N-S\E-W crown diameter measured in the field and crowns delineated in the imagery using the automated algorithm. When averaged over each block, it can be seen that over-estimates and under-estimates balance somewhat to reduce block level average crown diameter errors to 14,10 , and $6 \mathrm{~cm}$, respectively. This translates to $8.3 \%, 5.4 \%$, and $3.8 \%$ average error overall all sample trees for Blocks 2, 3, and 4, respectively.

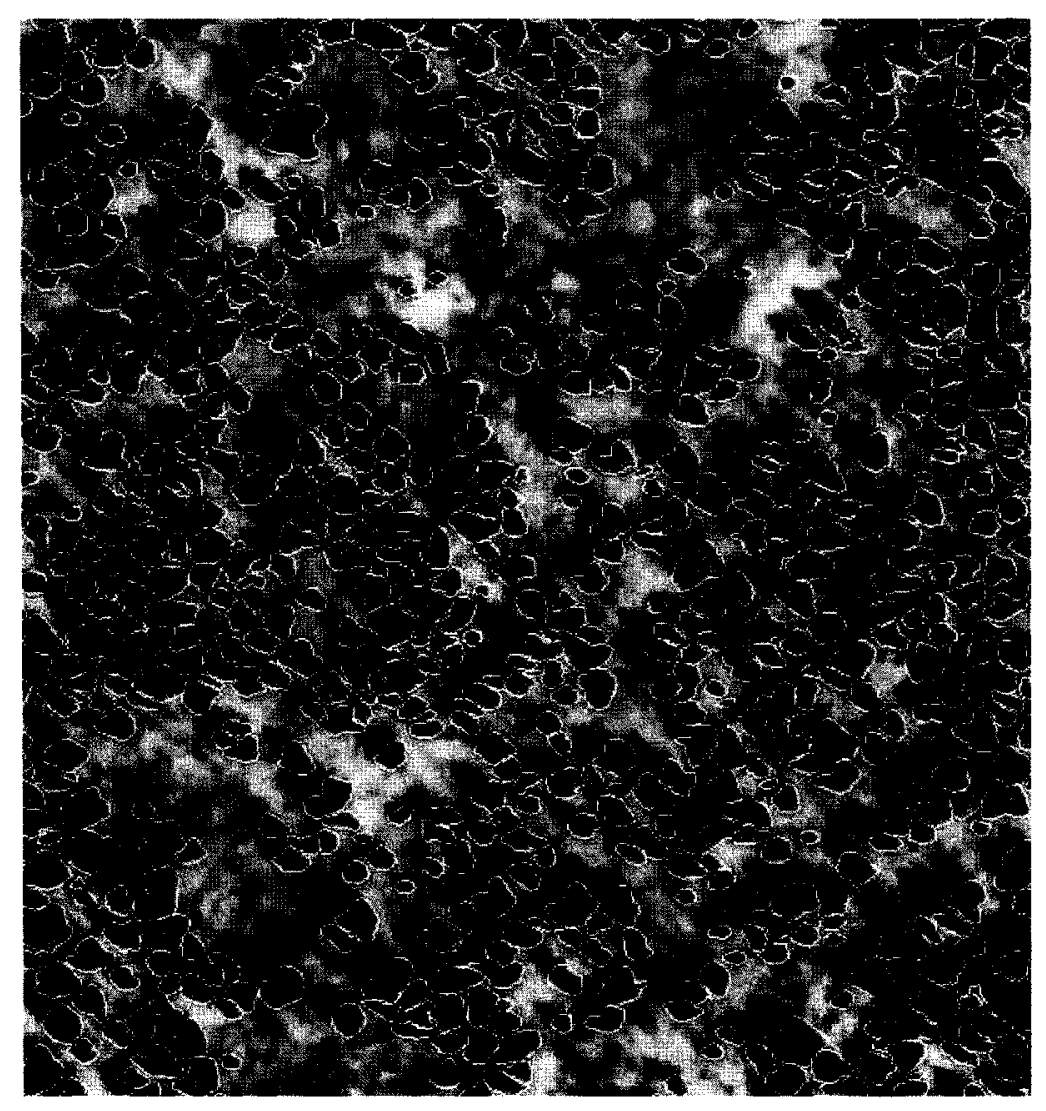

Figure 5.4.6: Overlay of automated delineation results on image data.

Table 5.4.5 gives a three-way comparison of average crown diameter measured in the field, from automated crown delineations and from manual delineations. Each of the 
methods produces about a $30 \%$ RMSE difference with each of the other methods. This shows that due to variations in delineations and how trees were measured in the field, error remains substantial when compared on a plot basis.

Table 5.4.4: Comparison of the average crown diameter measured in the field to that from automated crown delineation. Units of diameter, MAE and RMSE are $\mathrm{cm}$.

\begin{tabular}{|c|c|c|c|c|c|c|c|}
\hline Block & $\begin{array}{c}\text { Number } \\
\text { of Trees }\end{array}$ & MAE & MAE\% & RMSE & RMSE\% & $\begin{array}{c}\text { Average } \\
\text { Automated } \\
\text { Diameter (cm) }\end{array}$ & $\begin{array}{c}\text { Average Field } \\
\text { Diameter (cm) }\end{array}$ \\
\hline $\mathbf{2}$ & 31 & 43.9 & 26 & 59.1 & 35 & 183 & 169 \\
\hline $\mathbf{3}$ & 23 & 41.5 & 22.6 & 57 & 31 & 194 & 184 \\
\hline $\mathbf{4}$ & 28 & 41.9 & 26.2 & 57.6 & 36 & 166 & 160 \\
\hline
\end{tabular}

Table 5.4.5: Comparison of average crown diameter measured using the three methods for a subset of 21 of 44 randomly selected plots: field measurement, measurement from automated delineations, and measurement from manual delineations.

\begin{tabular}{|c|c|c|}
\hline Comparison & RMSE (cm) & RMSE\% \\
\hline Auto\Field & 55 & 30 \\
\hline Man\Field & 52 & 28 \\
\hline Auto\Man & 47 & 31 \\
\hline
\end{tabular}

Table 5.4.6 and Figure 5.4.7 show plot level delineation error vs. image view angle.

Delineation error increases more with view angle than did detection error (Table 5.4.3,

Figure 5.4.4). It is evident that due to image distortions (discussed below) and due to differences between image and field crown measurement that, if the user desires to match field measurements as close as possible, then only trees from the inner $10^{\circ}$ view angle should be used.

Table 5.4.6: Delineation error $(\mathrm{cm})$ for each view angle category.

\begin{tabular}{|l|cccc|}
\hline \multicolumn{4}{c|}{} & \multicolumn{4}{c|}{ View angle (degrees) } \\
\cline { 2 - 5 } MAE & All & $<\mathbf{2 0}$ & $<\mathbf{1 5}$ & $<\mathbf{1 0}$ \\
Stdev & 43 & 41 & 36 & 26 \\
Cases & 40 & 41 & 35 & 19 \\
\hline
\end{tabular}




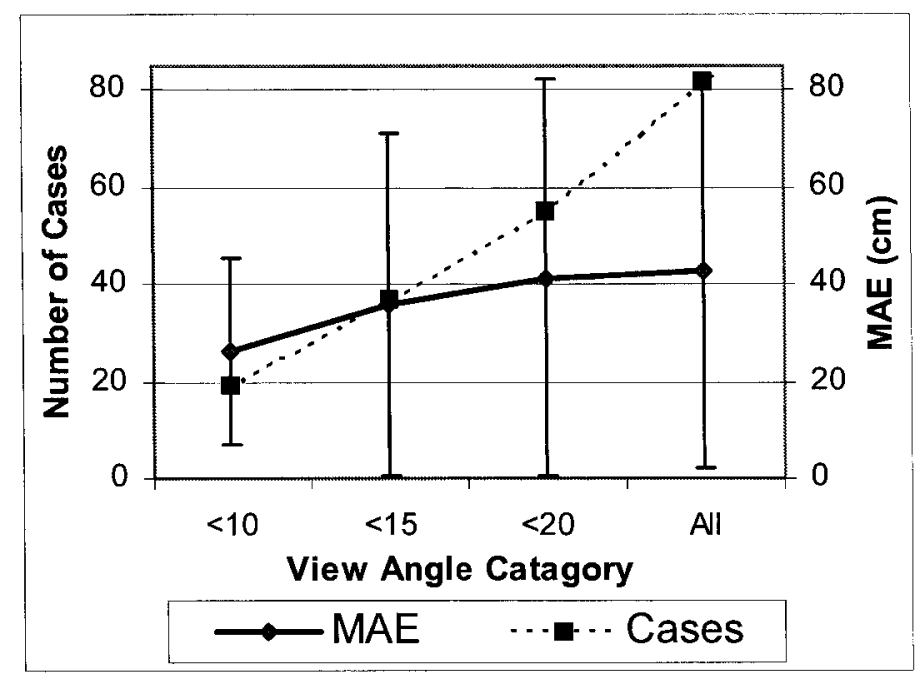

Figure 5.4.7: Error in average crown diameter measured from automated crown delineations. Error bars are \pm 1 standard deviation.

\subsubsection{Discussion}

Detection and delineation accuracy increases with tree size. Trees greater than about 60 $\mathrm{cm}$ in diameter were consistently detected and delineated with good accuracy. However, due to tree clustering and competition effects, some trees up to $1 \mathrm{~m}$ in diameter were either not detected or poorly delineated.

There are numerous factors that affect detection and delineation results. In this dataset, image resolution, image spectral quality, topography, and view angle contributed to the observed detection and delineation errors. These factors are often highly interrelated making it difficult to isolate and quantify their individual effects. The following is a brief discussion of how each of these factors may have contributed to error in this dataset. 
The resolution used in this study was chosen to maximize image coverage and maintain sufficient detail to allow for detection of smaller tree crowns. However, $15 \mathrm{~cm}$ pixels cannot detect very small trees. Typically at least 4-7 pixels are required to detect and delineate a crown. At higher resolutions the greater image detail can improve both detection and delineation as crown boundaries become more distinct. If trees in the 10-40 $\mathrm{cm}$ crown diameter range (very small for the age of this forest) must be consistently detected and delineated, the photos should be scanned at $5 \mathrm{~cm}$ (such a resolution is probably not beyond the limits for this film), with an associated increase in file size.

Spectral quality is also important, as the first step in isolating the tree crowns is to extract all conifer pixels in the imagery. In this dataset bright conifer pixels and bright soil pixels were very similar in all bands, making this separation difficult and prone to errors that propagate through to the final individual tree crown isolation results. This is likely a sensor effect that could be alleviated by using a digital sensor or by setting film exposure to reduce the spectral similarity of bright soil and vegetation.

Topography is likely to have contributed to some error as there are several steeply sloped areas and all blocks are positioned on low to moderate slopes. Topography affects both the spectral properties of the targets being imaged as well as the image resolution. Crowns closer to the sensor will appear larger than those farther away. This is not a significant problem for detection, but does increase errors in crown delineated diameters. Using a suitable (high resolutionlaccuracy) digital elevation model and orthorectifying the imagery would help to reduce errors associated with topography. The spectral effects 
of topography are dependant on the slope aspect and sun direction, as areas sloping away from the sun will be darker than areas sloping towards the sun. Thus, in extracting crown pixels the chosen method must be made flexible enough to extract the brighter and darker pixels for similar objects occurring on different slopes, or a topographic brightness correction algorithm must be implemented.

View angle effects are probably the most significant error sources of all those listed here. Both geometric and spectral (brightness) effects occur. At larger view angles, the sides rather than the tops of trees are imaged causing detection and delineation errors. Large view angles can also cause detection error by hiding trees from the sensor if a smaller tree is located behind a larger one. The spectral differences associated with view angle result from the sun-sensor view angle directions. Energy received by the sensor from the image side closer to the sun is forward scattered from the objects to the sensor, while energy received by the sensor from the image side farther from the sun is backscattered from the objects to the sensor. Backscattering tends to more fully illuminate the tree crown improving detection and delineation, whereas forward scattering at large view angles ( $>$ $15^{\circ}$ ) leaves a significant portion of the crown shaded, resulting in higher detection and delineation error. The results presented above are for all view angles in the imagery. At angles $>30$ degrees from nadir, errors were severe. When only a 10-degree view angle is considered, the RMSE dropped to $20 \%$ for detection and $19 \%$ for delineation. In future work, for full coverage mapping, either a narrow angle sensor should be used, or photography such as that of this study should be acquired with very large overlap (e.g., > 
$80-90 \%)$ so that detection-delineation can be restricted to the centre portions of the images (with an associated increase in number of images to be acquired).

Overall, for image data such as those of this study, accuracy of detection and delineation of individual trees should be considered best within 10 degrees of image centre (nadir), or about $81 \mathrm{~m}$ on the ground and $\sim 3 \mathrm{~cm}$ on the photo. Outside of these areas, tree-by-tree accuracy is reduced but area-based assessment may be acceptable (see Tables 5.4,1 and 5.4.5) due to balancing of errors of omission and commission. 


\title{
5.5 DEVELOPMENT AND EVALUATION OF AN AUTOMATED APPROACH FOR NON-CROP COMPETITION ASSESSMENT USING LEAF-OFF IMAGERY
}

Citation: Pouliot,D.A., D.J. King and D.G. Pitt. (2006). Automated assessment of hardwood and shrub competition in regenerating forests using leaf-off airborne imagery. Remote Sensing of Environment. In press.

\begin{abstract}
Forest regeneration assessment considers the abundance and condition of crop trees as well as the level of competing vegetation. Most remote sensing research has been conducted on conifer crop tree detection and assessment using imagery acquired in deciduous leaf-off conditions. Some research on competition assessment has been conducted using leaf-on imagery, but it is too costly and time consuming to require both leaf-off and leaf-on image acquisition and analysis for complete regeneration assessment. This paper evaluates the potential of manual and automated methods for assessment of woody stem competition using very high-resolution $(2 \mathrm{~cm})$ leaf-off imagery. The intent is to couple the competition-evaluation methods with previously developed leaf-off conifer assessment methods. The manual method consisted of visual interpretation of the amount of competition based on specific spectral and spatial image properties. The automated method combined texture analysis, classification, and line detection. Results show that competition measures extracted using both methods were strongly related to field measurements. Manual extraction outperformed automated extraction, but the best
\end{abstract}


approach may be to combine them to optimize processing time and achieve the highest possible precision, particularly in areas where competition abundance is estimated to be close to a given silvicultural decision threshold.

\subsubsection{Introduction}

Sustainable forestry depends on successful forest re-establishment after disturbance. In the boreal region, regeneration of coniferous species can be difficult due to the effects of competing vegetation, which reduces light, moisture, and nutrients to desired crop trees. It is widely recognized that for timely and effective coniferous forest re-establishment some form of competition control is required (Walstad and Kuch, 1987; Wang, 2000; Wagner et al., 2001). Typical control options include chemical sprays and a few types of manual extraction or cutting of competition. Determining the optimal level and timing of control is a complex and largely subjective process designed to balance the objectives of crop tree success and cost of re-establishment with other ecological considerations such as soil stability and productivity, nutrient availability, temperature regulation, and wildlife habitat concerns. Further, retention of some competing vegetation can be used to control crop tree form for various end use applications such as dimensional or pulp products (pers. comm. Bell, 2004).

The influence of competing vegetation on the establishment and growth of desired crop trees has prompted research in the field of vegetation management in order to quantify and more clearly understand the relations between competitor and crop growth. One facet of this discipline is the parameterization of competition and crop attributes to aid in 
identification of practical methods for evaluating competition effects on crop trees and to provide input to competition control decision making in operational forest management. To date, considerable research has been undertaken to develop field based measurements that relate crop growth to measurable competitor attributes such as visually estimated cover, height, proximity, and light interception (Morris, 1990; Burton, 1993; Comeau, 1993; Ter-Mikaelian et al., 1999; Bell et al., 2000).

Remote sensing research in regeneration assessment has been primarily concerned with assessment of the abundance, spatial distribution, and condition of conifer crop trees using leaf-off imagery (Goba et al., 1982; Hall, 1984; Hall and Aldred, 1992; Gougeon and Leckie, 1999; Brown and Fletcher, 1999; Price and Davison, 1999; Pouliot et al., 2002; Pouliot and King, 2005a; Pouliot et al., 2005). Remote sensing has not been widely researched for competition assessment. Of the few previous studies, leaf-on aerial photography has been used for identification of general cover types (e.g., Pitt and Glover, 1993; Pitt et al., 2000) and high resolution (2.5 cm) digital camera imagery has been assessed for cover and leaf-area estimation (Haddow et al., 2000). Leaf-on conditions provide the best opportunity to assess competition because leaves are clearly visible to the sensor. However, it is difficult, if not impossible, to assess coniferous crop tree attributes in these conditions because the trees are either hidden from the sensor or cannot be easily distinguished from the surrounding competing vegetation. Conversely, leaf-off conditions provide a clear view of coniferous crop trees, but competition is not easily evaluated. This requirement for two sampling periods to satisfy overall objectives of both 
conifer and competition evaluation has hindered the adoption of remote sensing in operational regeneration assessment.

The purpose of this research was to evaluate the potential of high-resolution leaf-off imagery to assess deciduous woody competition abundance. If successful, the methods could be coupled with conifer assessment methods that also use leaf-off imagery (e.g., Pouliot et al., 2002; Pouliot et al., 2005). Both manual interpretation and automated data extraction from imagery were evaluated in modeling field based measures of competition abundance. As this is the first known study of competition assessment using leaf-off imagery, some background on the characteristics of woody competition in highresolution leaf-off imagery is provided to justify the methodological approach that was taken. The approach integrated common automated methods of classification, texture analysis, and morphological feature extraction to establish a base from which longer term research could be developed.

\subsubsection{Methods}

\subsubsection{Study Sites}

The study sites consisted of three operational cutovers northeast of Sioux Lookout, Ontario in the Buchanan Inc. forest management unit. The three cutovers represented various regeneration conditions typical of the region that reflected soil and microclimate conditions. They were labeled High $\left(50^{\circ} 46^{\prime} \mathrm{N}, 91^{\circ} 25^{\prime} \mathrm{W}\right)$, Moderate $\left(50^{\circ} 18^{\prime} \mathrm{N}, 91^{\circ} 39^{\prime} \mathrm{W}\right)$, and Low $\left(50^{\circ} 50^{\prime} \mathrm{N}, 91^{\circ} 21^{\prime} \mathrm{W}\right)$ based on competition intensity. Conditions at these sites were presented in detail in Pouliot et al. (2005) so, for brevity, only the information 
relevant to competing vegetation is presented here. Figure 5.5.1 shows example ground conditions and competition levels at each site.

The High site supported a variety of herbaceous and woody competition species indicative of rich soil with moderate moisture. Woody vegetation was abundant with a mean density of $8500 \mathrm{stems} \mathrm{ha}^{-1}$ for trees above $2 \mathrm{~m}$ in height. This layer was dominated by alder (Alnus spp.), but also contained aspen (Populus tremuloides), white birch (Betula papyrifera), willow (Salix spp.), red-osier dogwood (Cornus stolonifera), and beaked hazel (Corylus cornuta). Grass was the most abundant herbaceous vegetation, occurring in patches where woody competition density was low.

Competition at the Moderate site consisted of pockets of high-density aspen and lower density birch with a mean density of 3400 stems $^{-1} a^{-1}$ for trees above $2 \mathrm{~m}$ in height. Aspen tended to be considerably taller than birch. Herbaceous vegetation was dominated by grass and moss in moist depressions. In higher areas, various low woody shrub types were abundant.

The Low site had little to no competing woody vegetation greater than $2 \mathrm{~m}$ in height. Woody species included alder and willow, while herbaceous competitors included Labrador tea (Ledum groenlandicum), mosses, lichens, and some sparse grasses. For all sites crop trees were less than $2 \mathrm{~m}$ in height. 


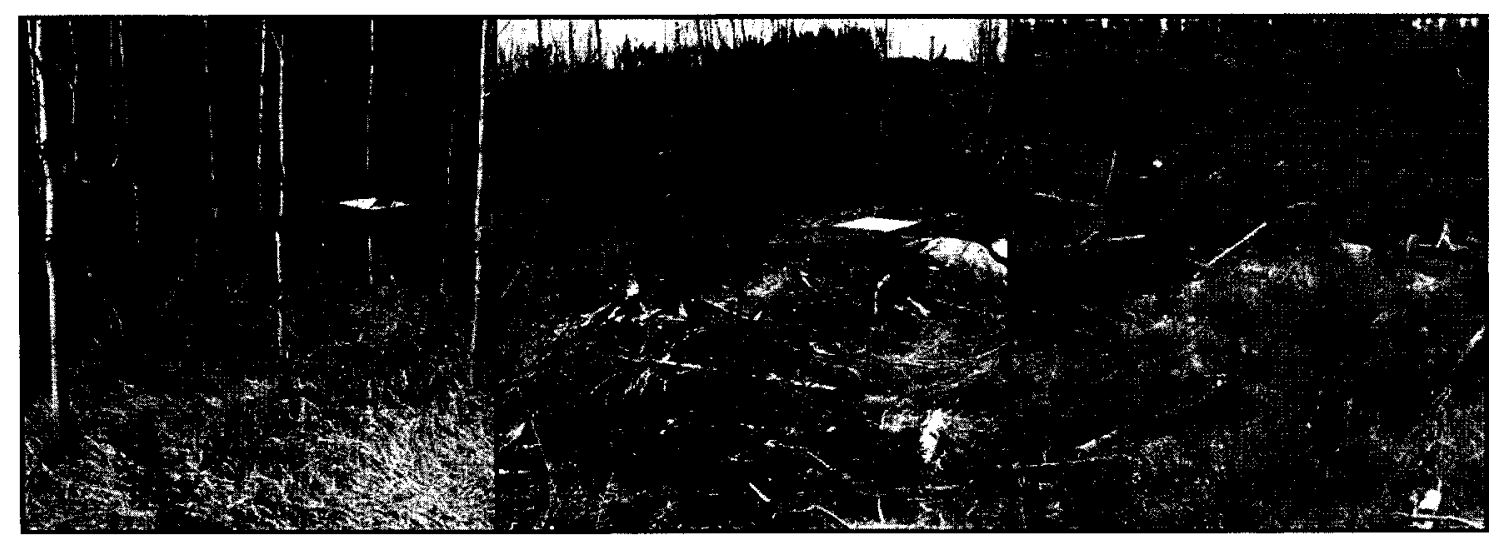

Figure 5.5.1: Example ground conditions at each site. Left - high transect, middle - Moderate transect, right - Low transect.

\subsubsection{Field Data}

Circular sample plots of $3 \mathrm{~m}$ radius were established at $30 \mathrm{~m}$ intervals along a predetermined transect. Their positions were subjectively determined in order to represent the full range of conifer tree species, tree density, crown sizes, and woody competitor abundance at each site. For visual reference in the airborne imagery, plot centers were marked with a $40 \times 40 \mathrm{~cm}$ white board, mounted on a $1.0 \mathrm{~m}$ tall stake. The height of each woody stem in the plot that was taller than $0.5 \mathrm{~m}$ was recorded.

Two field measures of competition abundance were evaluated as dependent variables: stem density and a simple competition index (CI) for three height classes, $>0.5 \mathrm{~m},>1 \mathrm{~m}$, and $>2 \mathrm{~m}$. CI was calculated as the sum of woody competition heights in the plot above the defined height threshold. Stem density is a more intuitive measure of competition abundance and is typically used as the minimum information requirement for competition control treatment decisions. However, CI captures both height and density information. 


\subsubsection{Image Data}

Initially, two sensor types were assessed for this study: multispectral colour infrared digital camera imagery with $6 \mathrm{~cm}$ pixels (as used for conifer assessment in Pouliot et al., 2005 ) and 1:760 scale $70 \mathrm{~mm}$ colour photography scanned to $2 \mathrm{~cm}$ pixels. The digital camera image pixel size was not quite small enough to discern woody competition stems so the photography was selected. It was acquired on May 11, 2002 between 11:30 - 15:00 using a Hasselblad aerial camera with a $100 \mathrm{~mm}$ focal length lens. The camera was mounted in a boom system attached to the undercarriage of a Bell Ranger helicopter and flown at $185 \mathrm{~m}$ above ground. The five-inch prints were scanned at $1000 \mathrm{dpi}$ pixel density using a standard desktop scanner. Plots were extracted as $600 \times 600$ pixel subsets and concatenated into a single image file for processing. In total, 79 plots were extracted; 43 from the High, 21 from the Moderate, and 16 from the Low transects. Different numbers of plots were used due to constraints on the imagery for the analysis requiring imagery only from the backscatter direction (see next section). The High site data contained most of the variance in competition levels, the Moderate site most of the remainder, while the Low site did not contain any detectable competition. The Low site was included to increase the variance of other scene components such as soil, coarse woody debris, and rock, which are often present in operational conditions and which can have similar spectral and spatial image characteristics to woody competition.

\subsubsection{Distinctive Image Properties Linked to Competition Abundance}

Examination of the photos revealed several potentially useful image properties of competing shrub and hardwood vegetation (Figure 5.5.2). Spectral properties of 
branches, stems, and reproductive structures were often distinct, but in some places they were similar to other cover types. For example, hardwood competitors such as poplar and older birch generally had grey-white stems and branches that appeared spectrally similar to rock and snow, which were present in localized patches. Fortunately, their spatial structures were quite different with their straight stems being more visible with increasing view angle in the backscattering direction (Figure 5.5.2A). Potential was evident for both image texture and linear feature (stem) extraction and analysis. In the forward scattering region of the image, hardwood stems were not clearly visible. Hardwood shadow presence, size and form (Figure 5.5.2B) were also initially considered as possible characteristics for assessment of competition type and abundance. Shadows were more distinctive in the forward scattering direction if the background was relatively uniform. However, shadows cast on dark ground were difficult to detect and shadows cast by proximal objects such as a conifer crown and a hardwood stem can overlap (as shown in Figure 5.5.2C). These were common occurrences in the imagery so shadow analysis was not considered further.

Shrub competition such as alder, willow, and hazel generally had brownish woody structures (Figure 5.5.2D) that appeared spectrally similar to soil cover (Figure 5.5.2E), especially for wetter soils. Shrubs did not have specific structural properties associated with sun-sensor geometry as did hardwoods, but they did appear to have some potentially useful texture properties that were distinctive from soil. 


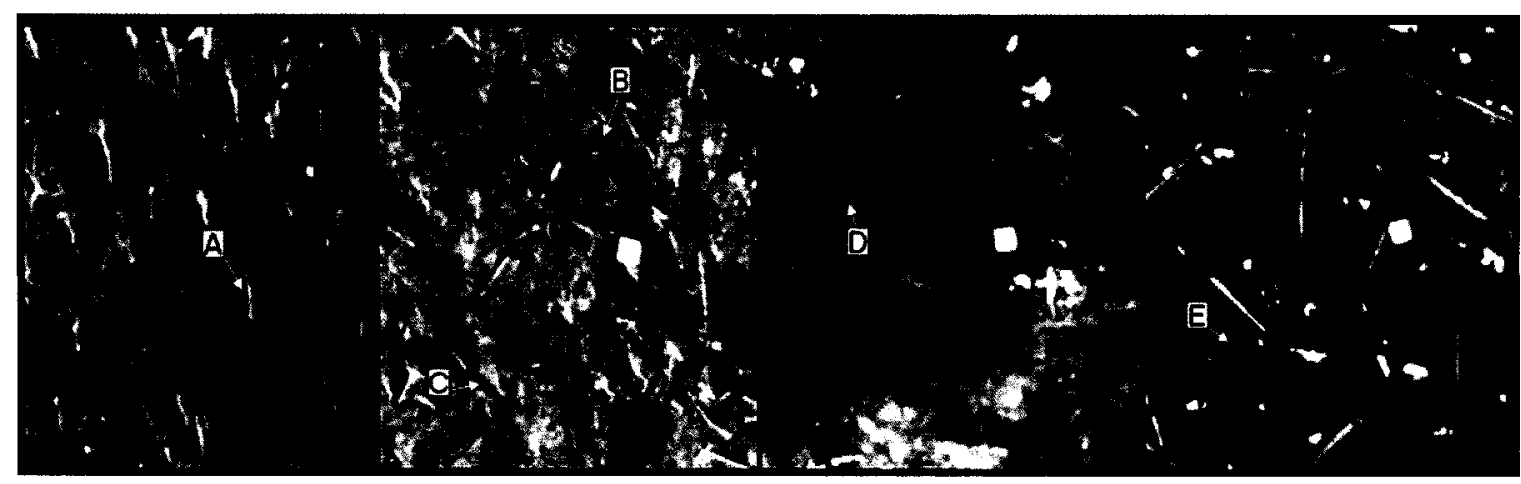

Figure 5.5.2. Characteristics of competition in leaf-off imagery. A) hardwood stems in the backscatter portion of the image, B) clearly visible hardwood stem shadows, C) obstructed hardwood stem shadow, D) shrub competition, E) soil that can appear spectrally similar to shrub competition.

Based on these visually distinctive hardwood and shrub properties, spectral, textural and morphological analyses were conducted and integrated into the competition assessment methodology described below.

\subsubsection{Extraction of Hardwood and Shrub Competition Abundance Measures}

Measures of competition were extracted from the imagery manually using visual interpretation and with an automated algorithm. These measures were then used to develop models with the field measurements of stem density and CI.

The imagery used for hardwood stem extraction was restricted to view angles greater than $4^{\circ}$ in the backscatter direction as stems of poplar were most distinct in this region. The manual and automated analyses were designed to estimate the number of stems, average stem length, and total number of stem pixels within the image plots. Stem length was standardized to remove distortions due to relief displacement (Equation 5.5.1) within the given image view angles $\left(4^{\circ}\right.$ to $\left.17^{\circ}\right)$. 
$h=\frac{d H}{r}$

where $h$ is the true object height, $H$ is the flying height, $d$ is the measured displacement of the object in the image, and $r$ is the distance from nadir to the top of the image object (Lillesand and Kiefer, 2000).

View and scattering angle images, following the methodology given in Pellikka et al. (2000), were produced for each image and the mean values for both were extracted for each plot. This information was used to evaluate whether these variables were significant in multiple regression competition models by accounting for sun-sensor geometry effects.

For shrub competition, total cover (number of pixels with shrub presence) was extracted as well as a fuzzy measure of total cover. The purpose of the fuzzy measure was to make the cover estimates more robust by providing a graded estimate of cover instead of a hard classification of shrublno-shrub for each pixel. The fuzzy cover $(F C)$ estimate was based on Euclidean distance calculated as:

$$
F C=\frac{1}{1 \times 10^{5}} \sum_{i=1}^{n}\left[442-\sqrt{\sum_{j=1}^{m}\left(x_{i j}-\bar{x}_{j}\right)^{2}}\right]
$$

where $i$ is a shrub cover pixel, $n$ is the number of shrub pixels in the plot, $x_{i j}$ is the pixel value for band $j, \bar{x}_{j}$ is the mean value for the band $j$ computed from the training sample pixels, and $m$ is the number of bands. The constant 442 represents the maximum 
Euclidean distance value attainable with 3-band 8-bit data and is used to reverse the scale of the data such that pixel values closer to the mean represent larger values than those further away. Thus, as $F C$ increases shrub competition also increases.

\subsection{Manual Interpretation of Competition Properties}

Manual interpretation was accomplished by first training the interpreter (first author) on a subset of image data containing ground truth to recognize the properties of hardwood and shrub competition. This was undertaken as part of an initial exploratory analysis to identify potentially useful image features unique to shrub and hardwood competition as described above. Field measurements and pictures taken of the plots from different perspectives on the ground were used to aid training. Twelve plots were used for training, 5 plots from the High transect, 4 from the Moderate transect, and 3 from the Low transect. For hardwood competition, a line representing the stem was manually digitized. For shrub competition, identified areas were digitized as polygons. Both were conducted independently of the automated algorithm processing described below.

\subsection{Automated Extraction of Competition Image Properties}

Automated image analysis methods were explicitly selected to capitalize on the spectral, spatial and structural image properties of competing vegetation as discussed above. 


\section{Classification and Texture Analysis}

Supervised maximum likelihood classification was applied using nine classes: shrub, hardwood, shadow, snow-rock, conifer, grass, light soil, dark soil, and red colored soil. For each class, six plots were selected and three samples were taken within each plot. Plots and the samples they contained were then assigned randomly as either training or validation data. Thus, for each class nine samples were used for training and nine for validation. Separate classifications were conducted for hardwood and shrub to optimize the spectral and textural features used for each of these classes.

Co-occurrence texture measures were used, as they are commonly applied for extraction of forest information at a variety of scales (Wulder et al., 1996; Peddle and Franklin, 1991; Franklin et al., 2000; Haddow et al., 2000; Treitz and Howarth, 2000; Tuominen and Pekkarinen, 2005) and have the potential to discriminate between a large number of determinate and statistical patterns. They are based on the co-occurrence matrix, which tabulates the probability of pairs of pixel gray-levels within a sample window. Several measures of texture can be derived from the matrix (e.g., Haralick et al., 1973). For a given texture measure there are several parameters that must be considered: 1) sample window size, 2) inter-pixel sample distance, 3) inter-pixel sample direction, and 4) quantization level. Selection of the most discriminating texture measure and the best parameter set for a given texture measure is not straightforward and typically involves trial and error or an automated computer intensive approach. As this was the first study of its type, the computer intensive approach was used. Texture measures included Homogeneity (HOM), Contrast (CON), Mean (MN), Variance (VAR), Entropy (ENT), 
Dissimilarity (DIS), and Angular Second Moment (ASM). Window size was varied from 5 to 47 pixels in a 7-pixel step interval. The upper limit was selected as the largest size that could be used with minimal class boundary crossings. The spatial variability of each class was high with many small patches on the order of one to a few meters in size. Thus, we felt that a window size of 47 pixels $(94 \mathrm{~cm} \times 94 \mathrm{~cm})$ was the largest that should be considered. It also represented a reasonable upper limit for processing time, which increased exponentially with window size $(\sim 20$ hours for the $47 \times 47$ window and the $7300 \times 4300$ image database). Inter-pixel sample distances of 1,3 , and 5 pixels were tested. Directionally invariant sampling of pixel pairs was used because no distinct directional properties were visible in the imagery. Quantization was held constant at 6 bits (64 gray levels). These texture measures are subsequently referred to by their 'acronym_window size_pixel spacing' as in the following example: HOM_5_1 (Homogeneity with $5 \times 5$ window size and sample distance of 1 pixel). CON and VAR were non-normally distributed so a log transformation was applied.

The output image for each texture measure was included with the three original spectral bands to calculate the Jeffries-Matsushita (JM) separability measure (Richards, 1993) for the hardwood and shrub classes as shown in Equation 5.5.3.

$$
J M_{i j}=2 \times\left(1-e^{-a_{i j}}\right)
$$

where

$a_{i j}=0.125 \times\left(M_{i}-M_{j}\right)^{T} \times\left(C_{a}\right)^{-1} \times\left(M_{i}-M_{j}\right)+0.5 \times \ln \left(\left|C_{a}\right| \div\left(\left|C_{i}\right| \times\left|C_{j}\right|\right)^{0.5}\right)$

$M_{i}, M_{j}=$ mean vectors for class i or j;

$C_{i}, C_{j}=$ Covariance matrix for class $\mathrm{i}$ or $\mathrm{j}$; 
$C_{a}=\left(C_{i}+C_{j}\right) / 2$

The minimum separability between the hardwood or shrub class and one of the other classes was used to compare the classification potential of the different texture measures. A simple leave-out resampling approach was used to reduce the dependence of the separability measure on training data. For each iteration, seven of the nine samples for each class were randomly selected and used to calculate separability. The mean and lower $95 \%$ bound of the J-M distance for 30 iterations were calculated and used to compare the texture measures.

Because of the high correlation between some co-occurrence texture measures (HallBeyer, 2004), they were grouped to minimize between group correlations. The best measure from each group was used in classification in order maximize the amount of independent information in the input variables. Three groups were identified based on Hall-Beyer (2004): 1. Contrast - HOM, CON, VAR, DIS; 2. Orderliness - ENT, ASM; 3. Descriptive - MN.

Finally, as very high-resolution imagery can be strongly influenced by local noise, a Gaussian noise reduction filter with standard deviation $(\sigma)=1$ was evaluated for potential classification improvement.

To evaluate hardwood or shrub class accuracy in each of the classifications, the conditional kappa coefficient was used. 
$K U_{i}=\frac{N\left(x_{i i}\right)-\left(x_{i+} \times x_{+i}\right)}{N\left(x_{i+}\right)-\left(x_{i+} \times x_{+i}\right)}$

Where $K U_{i}$ is the user's kappa coefficient for class $i, N$ is the total number of sample pixels, $x_{i i}$ is the number of correctly classified pixels, $x_{i+}$ equals $x_{i i}$ plus commission error, and $x_{+i}$ equals $x_{i i}$ plus omission error. For producer's accuracy $\left(K P_{i}\right)$ the denominator in Equation 5.5 .5 becomes $N\left(x_{+i}\right)-\left(x_{i+} \times x_{+i}\right)$. To obtain a measure of class accuracy that accounts for both omission and commission error, the average of $K U_{i}$ and $K P_{i}$ was taken $\left(K A_{i}\right)$.

\section{$\underline{\text { Line Detection }}$}

Line detection of hardwood stems was accomplished using a slightly modified version of Steger's (1998) method. This involved determination of the optimal scale, identification of candidate line points, and linking points into lines. Modifications to Steger's criteria included how candidate line points were selected and linked to create lines.

Optimal detection for a given line width is dependent on image scale. For line detection, the relation between the minimum smoothing level and line width is $\sigma \geq w / \sqrt{3}$, where $w$ is the line width and $\sigma$ is the standard deviation parameter used in Gaussian filtering (Steger, 1998). In this study, hardwood stems were generally about 3 pixels wide. Thus, $\sigma=2$ was selected for Gaussian smoothing before implementation of the line detection algorithm. 
The radiometric topography of linear features in an image consists of narrow valleys if they are dark or ridges if they are bright. In one dimension, the location of a ridge is where grey-level values perpendicular to the line have a first derivative value of zero and local minima in the second derivative. To detect lines in two-dimensions the derivatives in the horizontal, vertical, and diagonal directions can be found for the image $f(x, y)$ using

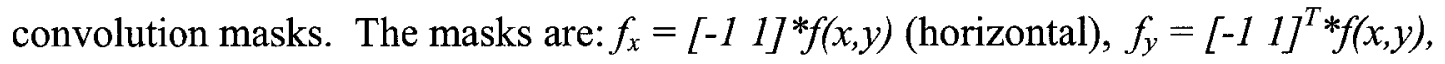
(vertical), and $f_{x y}=\left[\begin{array}{lll}-1 & 1\end{array}\right]^{T} * f_{x}$ (diagonal), where $\mathrm{T}$ indicates the transpose. The second derivatives in the $\mathrm{x}$ and $\mathrm{y}$ directions, $f_{x x}$ and $f_{y y}$, are obtained by convolving the first derivative responses with these same masks. These values define the Hessian matrix as:

$H(x, y)=\left[\begin{array}{ll}f_{x x} & f_{x y} \\ f_{x y} & f_{y y}\end{array}\right]$

where the maximum absolute eigenvalue of the Hessian matrix gives the line strength and the associated eigenvector gives the perpendicular direction to the line as a unit vector $\left(n_{x}, n_{y}\right)$. The sub-pixel position of the line can then be calculated as:

$$
t=\frac{f_{x} n_{x}+f_{y} n_{y}}{f_{x x} n_{x}{ }^{2}+2 f_{x y} n_{x} n_{y}+f y_{y} n_{y}{ }^{2}}
$$

A point is considered a line if $\left(t n_{x}, t n_{y}\right) \in[-1 / 2,1 / 2] \times[-1 / 2,1 / 2]$. In this implementation, a further constraint for acceptance of line points was applied to ensure that the line direction had an orientation similar to that of the imaginary line joining the pixel and nadir (the pixel azimuth), as stems should appear to be leaning away from nadir in this 
direction due to view anglelrelief displacement. A tolerance of $10^{\circ}$ was used for this criterion based on visual assessment of several threshold tests.

As in Steger (1998), to link detected line points, local maxima positions were identified with a $3 \times 3$ local maximum filter and used as starting points for line following. For each, line following was performed by moving to the next pixel in a $3 \times 3$ window that maximized the line strength $\left(l_{s}\right)$ and minimized the angular difference between lines $\left(l_{a}\right)$. Line strength and angular difference were scaled to a range of $0-1$ by dividing by their maximum respective values in the image. For angular difference, the scale was reversed by subtracting the maximum angular difference such that larger angular differences took on small values of $l_{a}$ and small differences took on large values. Line strength and angular difference were summed as $l_{s}+0.5 l_{a}$. In this implementation, line strength was considered to be more important for line following than the distance between candidate line points as used in Steger's implementation because thresholding on line strength was not used. Instead, the final lines were extracted as the intersection of line points with the hardwood classification results. Further, small lines less than 5 pixels long were removed.

\subsubsection{Modeling Hardwood and Shrub Competition Abundance}

Correlation analysis and stepwise least squares regression were used in initial exploratory analysis to determine meaningful variables for competition modeling. However, the use of least squares regression can lead to biased estimates of regression parameters if errors in the independent variable are large compared to that of the dependent variable (Draper and Smith, 1998). To account for this in final model parameter estimates, geometric mean 
regression was used, which is a robust estimator in this situation (Draper and Smith, 1998). For hardwoods, models were explored for the following variables: average stem length/plot, number of stems/plot, total stem length/plot, mean plot view angle, and mean plot scattering angle. In addition, average stem length/plot and total stem length/plot (STSL) were standardized by view angle and evaluated. For shrubs, total shrub pixel count/plot, a fuzzy pixel count/plot (FZCN), mean plot view angle and mean plot scattering angle were evaluated.

It was also desirable to develop a model that could be used to predict the level of both shrub and hardwood competition combined (hardwood + shrub). This was accomplished by combining the individual shrub and hardwood models and simplifying (i.e. if $s h r u b=$ $m s F Z C N+b s$ and hardwood $=m h S T S L+b h$, then hardwood $+s h r u b=$ $m s F Z C N+b s+m h S T S L+b h)$. It is appropriate in this case because these variables were only correlated with their associated dependent variable. All other combinations of correlations between dependent and independent variables for these two models were low $(\mathrm{r}<0.2)$

To evaluate model robustness and predictive ability, an iterative resampling procedure was applied. Resampling analysis with a 1/3 data leave-out-rule was used with 1000 iterations. The means and standard deviations were obtained for the model parameters (slope and offset), modeling efficiency statistic $\left(E F=1-\sum\left(y_{i}-\hat{y}\right)^{2} / \sum\left(y_{i}-\bar{y}_{i}\right)^{2}\right.$ for the line $y=\hat{y}$; Mayer and Butler, 1993), model bias $\left(B I A S=\left[\sum y_{i}-\hat{y}_{i}\right] / n\right)$, and mean absolute error 
$\left(M A E=\left[\sum\left|y_{i}-\hat{y}_{i}\right|\right] / n\right)$. The average of each of these goodness of fit statistics is reported for the $1 / 3$ validation sample from the 1000 iterations.

\subsubsection{Results}

\subsubsection{Extraction of Hardwood and Shrub Abundance Measures}

\subsection{Evaluation of Texture for Competition Classification}

The results of the texture analysis showed that the minimum separabilities of both the hardwood and shrub competition classes were low (Figure 5.5.3). For hardwoods, the minimum separability was with the snowłrock class. Window size had the most substantial effect followed by the type of texture measure. Increasing the sample window size decreased the separability. Most of the texture measures produced similar results, however, the MN measure was least affected by window size and ASM produced considerably lower separabilities. ASM actually decreased the separability compared to that for the original RGB bands alone. No trend was apparent for sample distance. The best texture measures for each of the defined groups for hardwood discrimination were:

1. Contrast-VAR_11_5; 2. Orderliness - ENT_11_3; and 3. Descriptive - MN_23_1. These texture measures are indicated by the arrows in Figure 5.5.3.

For shrubs, separabilities with other classes were lower than for hardwoods due to a strong spectral similarity with the dark soil class. Window size had the most influence on separability, but in this case, increasing it resulted in increased separability. As with the hardwood results, ASM produced the lowest separability, but it was greater than that of 
the original RGB bands alone $(\mathrm{J}-\mathrm{M}=0.84)$. No trend for sample distance was identified. The best texture measures for each of the defined groups were: 1. Contrast - DIS_47_3; 2. Orderliness - ENT_47_1; and 3. Descriptive - MN_35_1.

Both the hardwood and shrub separability results show that for some texture measures and input parameters, the difference between the mean and lower bound J-M distance varied amongst re-sampled training data iterations, indicating a dependence on the training data used. For example, ASM shows the largest difference for both the shrub and hardwood classes and thus appears to have been most affected by the training data used.

\subsection{Classification Results}

Table 5.5.1 shows the classification accuracies for hardwoods and shrubs with different combinations of spectral and textural features. The hardwood class produced a moderate separability, but had low average kappa $(K A)$ for almost all feature combinations tested. The shrub class was the opposite, with low separability and higher $K A$ values compared to the hardwood class. This discrepancy resulted from the information utilized in the separability and $K A$ measures, as separability only considered confusion between two classes at a time, whereas $K A$ is a summary measure of confusion for the given class with all other classes. Gaussian smoothing and texture incorporation produced the best results for each class. For hardwoods, the optimal set of input features included all Gaussian filtered spectral bands and all the selected texture measures. A similar feature combination was found to be optimal for the shrub class, except that only the two best 
texture measures were included. These optimal feature combinations are bolded in Table 5.5.1. 

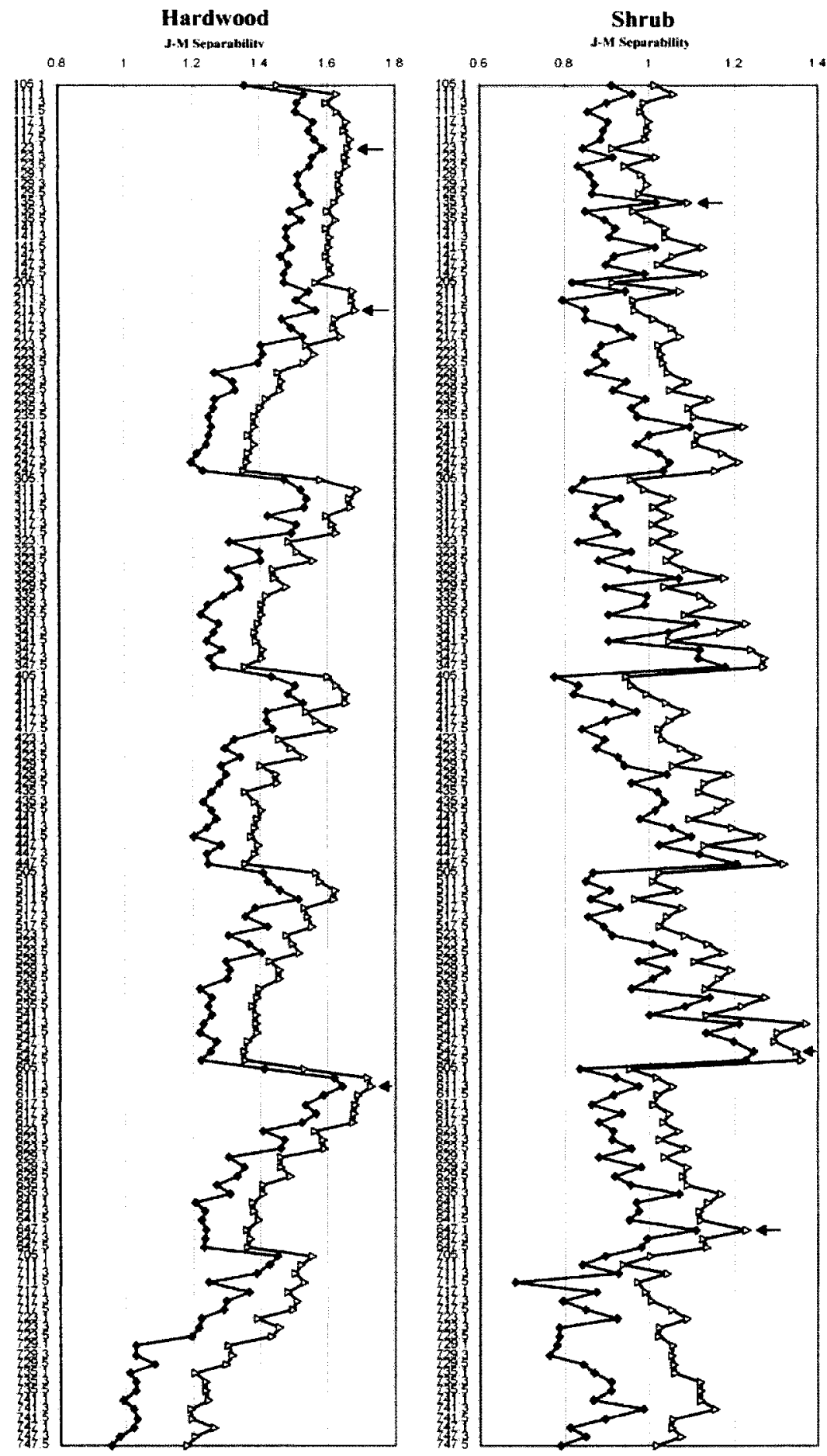

Figure 5.5.3: Hardwood and shrub class separability results for the original RGB bands with an additional texture measure. Values are the mean (triangles) and lower $95 \%$ confidence bound ( $-2 \mathrm{~s}$, diamonds) obtained from 30 iterations of resampled training data. For display purposes the graphs have been rotated, the $\mathrm{x}$-axis is the J-M separability measure and the $\mathrm{y}$-axis is the texture measure label. The first digit in the label identifies the method (1-MN, 2-VAR, 3-HOM, 4-CON, 5-DIS, 6-ENT, 7-ASM). The following two digits give the window size and the decimal number is pixel sample distance. Arrows identify the texture measure that produced the highest minimum separability for each of the defined texture measure groups. 
Table 5.5.1: Classification accuracy for the selected feature combinations used in classification. Commission and omission errors in training and test columns are in numbers of pixels.

\begin{tabular}{|c|c|c|c|c|c|c|c|c|}
\hline & Data Features & $\begin{array}{l}\text { Total } \\
\text { Samples }\end{array}$ & Correct & $\begin{array}{c}\text { Commission } \\
\text { Error }\end{array}$ & $\begin{array}{c}\text { Omission } \\
\text { Error }\end{array}$ & $\begin{array}{c}\text { User's } \\
\text { Kappa } K U_{i}\end{array}$ & $\begin{array}{l}\text { Producer's } \\
{\text { Kappa } K P_{i}}\end{array}$ & $\begin{array}{c}\text { Average } \\
\text { Kappa } K A\end{array}$ \\
\hline \multirow{8}{*}{ 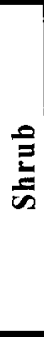 } & RGB & 33637 & 9407 & 726 & 2465 & 0.889 & 0.703 & 0.796 \\
\hline & RGB_GF & 33637 & 10464 & 245 & 1408 & 0.965 & 0.826 & 0.895 \\
\hline & RGB+S1 & 33637 & 8756 & 609 & 3116 & 0.899 & 0.636 & 0.768 \\
\hline & RGB $+S 2$ & 33637 & 9084 & 642 & 2788 & 0.898 & 0.670 & 0.784 \\
\hline & RGB+S3 & 33637 & 9777 & 350 & 2095 & 0.947 & 0.748 & 0.847 \\
\hline & RGB_GF+S1 & 33637 & 9541 & 237 & 2331 & 0.963 & 0.723 & 0.843 \\
\hline & RGB_GF+S1,S2 & 33637 & 10603 & 185 & 1269 & 0.973 & 0.843 & 0.908 \\
\hline & RGB_GF+S1,S2,S3 & 33637 & 10394 & 177 & 1478 & 0.974 & 0.818 & 0.896 \\
\hline \multirow{8}{*}{ 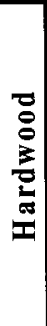 } & RGB & 33637 & 866 & 806 & 62 & 0.504 & 0.930 & 0.717 \\
\hline & RGB_GF & 33637 & 887 & 765 & 41 & 0.524 & 0.954 & 0.739 \\
\hline & RGB+H1 & 33637 & 888 & 1025 & 40 & 0.449 & 0.954 & 0.702 \\
\hline & RGB+H2 & 33637 & 847 & 1161 & 81 & 0.405 & 0.907 & 0.656 \\
\hline & RGB+H3 & 33637 & 895 & 987 & 33 & 0.461 & 0.962 & 0.712 \\
\hline & RGB_GF+H1 & 33637 & 900 & 881 & 28 & 0.491 & 0.968 & 0.730 \\
\hline & RGB_GF+H1,H2 & 33637 & 898 & 1127 & 30 & 0.428 & 0.966 & 0.697 \\
\hline & RGB_GF+H1,H2,H3 & 33637 & 896 & 303 & 32 & 0.740 & 0.964 & 0.852 \\
\hline
\end{tabular}

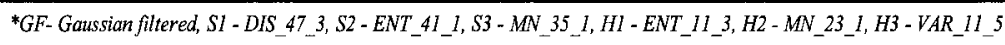

\subsubsection{Modeling Hardwood and Shrub Competition Abundance}

In bivariate modeling of hardwood competition, STSL proved to be the best predictor using either manual or automated extraction. For shrub competition, total pixel count and FZCN were found to provide similar predictive ability. However, FZCN provided a slight improvement and is theoretically more resilient to classification errors than the hard pixel count, so it was retained for further modeling. The mean plot view angle and scattering variables were not found to be significant in any of the multiple regression models evaluated. This suggests that sun-sensor geometry effects were not as significant as other sources of dependent variable variance or that these effects were more complex than could be captured by a simple weighted sum of variable combinations. 


\subsection{Comparison of Competition Measures Extracted Using Manual and Automated}

\section{Methods}

For hardwood competition, Figure 5.5.4 is an example of the results of manual and automated line (stem) extraction for two plots. Overall, most of the same features were identified except several additional small lines were found by the automated algorithm and some lines were not fully connected.

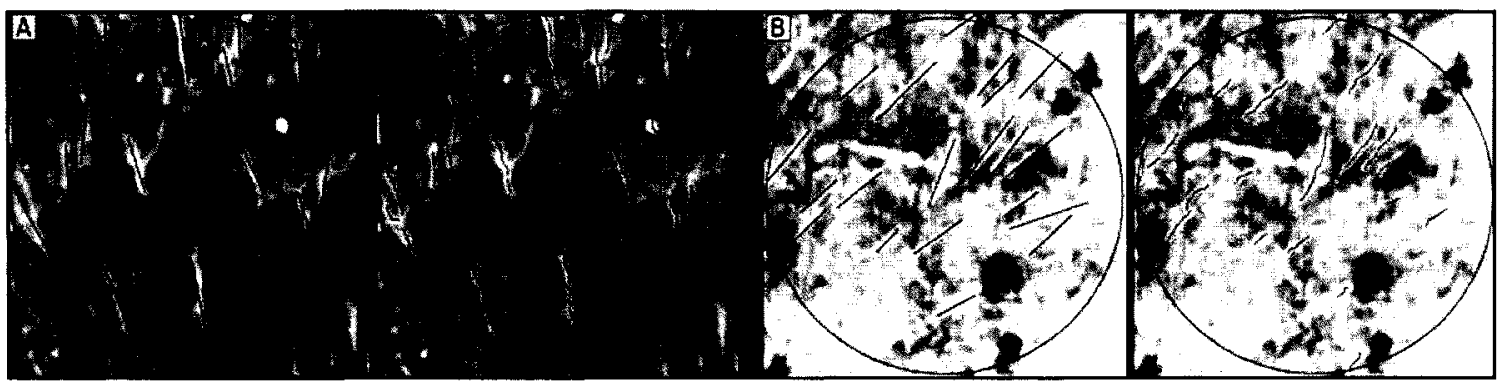

Figure 5.5.4: Example results of hardwood stem detection. In each example the visually interpreted results are on the left and colored blue while the automated results are on the right and colored red.

Figure 5.5.5 shows a scatterplot of STSL extracted using the manual and automated methods. The two measures were strongly correlated with observations equally distributed on either side of the idealized $y=\hat{y}$ line. Woody debris and other small linear features oriented in the same direction as that expected for hardwood stems were the main factors causing overestimation of STSL by the automated method. In some plots where there were no competing stems, the automated method made several commission errors leading to a large range in $\mathrm{x}$ for $\mathrm{y}=0$, while the manual method was not subject to this error. At the high end of the data range (approximately $60-100 \mathrm{~m}$ ), both the manual and automated methods were substantially more variable. 


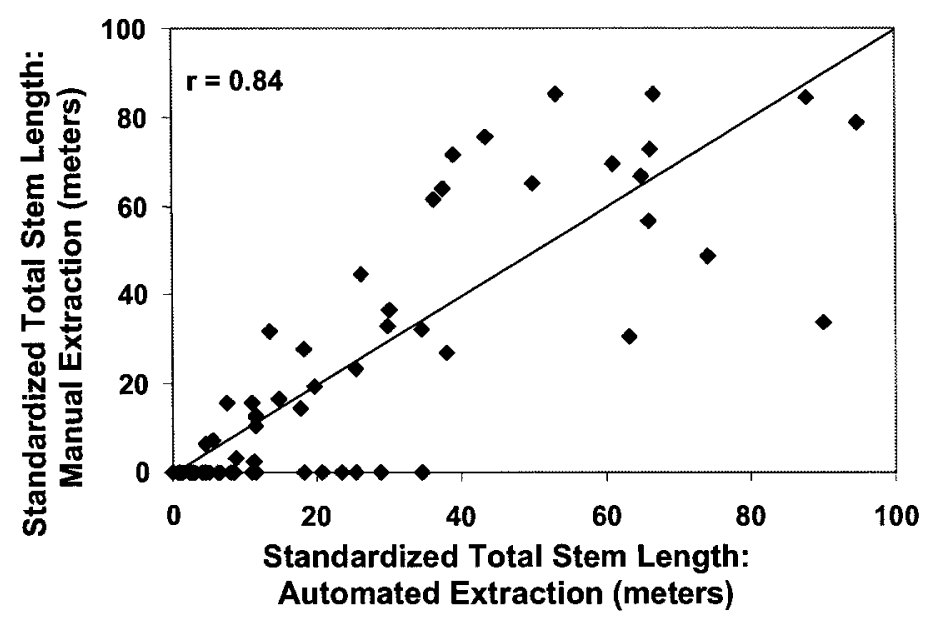

Figure 5.5.5: Scatterplot of manual and automated standardized total stem length of hardwood stems per plot. The line depicts the relation $\mathrm{y}=\hat{\mathrm{y}}$.

For shrub competition, Figure 5.5.6 shows a scatterplot of FZCN extracted using manual interpretation and the automated procedures for each plot. The two measurements are strongly correlated, but the automated estimates are greater at the lower end of the data range and the manual results tend to be larger for larger values. As seen for hardwoods, there are many plots for which the automated method detected shrub competition presence while the manual method did not (i.e. the large range of $x$ values for $y=0$ ). This was due to confusion between shrub and dark soil in the classification. 


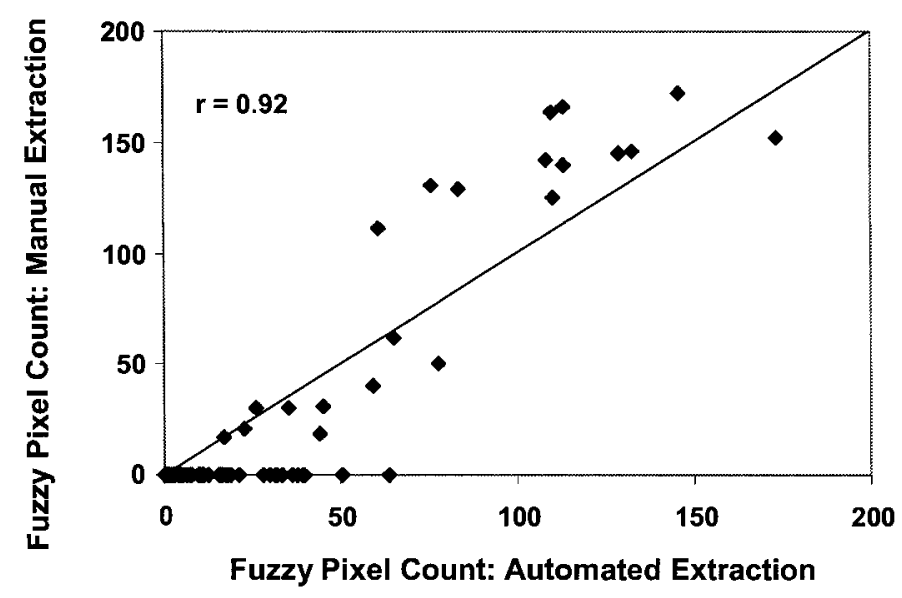

Figure 5.5.6: Scatterplot of manual and automated fuzzy pixel counts per plot for the shrub class. The line depicts the relation $y=\hat{y}$.

\subsection{Competition Index (CI) Modeling}

Table 5.5.2 shows the manual and automated modeling results for the shrub, hardwood, and combined shrub-hardwood competition indices. The model fit statistics are mean values for the 1000 iterations that were implemented using varied random samples. The standard deviations of these statistics are also given in italics. Manual extraction produced the best model fit for all competition response variables with $\mathrm{EF}>0.70$ for all models. Goodness of fit statistics improved when competition height thresholds were used, showing that the competition signal in the imagery was stronger for larger trees, as expected. Hardwood competition was slightly better predicted than shrub competition, having a higher $\mathrm{EF}$ for the $0.5 \mathrm{~m}$ and $2 \mathrm{~m}$ height thresholds.

Automated extraction produced lower goodness of fit statistics and higher standard deviations for estimated parameters. Automated model EFs ranged from 0.57 for shrub competition above $2 \mathrm{~m}$ in height to 0.70 for shrub-hardwood competition above $1 \mathrm{~m}$. 
Hardwood competition was better modeled than shrub competition and model fits improved with increasing height threshold. However, for shrubs and hardwood+shrub, at the $2 \mathrm{~m}$ threshold, reduced model fits were observed, as this height threshold was close to the mean height of shrub competition in the plots, considerably increasing the number of zero cases for the dependent (i.e. field) variable. This caused greater variability where observed values were zero and predicted values were greater than 0 increasing the overall model bias. This effect was not observed for the hardwood competition modeling because hardwoods were typically taller than the $2 \mathrm{~m}$ height threshold. The coefficients from the hardwood+shrub model are not included in the table because they are the same as that given for the bivariate models. 
Table 5.5.2: Model results for the competition index (CI) measured in meters.

\begin{tabular}{|c|c|c|c|c|c|c|c|c|c|}
\hline \multicolumn{4}{|c|}{ Dependent Variable } & \multirow{2}{*}{$\begin{array}{c}\text { Independent } \\
\text { Variable(s) }\end{array}$} & \multicolumn{2}{|c|}{ Model Parameters } & \multicolumn{3}{|c|}{ Model Fit Statistics } \\
\hline & Mean & Stdv & Max & & Slope & Offset & EF & BIAS & MAE \\
\hline \multirow{2}{*}{ CI_H_0.5 } & \multirow{2}{*}{45} & \multirow{2}{*}{59} & \multirow{2}{*}{229} & \multirow{2}{*}{ STSL_MAN } & 2.270 & 3.721 & 0.793 & 0.022 & 18.002 \\
\hline & & & & & 0.161 & 1.994 & 0.071 & 5.908 & 2.345 \\
\hline \multirow{2}{*}{ CI_H_1 } & \multirow{2}{*}{45} & \multirow{2}{*}{59} & \multirow{2}{*}{222} & \multirow{2}{*}{ STSL_MAN } & 2.239 & 3.384 & 0.801 & 0.026 & 17.533 \\
\hline & & & & & 0.155 & 1.853 & 0.078 & 5.890 & 2.358 \\
\hline \multirow{2}{*}{ CI_H_2 } & \multirow{2}{*}{38} & \multirow{2}{*}{55} & \multirow{2}{*}{190} & \multirow{2}{*}{ STSL_MAN } & 2.039 & 0.859 & 0.862 & 0.002 & 12.600 \\
\hline & & & & & 0.135 & 1.341 & 0.043 & 4.486 & 1.824 \\
\hline \multirow{2}{*}{ CI_H_0.5 } & \multirow{2}{*}{46} & \multirow{2}{*}{60} & \multirow{2}{*}{229} & \multirow{2}{*}{ STSL_AUT } & 2.326 & -3.226 & 0.660 & 0.590 & 21.350 \\
\hline & & & & & 0.234 & 2.251 & 0.133 & 7.775 & 3.038 \\
\hline \multirow{2}{*}{ CI_H_1 } & 45 & 59 & 222 & STSI & 2.283 & -3.472 & 0.670 & 0.247 & 21.050 \\
\hline & 45 & 39 & 222 & S1SL_AU1 & 0.228 & 2.141 & 0.140 & 7.557 & 2.911 \\
\hline $\mathrm{CI} \mathrm{H}_{2}$ & 39 & 56 & 190 & STSL & 2.079 & -5.343 & 0.677 & 0.138 & 19.218 \\
\hline & & & & & 0.214 & 1.847 & 0.130 & 7.069 & 2.724 \\
\hline $\mathrm{CI} S 0.5$ & 45 & 64 & 262 & FZCN MAN & 1.359 & 10.872 & 0.720 & $\mathbf{0 . 3 7 3}$ & 20.802 \\
\hline C1_s_0.0 & & & & & 0.129 & 2.541 & 0.140 & 8.067 & 3.618 \\
\hline CI S 1 & 33 & 62 & 247 & F7CN MAN & 1.176 & 1.713 & 0.836 & 0.050 & 13.415 \\
\hline C1_S_1 & & & & & 0.118 & 1.466 & 0.083 & 5.590 & 2.539 \\
\hline $\mathrm{CI} S 2$ & 22 & 52 & 220 & FZCN MAN & 0.950 & -2.966 & 0.787 & -0.461 & 12.152 \\
\hline & & & & & 0.132 & 1.367 & 0.105 & 5.214 & 2.100 \\
\hline CI S 05 & 47 & 65 & 262 & FZCN AUT & 0.152 & -3.424 & 0.586 & -0.208 & 27.046 \\
\hline & & & & & 0.019 & 3.200 & 0.182 & 8.873 & 3.531 \\
\hline CI S 1 & 33 & 62 & 247 & FZCN AUT & 0.132 & -10.745 & 0.677 & -0.241 & 22.442 \\
\hline C_S_1 & 25 & 02 & 241 & |FLCN_AUI & 0.019 & 2.907 & 0.107 & 7.619 & 2.628 \\
\hline $\mathrm{CI} S 2$ & 22 & 53 & 220 & FZCN AUT & 0.108 & -12.937 & 0.573 & -0.301 & 21.224 \\
\hline 1_s_2 & 22 & & & & 0.019 & 2.984 & 0.176 & 7.395 & 2.431 \\
\hline $\mathrm{CI} \mathrm{S}+\mathrm{H} \quad 0.5$ & 91 & 91 & 316 & FZCN_MAN, & & & 0.810 & 0.958 & 27.167 \\
\hline C_STH_U.J & & & & STSL MAN & & & 0.077 & 0.013 & 4.216 \\
\hline CI $\mathrm{S}+\mathrm{H} 1$ & 79 & 89 & 300 & FZCN_MAN, & & & 0.839 & 0.962 & 23.914 \\
\hline C_sth_1 & & & & STSL MAN & & & 0.054 & 0.009 & 3.349 \\
\hline $\mathrm{CI} \mathrm{S}+\mathrm{H} 2$ & 60 & 78 & 266 & FZCN_MAN, & & & 0.843 & 0.962 & 19.424 \\
\hline & & & & STSL_MAN & & & 0.047 & 0.010 & 2.713 \\
\hline CI $\mathrm{S}+\mathrm{H} \quad 0.5$ & 93 & 92 & 316 & $\overline{\text { FZCN_AUT, }}$ & & & 0.666 & 0.905 & 38.563 \\
\hline & & & 310 & STSL AUT & & & 0.087 & 0.019 & 4.147 \\
\hline $\mathrm{CI} \mathrm{S}+\mathrm{H} 1$ & 78 & 89 & 300 & FZCN_AUT, & & & 0.730 & 0.920 & 33.259 \\
\hline Cl_s+H_1 & 18 & 89 & 300 & STSL AUT & & & 0.061 & 0.018 & 3.508 \\
\hline CI_S+H_2 & 62 & 79 & 266 & FZCN_AUT, & & & 0.691 & 0.905 & 30.945 \\
\hline & & & & STSL AUT & & & 0.072 & 0.026 & 3.342 \\
\hline
\end{tabular}

* For the independent variables the prefix refers to the information extracted: STSL -standardized total length of hardwood stems; FZCN - fuzzy pixel count for shrub class. Postfixes MAN and AUT refer to manual and automated image extraction, respectively. For the dependent variable $\mathrm{Cl}: \mathrm{H}=$ hardwood, $\mathrm{S}=$ shrub for each height threshold $(\mathrm{m})$ given as the final character. Values in italics are the standard deviations from the 1000 resampling iterations.

Scatterplots for the competition index models with height threshold of $0.5 \mathrm{~m}$ are shown in

Figure 5.5.7. They reinforce the results given in Table 5.5.2, showing much lower residual spread around the modeled lines for the manual results than automated results.

Both manual and automated results fit the 1:1 line well, but errors for the automated 
method are larger towards the upper end of the observed data range for both shrub and hardwood competition.
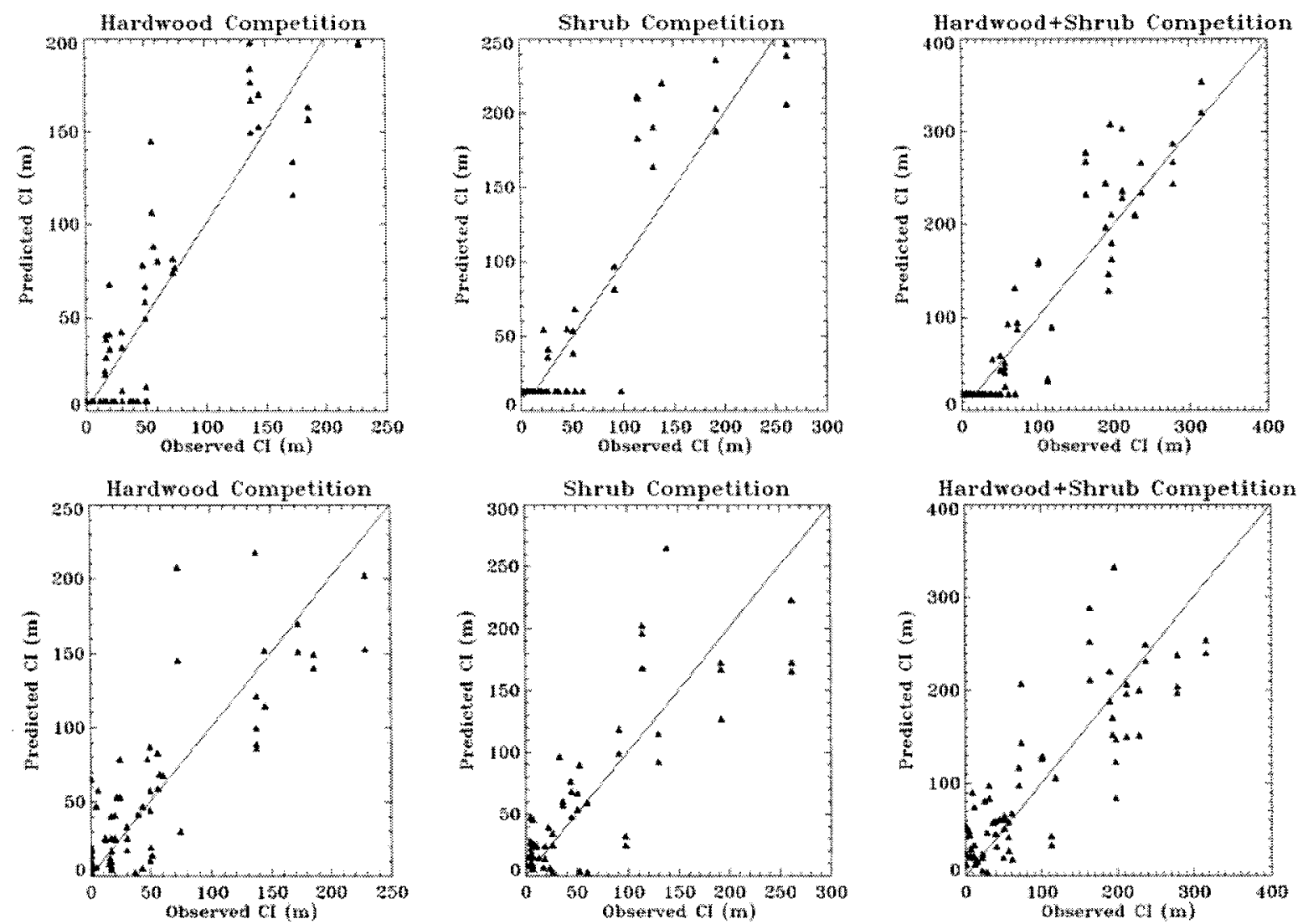

Figure 5.5.7: Scatterplots of hardwood and shrub competition indices estimated from imagery using manual (top) and automated (bottom) methods versus the field measured competition index for competition $>0.5 \mathrm{~m}$ in height. The solid $1: 1$ lines depict the relation $\mathrm{y}=\hat{\mathrm{y}}$.

\subsection{Competition Stem Density Modeling}

Table 5.5.3 shows the modeling results for competition stem density. Again, the manual results outperform the automated extraction, but similar trends were seen. The height thresholds had a strong effect on model quality. For hardwood competition, larger height thresholds improved model prediction accuracy. For shrub competition, the best model was obtained with the $1 \mathrm{~m}$ threshold. At the $2 \mathrm{~m}$ threshold, model bias increased slightly, 
reducing the EF statistic. However, MAE decreased consistently with increasing height thresholds again due to the reduction in the number of non-zero cases.

Table 5.5.3: Model results for competition stem density (stems $\mathrm{ha}^{-1}$ ).

\begin{tabular}{|c|c|c|c|c|c|c|c|c|c|}
\hline Depen & dent Va & ariable & & Independent & Model & ameters & & el Fit Sa & ics \\
\hline & Mean & Stdv & Max & Variable(s) & Slope & Offset & EF & BIAS & MAE \\
\hline SD $\mathrm{H} 0.5$ & 5378 & 6497 & 29360 & STSL MAN & 257.5 & 687.9 & 0.460 & 12.82 & 3182.66 \\
\hline SD_n_0.J & (30/0 & 0491 & 29500 & SISL_IVIAIN & 27.9 & 330.4 & 0.227 & 1053.82 & 434.54 \\
\hline $\mathrm{SD} \mathrm{H} 1$ & 4970 & 6063 & 26530 & ISTSI MAN & 239.1 & 608.0 & 0.574 & -0.59 & 2668.05 \\
\hline SD_H_1 & $49 / 0$ & 6063 & 26530 & [SISL_MAN & 23.9 & 283.3 & 0.169 & 874.97 & 363.43 \\
\hline $\mathrm{SD} \mathrm{H} 2$ & 3578 & 4982 & 19455 & STSL MAN & 186.7 & 163.4 & 0.816 & -16.40 & 1316.55 \\
\hline SD_11_2 & 3510 & 4902 & $134 J 3$ & DOSL_IVIAN & 14.6 & 145.2 & 0.056 & 465.54 & 194.89 \\
\hline $\mathrm{SD} \mathrm{H} 0.5$ & 5478 & 6593 & 29360 & STSL AUT & 263.5 & -107.5 & 0.453 & 30.96 & 2917.46 \\
\hline & & & & BISL_AUI & 31.8 & 317.5 & 0.238 & 1076.67 & 453.35 \\
\hline $\mathrm{SD} \mathrm{H}$ & 5078 & 6148 & & STSL AUT & 245.0 & -130.7 & 0.533 & 27.22 & 2553.28 \\
\hline SD_H_I & 5078 & 6148 & 26530 & STSL_AUT & 27.5 & 281.8 & 0.202 & 915.63 & 383.41 \\
\hline $\mathrm{SD} \mathrm{H} 2$ & 3686 & 5048 & 19455 & STSL AUT & 191.4 & -409.8 & 0.662 & 20.08 & 1805.24 \\
\hline & & & & & 20.0 & 182.9 & 0.132 & 638.32 & 236.10 \\
\hline SD S 0.5 & 11709 & 12551 & 47046 & IFZCN MAN & 304.8 & 4177.6 & -0.040 & 445.70 & 7893.13 \\
\hline SD_S_0.J & 11709 & 12531 & $4 / 040$ & FLCN_MAN & 48.3 & 795.5 & 0.471 & 3210.77 & 1658.58 \\
\hline SD $\mathrm{s} 1$ & 5225 & 8725 & 32807 & FZCN MAN & 175.6 & 741.5 & 0.834 & 21.65 & 2185.97 \\
\hline SD_S_1 & & $0 / 23$ & 32091 & 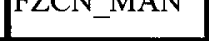 & 14.6 & 250.9 & 0.075 & 829.34 & 365.27 \\
\hline $\mathrm{SD} S 2$ & 2772 & 6568 & 26884 & FZCN MAN & 120.6 & -349.3 & 0.812 & -55.69 & 1466.41 \\
\hline SD_S_2 & $21 / 2$ & 0508 & 20884 & |FLCN_MAIN & 14.9 & 160.0 & 0.089 & 614.15 & 242.56 \\
\hline & & & 47046 & & $\overline{339.4}$ & 945.0 & 0.057 & 99.75 & $\overline{7480.56}$ \\
\hline SD_S_0.5 & 12032 & 12680 & 47046 & FLCN_AUT & 52.9 & 691.6 & 0.399 & 2939.66 & 1451.76 \\
\hline $\mathrm{SD} \mathrm{S} 1$ & 5399 & 8849 & 32897 & FZCN AUT & 196.9 & -1102.1 & 0.729 & -55.30 & 3108.73 \\
\hline$S D \_S 1$ & 5399 & 8849 & 52891 & FZCN_AUI & 22.0 & 369.2 & 0.088 & 986.10 & 353.98 \\
\hline $\mathrm{SD} \mathrm{S} 2$ & 2881 & 6675 & 26884 & & 135.7 & -1613.5 & 0.615 & -78.00 & 2597.29 \\
\hline & 2881 & $00 / 3$ & 20804 & FLCN_AU1 & 21.3 & 3460 & 0.160 & 867.77 & 292.16 \\
\hline & & & & FZCN_MAN, & & & 0.042 & 0.82 & 8444.24 \\
\hline SD_H + S_0.5 & 17109 & 13743 & 49522 & STSL MAN & & & 0.477 & 0.04 & 1744.75 \\
\hline $\mathrm{SD} \mathrm{H}+\mathrm{S} 1$ & 10204 & 10768 & 34312 & FZCN_MAN, & & & 0.767 & 0.95 & $\overline{3539.48}$ \\
\hline & & & & STSL MAN & & & 0.081 & 0.01 & 547.06 \\
\hline $\mathrm{SD} \mathrm{H}+\mathrm{S} 2$ & 6349 & 8272 & 28299 & FZCN_MAN, & & & 0.802 & 0.95 & 2303.82 \\
\hline SD_HTS_2 & 0349 & $82 / 2$ & 28299 & STSL MAN & & & 0.060 & 0.01 & 333.39 \\
\hline & & & & FZCN_AUT, & & & 0.100 & 0.78 & 8381.16 \\
\hline $\mathrm{SD} \_\mathrm{H}+\mathrm{S} \_0.5$ & 17533 & 13836 & 49522 & STSL AUT & & & 0.358 & 0.05 & 1524.73 \\
\hline $\mathrm{SD} H+\mathrm{S} 1$ & 10486 & 10870 & 34312 & FZCN_AUT, & & & 0.724 & 0.92 & 4152.32 \\
\hline & & & & STSL AUT & & & 0.069 & 0.02 & 418.47 \\
\hline $\mathrm{SD} \mathrm{H}+\mathrm{S} 2$ & 6567 & 8360 & 28299 & FZCN_AUT, & & & 0.672 & 0.90 & 3464.00 \\
\hline $\mathrm{SD}$ _H+S_L & $656 /$ & 8360 & 28299 & STSL AUT & & & 0.069 & 0.02 & 324.59 \\
\hline
\end{tabular}

* For the independent variables the prefix refers to the information extracted: STSL -standardized total line length of hardwood stems; FZCN - fuzzy pixel count for shrub class. Postfixes MAN and AUT refer to manual and automated image extraction, respectively. For the dependent variable stem density (SD): $H=$ hardwood, $\mathrm{S}=$ shrub, for each height threshold $(\mathrm{m})$ given as the final character. Values in italics are the standard deviations from the 1000 resampling iterations. 
Figure 5.5.8 shows scatterplots for the stem density competition models at the $2 \mathrm{~m}$ height threshold. The $2 \mathrm{~m}$ threshold is shown because it produced the best overall result for stem density. The manual line fits appear suitable, without strong biases. However, as with the competition index, larger errors are seen for larger observed values in the automated results.
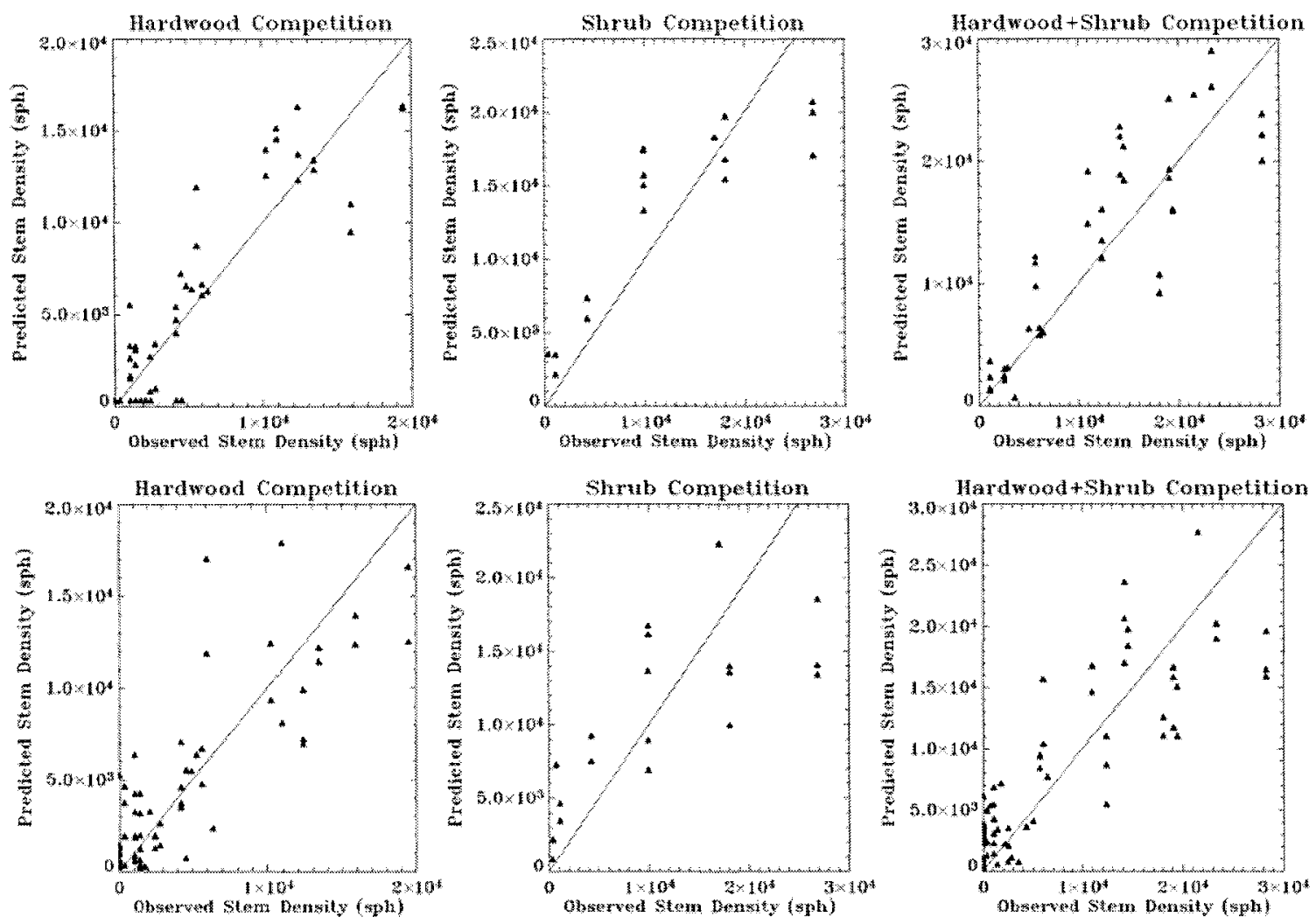

Figure 5.5.8: Scatterplots of hardwood and shrub competition stem density (stems per hectare, stems ha ${ }^{-1}$ ) estimated from imagery using manual (top) and automated (bottom) methods versus field measured competition stem density for competition $>1 \mathrm{~m}$ in height. The solid $1: 1$ lines depict the relation $\mathrm{y}=\hat{\mathrm{y}}$.

\subsubsection{Discussion}

This research was largely exploratory and intended to determine if there was potential for automated competition assessment using very high resolution leaf-off imagery. As the first known attempt at this, and to serve as a baseline for further research, the methods 
used were adapted from common image processing methods. The results showed that for the site conditions and image data of this study, strong relations exist between image and field measures of competition. The automated estimates compared favorably with the manual estimates, but had larger error, particularly where no competition was present, or in the upper range of competition abundance. Although generalization to other boreal conditions cannot be made from this initial study, the methods indicate good potential for further development in treatment decision making. For example, from the results of this study, manual competition assessment would produce a mean error for estimates of stem density above $2 \mathrm{~m}$ of about 1300 stems ha $^{-1}$ for hardwoods, 1500 stems ha $^{-1}$ for shrubs, and 2300 stems $^{-1} a^{-1}$ for shrubs and hardwoods combined. If an approximate threshold for the decision to apply a competition control treatment is 5000 stems $^{-1} \mathrm{a}^{-1}$ of hardwood and shrub competition (Fox, 2005 pers. comm.), it appears that the results of the manual extraction could be used to identify areas meeting this criteria. For the automated results, errors in competition stem density would be $\sim 1.5$ times larger, reducing the precision of such a treatment threshold estimate. A potential solution to combine the positive attributes of each type of analysis would be to use automated analysis to process the bulk of the data, with manual interpretation or field based surveys used to evaluate areas identified from the automated results to be within \pm 2300 stems $^{-1}$ of the desired competition threshold.

The above statements assume that both the automated and manual results in this study could be replicated in similar forest conditions. To better evaluate the potential advantage of manual interpretation over automated analysis, results of several 
independent interpreters should be used in an analysis of precision and accuracy. This should be combined with a relative cost analysis for evaluation of operational potential. Possible improvements to the manual extraction method are limited, but delineation of hardwood stems and interpretation of shrub areas would improve if conducted in a stereo workstation environment. This however, would require additional image acquisition, storage and processing time that would add to an already laborious task. Automated extraction provides the capability for rapid image processing, but improvements are required for it to be comparable to manual extraction. For automated processing, classification was the most important component affecting accuracy, as it was used as a direct measure of shrub competition and to constrain candidate lines for hardwood competition. Other automated methods may be able to aid in extracting the required information and need to be explored. For example, there are numerous other approaches to texture characterization that could improve results, especially methods that evaluate texture over multiple scales based on fractal analysis (Emerson et al., 1999), wavelets (Zhu and Yang, 1998), or co-occurrence texture (Coburn and Roberts, 2004). The use of other classification methods that incorporate spatial properties, such as contextual classification (Gong and Howarth, 1992), neural networks, or object-oriented classification should also be investigated.

The measure of competition to be modeled is an important operational consideration. The competition index was more strongly related to image measurements than stem density and would improve the ability to determine whether a given competition threshold has been met. However, the competition index values do not have direct meaning and would 
require calibration to be used in decision-making. A problem with both measures of competition is that plots with a large number of small competitors could have similar values to plots with a small number of large stems. The influence of these two conditions on crop tree growth is different and important to identify. The use of a height threshold as presented in this study helps to reduce such error and was warranted for the given vegetation age and structure in the sites. Other competition indices could also be evaluated that may improve the models.

The problem of woody debris being oriented in a similar direction to that of the relief displaced woody stems presents a major difficulty for the automated methods. One approach to help reduce this source of error is to include stem width and use a threshold to distinguish competition from woody debris. However, in this dataset, woody debris were in some cases of similar width to the competition. As a simple practical solution, for image plots identified with high competition, a visual scan could be conducted to assess whether woody debris is a substantial source of error.

The long-term objective of this research is to develop an efficient and robust remote sensing methodology for monitoring forest regeneration under basic and extensive silviculture management. The high resolution sampling approach described here was intended to provide detailed and accurate information on competition from leaf-off imagery. Combining this with automated methods for coniferous crop tree detection, delineation and measurement (Pouliot et al., 2002; Pouliot and King, 2005; and Pouliot et al., 2005) would provide capability for integrated regeneration assessment. The results 
could then be aggregated to strata level estimates following the procedure currently used for plot based field sampling for regeneration assessments. Further research will evaluate potential improvements to the methods developed here as well as other sensors such as lidar or radar that may help to identify differences between ground covers and competition. Most important is evaluation with a dataset of greater coverage to more fully identify conditions where the methods succeed or fail and the frequency with which such conditions can be expected to occur in a more operational setting.

\subsubsection{Conclusion}

Using a combination of spectral, textural and linear object information automatically extracted from leaf-off very high-resolution imagery, strong models were produced for shrub, hardwood, and combined shrub +hardwood regeneration competition estimation. As this is the first study of its kind, common automated methods were selected to serve as a baseline for further research. Further refinement of the methodology should improve model quality. The study also included competition estimates derived from a single manual interpretation that were slightly more accurate than the automated estimates. It is proposed that automated methods be used in a first pass to provide maps of areas of high and low competition (requiring obvious treatment or no need for treatment), followed by manual interpretation of the more uncertain areas to determine if some of them also need treatment. Further research to determine operational potential should emphasize this combined methodology and include testing of several interpreters and a larger dataset. The ability to evaluate competition abundance from high-resolution imagery coupled with automated conifer tree detection and delineation analysis greatly increases 
regeneration assessment capabilities and renders the costs associated with a remote sensing approach more favorable.

\subsubsection{Acknowledgements}

This research was funded by grants to D. King and scholarships to D. Pouliot from the Natural Sciences and Engineering Research Council of Canada. The authors would like to thank Mark Lindsay, Wayne Bell, Duncantech Ltd. (now Redlake Inc.), Wiskair Ltd., and Buchanan Forest Products Ltd. for their contributions to this research. 


\section{SUMMARY AND CONCLUSIONS}

The purpose of this research was to develop the means to estimate conifer crop and woody competition vegetation attributes from single date remote sensing imagery. Leafoff high resolution imagery was used as it was considered optimal for crop tree information and provided some potential to evaluate woody competition abundance. High resolution imagery was required so that small trees could be detected and to provide spatial estimates of crop and competition attributes. Single date imagery was required to minimize costs and facilitate potential operational adoption of the developed methods.

Three different study areas were used in the research. The first was an experimental study site where tree size, spacing, and species were controlled representing ideal conditions for evaluation of crop tree detection and delineation performance. The second study area consisted of three young cutovers with varying levels of competing vegetation representing more operational conditions for evaluation. The third study area consisted of three older cutovers and was used as an example operational application of crop tree assessment.

For crop tree assessment, an automated tree crown detection and delineation algorithm was developed based on identification of unique radiometric tree crown morphological attributes. For woody competition assessment a visual and automated identification procedure was developed that relied on linear feature morphology and spectral 
characteristics. The following sections discuss the key processing considerations and summarize accuracies for the analyses undertaken in this research.

\subsection{AUTOMATED CONIFER TREE DETECTION AND DELINEATION}

\subsubsection{Detection}

The algorithm developed for automated detection and delineation consisted of four stages: 1) preprocessing, 2) scale selection, 3) initial detection and delineation, and 4) delineation refinement. The first two stages have a significant influence on detection accuracy and should be considered carefully. In the pre-processing stage conifer pixels in the imagery need to be separated from other image features. This is best accomplished using an image classification technique, but simple thresholding can be sufficient for many applications. Colour infrared imagery is preferred as local maxima representing tree crowns are typically most distinct in the near-infrared wavelengths. However, the green wavelength has been used successfully so colour imagery may also be used. The second stage requires determination of the optimal scale for processing. Initially, in unsmoothed imagery, many maxima are detected due to noise and multiple branching structures within crowns, resulting in low accuracy. As image smoothing is increased to represent coarser scale image information, the number of detected maxima decreases rapidly, and then levels off. The method found to be best in this research (from a comparison of four methods) uses this relation to determine the point where the change in the number of detected local maxima slows (i.e. where the slope of the relation decreases) and becomes relatively constant with additional smoothing. Comparison of this relation 
for several datasets revealed that it is preferable to overestimate the required level of smoothing, as error changes more slowly for over-estimates of the optimal scale compared to under-estimates. It is also suggested that, in determining the optimal smoothing level, a set of samples from visual tree detection be used as a subsequent quality check. Further, this processing can be applied within more homogenous image subsets to improve results.

Based on the results for the different study areas, individual tree detection accuracy ranged from $47-87 \%$ with a mean of $70 \%$ using an accuracy index that combines both omission and commission error. At the plot level, the mean absolute error (MAE) ranged from $9-47 \%$ with a mean of $26 \%$ for stem counts. The best results were obtained for the controlled study site, as these conditions minimized omission error due to small trees or trees being hidden from the sensor view. The worst results were obtained for the young cutover site with the smallest trees. At this site, error was high because: 1) numerous small trees were not well resolved in the imagery, 2) many were hidden from the sensor by overtopping or neighbouring trees, 3 ) the crown size variability made it difficult to identify an optimal scale for both the small and large crowns in the imagery, and 4) low lying vegetation (green mosses) on the site led to commission errors in wet areas.

If only trees greater than a given minimum size threshold are considered, accuracy is much higher because omission errors associated with small trees and commission errors associated with green mosses or large crowns are reduced. This finding has been reported by Hall (1984) and Pitt and Glover (1993) in other regeneration research using 
photography. Both studies also found that not including small trees $(<35 \mathrm{~cm})$ in visual stem counting greatly improved accuracy. If management and lor sampling objectives require only detection of trees above a certain size threshold, the omission of such small trees may be inconsequential. Another factor to consider, that has not yet been addressed, is the potential improvement in tree detection estimates if only well-spaced trees are counted instead of total stem density. Such estimates would only include trees separated by a given minimum distance, reducing the error associated with overlapping or hidden trees.

\subsubsection{Delineation}

Delineation is carried out using a watershed algorithm to identify initial crown boundaries, which are then refined by local edge detection. The refinement helps to better identify the crown boundary based on local spatial properties, whereas the initial boundary is determined from a global spectral operation such as thresholding or classification. The MAE in diameter of delineated crowns for individual trees ranged from $9-23 \%$ with a mean of $17 \%$. Plot level MAE ranged from $8-27 \%$ with a mean of $20 \%$ for plot average crown diameter. The best results were obtained for the experimental study site because of the ideal conditions for detection and delineation. Based on individual tree comparison, the worst results were observed for the young cutover with high competition due to numerous commission errors in larger crowns (more than one polygon delineated in a crown) causing crown sizes to be underestimated. For the plot level comparison detection error was again a significant contributor to delineation error. In the young cutover low competition site, omission error of small trees 
resulted in a large difference between field and image measured average crown diameters. Overall, the old cutover sites consistently produced the largest errors as they had regenerating structures that were difficult for detection and the aerial photography used in that project was subject to strong geometric, sun-view angle brightness, and topographic distortions as well as scanned pixel size limitations. Considering only trees detected correctly (i.e. 1 image segment to 1 reference segment), error was reduced to less than $20 \%$, suggesting that if accurate detection can be achieved then delineation error will improve. Competing vegetation has also been found to moderately affect crown delineation accuracy. High levels of woody stem competition cause crown boundaries to be less distinct. However, the effect does not appear to be more significant than other factors that influence crown boundary delineation, such as detection error.

\subsubsection{Effects of Imaging Conditions on Detection and Delineation}

Imaging conditions were shown to strongly influence detection and delineation results. In the old cutover study area, error in both detection and delineation was found to increase non-linearly with view angle. Leckie et al. (2005) found a $\sim 10 \%$ difference in detection results due to sun-sensor geometry. Using simulated imagery of mature eucalyptus forests, Culvenor (2000) showed that tree detection accuracy was best for small off nadir view angles $\left(<15^{\circ}\right)$, with back scattered as opposed to forward scattered radiance, and at higher solar zenith angles. Thus, detection should be carried out within narrow view angles for optimal results. The effects of topography have not been widely researched. Culvenor et al. (2000) found that they depend on the incident radiation relative to the topographic slope and aspect. At the tree level, differences in illumination due to 
topography cause differences in mutual shading of crowns and the brightness response of the background. At a coarser scale, topography produces shaded and sunlit slopes that make it difficult to effectively mask crowns for automated analysis. Topography also affects pixel size and thus can lead to errors in crown size estimates, but with suitable ortho-rectification it is expected that this error can be sufficiently reduced.

\subsection{NON-CROP COMPETITION EXTRACTION}

The methodology developed for woody deciduous competition extraction is the first known attempt using leaf-off imagery. For this reason, more commonly used image processing techniques were explored to determine the potential for this type of analysis and to determine where improvements were most needed. They included spectral and textural classification as well as line detection of hardwood competition stems. The methodology requires very high resolution imagery $(\sim 2 \mathrm{~cm})$ to capture the spatial properties of the competition stems and branches. For hardwoods, view angles greater than $6^{\circ}$ are needed so that stems are clearly visible. True colour imagery is recommended because visual analysis of hardwood and shrub stems revealed that they were most distinct with this wave-band combination.

The results showed that useful relations were obtained for both visual and automated methods, but the visual method was superior. Thus, for current operational practice it is recommended that the automated approach be used first to stratify a cutover into: 1) areas where little competition exists so they can be removed from consideration for silvicultural treatment; 2) areas with obviously high levels of competition that can be 
flagged for treatment; and 3) areas where competition is close to a given threshold required for silvicultural treatment decisions. The latter can then be further evaluated using visual assessment to provide a more accurate estimate of competition.

Numerous avenues exist for improvement of the current methodology. Classification was the most important component affecting accuracy, as it was used as a direct measure of shrub competition and to constrain candidate lines for hardwood competition. Other more advanced classification methods should be explored such as neural networks (Benediktsson et al., 1990), contextual classification (Gong and Howarth, 1992), evidence based classification (Latifovic and Pouliot, 2005), or object oriented classification (Definiens, 2003). The incorporation of spatial transforms is also another potential means to improve classification. As spatial properties are often scale dependent, methods that evaluate texture over multiple scales may be the most appropriate; examples include fractal analysis (Emerson et al., 1999), wavelets (Zhu and Yang, 1998), or multiscale co-occurrence analysis (Coburn and Roberts, 2004). Coarse woody debris is also a source of error for hardwood extraction. This is a difficult problem as hardwood stems and coarse woody debris have similar spectral and morphologic characteristics. A potential improvement, not exploited in this research, is to estimate the stem width, which can be used to separate larger woody debris from competition stems. 


\section{REFERENCES}

Alberta Sustainable Resource Development (2003). Alberta regeneration survey. Forest Management Branch, Edmonton, Alberta, Canada. ISBN 0-7785-2909-6, 96 pp.

Apsey, M., D. Laishley, V. Nordin, and G. Paille (2000). The perpetual forest: using lessons from the past to sustain Canada's forests in the future. Forestry Chronicle, 76: $29-53$.

Archibold, O.W. (1995). Ecology of world vegetation. Chapman and Hall, London, 499 pp.

Auclair, A.N.D., and J.A. Bedfors (1997). Century trends in the volume balance of boreal forests: implications for global $\mathrm{CO}_{2}$ balance, in Global change and arctic terrestrial ecosystems. Edited by W.C. Oechel, T. Callaghan, T. Gilmanov, J.I. Holten, B. Maxwell, U. Molau, and B. Sveinbjornsson. Springer, New York, Ecological Studies, 124: 452-472.

Beckley, T.M. (1999). Forest community sustainability: introduction to the theme. Forestry Chronicle, 75: 745-747.

Bell, F.W. (2001). Forest vegetation management specialist, Ontario Forest Research Institute. Ontario Ministry of Natural Resources. Personnel communication.

Bell, F.W., M.T. Ter-Mikaelian, and R.G. Wagner (2000). Relative competitiveness of nine early successional boreal forest species associated with planted jack pine and black spruce seedlings. Canadian Journal of Forest Research, 30: 790-800.

Benediktsson, J.A., H.P. Swain, and K.O. Ersoy (1990). Neural network approaches versus statistical methods in classification of multisource remote sensing image classification. IEEE Transactions on Geoscience and Remote Sensing, 37:13501359.

Brandtberg, T., T.A. Warner, R.E. Landenberger, and J.B. McGraw (2003). Detection and analysis of individual leaf-off tree crowns in small footprint, high sampling density lidar data from the eastern deciduous forest in North America. Remote Sensing of Environment, 85: 290-303.

Brandtberg, T. (1999). Algorithms for structure- and contour-based tree species classification using digital image analysis, in Proceedings of the International Forum on Automated Interpretation of High Spatial Resolution Digital Imagery for Forestry. 10-12 February 1998, Victoria, B.C. Pacific Forestry Centre, Canadian Forest Service, Natural Resources Canada, Victoria, B.C. pp. 199-205. 
Brandtberg T., and F. Walter (1998). Automated delineation of individual tree crowns in high spatial resolution aerial images by multiple-scale analysis. Machine Vision and Applications, 11: 64-73.

British Columbia Forest Practice Branch (2002). Stocking and free growing survey procedures manual. British Columbia Forest Practice Branch, 119 pp.

Brown, R., and V. Fletcher (1999). Application of CASI remote sensing to classification of backlog not satisfactorily restocked forest in northern British Columbia, in Proceedings of the International Forum on Automated Interpretation of High Spatial Resolution Digital Imagery for Forestry. 10-12 February 1998, Victoria, B.C. Pacific Forestry Centre, Canadian Forest Service, Natural Resources Canada, Victoria, B.C. pp. 155-160.

Burton, P.J. (1993). Some limitations inherent to static indices of plant competition. Canadian Journal of Forest Research, 23: 2141-2151.

Butson, C.R., and D.J. King (2001). Determination of optimal forest sample plot spatial extent using lacunarity analysis of airborne imagery. Proceedings 18th Biennial Workshop on Colour Photography \& Videography in Resource Assessment (Am. Soc. Photogramm. And Rem. Sens.), Amherst, Massachusetts, May 16-18. CDROM publication.

Callan, B. (2001). Introduction to forest diseases. ISBN 0-662-30780-1. Natural Resources Canada, Canadian Forest Service, $14 \mathrm{pp}$.

Campbell, J.B. (1996). Introduction to remote sensing, second edition. Guilford Press, $607 \mathrm{pp}$.

Caza, C.L., and D. Neave (2000). New millenium forestry and the fate of wildlife. Forestry Chronicle, 76: 109-115.

Chang, M. (2003). Forest hydrology: an introduction to water and forests. CRC Press, $373 \mathrm{pp}$.

Chen, J.M., G. Pavlic, L. Brown, J. Cihlar, S.G. Leblanc, H.P. White, R.J. Hall, D.R. Peddle, D.J. King, J.A. Trofymow, E. Swift, J. Van der Sanden, and P.K.E. Pellikka (2002). Derivation and validation of Canada-wide coarse-resolution leaf area index maps using high-resolution satellite imagery and ground measurements. Remote Sensing of Environment, 80: 165-184.

Chen, J.M., W. Chen, J. Liu, and J. Cihlar (2000). Annual carbon balance of Canada's forests during 1895-1996. Global Biogeochemical Cycles, 14: 839-849. 
Coburn, C.A., and A.C.B. Roberts (2004). A multiscale texture analysis procedure for improved forest stand classification. International Journal of Remote Sensing, 25: 4287-4308.

Coleman, T.L., L. Gudapati, and J. Derrington (1990). Monitoring forest plantations using Landsat Thematic Mapper Data. Remote Sensing of Environment, 33: 211221.

Comeau, P.G., T.F. Braumandl, and C. Xie. (1993). Effects of overtopping vegetation on light availability and growth of Engelmann spruce (Picea engelmannii) seedlings. Canadian Journal of Forest Research, 23: 2044-2048.

Culvenor, D.S. (2003). Extracting individual tree information : a survey of techniques for high spatial resolution imagery, in Remote sensing of forest environments: concepts and case studies. Edited by M.A. Wulder and S.E. Franklin. Boston: Kluwer Academic Publishers, pp. 255-277.

Culvenor, D.S. (2000). Development of a tree delineation algorithm for application to high spatial resolution digital imagery of Australian native forest. Ph.D. Thesis, University of Melbourne, $355 \mathrm{pp}$.

Culvenor, D.S., N. Coops, R. Preston, and K.G Tolhurst (1999). A spatial clustering approach to automated tree crown delineation, in Proceedings international forum on automated interpretation of high spatial resolution digital imagery for forestry. Edited by D. A. Hill and D. G. Leckie. Victoria, B.C. Pacific Forestry Centre, Canadian Forest Service, Natural Resources Canada, Victoria, B.C. pp. 67-80.

Curran, P.J. (1988). The semivariogram in remote sensing: an introduction. Remote Sensing of Environment, 24: 493 - 507.

Definiens-Imaging (2003). www.definiens-imaging.com. Accessed March 21, 2004.

Delaney, Jeff, T. Ringash, and D. Auer (2000). Manitoba forest regeneration survey manual. Manitoba Conservation, Forestry Branch, Forest Health and Ecology, Winnipeg, $39 \mathrm{pp}$.

Diniz-Filho, J.A.F., L.M. Bini, and B.A. Hawkins (2003). Spatial autocorrelation and red herrings in geographical ecology. Global Ecology and Biogeography, 12:53-64.

Dralle, K., and M. Rudemo (1997). Automatic estimation of individual tree positions from aerial photos. Canadian Journal of Forest Research, 27: 1728-1736.

Dralle, K., and M. Rudemo (1996). Stem number estimation by kernel smoothing in aerial photos. Canadian Journal of Forest Research, 26: 1228-1236. 
Draper, N., and H. Smith (1998). Applied regression analysis: 3rd edition. John-Wiley and Sons Inc. $736 \mathrm{pp}$.

Du, Y., J. Cihlar, and R. Latifovic (2001). Radiometric normalization compositing, and quality control for satellite high resolution image mosaics over large areas. IEEE transactions on Geoscience and Remote Sensing, 39: 623-634.

Duchesne, L.C., J.C. Zasada, and I.Davison-Hunt (2000). Nontimber forest products industry in Canada: scope and research needs. Forestry Chronicle, 76: 743-746.

Emerson, C.W., N.S. Lam, and D. Quattrochi (1999). Multi-scale fractal analysis of image texture and pattern. Photogrammetric Engineering and Remote Sensing, 65: 51-61.

Environment Canada (2004). Threats to water availability in Canada. National Water Research Institute, Burlington, Ontario. NWRI Scientific Assessment Report Series No. 3 and ACSD Science Assessment Series No. 1. 128 pp.

Erikson, M. (2004). Species classification of individually segmented tree crowns in highresolution aerial images using radiometric and morphologic image measures. Remote Sensing of Environment, 91: 469-477.

Erikson, M. (2003). Segmentation of individual tree crowns in colour aerial photographs using region growing supported by fuzzy rules. Canadian Journal of Forest Research, 33: 1557-1563.

Farrar, J.L. (1995). Trees in Canada. Fitzhenry and Whiteside Ltd. Markham, Ontario, Canada, 502 pp.

Fiorella, M., and W.J. Ripple (1993). Analysis of conifer forest regeneration using Landsat Thematic Mapper data, Photogrammetric Engineering and Remote Sensing, 59: 1288-1382.

Foster, R.F. (1998). Grazing animals for forest vegetation management. Northwest Science and Technology. Technical Note TN-40, in Vegetation management alternatives - guide to opportunities. Edited by F.W. Bell, M. McLaughlan, and J. Kerley. Ontario Ministry of Natural Resources, Thunder Bay, Ont. 10 pp.

Fox., B. (2004). Forest health and silviculture specialist. Ontario Ministry of Natural Resources. Personnel communication.

Franklin, S.E., D.R. Peddle, J.A. Dechka, and G.B. Stenhouse (2002). Evidential reasoning with Landsat TM, DEM and GIS data for landcover classification in support of grizzly bear habitat mapping. International Journal of Remote Sensing, 23: 4633-4652. 
Franklin, S.E., R.J. Hall, L.M. Moskal, A.J. Maudie, and M.B. Lavigne (2000). Incorporating texture into classification of forest species composition from airborne multispectral images. International Journal of Remote Sensing, 21: 61-79.

Fraser, R., A. Abuelgasim, and R. Latifovic (2005). A method for detecting large-scale forest cover change using coarse spatial resolution imagery. Remote Sensing of Environment, 30: 414-427.

Gillis, M.D., and D.G. Leckie (1996). Forest inventory update in Canada. The Forestry Chronicle, 72: 138-156.

Goba, N., S. Pala, and J. Narraway (1982) An instruction manual on the assessment of regeneration success by aerial survey. Ontario Ministry of Natural Resources. Toronto, Ont. 57 pp.

Gong, P., and P.J. Howarth (1992). Frequency-based contextual classification and graylevel vector reduction for land-use identification. Photogrammetric Engineering and Remote Sensing, 58: 423-437.

Gonzalez, R.C., and R.E. Woods (2002). Digital image processing, second edition. Pearson Education Ltd. 793 pp.

Gougeon, F.A., and D.G. Leckie (1999). Forest regeneration: individual tree crown detection techniques for density and stocking assessments, in Proceedings international forum on automated interpretation of high spatial resolution digital imagery for forestry. Edited by D. A. Hill and D. G. Leckie. Victoria, B.C. Pacific Forestry Centre, Canadian Forest Service, Natural Resources Canada, Victoria, B.C. pp. 169-177.

Gougeon, F.A., and T. Moore (1988). Individual tree classification using MEIS-II imagery. Page 927 in Proc. 1988 Intl. Geoscience and Remote Sensing Symposium (IGARSS'88). Edinburgh, Scotland, September 13-16, 1988.

Gougeon, F.A. (1997). A locally adaptive technique for forest regeneration assessments from high resolution aerial images. CD-ROM of Proceedings of the 19th Symposium on Remote Sensing, May 24-30, 1997, Ottawa, Ontario, Canada. Canadian Aeronautics and Space Institute, Ottawa.

Gougeon, F.A. (1995a). A crown-following approach to the automatic delineation of individual tree crowns in high spatial resolution images. Canadian Journal of Remote Sensing, 21: 274-288.

Gougeon, F.A. (1995b). Comparison of possible multispectral classification schemes for tree crowns individually delineated on high spatial resolution MEIS Images. Canadian Journal of Remote Sensing, 21:1-9. 
Greene, D.F., J.C. Zasada, L. Sirois, D. Kneeshaw, H. Morin, I. Charron, and M.J. Simard (1999). A review of the regeneration dynamics of North American boreal forest tree species. Canadian Journal of Forest Research, 29: 824 - 839.

Grose, A.J. (1995). Non-timber dimensions of forest management: a qualitative evaluation of Ontario's forest policy. Carleton University, M.A. Thesis, 185 pp.

Haddow, K.A., D.J. King, D.A. Pouliot, D.G. Pitt, and F.W. Bell (2000). Early regeneration conifer identification and competition cover assessment using airborne digital camera imagery. Forestry Chronicle, 76: 915-928.

Hall, F., D. Botkin, D. Strebel, K. Woods, and S. Goetz (1991). Large-scale patterns of forest succession as determined by remote sensing. Ecology, 72: 628-640.

Hall, R.J., and A.H. Aldred (1992). Forest regeneration appraisal with large -scale aerial photographs. Forestry Chronicle, 68: 142-150.

Hall, R.J. (1984). Use of large-scale aerial photographs in regeneration assessments. Information Report NOR-x-264, Northern Forest Research Centre, Canadian Forest Service, $31 \mathrm{pp}$.

Hall-Beyer, M. (2004). GLCM texture: a tutorial.

http://www.ucalgary.ca/ mhallbey/texture\% 20tutorial/texture_tutorial.html. Accessed 10/09/2005.

Haralick, R.M., K. Shanmugam, and I. Dinstein (1973). Textural features for image classification. IEEE Transactions on Systems, Man, and Cybernetics SMC, 3: 610621.

Hay, G., T. Blaschke, D. Marceau, and A. Bouchard (2003). A comparison of three image-object methods for multiscale analysis of landscape structure. ISPRS Journal of Photogrammetry and Remote Sensing, 57: 327-345.

Hay, G., K. Niemann, and D. Goodenough (1997). Spatial thresholds, image -objects, and upscaling: A multiscale evaluation. Remote Sensing of Environment, 62: 1-19.

Hayward, C., and D. Slaymaker (2000). Estimating the significant above ground biomass of amazonian rain forest using low altitude aerial videography. Proc. 18th Biennial Workshop on Colour Photography \& Videography in Resource Assessment (Am. Soc. Photogramm. and Rem. Sens., Bethesda MD), Amherst, Massachusetts, May 16 - 18. CD-ROM publication.

Holmgren, J., and A. Persson (2003). Identifying species of individual trees using airborne laser scanner. Remote Sensing of Environment, 90: 415-423. 
Horler, D.N.H., and F.J. Ahern (1986). Forestry information content of Thematic Mapper data, International Journal of Remote Sensing, 7: 405-428.

Jakubauskas, M.E. (1997). Effects of forest succession on texture in Landsat Thematic Mapper imagery, Canadian Journal of Remote Sensing, 23: 257-263.

Jensen, J.R. (1996). Introductory digital image processing: a remote sensing perspective. Prentice Hall, New Jersey, 316 pp.

Kimmins, H. (1997). Balancing act: environmental issues in forestry, second edition. UBC Press, 305 pp.

King, D.J. (2000). Airborne remote sensing in forestry: sensors, analysis and applications. Forestry Chronicle, 76: 859-876.

King, D.J. (1995). Airborne multispectral digital camera and video sensors: a critical review of system designs and applications. Canadian Journal of Remote Sensing, 21: $245-273$.

Kurz, W.A., and M.J. Apps (1996). Retrospective assessment of carbon flows in Canadian boreal forests, in Forest ecosystems, forest management and the global carbon cycle. Edited by M.J. Apps and D.T. Price, NATO ASI Ser. I, 40, pp. 173182.

Larsen, J.A. (1980). The boreal ecosystem. Academic Press, 495 pp.

Latifovic, R., and D. Pouliot (2005). Multitemporal land cover mapping for Canada: methodology and products. Canadian Journal of Remote Sensing, 31:347-363.

Latifovic, R., Z. Zhu, J. Cihlar, C. Giri, and I. Olthof (2004). Land cover mapping of North and Central America - Global Land Cover 2000. Remote Sensing of Environment, 89: 116-127.

Lautenschlager, R.A., F.W. Bell, R.G. Wagner, and J.A. Winters (1997). The Fallingsnow ecosystem project: comparing conifer release alternatives in northwestern Ontario. Forestry Chronicle, 73: 35-38.

Laws, K.I. (1980). Rapid texture identification. SPIE Image Processing for Missile Guidance. pp. 376-380.

Leckie, D.G., F.A. Gougeon, S. Tinis, T. Nelson, C. Burnett, and D. Paradine (2005). Automated tree recognition in old-growth conifer stands with high-resolution digital imagery. Remote Sensing of Environment, 94: 311-326.

Leckie, D.G., C. Jay, F.A. Gougeon, R.N. Sturrock, and D. Paradine (2004). Detection and assessment of trees with Phellinus weirii (laminated root rot) using high 
resolution multispectral imagery. International Journal of Remote Sensing, 25: 793818.

Leckie, D.G., F.A. Gougeon, N. Walsworth, and D. Paradine (2003a). Stand delineation and composition estimation using semi-automated individual tree crown analysis. Remote Sensing of Environment, 85: 355-369.

Leckie, D., F. Gougeon, D. Hill, R. Quinn, L. Armstrong, and R. Shreenan (2003b). Combined high-density lidar and multispectral imagery for individual tree crown analysis. Canadian Journal of Remote Sensing, 29: 633-649.

Lévesque, J. and D.J. King (1999). Airborne digital camera image semivariance for evaluation of forest structural damage at an acid mine site. Remote Sensing of Environment, 68: 112-124.

Lillesand, T.M., and R.W. Kiefer (2000). Remote sensing and image interpretation, 4th Edition. John Wiley \& Sons, Toronto. 715 pp.

Lindeberg, T. (1994). Scale-space theory in computer vision. Kluwer Academic Publishing, Dordrecht, The Netherlands. 394 pp.

Manion, D.D. (1991). Tree disease concepts, second edition. Prentice Hall Career and Technology, $387 \mathrm{pp}$.

Mas, J. (1999). Monitoring land-cover changes: a comparison of change detection techniques. International Journal of Remote Sensing, 19: 245-265.

Mayer, D.G., and D.G. Bulter (1993). Statistical validation. Ecological Modeling, 68: 2132.

McDonald, P.M., and G.O. Fiddler (1993). Feasibility of alternatives to herbicides in young conifer plantations in California. Canadian Journal of Forest Research, 23: 2015-2022.

Morris, D.M., G.B. MacDonald, and K.M. McClain (1990). Evaluation of morphological attributes as response variables to perennial competition for 4-year-old black spruce and jack pine seedlings. Canadian Journal of Forest Research, 20: 1696-1703.

Natural Resources Canada (2004). State of Canada's forests: the boreal forest. Natural Resources Canada, $95 \mathrm{pp}$.

Natural Resources Canada (2000). The state of Canada's forests 1999-2000. Natural Resources Canada, ISBN 0-662-29069-0, 120 pp.

Niemann, K.O., S. Adams, and G. Hay (1999). Automated tree crown identification using digital orthophoto mosaics, in Proceedings international forum on automated 
interpretation of high spatial resolution digital imagery for forestry. Edited by D. A. Hill and D. G. Leckie. Victoria, B.C. Pacific Forestry Centre, Canadian Forest Service, Natural Resources Canada, Victoria, B.C. pp. 105-108.

Nyland, R. D. (1996). Silviculture concepts and applications. McGraw-Hill Companies, $633 \mathrm{pp}$.

Olthof, I., D.A. Pouliot, R. Fernades, and R. Latifovic (2005). ETM+ radiometric normalization comparison for northern mapping applications. Remote Sensing of Environment, 95: 388-398.

Ontario Environmental Assessment Board (1994). Reasons for decision and decision: class environmental assessment by the ministry of natural resources for timber management on crown lands in Ontario. Toronto, $535 \mathrm{pp}$.

Ontario Ministry of Natural Resources (2004). Well-spaced free-growing regeneration assessment procedure for Ontario. Draft manual for field testing. Ontario Ministry of Natural Resources, 42 pp.

Peddle, D.R., and S.E. Franklin (1991). Image texture processing and data integration for surface pattern discrimination. Photogrametric Engineering and Remote Sensing, 57: 413-420.

Pellikka, P.K.E., D. J. King and S.G. Leblanc (2000). Quantification and removal of bidirectional effects in aerial CIR imagery of deciduous forests using two reference land surface types. Remote Sensing Reviews, Special issue on "Multi-angle Measurements and Models", 19: 259-291.

Persson, A., J. Holmgren, and U. Soderman (2002). Detecting and measuring individual trees using an airborne laser scanner. Photogrammetric Engineering and Remote Sensing, 68: 925-932.

Pinto, F., D. Pearce, and D. Nesbitt (2003). Silviculture treatment assessment and reporting system (STARS): background and field manual. South Central Sciences and Information Section, Ontario Ministry of Natural Resources, $20 \mathrm{pp}$.

Pinz, A. (1999). Tree isolation and species classification, in Proceedings international forum on automated interpretation of high spatial resolution digital imagery for forestry. Edited by D. A. Hill and D. G. Leckie. Victoria, B.C. Pacific Forestry Centre, Canadian Forest Service, Natural Resources Canada, Victoria, B.C. pp. 127-139.

Pitkanen, J. (2001). Individual tree detection in digital aerial images by combining locally adaptive binarization and local maxima methods. Canadian Journal of Forest Research, 31: 832-844. 
Pitt, D.G., U. Runesson, and F.W. Bell (2000). Application of large- and medium-scale aerial photographs to forest vegetation management: a case study. Forestry Chronicle, 76: 903-913.

Pitt, D.G., M.T. Dumas, R.E. Wall, D.G. Thompson, L. Lanteigne, W. Hintz, G. Sampson, and R.G. Wagner (1999). Chondrostereum purpureum as a biological control agent in forest vegetation management. Efficacy on speckled alder, red maple, and aspen in eastern Canada. Canadian Journal of forest Research, 29: 841 851.

Pitt, D.G., R.G. Wagner, R.J. Hall, D.J. King, D. G. Leckie, and U. Runesson (1997). Use of remote sensing for forest vegetation management: a problem analysis. Forestry Chronicle, 73: 459-478.

Pitt, D.G., and G.R. Glover (1996). Measurements of woody plant attributes from largescale aerial photographs. New Zealand Journal of Forest Science, 26: 53-73.

Pitt, D.G., G.R. Glover and R.H. Jones (1996). Two-phase sampling of woody and herbaceous plant communities using large-scale aerial photographs. Canadian Journal of Forest Research, 26: 509-524.

Pitt, D.G., and G.R. Glover (1993). Large-Scale 35-mm aerial photographs for assessment of vegetation-management research plots in Eastern Canada. Canadian Journal of Forest Research, 23: 2159-2169.

Pollock, R. (1999). Individual tree recognition based on a synthetic tree crown image model. A spatial clustering approach to automated tree crown delineation, in Proceedings international forum on automated interpretation of high spatial resolution digital imagery for forestry. Edited by D. A. Hill and D. G. Leckie. Victoria, B.C. Pacific Forestry Centre, Canadian Forest Service, Natural Resources Canada, Victoria, B.C. pp. 25-34.

Pouliot, D.A., D.J. King, and D.G. Pitt (2006). Automated assessment of hardwood and shrub competition in regenerating forests using leaf-off airborne imagery. Remote Sensing of Environment. In press.

Pouliot, D.A., and D.J. King (2005a). Approaches for optimal automated individual tree crown detection in young regenerating coniferous forests. Canadian Journal of Remote Sensing, 31: 255-267.

Pouliot, D.A., and D.J. King (2005b). Towards the development of a remote sensing based coniferous regeneration monitoring system. Proceedings of the $20^{\text {th }}$ Biennial Workshop on Colour Photography and Videography in Resource Assessment, Weslaco, Texas, October 10-13, 8 pages. 
Pouliot, D.A., D.J. King, and D.G. Pitt (2005). Development and evaluation of an automated tree detection and delineation algorithm for monitoring regenerating coniferous forests. Canadian Journal of Forest Research, 35: 2332-2345.

Pouliot, D.A., and D.J. King (2004). Fallingsnow ecosystem study aerial photography: automated tree detection-delineation and vegetation classification. Report produced under the Collaborative Research Agreement Regarding A Joint Living Legacy Trust Grant, Between The Upper Lakes Environmental Research Network and Carleton University, $20 \mathrm{pp}$.

Pouliot, D.A., D.J. King, F.W. Bell, and D.G. Pitt (2002). Automated tree crown detection and delineation in high-resolution digital camera imagery of coniferous forest regeneration. Remote Sensing of Environment, 82: 322-334.

Price, R., and D. Davison. (1999). Operational techniques for assessing NSR areas using an airborne multispectral imager (CASI), in Proceedings international forum on automated interpretation of high spatial resolution digital imagery for forestry. Edited by D. A. Hill and D. G. Leckie. Victoria, B.C. Pacific Forestry Centre, Canadian Forest Service, Natural Resources Canada, Victoria, B.C. pp. 161-168.

Richards, J. A. (1993). Remote sensing digital Image analysis: an introduction, 2nd edition. Springer-Verlag New York, Inc. 340 pp.

Rowe, J. S. 1972. Forest regions of Canada. Pub. No. 1300. Canadian Forestry Service, Ottawa, $171 \mathrm{pp}$.

Saskatchewan Environment Forest Service (2004). Regeneration assessment. Saskatchewan Environment Forest Service, $42 \mathrm{pp}$.

Scarr, T.A., S.M. Smith, J.J. Turgeon, and G.M. Howse (2001). Chapter 6 stand tending and protection, insect pest management, in Regenerating the Canadian forest. Edited by R.G. Wagner and S.J. Colombo. Fitzhenry and Whiteside Ltd. Markham, Ontario, Canada, pp. 459-482.

Shaw, D.T., T.J. Malthus, and J.A. Kupiec (1998). High-spectral resolution data for monitoring Scots pines (Pinus sylvestris L.) regeneration. International Journal of Remote Sensing, 19: 2601-2608.

Smith, D.M., B.C. Larson, M.J. Kelty, and P. S. Ashton (1997). The practice of silviculture: applied forest ecology, ninth edition. John Wiley \& Sons, New York, $537 \mathrm{pp}$.

Smith, J.L., S.H. Zedaker, and R.C. Heer (1989). Estimating pine density and competition conditions in young pine plantations using $35 \mathrm{~mm}$ aerial photography. Southern Journal of Applied Forestry, 13: 107-112. 
Steger, C. (1998). "An unbiased detector of curvilinear structures". IEEE Transactions on Pattern Analysis and Machine Intelligence, 20: 537-559.

Stöckli, R., and P.L. Vidale (2004). European plant phenology and climate as seen in a 20-year AVHRR land-surface parameter dataset. International Journal of Remote Sensing, 25: 3303-3330.

St-Onge, B.A., and F. Cavayas (1995). Estimating forest stand structure from high resolution imagery using the directional variogram. International Journal of Remote Sensing, 16: 1999-2021.

Sullivan, T.P., A.S. Harestad, and B.M. Wikeem (1990). Section five, control of mammal damage, in Regenerating British Columbia's forests. Edited by D.P. Lavender, R. Parish, C.M. Johnson, G. Montgomery, A. Vyse, R.A. Willis, and D. Winston. UBS Press, Vancouver, pp. 302-318.

Taylor, N.W. (1999). Competing realities: the boreal forest at risk. Report of the subcommittee on boreal forest of the standing senate committee on agriculture and forestry. Ottawa, Ontario. Online paper: http://www.parl.gc.ca/36/1/parlbus/commbus/senate/com-e/bore-e/repe/rep09jun99-e.htm. Visited 11/11/01.

Ter-Mikaelian, M.T., R.G. Wagner, F.W. Bell, and C. Shropshire (1999). Comparison of photosynthetically active radiation and cover estimation for measuring the effects of interspecific competition on jack pine seedlings. Canadian Journal of Forest Research, 29: 883-889.

Tokeshi, M. (1997). Species coexistence and abundance: patterns and processes, in Biodiversity an Ecological Perspective. Edited by: T. Abe, S.A. Levin, and M. Higashi. Spinger-Verlag, New York, pp. 151-165.

Treitz, P.M., and P.J. Howarth (2000). Integrating spectral, spatial, and terrain variables for forest ecosystem classification. Photogrametric Engineering and Remote Sensing, 66: 305-317.

Tuominen, S. and A. Pekkarinen (2005). Performance of different spectral and textural aerial photograph features in multi-source forest inventory. Remote Sensing of Environment, 94: 256-268.

Wagner, R.G, F.W. Bell, and R.A Campbell. (2001). Chapter 6 stand tending and development, vegetation management, in Regenerating the Canadian forest: Principles and practices for Ontario. Edited. by R.G. Wagner and S.J. Colombo. Fitzhenry and Whiteside Ltd. Markham, Ontario, Canada, pp. 431-458.

Wagner, R.G., and M.S. McLaughlan (1996). Forest vegetation management alternatives: a forward to the guide. Northwest Science. \& Technology. Technical Note TN-38, 
in Vegetation management alternatives - guide to opportunities. Edited by F.W. Bell, M. McLaughlan, and J. Kerley. Ontario Ministry of Natural Resources, Thunder Bay, Ont. 8 pp.

Walstad, J.D., and P.J. Kuch (1987). Forest vegetation management for conifer production. John Wiley \& Sons, New York, $523 \mathrm{pp}$.

Walsworth, N.A., and D.J. King (1999). Image modeling of forest changes associated with acid mine drainage. Computers and Geosciences, 25: 567-580.

Wang, G.G., J.A. Siemens, V. Keenan, and D. Philippot (2000). Survival and growth of black and white spruce seedlings in relation to stock type, site preparation and plantation type in southeastern Manitoba. Forestry Chronicle, 76: 775-782.

Warner, T.A., J.Y. Lee, and J.B. McGraw (1999). Delineation and identification of individual trees in the eastern deciduous forest, in Proceedings international forum on automated interpretation of high spatial resolution digital imagery for forestry. Edited by D. A. Hill and D. G. Leckie. Victoria, B.C. Pacific Forestry Centre, Canadian Forest Service, Natural Resources Canada, Victoria, B.C. pp. 81-91.

Whitney, R.D., S. Greifenhagen, J. Mclaughlin, T. Meyer, and H.L. Gross (2001). Chapter 6 stand tending and protection, diseases and mycorrhizae, in Regenerating the Canadian forest. Edited by R.G. Wagner and S.J. Colombo. Fitzhenry and Whiteside Ltd. Markham, Ontario, Canada, pp. 459-482.

Willoughby, I. (1999). Future alternatives to the use of herbicides in British forestry. Canadian Journal of Forest Research, 29: 866-874.

Wulder, M.A., R.S. Skakum, W.A. Kurz, and J.C. White (2004). Estimating time since forest harvest using segmented Landsat ETM+ imagery. Remote Sensing of Environment, 93: 179-187.

Wulder, M., K.O. Niemann, and D.G. Goodenough (2000). Local maximum filtering for the extraction of tree locations and basal area from high spatial resolution imagery. Remote Sensing of Environment, 73: 103-114.

Wulder, M.A., S.E. Franklin, and M.B. Lavigne (1996). High resolution optical image texture for improved estimation of forest stand leaf area index. Canadian Journal of Remote Sensing, 22: 441-449.

Zhu, C., and X. Yang (1998). Study of remote sensing image texture analysis and classification using wavelet. International Journal of Remote Sensing, 19: 31973203. 